\title{
Spin Module Versions of Weyl's Reciprocity Theorem for Classical Kac-Moody Lie Algebras -An Application to Branching Rule Duality-
}

By

\author{
Koji HASEGAWA*
}

\begin{abstract}
We study tensor products of the spin modules (i.e. the Fermion Fock space representations) for classical (simple or affine) Kac-Moody Lie algebras. We find out that there are mutually commutant pairs of classical Kac-Moody algebras acting on the spin modules, and describe the irreducible decompositions in terms of Young diagrams. As applications, we obtain a simple explanation of Jimbo-Miwa's branching rule duality (i.e. isomorphisms between coset Virasoro modules) [JM], generalization thereof and the duality of the modular transformation rules of affine Lie algebra characters.
\end{abstract}

\section{Contents}

$\S 0$. Introduction

$\S 1$. Preliminaries for Classical Lie Algebras

1.1. Conventions

1.2. Affine Kac-Moody Lie algebras

1.3. Modules which admit actions of outer automorphisms

1.4. Notations for classical simple Lie algebras

1.5. Notations for classical affine Lie algebras

$\S 2$. Clifford Algebras and Spin Representations

2.1. Clifford algebra $C(W)$

2.2. The spin module $\bigwedge\left(W^{-}\right)$of $C(W)$

2.3. The spin module $\bigwedge\left(W_{N}^{-}\right)$of $\mathfrak{o}(N)$

2.4. The spin modules $\bigwedge\left(W_{N}^{\mathrm{Z}+h_{\mathrm{r}^{-}}}\right)$of $\mathfrak{o}(N)^{\wedge}$

$\S 3$. Dual Pairs of Classical Lie Algebras

3.1. Definition of representations

3.2. The irreducible decompositions: The main result in the finite dimensional case (Theorem 3.2)

3.3. Proof of Theorem 3.2

$\S 4$. Dual Pairs of Classical Affine Lie Algebras

4.1. Definition of representations

4.2. The irreducible decompositions: The main result in the affine Lie algebra case (Theorem 4.2)

4.3. Proof of Theorem 4.2: Case of $h=0$

4.4. Proof of Theorem 4.2 continued: Case of $h=1 / 2$

$\S 5$. An Application to the Duality of Branching Rules

5.1. Seesaw pairs and dualities

Communicated by M. Kashiwara, June 13, 1988. Revised February 23, 1989

* Mathematical Institute, Tôhoku University, Sendai 980, Japan 
5.2. Finite dimensional case

5.3. Affine case (I)

5.4. Affine case (II)

$\S 6$. An Application to the Duality of the Modular Transformation Rules of Characters

6.1. $S$-transformation rules of spin module characters

6.2. A duality of transformation rules of affine Lie algebra characters

$\S 7 . \quad$ Are There Affine Segal-Shale-Weil Module Versions?

§8. Appendix. The Fermion-Boson Correspondence

8.1. The Fermion-Boson correspondence

8.2. An explicit description of the automorphism $\sigma_{c y c}^{\#}$ References

\section{§0. Introduction}

\subsection{The purpose of the paper}

The Goddard-Kent-Olive construction, or the coset space representation, plays a significant role in realization of the irreducible unitary Virasoro modules in the discrete series $([\mathrm{GKO}])$. In connection with this method several authors ([GKO], [JM], [JIM1], [KW], [TK1], [Ya]) have noticed that there exists a kind of duality among the branching rules. Such duality interchanges the roles of the "rank parameter" and the "level parameter", as we will review in $\S 0.2$.

One of the purpose of this paper is to show that such duality is obtained as a reflection of "dual pairs on the spin modules"-mutually commutant pairs of (affine) Lie algebras which act on the Fermion Fock spaces.

In our main results, Theorems $\mathrm{AFF}$ and $\mathrm{FIN}$ in $\S 0.1$, we get the spin module versions of the dual pair $\left(\mathfrak{S}_{m}, G L(n, \mathbb{C})\right)$ on the module $\left(\mathbb{C}^{n}\right)^{\otimes m}$ (Weyl's reciprocity theorem [W]), where $\mathfrak{S}_{m}$ denotes the $m$-th symmetric group. They are dual pairs consisting of two affine (or finite dimensional) classical Lie algebras which act on the spin modules. Here spin modules are the $\mathfrak{D}(N)$-modules that correspond to the end points of the Dynkin diagrams and its affine analogues ([F1], [KP1]).

The fact that the spin modules admit various dual pairs has two applications.

Firstly we give a simple explanation of the duality among the branching rules through the method which is called "seesaw pair" by S. Kudla $([\mathrm{Ku}])$ in the finite dimensional theory $(\S 0.2(i), \S 5)$. For example the coset Virasoro modules associated to the two pairs

$$
\begin{aligned}
& \mathfrak{s p}(2 r)^{\wedge} \oplus \mathfrak{s p}(2 r)^{\wedge} \stackrel{\text { diagonal }}{\supset} \mathfrak{s l}(2 r)^{\wedge} \\
& \text { (level } l) \quad\left(\text { level } l^{\prime}\right) \quad\left(\text { level } l+l^{\prime}\right)
\end{aligned}
$$

and

$$
\begin{array}{crr}
\mathfrak{s p}\left(2 l+2 l^{\prime}\right)^{\wedge} & \supset \mathfrak{s p}(2 l)^{\wedge} \oplus \mathfrak{s p}\left(2 l^{\prime}\right)^{\wedge} \\
(\text { level } r) & (\text { level } r) & (\text { level } r)
\end{array}
$$

turn out to be mutually isomorphic, where $\mathfrak{g}^{\wedge}$ denotes the affinization of a simple Lie algebra $\mathfrak{g}$. In this case, the pair of dual pairs (both acting on the $\mathfrak{v}\left(4\left(l+l^{\prime}\right) r\right)^{\wedge}$-spin module) 


$$
\left\{\begin{array}{c}
\left(\mathfrak{s p}(2 r)^{\wedge} \oplus \mathfrak{s p}(2 r)^{\wedge}, \mathfrak{s p}(2 l)^{\wedge} \oplus \mathfrak{s p}\left(2 l^{\prime}\right)^{\wedge}\right) \\
\left(\mathfrak{s p}(2 r)^{\wedge}, \mathfrak{s p}\left(2 l+2 l^{\prime}\right)^{\wedge}\right)
\end{array}\right.
$$

is a seesaw pair. See Proposition $I$ in $\S 0.2$ and Tables $5.2 \sim 5.3$ for further examples.

The other application of dual pairs on the (affine) spin modules is the "duality" of modular transformation rules of affine Lie algebra characters. It is based on the fact that some of the characters for the spin modules are invariant under the $S$-transformation (§0.2(ii), §6).

While the spin representations are constructed on the exterior algebras, there is the symmetric algebra analogues, namely, so-called Segal-Shale-Weil modules or the oscillator representations ([FF]). There are also dual pairs on these modules in the finite dimensional case $([\mathrm{Ho}])$, but their affine correspondents are no longer dual pairs $(\S 0.4, \S 7)$.

In this introduction, we shall summarize the contents of this paper as follows.

$\S 0.1$ Main theorem

$\S 0.2$ Two applications of the main theorem

(i) Derivation of the branching rule duality

(ii) Derivation of the $S$-transformation rule duality

$\S 0.3$ How we obtained the main theorem

$\S 0.4$ Are there affine Segal-Shale-Weil versions?

$\S 0.5$ Notations and conventions in this paper

$\S 0.6$ Acknowledgement

\subsection{Main theorem}

For a finite dimensional reductive Lie algebra $\mathfrak{g}$ over the complex number field $\mathbf{C}$, let

$$
\mathfrak{g}^{\wedge}:=\mathfrak{g} \otimes_{\mathbf{C}} \mathbf{C}\left[t, t^{-1}\right] \oplus \mathbf{C} c
$$

be the corresponding affine Lie algebra $(\S 1.2 .1)$. Suppose $W=W_{N}$ is an $N$-dimensional vector space with a non-degenerate symmetric bilinear form $\{$,$\} , then put$

$$
W^{\mathbf{Z}+h}:=W_{N}^{\mathbf{Z}+h}:=W_{N} \otimes_{\mathbf{C}} t^{h} \mathbf{C}\left[t, t^{-1}\right]
$$

for $h=0$ or $1 / 2$ and define a bilinear form on $W^{\mathbf{Z}+h}$ by

$$
\left\{w \otimes t^{v}, w^{\prime} \otimes t^{v^{\prime}}\right\}:=\left\{w, w^{\prime}\right\} \delta_{v+v^{\prime}, 0} .
$$

Let $W^{\mathbf{Z}+h,-}$ be the maximal isotropic subspace with respect to this inner product which contains $W \otimes t^{-h} \mathbf{C}\left[t^{-1}\right]$, and let $\bigwedge\left(W^{\mathbf{Z}+h,-}\right)$ be the exterior algebra endowed with the spin module structure over $\mathfrak{o}(W)^{\wedge}([\mathrm{F} 1],[\mathrm{KP} 1] ; \S 2.4)$. In the physicists' terminology, $W^{\mathbf{Z}+h,-}$ is the space of Fermion creation operators in the quark model construction and the spin module $\bigwedge\left(W^{\mathbf{Z}+h,-}\right)$ for $h=0$ (resp. 1/2) is nothing but the Fermion Fock space of the Ramond sector (resp. the Neveu-Schwartz sector).

Then our main result is the answer to the following 
Problem 0.1. Let us define the Lie algebra pairs $\left(\mathfrak{g}_{\mathrm{L}}, \mathfrak{g}_{\mathrm{R}}\right)$ and their embeddings $\mathfrak{g}_{\mathrm{L}} \oplus \mathfrak{g}_{\mathrm{R}} \hookrightarrow \mathfrak{p}(W)$ as follows.

$$
\begin{aligned}
\mathfrak{g}_{\mathrm{L}} \oplus \mathfrak{g}_{\mathrm{R}}:= & \mathfrak{g l}\left(V_{1}\right) \oplus \mathfrak{s l}\left(V_{2}\right)\left[\hookrightarrow \operatorname{gl}\left(V_{1} \otimes V_{2}\right)\right] \hookrightarrow \mathfrak{o}(W), \\
& \text { where } W:=\left(V_{1} \otimes V_{2}\right) \oplus\left(V_{1} \otimes V_{2}\right)^{*} .
\end{aligned}
$$

$$
\begin{aligned}
& \mathfrak{g}_{\mathrm{L}} \oplus \mathfrak{g}_{\mathrm{R}}:=\mathfrak{s p}\left(V_{1}\right) \oplus \mathfrak{s p}\left(V_{2}\right) \hookrightarrow \mathfrak{p}(W), \quad W:=V_{1} \otimes V_{2} . \\
& \mathfrak{g}_{\mathrm{L}} \oplus \mathfrak{g}_{\mathrm{R}}:=\mathfrak{v}\left(V_{1}\right) \oplus \mathfrak{o}\left(V_{2}\right) \hookrightarrow \mathfrak{o}(W), \quad W:=V_{1} \otimes V_{2} .
\end{aligned}
$$

Then decompose the $\mathfrak{o}(W)^{\wedge}$-spin module $\bigwedge\left(W^{\mathbf{Z}+h,-}\right)(h=0,1 / 2)$ with respect to the subalgebra $\left(\mathfrak{g}_{\mathrm{L}} \oplus \mathfrak{g}_{\mathrm{R}}\right)^{\wedge} \hookrightarrow \mathfrak{o}(W)^{\wedge}$.

We need a definition.

Definition $0.1 \mathrm{I}\left(\mathrm{L}^{\Sigma \propto \mathfrak{g}^{\wedge}}(\lambda ; l)\right)$. Let $\Sigma$ be a group consisting of outer automorphisms of an affine Lie algebra $g^{\wedge}$ that preserve the canonical triangular decomposition $(\S 1.2 .1 .(4))$.

Then for an weight $\Lambda$ of $\mathfrak{g}^{\wedge}$, denoting the corresponding irreducible highest weight $\mathfrak{g}^{\wedge}$-module by $\mathbb{L}^{\mathfrak{g}^{\wedge}}(\Lambda)$, we put

$$
\mathbb{L}^{\Sigma \ltimes g^{\wedge}}(\Lambda):=\bigoplus_{\Lambda^{\prime} \in\{\sigma(\Lambda) ; \sigma \in \Sigma\}} \mathbb{L}^{g^{\wedge}}\left(\Lambda^{\prime}\right)
$$

$(\S \S 1.3 .2 \sim 4$.$) . Here \sigma(\cdot)$ is the adjoint action of $\sigma \in \Sigma$.

For a weight $\lambda$ of $\mathfrak{g}$ and $l \in \mathbb{C}$, we put

$$
\mathbb{L}^{\Sigma \ltimes \mathfrak{g}^{\wedge}}(\lambda ; l):=\mathbb{L}^{\Sigma \ltimes \mathfrak{g}^{\wedge}}\left(\lambda+l \hat{\Lambda}_{0}\right),
$$

where $\hat{\Lambda}_{0}$ denotes the 0 -th fundamental weight of $\mathfrak{g}^{\wedge}(\S 1.2 .3(1))$.

Now the statement is as follows.

Theorem AFF (Theorem 4.2.). As a $\mathfrak{g}_{\mathbf{L}}^{\wedge} \oplus \mathfrak{g}_{\hat{\mathrm{R}}}^{\hat{}}$-module ((AA), (CC) or (OO)), the $\mathfrak{o}(W)^{\wedge}$-spin module $\bigwedge\left(W^{\mathbb{Z}+h,-}\right)(h=0,1 / 2)$ decomposes as follows.

\begin{tabular}{|c|c|c|}
\hline $\mathfrak{g}_{\mathrm{L}}^{\wedge}, \mathfrak{g}_{\hat{R}}^{\wedge}$ & $\mathfrak{g l}(l)^{\wedge}, \mathfrak{s l}(r)^{\wedge}$ & $\mathfrak{s p}(2 l)^{\wedge}, \mathfrak{s p}(2 r)$ \\
\hline$\Sigma_{\hat{L}}, \Sigma_{\hat{R}}$ & $\left\langle\sigma_{c y c}^{\#}\right\rangle,\{i d\}$ & $\{i d\},\{i d\}$ \\
\hline$R, L$ & $r, l$ & $r, l$ \\
\hline$N=\operatorname{dim} W$ & $2 l r$ & $4 l r$ \\
\hline$\hat{\lambda}_{\mathrm{L}}(Y), \hat{\lambda}_{\mathrm{R}}(Y)\left\{\begin{array}{l}h=0 \\
h=\frac{1}{2}\end{array}\right.$ & $\left\{\begin{array}{l}(Y,[Y]-l r / 2),{ }^{t} Y \\
(Y,[Y]),{ }^{t} Y\end{array}\right.$ & $\left\{\begin{array}{l}Y, Y^{\dagger} \\
Y,{ }^{t} Y\end{array}\right.$ \\
\hline$Y$ runs over & $Y \in \mathscr{Y}_{l, r-1}$ & $Y \in \mathscr{Y}_{l, r}$ \\
\hline
\end{tabular}

$$
\bigwedge\left(W^{\mathbb{Z}+h,-}\right) \simeq \bigoplus_{Y} \mathbb{L}^{\Sigma_{\mathrm{L}}^{\hat{L}} \times \mathrm{g}_{\mathrm{L}}}\left(\hat{\lambda}_{\mathrm{L}}(Y) ; R\right) \otimes \mathbb{L}^{\Sigma_{\mathrm{R}}^{\hat{R}} \times \mathfrak{g}_{\mathrm{R}}}\left(\hat{\lambda}_{\mathrm{R}}(Y) ; L\right)
$$

See Table AFF for $\Sigma_{\mathrm{L}}^{\wedge}, \Sigma_{\mathrm{R}} ; \hat{\lambda}_{\mathrm{L}}, \hat{\lambda}_{\mathrm{R}} ; R, L$ and where $Y$ varies.

List 4.2 gives the complete list of the highest weight vectors with respect to $\mathfrak{g}_{\hat{L}} \oplus \mathfrak{g}_{\mathbf{R}}$.

\section{Table AFF}

List of HWV's

List 4.2.ÂÂA

List 4.2. $\hat{\mathrm{C}} \hat{\mathrm{C}}$ 
Table AFF Continued

\begin{tabular}{|c|c|c|c|}
\hline $\mathfrak{g}_{\mathbf{L}}, \mathfrak{g}_{\hat{R}}$ & $\mathfrak{o}(2 l+1)^{\wedge}, \mathfrak{o}(2 r+1)^{\wedge}$ & $\mathfrak{o}(2 l)^{\wedge}, \mathfrak{o}(2 r+1)^{\wedge}$ & $\mathfrak{o}(2 l)^{\wedge}, \mathfrak{o}(2 r)^{\wedge}$ \\
\hline$\Sigma_{\mathbf{L}}^{\wedge}, \Sigma_{\mathbf{R}}$ & $\left\langle\sigma_{01}\right\rangle,\left\langle\sigma_{01}\right\rangle$ & $\left\langle\sigma_{01}, \sigma_{l-1, l}\right\rangle,\left\langle\sigma_{01}\right\rangle$ & $\left\langle\sigma_{01}, \sigma_{l-1, l}\right\rangle,\left\langle\sigma_{01}, \sigma_{r-1, r}\right\rangle$ \\
\hline$R, L$ & $2 r+1,2 l+1$ & $2 r+1,2 l$ & $2 r, 2 l$ \\
\hline$N=\operatorname{dim} W$ & $(2 l+1)(2 r+1)$ & $2 l(2 r+1)$ & $4 l r$ \\
\hline$\hat{\lambda}_{\mathbf{L}}(Y), \hat{\lambda}_{\mathbf{R}}(Y)\left\{\begin{array}{l}h=0 \\
h=\frac{1}{2}\end{array}\right.$ & $\left\{\begin{array}{l}Y+\Lambda_{l}, Y^{\dagger}+\Lambda_{r} \\
Y,{ }^{t} Y\end{array}\right.$ & $\left\{\begin{array}{l}Y+\Lambda_{l}, Y^{\dagger} \\
Y,{ }^{t} Y\end{array}\right.$ & $\left\{\begin{array}{l}Y, Y^{\dagger} \\
Y,{ }^{t} Y\end{array}\right.$ \\
\hline$Y$ runs over & $Y \in \mathscr{Y}_{l, r}$ & $Y \in \mathscr{Y}_{l, r}$ & $Y \in \mathscr{Y}_{l, r}$ \\
\hline List of HWV's & List 4.2. $\hat{\mathrm{B}} \hat{\mathrm{B}}$ & List 4.2. $\hat{D} \hat{B}$ & List 4.2. $\hat{D} \hat{D}$ \\
\hline
\end{tabular}

\section{Notations for Table AFF}

(i) $\bullet \sigma_{i, j}$ : the automorphism induced by the symmetry $i \leftrightarrow j$ of the Dynkin diagram $(\S 1.5)$,

- $\sigma_{c y c}^{\#}:$ an infinite order automorphism of $\mathfrak{g l}(l)^{\wedge}$ whose restriction to $\mathfrak{s l}(l)^{\wedge} \simeq$ $\mathfrak{g}\left(A_{l-1}^{(1)}\right)$ is the order $l$ cyclic diagram automorphism $\sigma_{c y c}(\S 1.3 .4, \S 1.5 .2)$.

(ii) - $\mathscr{Y}_{l, r}$ : the set of Young diagrams contained in the $l \times r$-rectangle,

- ${ }^{t} Y \in \mathscr{Y}_{r, l}, Y^{\dagger} \in \mathscr{Y}_{r, l},[Y] \in \mathbb{Z}_{\geq 0}$ for $Y \in \mathscr{Y}_{l, r}$ : respectively denotes the transposed, the complement, and the size of $Y$ (see $\S 1.4 .1$ ).

(iii) - A Young diagram is identified with a weight as usual. (see (3.3) of $\S \S 1.4 .2 \sim 5)$.

- $\Lambda_{l}$ : the $l$-th fundamental weight for $\mathfrak{o}(2 l)$ or $\mathfrak{o}(2 l+1)$ (not the fundamental weight $\hat{\Lambda}_{l}$ for $\mathfrak{D}(2 l)^{\wedge}$ or $\mathfrak{D}(2 l+1)^{\wedge}$, but its classical part).

- For the case of affine Lie algebra $\operatorname{gl}(l)^{\wedge},(\lambda, a)$ denotes the weight for $\operatorname{gl}(l)$ whose restriction to $\mathfrak{s l}(l)$ coinsides with $\lambda$ and $a=\left\langle(\lambda, a), \mathbb{1}_{l}\right\rangle \in \mathbb{C}$, where $\mathbb{1}_{l} \in \mathfrak{g l}(l)$ denotes the identity matrix.

The finite dimensional version of this theorem is also obtained. For a group $\Sigma$ consisting of diagram automorphisms and for a weight $\lambda$, denoting by $\mathbb{L}^{g}(\lambda)$ the irreducible highest weight $\mathrm{g}$-module, we put

$$
\mathbb{L}^{\Sigma \times \mathrm{g}}(\lambda):=\bigoplus_{\lambda^{\prime} \in\{\sigma(\lambda) ; \sigma \in \Sigma\}} \mathbf{L}^{\mathrm{g}}\left(\lambda^{\prime}\right) .
$$

We also employ the notation specified in the above. Then we have

Theorem FIN (Theorem 3.2.). As a $\mathrm{g}_{\mathrm{L}} \oplus \mathrm{g}_{\mathrm{R}}$-module ((AA), (CC) or (OO)), the $\mathfrak{o}(W)$-spin module $\bigwedge\left(\mathbb{C}^{[N / 2]}\right)(N:=\operatorname{dim} W ; \S 2.3)$ decomposes as follows (see Table FIN for $\left.\Sigma_{\mathrm{L}}, \Sigma_{\mathrm{R}} ; \lambda_{\mathrm{L}}, \lambda_{\mathrm{R}}\right)$

$$
\bigwedge\left(\mathbf{C}^{[N / 2]}\right) \simeq \bigoplus_{Y \in \mathscr{Y}_{l, r}} \mathbb{L}^{\Sigma_{L} \times \mathrm{g}_{\mathrm{L}}}\left(\lambda_{\mathrm{L}}(Y)\right) \otimes \mathbb{L}^{\Sigma_{\mathrm{R}} \times \mathrm{g}_{\mathrm{R}}}\left(\lambda_{\mathrm{R}}(Y)\right)
$$

List $3.2(A A-D D)$ gives the complete list of the highest weight vector of each irreducible component with respect to $\mathrm{g}_{\mathrm{L}} \oplus \mathrm{g}_{\mathrm{R}}$.

For a given classical simple Lie algebra $\mathfrak{g}_{\mathfrak{R}}$, all its irreducible modules appear in the above decomposition (8) as we vary the counterpart $\mathrm{g}_{\mathrm{L}}$. 


\section{Table FIN}

$\begin{array}{ccc}\mathrm{g}_{\mathrm{L}}, \mathrm{g}_{\mathrm{R}} & \mathfrak{g l}(l), \mathfrak{s l}(r) & \mathfrak{s p}(2 l), \mathfrak{s p}(2 r) \\ \Sigma_{\mathrm{L}}, \Sigma_{\mathrm{R}} & \{i d\},\{i d\} & \{i d\},\{i d\} \\ N & 2 l r & 4 l r \\ \lambda_{\mathrm{L}}(Y), \lambda_{\mathrm{R}}(Y) & (Y,[Y]-l r / 2),{ }^{t} Y & Y, Y^{\dagger}\end{array}$

List of HWV's

List 3.2.AA

List 3.2.CC

Table FIN Continued

$\begin{array}{cccc}\mathfrak{g}_{\mathrm{L}}, \mathrm{g}_{\mathrm{R}} & \mathfrak{o}(2 l+1), \mathfrak{o}(2 r+1) & \mathfrak{o}(2 l), \mathfrak{o}(2 r+1) & \mathfrak{o}(2 l), \mathfrak{o}(2 r) \\ \Sigma_{\mathrm{L}}, \Sigma_{\mathrm{R}} & \{i d\},\{i d\} & \left\langle\sigma_{l-1, l}\right\rangle,\{i d\} & \left\langle\sigma_{l-1, l}\right\rangle,\left\langle\sigma_{r-1, r}\right\rangle \\ N & (2 l+1)(2 r+1) & 2 l(2 r+1) & 4 l r \\ \lambda_{\mathrm{L}}(Y), \lambda_{\mathrm{R}}(Y) & Y+\Lambda_{l}, Y^{\dagger}+\Lambda_{r} & Y+\Lambda_{l}, Y^{\dagger} & Y, Y^{\dagger} \\ \text { List of HWV's } & \text { List } 32 \mathrm{BB} & \text { List 32 } & \end{array}$

List of HWV's

List 3.2.BB

List 3.2.DB

List 3.2.DD

These results can be summarized by using the notion of "dual pair" due to $\mathbb{R}$. Howe, and it turns out to be useful in explaining the branching rule dualities to do so. Let us recall

Definition 0.1b (Dual Pair [Ho]). For an associative algebra $A$, let us denote by $\operatorname{Irr}(A)$ the isomorphism classes of irreducible $A$-modules and by $\mathbb{L}_{\lambda}^{A}$ a representative for $\lambda \in \operatorname{Irr}(A)$.

For two algebras $A$ and $A^{\prime}$, which act on the same vector space $V$ with satisfying $\left[A, A^{\prime}\right]=0$, we say that the pair $\left(A, A^{\prime}\right)$ is a dual pair on $V$ if the following condition (9) is satisfied.

(9) There exist some index set $I$ and injections

$$
\lambda: I \rightarrow \operatorname{Irr}(A), \quad \lambda^{\prime}: I \rightarrow \operatorname{Irr}\left(A^{\prime}\right)
$$

such that $V$ is decomposed as follows.

$$
V \simeq \bigoplus_{l \in I} \mathbb{L}_{\lambda(l)}^{A} \otimes \mathbb{L}_{\lambda^{\prime}(l)}^{A^{\prime}}
$$

(that is, $\lambda(l)$ determines $\lambda^{\prime}(l)$ and vice versa).

A $g$-module $\mathbb{L}^{\Sigma \ltimes g}(\lambda)$ appearing in Theorems $A F F$ and $F I N$ gives rise to an irreducible module of the algebra $\mathrm{C}[\Sigma] \ltimes U(\mathrm{~g})$, which is defined by the natural action of $\Sigma$ on the enveloping algebra $U(\mathfrak{g})$ (§1.3.2). (Also note that $\mathbb{L}^{\{i d\} \times g}(\lambda)=\mathbb{L}^{\mathfrak{g}}(\lambda)$.) Therefore Theorems read as

Corollary to Theorems AFF and FIN.

(i) In each case of Theorem $A F F$, the pair

$$
\left(\mathbb{C}\left[\Sigma_{\mathbf{L}}^{\wedge}\right] \ltimes U\left(\mathfrak{g}_{\mathbf{L}}^{\wedge}\right), \mathbb{C}\left[\Sigma_{\mathbf{R}}^{\wedge}\right] \ltimes U\left(\mathfrak{g}_{\mathbf{R}}^{\wedge}\right)\right)
$$

of algebras forms a dual pair on $\bigwedge\left(W_{N}^{\mathbb{Z}+h,-}\right)$. 
(ii) [Ho1] In each case of Theorem FIN, the pair

$$
\left(\mathbb{C}\left[\Sigma_{L}\right] \ltimes U\left(g_{L}\right), \mathbb{C}\left[\Sigma_{R}\right] \ltimes U\left(g_{R}\right)\right)
$$

forms a dual pair on $\bigwedge\left(\mathbb{C}^{[N / 2]}\right)$.

\subsection{Two applications of the main theorem}

\section{(i) Derivation of the branching rule duality}

Here we wish to show in a typical example how Theorem AFF works in explaining the branching rule dualities. We need a definition.

Definition 0.2 (Branching module). Suppose that $A_{1}$ is an algebra and $A_{2}$ its subalgebra, then put

$$
\mathbb{B}\left(A_{1} \supset A_{2}\right)_{\lambda}^{\mu}:=\operatorname{Hom}_{A_{2}}\left(\mathbb{L}_{\mu}^{A_{2}}, \mathbb{L}_{\lambda}^{A_{1}}\right)
$$

for $\lambda \in \operatorname{Irr} A_{1}$ and $\mu \in \operatorname{Irr} A_{2}$.

Assuming the complete reducibility, we have an isomorphism

$$
\mathbb{L}_{\lambda}^{A_{1}} \simeq \bigoplus_{\mu} \mathbf{B}\left(A_{1} \supset A_{2}\right)_{\lambda}^{\mu} \otimes \mathbb{L}^{A_{2}}
$$

as an $A_{2}$-module, and we call $\mathbf{B}\left(A_{1} \supset A_{2}\right)_{\lambda}^{\mu}$ the branching module associated to the inclusion (or restriction) $A_{1} \supset A_{2}$.

(In other words, branching module is the space of "highest weight vectors" in $\mathbf{L}^{A_{1}}$ with respect to $A_{2}$ whose weight is $\mu$ ).

Recall a branching rule duality

Example 0.2 (A branching rule duality [GKO] [JM] [KW] [TK1, 2] [Y]). There is an isomorphism between the coset Virasoro modules

$$
\begin{gathered}
\mathbb{B}\left(\mathfrak{s l}(2)^{\wedge} \oplus \mathfrak{s l}(2)^{\wedge} \stackrel{\text { diagonal }}{\supset} \mathfrak{s l}(2)^{\wedge}\right)_{(l-1-p) \hat{\Lambda}_{0}+p \hat{\Lambda}_{1}, \hat{\Lambda}_{r}}^{(l-q) \hat{\Lambda}_{0}+q \hat{A}_{1}} \\
\simeq \mathbb{B}\left(\mathfrak{s p}(2 l)^{\wedge} \supset \mathfrak{s p}(2 l-2)^{\wedge} \oplus \mathfrak{s p}(2)^{\wedge}\right)_{\hat{\Lambda}_{q}}^{\hat{A}_{p}, \hat{\Lambda}_{r}} \\
(l=2,3, \ldots ; 0 \leq p \leq l-1,0 \leq q \leq l, 0 \leq r \leq 1),
\end{gathered}
$$

where $\hat{\Lambda}_{j}$ denotes the $j$-th (affine) fundamental weight.

Now we can deduce this duality from our viewpoint, by realizing these branching modules in the spin module with the help of Theorem AFF.

In fact, consider the pair of Lie algebra pairs

$$
\left\{\begin{array}{l}
\left(\mathfrak{g}_{1}, \mathfrak{g}_{1}^{\prime}\right):=\left(\mathfrak{s l}(2)^{\wedge} \oplus \mathfrak{s l}(2)^{\wedge}, \mathfrak{s p}(2 l-2)^{\wedge} \oplus \mathfrak{s p}(2)^{\wedge}\right) \\
\left(\mathfrak{g}_{2}, \mathfrak{g}_{2}^{\prime}\right):=\left(\mathfrak{s l}(2)^{\wedge}, \mathfrak{s p}(2 l)^{\wedge}\right)
\end{array}\right.
$$

acting on the $\mathfrak{v}(4 l)^{\wedge}$-spin module $\bigwedge\left(W_{4 l}^{\mathbf{Z}+h,-}\right) \simeq \bigwedge\left(W_{4 l^{-4}}^{\mathbf{Z}+{ }^{-}}\right) \otimes \wedge\left(W_{4}^{\mathbf{Z}+h,-}\right)$ through the embedding 0.1(2). They satisfy the following inclusion relations (the seesaw pair property $[\mathrm{Ku}])$ in End $\bigwedge\left(W_{4 l}^{\mathbf{Z}+h,-}\right)$. 


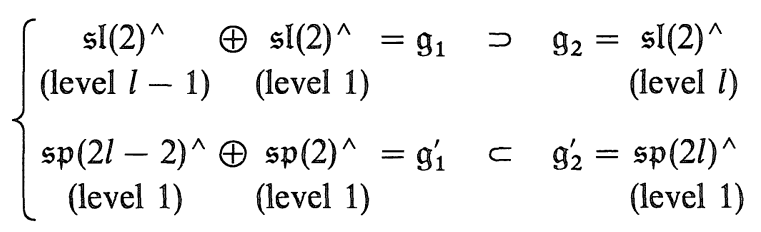

On the other hand, due to Theorem AFF for $\left(\mathfrak{g}_{L}^{\wedge}, \mathfrak{g}_{R}^{\wedge}\right)=\left(\mathfrak{s p} \mathfrak{p}^{\wedge}, \mathfrak{s p}^{\wedge}\right)$, the pairs $\left(U\left(\mathrm{~g}_{i}\right), U\left(\mathrm{~g}_{i}^{\prime}\right)\right)(i=1,2)$ form dual pairs, that is, roughly speaking, the module $\bigwedge\left(W_{4 l}^{\mathbb{Z}+h,-}\right)$ is decomposed as follows.

$$
\left\{\begin{aligned}
\bigwedge\left(W_{4 l}^{\mathbb{Z}+h,-}\right) & \simeq \bigoplus_{\lambda \in I_{1} \subset \operatorname{Irr} g_{1}} L^{g_{1}}{ }_{\lambda} \otimes L^{g_{1}^{\prime}}{ }_{\lambda^{\prime}} \\
& \simeq \bigoplus_{\mu \in I_{2} \subset \operatorname{Irr} g_{2}} L^{g_{2}}{ }_{\mu} \otimes \mathbb{L}^{g_{2}^{\prime}}{ }_{\mu^{\prime}}
\end{aligned}\right.
$$

Let us decompose $\bigwedge\left(W_{4 l}^{\mathbb{Z}+h,-}\right)$ as a $\mathfrak{g}_{1}^{\prime} \oplus \mathrm{g}_{2}$-module in two ways:

$$
\left\{\begin{aligned}
\bigwedge\left(W_{4 l}^{\mathbb{Z}+h,-}\right) & \simeq \bigoplus_{\lambda}\left(\bigoplus_{\mu} \mathbb{B}\left(\mathfrak{g}_{1} \supset \mathfrak{g}_{2}\right)_{\lambda}^{\mu} \otimes \mathbb{L}^{\mathfrak{g}_{2}}\right) \otimes \mathbb{L}^{g^{\prime}}{ }_{\lambda^{\prime}} \\
& \simeq \bigoplus_{\mu} \mathbb{L}^{\mathfrak{g}_{2}}{ }_{\mu} \otimes\left(\bigoplus_{\lambda^{\prime}} \mathbb{B}\left(\mathfrak{g}_{2}^{\prime} \supset \mathfrak{g}_{1}^{\prime}\right)_{\mu^{\prime}}^{\lambda^{\prime}} \otimes \mathbb{L}^{\mathfrak{g}^{\prime}}{ }_{\lambda^{\prime}}\right),
\end{aligned}\right.
$$

then we find an isomorphism $\mathbb{B}\left(\mathfrak{g}_{1} \supset \mathfrak{g}_{2}\right)_{\lambda}^{\mu} \simeq \mathbb{B}\left(\mathfrak{g}_{2}^{\prime} \supset \mathfrak{g}_{1}^{\prime}\right)_{\mu^{\prime}}^{\lambda^{\prime}}$. Writing down $\lambda$ 's and $\mu$ 's explicitly, this gives Example 0.2 .

From our viewpoint in the above, it is straightforward to generalize this duality (3) for the pair of 0.0.(1, 2).

Generally speaking, one seesaw pair, that is, a pair of dual pairs that satisfy the property (5) (\$5.1), provides one duality of branching rules (Proposition 5.1). Theorems $\mathrm{AFF}$ and FIN yields various seesaw pairs and hence the corresponding dualities $(\S \S 5.2 \sim 5.4)$ : it turns out that the branching rule duality already exists in the finite dimensional theory. In particular, intertwining the homogeneous picture representations and the principal picture representations ([F2]) we deduce

Proposition 耳. (Another type of duality, Cor. 5.4.2). There is a "duality" between the restrictions associated to the inclusion

$$
\begin{aligned}
\mathbb{C}\left[\left\langle\sigma_{c y c}^{\#}\right\rangle\right] \ltimes U\left(\mathfrak{g l}(l) \otimes \mathbb{C}\left[t, t^{-1}\right] \oplus \mathbb{C} c\right) \\
\stackrel{\leftrightarrow}{\hookleftarrow} \mathbb{C}\left[\left\langle\sigma_{c y c}^{\#}\right\rangle\right] \ltimes U\left(\mathfrak{g l}(l) \otimes \mathbb{C}\left[t^{m}, t^{-m}\right] \oplus \mathbb{C} c\right)
\end{aligned}
$$

(See "Notation for Table AFF" for $\sigma_{c y c}^{\#}$ ) and that to the invariant subalgebra with respect to the cyclic diagram automorphism

$$
\mathfrak{s l}(m r)^{\wedge} \stackrel{i^{\prime}}{\leftrightarrow}\left(\mathfrak{s l}(m r)^{\wedge}\right)^{\left\langle\sigma_{c y c}{ }^{r}\right\rangle} \quad\left[\simeq \mathfrak{s l}(r)^{\wedge}\right. \text {, Prop. 5.4.2], }
$$

in the sense that they yield mutually isomorphic branching modules: for any $y \in \mathscr{Y}_{l, r-1}$ and $Y \in \mathscr{Y}_{l, m r-1}$ we have

$$
\mathbb{B}(i)_{(y,[y] ; r)}^{(Y,[Y] ; m r)} \simeq \mathbb{B}\left(i^{\prime}\right)_{(t Y ; l)}^{(t y ; l)}
$$

as the coset $\mathscr{V}_{i r \text {-modules. }}$

\section{(ii) Derivation of the S-transformation rule duality}

As a next application of Theorem AFF, we shall deduce a "duality" concerning with the modular transformation rules of characters. It is based on the fact that the 
character of the Fermion Fock space ch $\bigwedge\left(W_{N}^{\mathbf{Z}+1 / 2,-}\right)$, which is essentially an $N$-fold product of

$$
q^{-1 / 48} \prod_{n=1}^{\infty}\left(1+q^{n+1 / 2}\right), \quad q=e^{2 \pi i \tau},
$$

is invariant under the "S-transformation" on the upper half plane

$$
\mathbb{S}: \tau \mapsto-1 / \tau \text {. }
$$

For example, let us apply this transformation to the following "character bilinear identity" which is equivalent to Theorem AFF for $\left(\mathfrak{g}_{L}^{\wedge}, \mathfrak{g}_{R}^{\wedge}\right)=\left(\mathfrak{s p}(2 l)^{\wedge}, \mathfrak{s p}(2 r)^{\wedge}\right), h=1 / 2$ :

$$
\operatorname{ch} \bigwedge\left(W_{4 l r}^{\mathbb{Z}+1 / 2,-}\right)=\Sigma_{Y \in \mathscr{Y}_{l, r}} \chi_{Y, r}^{\mathfrak{S p}(2 l)^{\wedge}} \cdot \chi_{t Y, l}^{\mathfrak{S P}(2 r)^{\wedge}},
$$

where $\chi_{Y, r}^{\mathfrak{S p}(2 l)^{\wedge}}$ denotes the normalized character $(\$ 1.2 .3)$ of the module $\mathbb{L}^{\mathfrak{s p}(2 l)^{\wedge}}(Y ; r)$. Then the above mentioned invariance of the spin module character $\operatorname{ch} \bigwedge\left(W_{4 l r}^{\mathbb{Z}+1 / 2,-}\right)$ yields the following "duality" of the S-transformation rules:

Proposition $\mathbf{H}$ (A duality of transformation rules, Cor. 6.2a). Let $\left(M_{y, y^{\prime}}^{(l, r)}\right)_{y, y^{\prime} \in \mathscr{Y}_{l, r}} b e$ the scalar matrix of the $\mathbf{S}$-transformation rule for level $r$ characters of $\mathfrak{s p}(2 l)^{\wedge}$ :

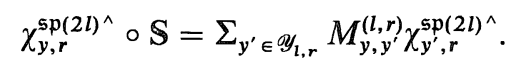

Then there is a relation $M_{y, y^{\prime}}^{(l, r)}=\overline{M_{t y, y^{\prime}}^{(r, l)}}$ for $y, y^{\prime} \in \mathscr{Y}_{l, r}$, where the bar denotes the complex conjugation and ${ }^{t} Y \in \mathscr{Y}_{r, l}$ denotes the transposed diagram for a Young diagram $Y \in \mathscr{Y}_{l, r}$.

\subsection{How we obtained the main theorem}

Let us sketch the way how we find the dual pairs. Our motivation is to get a better understanding of the Jimbo-Miwa's branching rule duality. Keeping in mind that the Weyl's dual pair $\left(\mathfrak{S}_{m}, G L(n)\right)$ on $\left(\mathbb{C}^{n}\right)^{\otimes m}$ provides close relation between the $\Im_{m}$-modules and $G L(n)$-modules ([R, Chap. 3]), which can be recognized as a duality in the sence of Jimbo-Miwa, we posed the following

Problem 0.3. Describe the commutant algebra of the $\mathfrak{o}(L)^{\wedge}$-action on the tensor product $\bigwedge\left(W_{L}^{\mathbf{Z}+h,-}\right)^{\otimes R}$ of the spin module $\bigwedge\left(W_{L}^{\mathbf{Z}+h,-}\right)$ and decompose it into irreducible components.

In our consideration, important is the following isomorphisms of vector spaces (in $h=1 / 2$ case).

$$
\bigwedge\left(W_{L}^{\mathbf{Z}+h,-}\right)^{\otimes R} \simeq \bigwedge\left(\oplus^{R} W_{L}^{\mathbf{Z}+h,-}\right) \simeq \bigwedge\left(W_{L R}^{\mathbf{Z}+h,-}\right)
$$

In other words, the module $\bigwedge\left(W_{L}^{\mathbb{Z}+h,-}\right)^{\otimes R}$ has the $\mathfrak{o}(L R)^{\wedge}$-spin module structure and hence we can introduce an $\mathfrak{v}(R)^{\wedge}$-action on it. We hope that $\mathfrak{o}(R)^{\wedge}$ generates the commutant algebra of the $\mathfrak{D}(L)^{\wedge}$-action. Is it right? Thus we are lead to Problem 0.1 for the case (OO) itself.

For the algebras $\mathfrak{g}_{\mathrm{L}} \oplus \mathfrak{g}_{\mathrm{R}} \subset \mathfrak{v}(W)$ in Problem 0.1, first we can show (Propositions 1.2 and 1.4) that any irreducible $\mathrm{g}_{\mathrm{L}} \oplus \mathrm{g}_{\mathbf{R}}$-modules appear with finite multiplicities in the $\mathfrak{o}(W)^{\wedge}$-spin module $\bigwedge\left(W^{\mathbb{Z}+h,-}\right)$. This is due to the central charge identity 


$$
\mathbb{Z}^{\mathfrak{o}(W)^{\wedge}}=Z^{\mathfrak{g}_{\mathbf{L}}^{\wedge}}+Z^{\mathfrak{g}_{\mathrm{R}}^{\wedge}}
$$

where $Z^{\mathfrak{g}^{\wedge}}:=\frac{c \operatorname{dim} \mathfrak{g}}{c+g}$ is the Virasoro central charge for the Segal-Sugawara operators associated with $\mathfrak{g}^{\wedge}(1.2 .2(3)$ ), denoting by $c$ (resp. $g$ ) the level (resp. the dual Coxeter number) of $\mathfrak{g}^{\wedge}$. For example, (2) turns to the identity

$$
\frac{L \cdot R}{2}=\frac{R \cdot L(L-1) / 2}{R+(L-2)}+\frac{L \cdot R(R-1) / 2}{L+(R-2)}
$$

for the pair $\left(\mathfrak{g}_{\mathrm{L}}, \mathfrak{g}_{\mathbb{R}}^{\wedge}\right)=\left(\mathfrak{o}(\mathbb{L})^{\wedge}, \mathfrak{o}(\mathbb{R})^{\wedge}\right)$. Here we must note that [GKO] first recognized the importance of computation of central charges.

To get accurate decomposition of the spin modules, we must compute their characters as $\left(\mathfrak{g}_{\mathrm{L}} \oplus \mathfrak{g}_{\mathrm{R}}\right)^{\wedge}$-modules and obtain "character bilinear identity" like $0.2(13)$. We get explicit form of the character identities due to the Weyl-Kac formula and its application "complementary decomposition formula" of Jimbo-Miwa [JM]. Thus we obtain exact forms of dual pairs as in Theorem AFF.

After obtained Theorem AFF, we obtained Theorem FIN as its finite dimensional version. Indeed, in terms of character identities we can obtain Theorem FIN as the "classical limit" $q \rightarrow 0$ of Theorem AFF for $h=0$ (however in this paper we derive Theorem $\mathbb{F I N}$ independently to Theorem $\mathrm{AFF}$, for the clearness of discussions).

\subsection{Are there affine Segal-Shale-Weil versions?}

Thus we have succeeded in explaining the dualities, by constructing and using the dual pairs of affine Lie algebras on the spin modules. Our construction of the dual pairs was quite similar to that of dual pairs on the Segal-Shale-Weil modules (or the oscilator representation) over the finite dimensional algebra $\mathfrak{s p}(W)$, which is treated by Howe's paper [Ho] and references therein. It is natural to seek the possibility of the affine Segal-Shale-Weil analogues.

But the central charge calculation shows that the answer is negative.

Proposition III (There are no affine Segal-Shale-Weil versions, Proposition 7.1). Let $\mathscr{S}$ be the affine Segal-Shale-Weil module over $\mathfrak{s p}(W)^{\wedge}[\mathbb{F F}]$, and consider $\mathscr{S}$ as a module over the algebra

$$
\mathfrak{s p}\left(V_{1}\right)^{\wedge} \oplus \mathfrak{o}\left(V_{2}\right)^{\wedge}
$$

(resp. $\left.\mathfrak{g l}\left(V_{1}\right)^{\wedge} \oplus \mathfrak{s l}\left(\mathbb{V}_{2}\right)^{\wedge}\right)$ according to the inclusion

$$
\mathfrak{s p}\left(V_{1}\right) \oplus \mathfrak{v}\left(V_{2}\right) \subset \mathfrak{s p}(W), \quad W:=\mathbb{V}_{1} \otimes V_{2}
$$

(resp. $\left.\mathfrak{g l}\left(V_{1}\right) \oplus \mathfrak{s l}\left(V_{2}\right)\left[\subset \mathfrak{g l}\left(V_{1} \otimes V_{2}\right)\right] \subset \mathfrak{s p}(W), \quad W:=\left(V_{1} \otimes V_{2}\right) \oplus\left(V_{1} \otimes V_{2}\right)^{*}\right) . \quad$ Moreover assume that

$$
\operatorname{dim} V_{1}+2 \neq 2 \operatorname{dim} \mathbb{V}_{2} \quad \text { and } \quad 2 \operatorname{dim} V_{1} \neq \operatorname{dim} \mathbb{V}_{2}-2
$$

(resp. $2 \operatorname{dim} V_{1} \neq \operatorname{dim} V_{2}$ and $\left.\operatorname{dim} V_{1} \neq 2 \operatorname{dim} V_{2}\right)$. Then the pair $\left(\mathfrak{s p}\left(V_{1}\right)^{\wedge}, \mathfrak{o}\left(V_{2}\right)^{\wedge}\right)($ resp. $\left.\left(\mathfrak{g l}\left(V_{1}\right)^{\wedge}, \mathfrak{s l}\left(V_{2}\right)^{\wedge}\right)\right)$ does not form a dual pair on $\mathscr{S}$. 


\subsection{Notations and conventions in this paper}

Throughout this paper, we fix the conventions on classical Lie algebras (such as the choice of Chevalley generators, parametrization of weights via Young diagrams, etc) as in Sections 1.4 and 1.5.

The Virasoro algebra $\mathscr{V}_{i}$ is usually defined by

$$
\begin{gathered}
\mathscr{V}_{i \imath}:=\bigoplus_{m \in \mathbf{Z}} \mathbf{C l}(m) \oplus \mathbf{C} z, \\
{[l(m), l(n)]:=(m-n) l(m+n)+\frac{m^{3}-m}{12} \delta_{m+n, 0} z, \quad\left[\mathscr{V}_{i r}, z\right]:=0 .}
\end{gathered}
$$

In this paper, we will use the following basis of $\mathscr{V}_{i} r$ :

$$
[d(m), d(n)]=(m-n) d(m+n)+\frac{m^{3}}{12} \delta_{m+n, 0} z, \quad[\mathscr{V} i r, z]=0 .
$$

Note the base $d(n)$ is obtained as $d(n):=l(n)-\delta_{n, 0} z / 24$ from $l(n)$ in (1). By this basis $\mathscr{V}_{i}$-characters $\operatorname{Tr} q^{d(0)}$ appearing in this paper enjoy the modular transformation property without the fractional power factor $q^{-z / 24}(\S 1.2 .2 \sim 3$; see also Example 5.3).

\subsection{Acknowledgement}

After the publication of the announce paper [Has], the author was informed by Professor V. Kac his work [KP1] with D. Peterson, in which Theorem AFF for the pair $\left(\mathfrak{s p}(2 l)^{\wedge}, \mathfrak{s p}(2 r)^{\wedge}\right)$ is obtained. See also Remark 4.2.

The present paper is originally designed for the Master's Thesis at Nagoya University under the supervision of Professor A. Tsuchiya and Professor Y. Kanie. The author expresses his sincere gratitude for their kind and continuous encouragement. He also thanks Professors T. Hayashi, R. Howe, M. Jimbo, V. Kac, T. Miwa and T. Umeda for discussions and/or informations.

\section{§1. Preliminaries for Classical Lie Algebras}

1.0. Here are brief summaries on affine Lie algebras and their outer automorphisms. We also list basic data and fix the notation for the classical simple Lie algebras and the classical affine Lie algebras following mainly [B] and [JM].

\subsection{Conventions}

Throughout this paper we work over the complex number field $\mathbb{C}$. We denote by $\mathbf{Z}$ (resp. $\mathbf{Z}_{>0}, \mathbb{Z}_{\geq 0}, \ldots$ ) the set of rational integers (resp. positive, nonnegative, .. rational integers). For a real number $r,[r] \in \mathbb{Z}$ is the maximum integer that does not exceed $r$. We write $\Xi_{n}\left(n \in \mathbb{Z}_{>0}\right)$ for the symmetric group of $n$ letters. By $\operatorname{Mat}(m, n)$ we denote the set of all $m \times n$-matrices, and $E_{j}^{i}(1 \leq i \leq m, 1 \leq j \leq n)$ the matrix unit. If $m=n$ they satisfy $E_{j}^{i} E^{k}{ }_{l}=\delta_{j}{ }^{k} E_{l}^{i}$, where $\delta_{j}{ }^{k}=\delta_{j k}=\delta^{j}{ }_{k}$ is the Kronecker delta, and act on $\mathbf{C}^{n}=\bigoplus_{k=1}^{n} \mathbf{C} v^{k}$ by $E_{j}^{i} v^{k}=v^{i} \delta_{j}^{k}$. We write $\operatorname{gl}(n)$ for the Lie algebra $\operatorname{Mat}(n, n)$ with 
the bracket $[A, B]:=A B-B A$. We put $\mathbb{1}_{n}:=\sum_{j=1}^{n} E_{j}^{j}$. When we have to deal with several Lie algebras $\mathfrak{g}, \mathfrak{f}, \ldots$ at the same time, we often use the notation such as $\mathbb{L}^{\mathfrak{g}}(\lambda), \mathbb{L}^{\mathfrak{f}}(\mu), \ldots ; \chi_{\lambda}^{\mathfrak{g}}, \chi_{\mu}^{\mathfrak{f}}, \ldots ; D^{\mathfrak{g}}(n), D^{\mathfrak{f}}(n), \ldots$ etc., to distinguish the notions accordingly.

\subsection{Alfine Kac-Moody Lie algebras}

We will follow Kac's book [Kac] for general terminologies.

\subsubsection{A}

Let $g$ be a finite dimensional reductive Lie algebra and $B(, \quad)$ a non-degenerate invariant symmetric bilinear form on $\mathfrak{g}$. Then the affinization $\mathfrak{g}^{\wedge}=\mathfrak{g}_{\hat{B}}^{\wedge}$ of $\mathfrak{g}$ is the $\mathbb{L i e}$ algebra defined by

$$
\begin{gathered}
\mathfrak{g}^{\wedge}:=\mathfrak{g} \otimes \mathbb{C}\left[t, t^{-1}\right] \oplus \mathbb{C} c, \\
{[x(m), y(n)]:=[x, y](m+n)+m \delta_{m+n, 0} B(x, y) \cdot c, \quad\left[c, \mathfrak{g}^{\wedge}\right]:=0,}
\end{gathered}
$$

where $x(m)$ denotes $x \otimes t^{m}$. We put $\mathfrak{g}(n):=\left\{X \otimes t^{n} \mid X \in \mathfrak{g}\right\}$ and identify $X \in \mathfrak{g}$ with $X(0) \in \mathfrak{g}(0) \subset \mathfrak{g}^{\wedge}$. There is an extention $\mathscr{V}_{i \imath} \times \mathfrak{g}^{\wedge}$ of the Virasoro algebra $\mathscr{V}_{i r}$ by $\mathfrak{g}^{\wedge}$, where

$$
\begin{gathered}
\mathscr{V}_{i r}:=\bigoplus_{h \in \mathbb{Z}} \mathbb{C} d(n) \oplus \mathbb{C} z, \\
{[d(m), d(n)]:=(m-n) d(m+n)+\frac{m^{3}}{12} \delta_{m+n, 0} z, \quad\left[z, \mathscr{V}_{i}\right]:=0,}
\end{gathered}
$$

and the action of $\mathscr{V}_{i \iota}$ on $\mathfrak{g}^{\wedge}$ is defined by

$$
[d(m), x(n)]:=-n \cdot x(m+n) \quad \text { and } \quad\left[z, \mathfrak{g}^{\wedge}\right]:=\left[\mathscr{V}_{i}, c\right]:=0 .
$$

(See 0.5 or 1.2 .3 for the reason why we take $m^{3} / 12$ in (2) instead of $\left(m^{3}-m\right) / 12$.)

Suppose that $\mathfrak{g}$ is simple and of type $X_{r}$. Then the subalgebra $\tilde{\mathfrak{g}}:=\mathbb{C} d(0) \ltimes \mathfrak{g}^{\wedge}$ of $\mathscr{V}_{i r} \ltimes \mathfrak{g}^{\wedge}$ is isomorphic to the affine Kac-Moody Lie algebra of type $X_{r}^{(1)}$. Its canonical central element (resp. scaling element $d$ ) is given by $c$ (resp. $-d(0)$ ). Here and after we choose the normalization $B(\theta, \theta)=2$ for a long root $\theta$ of $g$, and such $B$ 's will be given in 1.4. Taking a triangular decomposition $\mathfrak{g}=\mathfrak{n}_{-} \oplus \mathfrak{h} \oplus \mathfrak{n}_{+}$of $\mathfrak{g}$, we can give and fix a triangular decomposition $\tilde{\mathfrak{g}}=\tilde{\mathfrak{n}}_{+} \oplus \tilde{\mathfrak{h}} \oplus \tilde{\mathfrak{n}}_{-}$canonically by

$$
\tilde{\mathfrak{h}}:=\mathfrak{h} \oplus \mathbb{C} c \oplus \mathbb{C} d \quad \text { and } \quad \tilde{\mathfrak{n}}_{ \pm}:=\left(\mathfrak{g} \otimes t^{ \pm 1} \mathbb{C}\left[t^{ \pm 1}\right]\right) \oplus \mathfrak{n}_{ \pm} .
$$

Define $\hat{\Lambda}_{0}$ and $\delta \in \tilde{\mathfrak{h}}^{*}=(\mathfrak{h} \oplus \mathbb{C} c \oplus \mathbb{C} d)^{*}$ respectively by

$$
\left.\hat{\Lambda}_{0}\right|_{\mathfrak{h} \oplus \mathbb{C} d}=0, \quad \hat{\Lambda}_{0}(c)=1 \quad \text { and }\left.\quad \delta\right|_{\mathfrak{h} \oplus \mathbb{C} c}=0, \quad \delta(d)=1,
$$

and extend each $\lambda \in \mathfrak{h}^{*}$ to $\tilde{\mathfrak{h}}$ by $\left.\lambda\right|_{\mathbb{C} c \oplus \mathbb{C} d}=0$. We have $\tilde{\mathfrak{h}}^{*}=\mathfrak{h}^{*} \oplus \mathbb{C} \hat{\Lambda}_{0} \oplus \mathbb{C} \delta$. We extend $B$ to $\tilde{\mathfrak{h}}^{*}$ by the formula

$$
B\left(\lambda+l \hat{\Lambda}_{0}+s \delta, \mu+m \hat{\Lambda}_{0}+t \delta\right):=B(\lambda, \mu)+l t+s m
$$

for $\lambda, \mu \in \mathfrak{h}^{*}$ and $l, m, s, t \in \mathbb{C}$. 
Let $\Lambda_{j}$ and $a_{j}^{\vee}$ be the fundamental weights of $\mathfrak{g}\left(\right.$ not $\left.\mathrm{g}^{\wedge}\right)$ and the dual labels of $X_{r}^{(1)}$ respectively (see 1.4 and 1.5 for their tables). Then the fundamental weights of $\mathrm{g}^{\wedge}$ are given by

$$
\hat{\Lambda}_{0} \quad \text { and } \quad \hat{\Lambda}_{j}:=a_{j}^{\vee} \hat{\Lambda}_{0}+\Lambda_{j} \quad(1 \leq j \leq r)
$$

and the set of dominant integral weights is given by $\hat{P}_{+}+\mathbb{C} \delta$, where $\hat{P}_{+}:=\sum_{j=0}^{r} \mathbb{Z}_{\geq 0} \hat{\Lambda}_{j}$. We put $\hat{P}_{+}(l):=\left\{\Lambda \in \hat{P}_{+} \mid \Lambda(c)=l\right\}$. Also we put $\rho:=\sum_{j=1}^{r} \Lambda_{j} \in \mathfrak{h}^{*}$ and $\hat{\rho}:=\sum_{j=0}^{r} \hat{\Lambda}_{j}=$ $\rho+g \hat{\Lambda}_{0}$, where $g$ is the dual Coxeter number $g=\hat{\rho}(c)=\Sigma_{j} a_{j}^{\vee}$.

If $\mathfrak{g}$ is abelian, then in what follows we regard $\mathfrak{h}=\mathfrak{g}, \rho=0, \hat{\rho}=0$ and $g=0$.

\subsubsection{The Segal-Sugawara operators}

Assume that $\mathrm{g}$ is simple or abelian, and that $V$ is a $\mathfrak{g}^{\wedge}$-module that satisfies the following two conditions.

(1) For any $v \in V$, there exists an integer $m \in \mathbb{Z}_{\geq 0}$ such that $\mathfrak{g}(n) v=0$ whenever $n>m$.

(2) $c=l \cdot i d_{V}$ for some $l \in \mathbb{C}$ such that $l+g \neq 0$,

The scalar $l$ is called the level of $V$. Then it is well known that the following Segal-Sugawara operator $D^{\hat{\mathrm{g}}}(n) \in$ End $V$ gives a $\left(\mathscr{V}_{i} \ltimes \ltimes \hat{\mathrm{g}}\right)$-module structure on $V$ :

$$
\begin{gathered}
\mathscr{V} i r \ni d(n) \mapsto D^{\hat{\mathrm{g}}}(n):=\frac{1}{2(l+g)} \sum_{j=1}^{\operatorname{dim} \mathrm{g}} \sum_{m \in \mathbb{Z}}{ }_{\circ} x_{j}(m) x^{j}(n-m) \circ-\frac{l \cdot \operatorname{dim} \mathrm{g}}{24(l+g)} \delta_{n, 0} i d_{V}, \\
z \mapsto z^{\hat{\mathrm{g}}}:=\frac{l \cdot \operatorname{dim} \mathfrak{g}}{l+g} i d_{V} .
\end{gathered}
$$

Here $\left(\left\{x_{j}\right\},\left\{x^{j}\right\}\right)$ is a dual basis of $g$ with respect to $B$ and

$$
: x(m) y(n) \circ:= \begin{cases}x(m) y(n) & \text { if } m<n \\ \{x(m) y(n)+y(n) x(m)\} / 2 & \text { if } m=n . \\ y(n) x(m) & \text { if } m>n\end{cases}
$$

If $\mathfrak{g}=\bigoplus \mathfrak{g}_{i}$ with each $\mathfrak{g}_{i}$ is either simple or abelian, then we put

$$
D^{\hat{\mathrm{g}}}(m):=\Sigma_{i} D^{\hat{\mathrm{g}}_{\imath}}(m) \quad \text { and } \quad z^{\hat{\mathrm{g}}}:=\Sigma_{i} z^{\hat{\mathrm{g}}_{i}} .
$$

\subsubsection{Irreducible modules and characters}

An irreducible highest weight module of $\mathrm{g}^{\wedge}$ with the highest weight vector $v_{0}$ is characterized by $(\lambda ; l) \in \mathfrak{h}^{*} \times \mathbb{C}$ such that

$$
h\left(v_{0}\right)=\lambda(h) v_{0}\left({ }^{\forall} h \in \mathfrak{h}\right) \quad \text { and } \quad c\left(v_{0}\right)=l \cdot v_{0} .
$$

We call $\lambda$ (resp. $l$ ) the classical part (resp. level) of the highest weight and denote such a module by $\mathbb{L}^{\hat{g}}(\lambda ; l)$. Provided that $l+g \neq 0$, then $\mathbb{L}^{\hat{\mathrm{g}}}(\lambda ; l)$ satisfies $1.2 .2(1)(2)$ and admits a $\left(\mathscr{V}_{i} \measuredangle \times \mathfrak{g}^{\wedge}\right)$-module structure. In particular we can (and do) fix a $\tilde{\mathrm{g}}$-module structure canonically. Namely, for $\lambda \in \mathfrak{h}^{*}$ and $l(\neq-g) \in \mathbb{C}$ we will identify $\mathbb{L}^{\hat{g}}(\lambda ; l)$ with the $\tilde{\mathrm{g}}$-irreducible highest weight module: 


$$
\mathbb{L}^{\hat{\mathrm{g}}}(\lambda ; l)=\mathbb{L}^{\tilde{g}}\left(\lambda+l \hat{\Lambda}_{0}+\left(\frac{l \cdot \operatorname{dim} \mathfrak{g}}{24(l+g)}-\frac{|\lambda+\rho|^{2}-|\rho|^{2}}{2(l+g)}\right) \delta\right),
$$

according to the fact

$$
d v_{0}\left[:=-D^{\hat{g}}(0) v_{0}\right]=\left(\frac{l \cdot \operatorname{dim} \mathrm{g}}{24(l+g)}-\frac{|\lambda+\rho|^{2}-|\rho|^{2}}{2(l+g)}\right) v_{0} .
$$

From the famous strange formula it follows that

$$
\frac{l \cdot \operatorname{dim} g}{24(l+g)}-\frac{|\lambda+\rho|^{2}-|\rho|^{2}}{2(l+g)}=\frac{|\rho|^{2}}{2 g}-\frac{|\lambda+\rho|^{2}}{2(l+g)} .
$$

Suppose $V$ is a diagonalized $\mathbb{C} d(0)$-module, that is,

$$
V=\bigoplus_{m} V_{m}, \quad \operatorname{dim} V_{m}<\infty
$$

where $V_{m}:=\{v \in V \mid d(0) v=m v\}$ for $m \in \mathbb{C}$. Then for $\tau \in \mathbb{C}$ and an operator $u \in$ End $V$ that satisfy $[d(0), u]=0$, we define the character of $V$ formally by

$$
\operatorname{ch}_{V}(\tau d(0)+\mathrm{u}):=\operatorname{ch}_{V}(\tau, \mathrm{u}):=\Sigma_{m} \mathrm{e}[m \tau] \operatorname{Trace}_{V_{m}} e^{2 \pi i \mathrm{u}},
$$

where $\mathbf{e}[*]:=e^{2 \pi i *}$, and put $\chi_{\lambda, l}^{\hat{\mathrm{g}}}:=\operatorname{ch}_{\mathbf{L} \hat{(}(\lambda ; l)}$ for short. According to our choice of the cocycle in the definition 1.2.1(2) of $\mathscr{V}_{i} i$, we have (2) (3) above and hence $\chi^{\hat{\mathrm{g}}}{ }_{\lambda, l}$ is nothing but the so-called normalized character. It converges for $\tau \in \mathbb{C}, \operatorname{Im} \tau>0$ and $\mathbb{u} \in \mathfrak{h}$.

If $\mathfrak{g}$ is simple, then for a dominant integral weight $\lambda+l \hat{\Lambda}_{0} \in \hat{P}_{+}$we have the celebrated Weyl-Kac formula

$$
\chi_{\lambda, l}^{\hat{\mathrm{g}}}(\tau, \mathrm{u})=\mathscr{A}_{\lambda+\rho, l+g}(\tau, \mathrm{u}) / \mathscr{A}_{\rho, g}(\tau, \mathrm{u}) .
$$

Here denoting by $W$ (resp. $M$ ) the Weyl group of $\mathfrak{g}$ (resp. the lattice part of the affine Weyl group $\hat{W}=W \ltimes M$ ), we put

$$
\theta_{\lambda, l}^{M}(\tau, \mathbf{u}):=\Sigma_{\mu \in \lambda+l M} \mathbf{e}\left[\mu(\mathbf{u})+\tau|\mu|_{B}^{2} / 2 l\right]
$$

and

$$
\mathscr{A}_{\lambda, l}:=\Sigma_{w \in W}(\operatorname{sgn} w) \theta_{w(\lambda), l}^{M} .
$$

For the latter purpose we also put

$$
\chi_{\Lambda}^{\hat{\mathrm{g}}}:=\chi_{\lambda, l}^{\hat{\mathrm{g}}}, \quad \theta_{\Lambda}^{M}:=\theta_{\lambda, l}^{M} \quad \text { and } \quad \mathscr{A}_{\Lambda}:=\mathscr{A}_{\lambda, l}
$$

for a weight $\Lambda \in \tilde{\mathfrak{h}}^{*}$ of the form

$$
\Lambda \equiv \lambda+l \hat{\Lambda}_{0} \bmod \mathbb{C} \delta, \quad l>0 .
$$

The denominator formula says that $\mathscr{A}_{\rho, g}=\mathscr{A}_{\hat{\rho}}$ has a product form $\mathscr{D}$, which is given explicitly in 1.5 for the classical non-twisted affine Lie algebras.

If $\mathrm{g}$ is abelian, then for $l \in \mathbb{C}^{\times}$and $\lambda \in \mathrm{g}^{*}$ we have ([FK])

$$
\chi_{\lambda, l}^{\mathrm{q} \wedge}(\tau, \mathrm{u})=\mathrm{e}[\lambda(\mathrm{u})] \eta(\tau)^{-\operatorname{dim} \mathrm{g}} \quad(\mathrm{u} \in \mathrm{g}),
$$

where $\eta$ is the Dedekind eta (1.5.1(14)). See also 1.3.3. 


\subsubsection{Complete reducibility and the coset $\mathscr{V}_{i} i$-modules}

The following lemma ensures complete reducibility throughout this article.

Lemma 1.2(i) [Kac, Theorem 10.7a]. Suppose $\mathrm{g}$ is simple and $e_{i} \in \tilde{\mathrm{n}}_{+}$denotes the Chevalley generator of $\mathfrak{g}^{\wedge}$. Let $V$ be an integrable $\mathfrak{g}^{\wedge}$-module which satisfies that for any $v \in V$, there exists $m \in \mathbb{Z}_{>0}$ so that $e_{i_{1}} \ldots e_{i_{n}} v=0$ whenever $n>m$. Then $V$ is isomorphic to a direct sum of $\mathbb{L}^{\hat{\mathrm{g}}}(\Lambda)$ for $\Lambda \in \hat{P}_{+}$.

(ii) [Kac, Lemma 14.4b]. Suppose $\mathfrak{g}$ is abelian, and $\hat{\mathrm{g}}^{\times} \subset \mathfrak{g}^{\wedge}$ the subalgebra $\hat{\mathfrak{g}}^{\times}:=\mathfrak{g}^{\wedge-} \oplus \mathbb{C} c \oplus \mathfrak{g}^{{ }^{+}}$, where $\mathfrak{g}^{\wedge \pm}:=\mathfrak{g} \otimes t^{ \pm 1} \mathbb{C}\left[t^{ \pm 1}\right]$. Let $V$ be a $\mathfrak{g}^{\wedge \times}$-module that satisfies 1.2.2(2) and for any $v \in V$, there exists $m>0$ so that $\mathfrak{g}\left(n_{1}\right) \ldots \mathfrak{g}\left(n_{j}\right) v=0$ provided that $n_{i}>0(1 \leq i \leq j)$ and $\Sigma n_{i}>m$. Then $V$ is isomorphic to a direct sum of the irreducible module $U\left(\mathfrak{g}^{\wedge \times}\right) / U\left(\mathfrak{g}^{\wedge \times}\right)\left(\mathfrak{g}^{\wedge+} \oplus \mathbb{C}(c-l)\right)$ for $l \in \mathbb{C}^{\times}$.

Let $\mathfrak{g}^{\wedge}$ be an affinization of a reductive Lie algebra $\mathfrak{g}$ and let $\pi$ and $\pi^{\prime}$ respectively be representations of $\mathscr{V}_{i}$ and $\mathfrak{g}^{\wedge}$ on the same space $V$. Suppose that their central elements acts as $\pi(z)=z^{\pi} \cdot i d_{V}$ and $\pi^{\prime}(c)=l \cdot i d_{V}$ for some $z^{\pi}, l \in \mathbf{C}$, and that

$$
\left[\pi(d(m)), \pi^{\prime}(x(n))\right]=-n \cdot \pi^{\prime}(x(m+n))
$$

holds for any $m, n \in \mathbb{Z}$. This means

$$
\left[\pi(d(m))-D^{g^{\wedge}}(m), \mathfrak{g}^{\wedge}\right]=0
$$

and $D^{\perp}(m):=\pi(d(m))-D^{g^{\wedge}}(m)$ again defines a representation of $\mathscr{V} i r$ on the branching module (Definition 0.2)

$$
\begin{gathered}
\mathbb{B}^{\Lambda}:=\operatorname{Hom}_{\mathfrak{g}^{\wedge}}\left(\mathbb{L}^{\mathfrak{g}^{\wedge}}(\Lambda), V\right) \\
\simeq\left\{v \in V \mid\left(\tilde{\mathfrak{n}}^{+}\right) v=0 \text { and } v \text { is of weight } \Lambda\right\},
\end{gathered}
$$

if we assume the complete reducibility

$$
V \simeq \bigoplus_{\Lambda \in \boldsymbol{P} \wedge(l)} \mathbb{B}^{\Lambda} \otimes \mathbb{L}^{\mathfrak{g}^{\wedge}}(\Lambda)
$$

This is the Goddard-Kent-Olive construction of the coset $\mathscr{V}_{i r}$-modules.

Proposition 1.2. We retain the above situation and moreover assume that the character $\operatorname{ch}_{V}(\tau)$ converges for $\operatorname{Im} \tau>0$. Then the following three conditions are equivalent:

(i) $V$ is finitely reducible as a $\mathfrak{g}^{\wedge}$-module,

(ii) $z^{\pi}=z^{\mathfrak{g}^{\wedge}}$,

(iii) $\pi(d(m))=D^{\hat{\hat{f}}}(m)$ for all $m \in \mathbb{Z}$.

Proof. Consider the coset representation $D^{\perp}(m)$. Then (i) $\rightarrow$ (ii) follows from the fact that any finite dimensional $\mathscr{V}_{i} \iota$-module must be trivial. Similarly (ii) $\rightarrow$ (iii) follows from that any unitarizable highest weight module with the central charge ( $=$ the eigenvalue of $z$ ) zero is trivial. For (iii) $\rightarrow$ (i) we consider the character. Using (iii), we obtain

$$
\infty>\operatorname{ch}_{V}\left(\pi(d(0))=\Sigma_{\Lambda \in P_{+}^{\wedge}(l)}\left(\operatorname{dim} \mathbb{B}^{\Lambda}\right) \chi^{\hat{\mathrm{g}}}{ }_{\Lambda}\left(\tau D^{\hat{\mathrm{g}}}(0)\right),\right.
$$

which shows $\operatorname{dim} \mathbb{B}^{\Lambda}<\infty$ for each $\Lambda$. 


\subsection{Modules which admit æetions of outer æutomorphisms}

\subsubsection{Didagram automorphisms}

Let $g(X)^{\prime}$ be the derived algebra of the $\mathbb{K a c}-\mathbb{M}$ loody algebra $\mathfrak{g}(X)$ for a generalized Cartan matrix $X=\left(x_{i, j}\right)_{0 \leq i, j \leq l}$. Then $\mathfrak{g}(X)^{\prime}$ has the Chevalley generators $\left\{e_{i}, f_{i}, h_{i}\right\}_{0 \leq i, j \leq l}$ that satisfy the following defining relations:

$$
\begin{gathered}
{\left[h_{i}, h_{j}\right]=0, \quad\left[h_{i}, e_{j}\right]=x_{i j} e_{j}, \quad\left[h_{i}, f_{j}\right]=-x_{i j} f_{j}, \quad\left[e_{i} f_{j}\right]=\delta_{i j} h_{j},} \\
\left(\operatorname{ad} e_{i}\right)^{1-x_{i j}} e_{j}=\left(\operatorname{ad} f_{i}\right)^{1-x_{i}} f_{j}=0 \quad(i \neq j) .
\end{gathered}
$$

Suppose $\left(x_{i j}\right)$ is invariant under a permutation $w$ of indices, that is, $w$ is a symmetry of the Dynkin diagram of $X$. Then $w$ induces an automorphism $\sigma_{w}$ of $\mathfrak{g}(X)^{\prime}$ by

$$
\sigma_{w}: g_{i} \mapsto g_{w(i)} \quad \text { for } \quad g=e, f, h \quad(1 \leq i \leq l),
$$

since this assignation preserves the defining relations. We call the automorphism thus obtained a diagram automorphism. Note that $\sigma_{w}$ preserves the triangular decomposition of $g(X)^{\prime}$. Note also that (the adjoint of) $\sigma_{w}$ acts on the roots and their linear combinations by

$$
\sigma_{w}: \alpha_{i} \mapsto \alpha_{w(i)} \quad \text { for a simple root } \alpha_{i}
$$

Lemma 1.3.1. Suppose $X$ is of affine type. Then the above $\sigma_{w}$ has a unique lifting to the automorphism of $\mathfrak{g}(X)$ and $\tilde{\mathfrak{h}}^{*}$ (also denoted by $\sigma_{w}$ ) that satisfies the following conditions.

(4) $\left.\sigma_{w}\right|_{g_{(X)}}$ and $\left.\sigma_{w}\right|_{\mathfrak{h}^{*} \oplus \mathbf{C} \delta}$ are given respectively by (2) and (3),

(5) $\sigma_{w}(\tilde{\mathfrak{h}}) \subset \tilde{\mathfrak{h}}$ and $\left\langle\sigma_{w}(\alpha), \sigma_{w}(h)\right\rangle=\langle\alpha, h\rangle$ for any $\alpha \in \tilde{\mathfrak{h}}^{*}$ and $h \in \tilde{\mathfrak{h}}$,

(6) the order of $\sigma_{w}$ is equal to that of $w$.

Proof. As usual we assume that 0 is the index of affine point in the Dynkin diagram. Recall that $\delta=\sum_{i=0}^{l} a_{i} \alpha_{i}$ and $c=\sum_{i=0}^{l} \check{a}_{i} h_{i}$, where $a_{i}$ (resp. $\check{a}_{i}$ ) is the $i$-th label (resp. dual label) for $X$ ([Kac]). Therefore $\tilde{\mathfrak{h}}^{*}=\bigoplus_{i=0}^{l} \mathbb{C} \alpha_{i} \oplus \mathbb{C} \hat{\Lambda}_{0}$ and $\tilde{\mathfrak{h}}=\bigoplus_{i=0}^{l} \mathbb{C} h_{i} \oplus \mathbb{C} d$. It suffices to determine $\sigma_{w}(d) \in \tilde{\mathfrak{h}}$ so that (5) and (6) holds, since then (5) defines $\sigma_{w}\left(\hat{\Lambda}_{0}\right)$ and the $\sigma_{w}$ thus extended automatically gives rise to an automorphism of $\mathfrak{g}(X)$. Since $a_{i}=a_{w(i)}$ it holds that $\sigma_{w}(\delta)=\delta$ and hence we can assume

$$
\sigma_{w}(d)=d+\sum_{i=0}^{l} s_{i} h_{i}
$$

with $s_{i} \in \mathbb{C}$. The condition (5), $\left\langle\sigma_{w}^{-1}\left(\alpha_{i}\right), d\right\rangle=\left\langle\alpha_{i}, \sigma_{w}(d)\right\rangle$, then reads as $\delta_{i, w(0)} a_{0}^{-1}=$ $\delta_{i, 0} a_{0}^{-1}+\Sigma_{j} s_{j} x_{j, i}$, or

$$
\Sigma_{j} s_{j} x_{j, i}=a_{0}^{-1}\left(\delta_{i, w(0)}-\delta_{i, 0}\right) \quad(0 \leq i \leq l) .
$$

Similarly (6) reads as

$$
\sum_{k=1}^{\operatorname{ord}(w)} s_{w^{k}(i)}=0 \quad(0 \leq i \leq l) .
$$

Now our task is to solve (8) and (9), and the case checking shows that there exists a unique solution $\left\{s_{i}\right\}$ for them in each case. 
For $X=A_{l}^{(1)}, B_{l}^{(1)}, C_{l}^{(1)}$ and $D_{l}^{(1)}$, we give in $\S 1.5$ the formulae of actions of the diagram automorphisms on $\tilde{\mathfrak{h}}$ and $\tilde{\mathfrak{h}}^{*}$.

Remark 1.3.1. (i) The condition (6) is not redundant.

(ii) If $w(0) \neq 0$, then the equality $\sigma_{w}\left(\hat{\Lambda}_{j}\right)=\hat{\Lambda}_{w(j)}$ is not always true (however $\left.\sigma_{w}\left(\hat{\Lambda}_{j}\right) \equiv \hat{\Lambda}_{w(j)} \bmod \mathrm{C} \delta\right)$. On the other hand, it holds that $\sigma_{w}(\hat{\rho})=\hat{\rho}$.

\subsection{2. $\Sigma \times g(X)$-modenles}

Let $X$ be a generalized Cartan matrix and $\Sigma \subset$ Aut $_{L i e} g(X)$ be a group consisting of diagram automorphism (1.3.1).

Definition 1.3.2. (i) We say a $\mathfrak{g}(X)$-module $(\pi, V)$ is a $\Sigma \times \mathfrak{g}(X)$-module if there exists a projective representation $\pi^{\prime}$ of $\Sigma$ on $V$ which satisfies the compatibility condition

$$
\pi^{\prime}(\sigma) \pi(g) \pi^{\prime}(\sigma)^{-1}=\pi(\sigma(g))
$$

for all $\sigma \in \Sigma$ and $g \in \mathfrak{g}(X)$. (The notation $\Sigma \ltimes \mathfrak{g}(X)$ is justified if we think it as an abbreviation of the semi-direct product algebra $\mathbb{C}[\Sigma] \ltimes U(\mathfrak{g}(X))$ which is generated by $\Sigma$ and $\mathfrak{g}(X)$.)

(ii) For a weight $\lambda$, we define a $g(X)$-module

$$
\mathbf{L}^{\Sigma \times \mathbf{g}(X)}(\lambda):=\bigoplus_{\mu \in \Sigma \cdot \lambda} \mathbb{L}^{\mathrm{g}(X)}(\mu),
$$

where $\Sigma \cdot \lambda$ denotes the $\Sigma$-orbit of $\lambda$. This module gives rise to an irreducible $\Sigma \ltimes \mathfrak{g}(X)$-module by defining

$$
\pi^{\prime}(\sigma)|\mu\rangle:=|\sigma(\mu)\rangle \quad \text { for any } \quad \sigma \in \Sigma,
$$

where $|\mu\rangle$ denotes the highest weight vector of $\mathbb{L}^{\mathbf{g}(X)}(\mu)$.

We also define the character

$$
\chi_{\lambda}^{\Sigma \times g(X)}:=\Sigma_{\mu \in \Sigma \cdot \lambda} \chi_{\mu}^{g(X)}
$$

accordingly.

Remark 1.3.2. (i) The module $\mathbb{L}^{\Sigma \times g(X)}(\lambda)$ does not always coincide with the module $\bigoplus_{\sigma \in \Sigma} \mathbb{L}^{g(X)}(\sigma(\lambda))$.

(ii) For the Cartan matrix $X$ of type $D_{n}, \mathfrak{g}(X) \simeq \mathfrak{o}(2 n)$ holds and the group of diagram automorphisms is generated by the element $\sigma=\sigma_{n-1, n}(1.4 .5(0))$ or order two. In this case the module $\mathbb{L}^{\Sigma \times g(X)}(\lambda)$ for a dominant integral weight $\lambda$ is nothing but a finite dimensional irreducible $\operatorname{Pin}(2 n)$-module, while $\mathbb{L}^{g(X)}(\lambda)$ is an irreducible $\operatorname{Spin}(2 n)$ module. Here $\operatorname{Pin}(2 n)$ and $\operatorname{Spin}(2 n)$ denotes the double covering of the orthogonal group $\mathrm{O}(2 n)$ and $\mathrm{SO}(2 n)$, respectively.

Lemma 1.3.2. Suppose that a $\Sigma \times \mathfrak{g}(X)$-module $V$ is completely reducible as a $\mathfrak{g}(X)$-module, and $\Sigma$ is abelian. Then $V$ decomposes into a direct sum of $\mathbb{L}^{\Sigma \times g(X)}(\lambda)$ 's, that is, there are modules $\mathbb{B}^{\lambda}$ 's such that

as a $\Sigma \ltimes \mathfrak{g}(X)$-module.

$$
V \simeq \bigoplus_{\lambda}\left(\mathbb{B}^{\lambda} \otimes \mathbb{L}^{\Sigma \times g(X)}(\lambda)\right)
$$


Proof. A $\Sigma \ltimes \mathfrak{g}(X)$-submodule of $V$ and a $(\Sigma, \mathfrak{h})$-submodule of $H W V(V):=$ $\left\{v \in V \mid\left(\mathrm{n}_{+}\right)^{g(X)} v=0\right\}$ are in one to one correspondence with each other, where $\left(\mathrm{n}_{+}\right)^{g(X)}$ denotes the subalgebra of $\mathfrak{g}(X)$ generated by the Chevalley generators $\left\{e_{i}\right\}$. Since $H W V(V)$ has a weight space decomposition and $\Sigma$ is a finite group, $H W V(V)$ can be decomposed into a direct sum of irreducible $(\Sigma, \mathfrak{h})$-modules. Then each of the irreducible component is restricted its form as $\bigoplus_{\mu \in \Sigma \cdot \lambda} \mathbb{C}|\mu\rangle$ under the assumption that $\Sigma$ is abelian. Accordingly, the corresponding irreducible $\Sigma \ltimes g(X)$-submodule of $V$ is of the form $\mathbb{L}^{\Sigma \times g(X)}(\lambda)$, which completes the proof.

\subsubsection{Review on Heisenberg systems $[F \mathrm{~K} \rrbracket$}

Let $\mathfrak{a}$ be an abelian $\mathbb{L i e}$ algebra, $B$ the non-degenerate symmetric bilinear form on $\mathfrak{a}$ and $\mathfrak{a}^{\wedge}=\mathfrak{a}_{\boldsymbol{B}}^{\wedge}$ the corresponding affinization (1.2.1(1)). Then the subalgebra $\mathfrak{a}^{\wedge \times}:=\bigoplus_{m \neq 0} \mathfrak{a}(m) \oplus \mathbb{C} c$ is an infinite Heisenberg Lie algebra and we have a direct sum decomposition $\mathfrak{a}^{\wedge}=\mathfrak{a} \oplus \mathfrak{a}^{\wedge \times}$ as a Lie algebra. Let $\Gamma \subset \mathfrak{a}^{*}$ be a lattice, then each $\gamma \in \Gamma$ act on $\mathfrak{a}^{\wedge}$ by

$$
\gamma\left(a_{0}+a_{\times}\right):=a_{0}+a_{\times}-\gamma\left(a_{0}\right) c
$$

for any $a_{0} \in \mathfrak{a}$ and $a_{\times} \in \mathfrak{a}^{\wedge \times}$. We call this system $\Gamma \ltimes \mathfrak{a}^{\wedge}=\left(\Gamma, \mathfrak{a}^{\wedge}\right)$ a Heisenberg system. An $\mathfrak{a}^{\wedge}$-module is said to be a $\Gamma \ltimes \mathfrak{a}^{\wedge}$-module if there exists a projective action of $\Gamma$ satisfying the compatibility 1.3.2(1).

A typical $\Gamma \propto \mathfrak{a}^{\wedge}$-module is the Boson Fock space

$$
\begin{aligned}
\mathbb{L}^{\Gamma \times \mathfrak{a}^{\wedge}}(\lambda ; l) & :=\left(\bigoplus_{\alpha \in \lambda+l \Gamma} \mathbb{C} e^{\alpha}\right) \otimes\left(U\left(\mathfrak{a}^{\wedge \times}\right) / U\left(\mathfrak{a}^{\wedge \times}\right)\left(\mathbb{C}(c-l) \oplus \mathfrak{a}^{\wedge+}\right)\right) \\
& \simeq e^{\lambda} \mathbb{C}[l \Gamma] \otimes S\left(\mathfrak{a}^{\wedge-}\right),
\end{aligned}
$$

where $\lambda \in \mathfrak{a}^{*}, l \in \mathbb{C}^{\times}, \mathfrak{a}^{\wedge \pm}:=\mathfrak{a} \otimes t^{ \pm 1} \mathbb{C}\left[t^{ \pm 1}\right]$ and $\mathbb{C}[\cdot]$ (resp. $\left.S(\cdot)\right)$ denotes the group algebra (resp. symmetric algebra). For each cocycle

$$
\varepsilon: \Gamma \times \Gamma \rightarrow \mathbb{C}^{\times}, \quad \varepsilon(\alpha, \beta) \varepsilon(\alpha+\beta, \gamma)=\varepsilon(\alpha, \beta+\gamma) \varepsilon(\beta, \gamma),
$$

the corresponding $\Gamma \ltimes \hat{\mathrm{a}}$-action on $\mathbb{L}^{\Gamma \ltimes \mathfrak{a}^{\wedge}}(\lambda ; l) \simeq e^{\lambda} \mathbb{C}[l \Gamma] \otimes S\left(\mathfrak{a}^{\wedge-}\right)$ is given by the following way:

$$
\begin{aligned}
\Gamma \ni \gamma \mapsto T_{\gamma}^{\varepsilon}:=\left\{e^{\alpha} \otimes p \mapsto \varepsilon(\gamma, \beta) e^{\alpha+l \gamma} \otimes p\right\}, \\
\mathfrak{a} \ni a \mapsto \partial / \partial a:=\left\{e^{\alpha} \otimes p \mapsto \alpha(a) e^{\alpha} \otimes p\right\}, \\
\hat{\mathfrak{a}}^{-} \ni a(-m) \mapsto a(-m) \cdot:=\left\{e^{\alpha} \otimes p \mapsto e^{\alpha} \otimes a(-m) p\right\}, \\
\hat{\mathfrak{a}}^{+} \ni a(m) \mapsto l m \cdot \partial / \partial a(-m):=\left\{e^{\alpha} \otimes p \mapsto e^{\alpha} \otimes(l m \cdot \partial p / \partial a(-m))\right\},
\end{aligned}
$$

where $\alpha=\lambda+l \beta \in \lambda+l \Gamma, p \in S\left(\hat{\mathfrak{a}}^{-}\right), a \in \mathfrak{a}, m \in \mathbb{Z}_{>0}$ and $\partial / \partial a(-m)$ is the derivation defined by $a^{\prime}(-n) \mapsto \delta_{m, n} B\left(a, a^{\prime}\right)$.

If $l \neq 0$, then as is well known $\mathbb{L}^{\Gamma \propto \mathfrak{a}^{\wedge}}(\lambda, l)$ is irreducible ([FK, Proposition 2.4]). By the definition (2) and the relation $D^{\mathfrak{a}^{\wedge}}(0)\left(e^{\lambda}\right)=(B(\lambda, \lambda) / 2 l) e^{\lambda}(1.2 .2(3))$, its character is formally given by the theta series 


$$
\operatorname{ch}_{\mathbf{L}^{\Gamma \ltimes a}(\lambda, l)}\left(\tau D^{\mathfrak{a}^{\wedge}}(0)+a\right)=\frac{\theta_{\lambda, l}^{\Gamma}(\tau, a)}{\eta(\tau)^{\operatorname{dim} \mathfrak{a}}},
$$

where $a=a(0) \in \mathfrak{a}$ and $\theta_{\lambda, l}^{\Gamma}(\operatorname{resp} . \eta(\tau))$ is given by 1.2.3(8) (resp. 1.5.1(14)).

\subsection{4. $\mathrm{gI}(l)^{\wedge}$-modules}

For $\mathfrak{g l}(l)^{\wedge}$ we need the notions similar to that for $\mathfrak{g}(X)$ in $\S 1.3 .2$, namely $\left\langle\sigma_{c y c}^{\#}\right\rangle \ltimes \mathrm{gl}(l)^{\wedge}$-modules and $\mathbf{Z}^{l} \ltimes \mathrm{gl}(l)^{\wedge}$-modules. Here and after, by $\mathrm{gl}(l)^{\wedge}$ we mean the affinization of $\mathfrak{g l}(l)$ associated to the invariant symmetric form $B(x, y):=\operatorname{Tr}_{\mathbf{C}^{l}}(x y)$.

Definition 1.3.4a $\left(\sigma_{c y c}^{\#} \in \operatorname{Aut}_{L i e} \operatorname{gl}(l)^{\wedge}\right)$. We put

$$
\left\{\begin{array}{c}
\sigma_{c y c}^{\#}\left(E_{j}^{i}(n)\right):=E_{j+1(l)}^{i+1(l)}\left(n-\delta_{i l}+\delta_{j l}\right)-\delta_{n 0} \delta_{i j} \delta_{j l} \cdot c \\
\sigma_{c y c}^{\#}(c):=c
\end{array},\right.
$$

where $i+1(l)$ means $i+1 \bmod l$.

It holds that

$$
\begin{aligned}
\left.\sigma_{c y c}^{\#}\right|_{s \mathrm{I}(l)^{\wedge}}= & \sigma_{c y c}: \text { the diagram automorphism of } \mathfrak{g}\left(A_{l-1}^{(1)}\right) \\
& \text { associated to the cycle } c y c:=(0, \ldots, l-1),
\end{aligned}
$$

and

$$
\sigma_{c y c}^{\#}(1(n))=\mathbb{1}(n)-\delta_{n, 0} \cdot c,
$$

where $\mathbf{1}=\mathbb{1}_{l}:=\sum_{j=1}^{l} E_{j}^{j} \in \operatorname{gl}(l)$ and $\mathbf{1}(n)$ is an element of the Heisenberg algebra $\left(\mathbf{C 1}_{l}\right)^{\wedge} \subset \mathrm{gl}(l)^{\wedge}$. From the latter it follows that $\sigma_{c y c}^{\#}$ is of infinite order.

Definition 1.3.4b $\left(\left\langle\sigma_{c y c}^{\#}\right\rangle \times \operatorname{gl}(l)^{\wedge}\right.$-modules $)$.

(i) Let $(\pi, V)$ be a $g l(l)^{\wedge}$-module. We say $(\pi, V)$ a $\left\langle\sigma_{c y c}^{\#}\right\rangle \ltimes \operatorname{gl}(l)^{\wedge}$-module if there is a projective action $\pi^{\prime}$ of $\left\langle\sigma_{c y c}^{\#}\right\rangle$ with the compatibility 1.3.2(1).

(ii) For $(\mu, t ; r) \in \mathfrak{h}^{*} \times \mathbf{C} \times \mathbf{C}$, we define a $\mathfrak{g l}(l)^{\wedge}$-module $\mathbf{L}^{\mathrm{gl}(l)^{\wedge}}(\mu, t ; r)$ to be the irreducible $\operatorname{gl}(l)^{\wedge}$-module with the highest weight vector $|\mu, t ; r\rangle \neq 0$ that satisfies the followings:

$$
\begin{aligned}
& h(0)|\mu, t ; r\rangle=\mu(h)|\mu, t ; r\rangle \quad \text { for } \quad h \in \bigoplus_{j} \mathbf{C}\left(E^{j}{ }_{j}-E^{j+1}{ }_{j+1}\right) \text {, } \\
& \mathbf{1}_{l}(0)|\mu, t ; r\rangle=t \cdot|\mu, t ; r\rangle, \quad c|\mu, t ; r\rangle=r|\mu, t ; r\rangle
\end{aligned}
$$

and

$$
E_{j}^{i}(n)|\mu, t ; r\rangle=0 \quad \text { if } \quad n>0 \quad \text { or } \quad(n=0, i<j) .
$$

(iii) Putting

$$
\sigma_{c y c}^{\#} \cdot(\lambda, s ; r):=\left(\sigma_{c y c}(\lambda), s+r ; r\right)
$$

we define

$$
\mathbf{L}^{\left\langle\sigma_{c y c}^{\#}\right\rangle \times \operatorname{gl}(l)^{\wedge}}(\lambda, s ; r):=\bigoplus_{(\mu, t ; r) \in\left\langle\sigma_{c y c}^{\#}\right\rangle \cdot(\lambda, s ; r)} \mathbf{L}^{\mathfrak{g l}(l)^{\wedge}}(\mu, t ; r)
$$


and we endow a $\left\langle\sigma_{c y c}^{\#}\right\rangle \ltimes \mathfrak{g l}(l)^{\wedge}$-module structure to this module by

$$
\sigma_{c y c}^{\#}|\lambda, s ; r\rangle:=\left|\sigma_{c y c}^{\#}(\lambda, s ; r)\right\rangle \text {. }
$$

Definition $1.3 .4 \mathrm{c}\left(\mathbb{Z}^{l} \ltimes \mathfrak{g l}(l)^{\wedge}\right.$-modules).

(i) We define an action $t$ of $\mathbb{Z}^{l}$ on $\operatorname{gl}(l)^{\wedge}$ as follows.

$$
\begin{gathered}
t: \mathbb{Z}^{l}=\bigoplus_{k=1}^{l} \mathbb{Z}_{\varepsilon_{k}} \rightarrow \text { Aut }_{\text {Lie }} \mathrm{gl}(l)^{\wedge} \quad \text { (group hom), } \\
\left\{\begin{array}{l}
t_{\varepsilon_{k}}\left(E_{j}^{i}(n)\right):=E_{j}^{i}\left(n-\delta_{k i}+\delta_{k j}\right)-\delta_{n 0} \delta_{k i} \delta_{i j} \cdot c \\
t_{\varepsilon_{k}}(c):=c
\end{array}\right.
\end{gathered}
$$

(ii) A $\mathfrak{g l}(l)^{\wedge}$-module $(\pi, V)$ is called a $\mathbb{Z}^{l} \ltimes \mathfrak{g l}(l)^{\wedge}$-module if there exists some projective action $\pi^{\prime}$ of $\mathbb{Z}^{l}$ that satisfy $1.3 .2(1)$ for any $\sigma \in \mathbb{Z}^{l}$ and $g \in \operatorname{gl}(l)^{\wedge}$.

Sublemma 1.3.4. Let $V=(\pi, V)$ be a $\mathfrak{g l}(l)^{\wedge}$-module which is integrable as an $\mathfrak{s l}(l)^{\wedge}$ module. Then the following two conditions are equivalent:

(i) $V$ is a $\mathbb{Z}^{l} \ltimes \mathfrak{g l}(l)^{\wedge}$-module.

(ii) $V$ is $a\left\langle\sigma_{c y c}^{\#}\right\rangle \ltimes \mathfrak{g l}(l)^{\wedge}$-module.

Proof. By the integrability there is a projective action of the Weyl group $W^{\wedge} \simeq \mathfrak{S}_{l} \ltimes Q$ on $V$, where $Q \simeq\left\{\Sigma n_{j} \varepsilon_{j} \in \mathbb{Z}^{l} \mid \Sigma n_{j}=0\right\}$ denotes the root lattice of $A_{l-1}$ $([\mathrm{Kac}, \S 3])$. Let $r_{(1 \ldots l)}^{\pi} \in$ Aut $V$ be such an action of the cycle $(1, \ldots, l) \in \mathfrak{S}_{l}$. For (i) $\rightarrow$ (ii), the following formula gives the action of $\sigma_{c y c}^{\#}$ :

$$
\pi^{\prime}\left(\sigma_{c y c}^{\#}\right):=\pi^{\prime}\left(\varepsilon_{1}\right) r_{(1 \ldots l)}^{\pi} .
$$

As for the converse (ii) $\rightarrow$ (i), we can define the $\varepsilon_{1}$-action by

$$
\pi^{\prime}\left(\varepsilon_{1}\right):=\pi^{\prime}\left(\sigma_{c y c}^{\#}\right)\left(r^{\pi}{ }_{(1 \ldots l)}\right)^{-1} .
$$

This induces a $\mathbb{Z}^{l} \ltimes \mathfrak{g l}(l)^{\wedge}$-module structure on $V$, since there already exists a $Q$-action.

Put $\mathbb{1}^{*}:=\sum_{j=1}^{l} \varepsilon_{j} \in \mathbb{Z}^{l} \subset(\mathbb{C} 1)^{*}$ and define its action on $\mathbb{C 1}^{\wedge}$ by the restriction of (8), and let $\mathbb{Z}^{*} \times \mathbb{C}^{\wedge}$ be the corresponding Heisenberg system (1.3.3(1)).

Lemma 1.3.4. (i) Suppose that $V$ is a $\mathbb{Z}^{l} \ltimes \mathfrak{g l}(l)^{\wedge}$-module which satisfies the

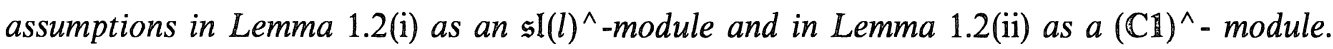
Then $V$ is completely reducible, i.e. decomposes into a direct sum of

$$
\mathbb{L}^{\mathbb{Z}^{l} \ltimes \mathrm{g} \mathrm{I}(l)^{\wedge}}(\lambda, s ; r):=\mathbb{L}^{\left\langle\sigma_{c y c}^{\#}\right\rangle \times \mathrm{g} \mathrm{I}(l)^{\wedge}}(\lambda, s ; r)^{\prime} s .
$$

(ii) If $r \in \mathbb{Z}_{>0}$ and $(\lambda ; r)$ is dominant integral as an $\mathfrak{s l}(l)^{\wedge}$-weight, then we have a $\left(\mathbb{Z} \mathbb{1}^{*} \propto \mathbb{C l}^{\wedge}\right) \oplus \mathfrak{s l}(l)^{\wedge}$-module isomorphism

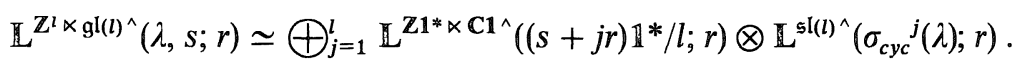

Proof. (i) This follows from a similar consideration as in the proof of Lemma 1.3.2 with using Sublemma 1.3.4. Regard $V$ as a $\left\langle\sigma_{c y c}^{\#}\right\rangle \ltimes \operatorname{gl}(l)^{\wedge}$-module and consider the $\left\langle\sigma_{c y c}^{\#}\right\rangle$-orbit of a highest weight vector with respect to $\operatorname{gl}(l)^{\wedge}$.

(iii) Consider the $\sigma_{c y c}^{\#}$-action in the left hand side of (12). It acts on the $s \mathfrak{l}(l)^{\wedge}$ components as an $\sigma_{c y c}$ and the action of $\left(\sigma_{c y c}^{\#}\right)^{l}$ coinsides with that of $\mathbb{1}^{*}$ on the whole 
module. Hence recalling the representation theory of the Heisenberg system [Lemma 1.2(ii); FK, Proposition 2.4] we have

$$
\begin{aligned}
& \mathbb{L}^{Z^{l} \ltimes \mathrm{gl}(l) \wedge}(\lambda, s ; r)=\mathbb{L}^{\left\langle\sigma_{c y c}^{\#}\right\rangle \ltimes \mathrm{gl}(l) \wedge}(\lambda, s ; r) \\
& =\bigoplus_{j=1}^{l} \bigoplus_{n \in \mathbf{Z}} \mathbf{L}^{\mathrm{gl}(l)^{\wedge}}\left(\left(\sigma_{c y c}^{\#}\right)^{j+l n} \cdot(\lambda, s ; r)\right) \\
& =\bigoplus_{j=1}^{l} \bigoplus_{n \in \mathbf{Z}} \mathbb{L}^{\mathrm{gl}(l)^{\wedge}}\left(\left(\sigma_{c y c}\right)^{j}(\lambda), s+(j+\ln ) r ; r\right) \\
& \simeq \bigoplus_{j=1}^{l} \mathbb{L}^{\mathbf{Z 1} 1^{*} \times \mathbb{C} 1^{\wedge}}\left((s+j r) \mathbb{1}^{*} / l ; r\right) \otimes \mathbb{L}^{\mathfrak{s}(l) \wedge}\left(\sigma_{c y c}^{j}(\lambda) ; r\right) \text {. }
\end{aligned}
$$

In view of 1.3.3(5), the statement of (ii) above can be interpreted in terms of characters as follows. Put

$$
\chi_{\mathrm{Z}^{l} \ltimes \mathrm{gl}(l)^{\wedge}}^{\lambda, s ; r}:=\operatorname{ch} \mathbb{L}^{\mathbf{Z}^{l} \ltimes \mathrm{gl}(l)^{\wedge}}(\lambda, s ; r)
$$

(1.2.3(6)). Then we have

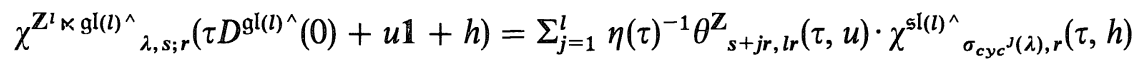

for $h \in\left\{\Sigma a_{i} E_{i}^{i} \mid \Sigma a_{i}=0\right\}, u, \tau \in \mathbb{C}$ and $\operatorname{Im} \tau>0$, where

$$
\theta_{v, m}^{\mathbf{Z}}(\tau, u):=\Sigma_{\mu \in v+m \mathbb{Z}} \mathbf{e}\left[\mu u+\mu^{2} \tau / 2 m\right]
$$

(1.5.1(0)) and $\eta$ denotes the Dedekind eta function (1.5.1(14)).

Remark 1.3.4. The character (14) is essentially introduced by Jimbo and Miwa first. See [JM, p. 34] for its further formulae.

\subsection{Notations for classical simple Lie algebras}

In 1.4.1 we give some definitions on Young diagrams.

We give the following data in 1.4.2 1.4.5.

(0) Dynkin diagram;

(1.1) Realization, (1.2) the Chevalley generators, (1.3) commutation relations;

(2.1) a Cartan subalgebra, (2.2) Simple roots, (2.3) positive roots and the root spaces, (2.4) the highest root;

(3.1) Fundamental weights, (3.2) half sum of positive roots, (3.3) dominant integral weights and their parametrization via Young diagrams;

(4) Character formula in terms of determinant;

(5.1) Normalized invariant symmetric form and (5.2) the induced bilinear form on $\mathfrak{h}^{*}$.

\subsubsection{Young diagrams}

A sequence of nonnegative integers $Y=\left(y_{j}\right)_{j=1}^{l}$ with $y_{1} \geq \cdots \geq y_{l} \geq 0$ is called a Young diagram. The set of all such $Y$ 's are denoted by $\mathscr{Y}_{l}$. We put $\mathscr{Y}_{l, r}:=$ $\left\{\left(y_{j}\right) \in \mathscr{Y}_{l} \mid r \geq y_{1}\right\}$ and call its element a Young diagram contained in the $l \times r$ rectangle. The size of $Y=\left(y_{j}\right)$ is $[Y]:=\Sigma_{j} y_{j} \in \mathbf{Z}_{\geq 0}$. For $Y=\left(y_{j}\right) \in \mathscr{Y}_{l, r}$, its complement (in $\mathscr{Y}_{l, r}$ ) $Y^{c}=\left(y_{j}^{c}\right)_{j=1}^{r} \in \mathscr{Y}_{l, r}$ is defined by $y_{j}^{c}:=n-y_{m-j}$. The transposed of $Y$ is denoted by ${ }^{t} Y$; it 
is defined by folding along the diagonal in the graphical expression and belongs to $\mathscr{Y}_{r, l}$. Also put $Y^{\dagger}=\left(y_{p}^{\dagger}\right)_{p=1}^{r}:=\left({ }^{t} Y\right)^{c}={ }^{t}\left(Y^{c}\right) \in \mathscr{Y}_{r, l}$ for $Y \in \mathscr{Y}_{l, r}$. For example,

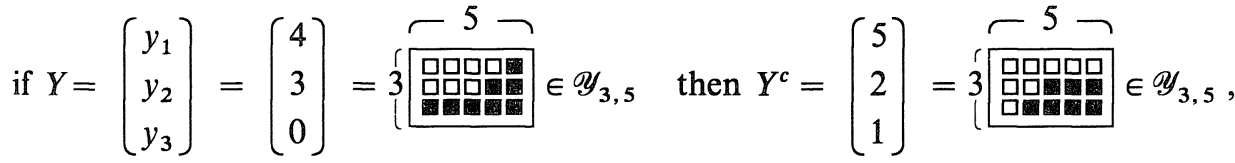

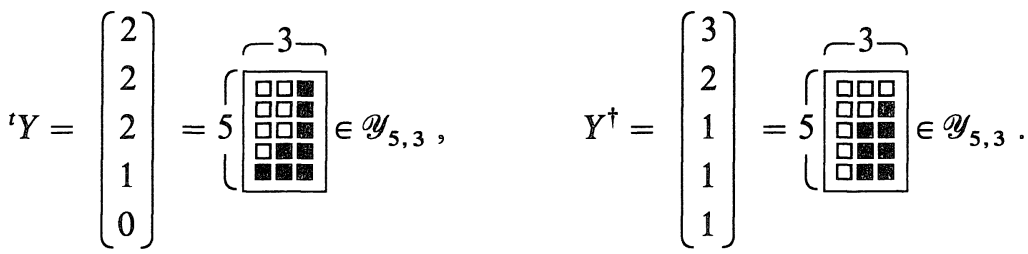

1.4.2. Type $A_{r-1}$

(0)

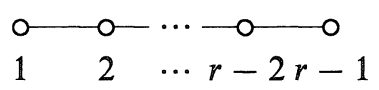

(1.1) $\mathfrak{g}\left(A_{r-1}\right) \simeq \mathfrak{s l}(r):=\{x \in \mathfrak{g l}(r) \mid \operatorname{tr} x=0\}$

(1.2) $\quad e_{j} \leftrightarrow E_{j+1}^{j}$

$$
f_{j} \leftrightarrow E^{j+1}{ }_{j}
$$

$$
h_{j} \leftrightarrow E_{j}^{j}-E_{j+1}^{j+1} \quad(1 \leq j \leq r-1) .
$$

(1.3) $\left[E^{i}, E^{k}{ }_{l}\right]=\delta_{j}^{k} E_{l}^{i}-\delta_{l}{ }^{i} E^{k}{ }_{j}$.

(2.1) $\mathfrak{h}=\Sigma \mathbb{C}\left(E_{i}^{i}-E_{j}^{j}\right)=\left\{\Sigma u_{j} E_{j}^{j} \mid \Sigma u_{j}=0\right\} \subset \mathfrak{a}:=\bigoplus_{j=1}^{r} \mathbb{C} E_{j}^{j}$.

(2.2) $\alpha_{j}=\bar{\varepsilon}_{j}-\bar{\varepsilon}_{j+1}$, where $\left\{\varepsilon_{j}\right\}_{j=1}^{r} \subset \mathfrak{a}^{*}$ is the dual basis to $\left\{E_{j}^{j}\right\}_{j=1}^{r}$ and $\bar{\varepsilon}_{j}:=\varepsilon_{j}-r^{-1} \Sigma_{1}^{r} \varepsilon_{j}$.

(2.3) $\Delta_{+}=\left\{\bar{\varepsilon}_{i}-\bar{\varepsilon}_{j} \mid i<j\right\} . \quad \mathrm{g}_{\bar{\varepsilon}_{i}-\bar{\varepsilon}_{j}}=\mathbb{C} E_{j}^{i}$.

(2.4) $\theta=\bar{\varepsilon}_{1}-\bar{\varepsilon}_{r}$.

(3.1) $\Lambda_{j}=\bar{\varepsilon}_{1}+\cdots+\bar{\varepsilon}_{j} \quad(1 \leq j \leq r-1)$.

(3.2) $\rho=(r-1) \bar{\varepsilon}_{1}+\cdots+2 \bar{\varepsilon}_{r-2}+\bar{\varepsilon}_{r-1}$.

(3.3) $\mathscr{Y}_{r-1} \ni Y=\left(y_{j}\right)_{j=1}^{r-1} \mapsto \Sigma_{j=1}^{r-1} y_{j} \bar{\varepsilon}_{j} \in P_{+}$is bijective .

(3.3') $\mathscr{Y}_{r} \ni Y=\left(y_{j}\right)_{j=1}^{r} \mapsto \Sigma_{j=1}^{r} y_{j} \bar{\varepsilon}_{j} \in P_{+}$is surjective. The image of $Y$ will be also written by $Y$.

(4) For $Y \in P_{+}$and $z_{j} \in \mathbf{C}(1 \leq j \leq r)$ such that $\prod_{j} z_{j}=1$,

$$
\operatorname{Tr}_{\mathbf{L}(\mathbf{Y})}\left(\prod_{j} z_{j}^{E^{j}}\right)=\frac{\operatorname{det}\left[z_{j}^{r-i+y_{i}}\right]_{1 \leq i, j \leq r}}{\prod_{1 \leq i<j \leq r}\left(z_{i}-z_{j}\right)}
$$


(5.1) $\quad B(a, b)=\operatorname{Tr}_{\mathbb{C}^{r}}(a b) \quad$ for $a, b \in \mathfrak{s l}(r)$,

(5.2) $B(\alpha, \beta)=(\alpha \mid \beta)$ for $\alpha, \beta \in \mathfrak{h}^{*} \simeq\left\{\Sigma a_{j} \varepsilon_{j} \in \mathfrak{a}^{*} \mid \Sigma a_{j}=0\right\}$, where $\left(\varepsilon_{i} \mid \varepsilon_{j}\right):=\delta_{i j}$.

\subsubsection{Type $B_{r}$}

(0)

$$
\begin{aligned}
& 0 \quad 0-0-\cdots \rightarrow 0 \Rightarrow 0 \\
& 123 \cdots r-1 r
\end{aligned}
$$

(1.1) $\mathfrak{g}\left(B_{r}\right) \simeq \mathfrak{o}(2 r+1):=\left\{\left.x \in \mathfrak{g l}(2 r+1)\right|^{t} x J_{\mathfrak{v}}+J_{\mathfrak{v}} x=0\right\}$, where

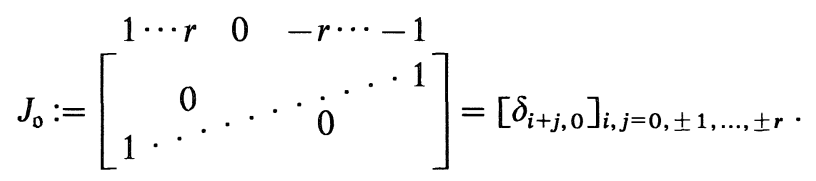

(1.2) Putting $B_{j}^{i}=B^{i,-j}=B_{-i, j}:=E_{j}^{i}-E_{-i}^{-j}$, the isomorphism is given by

$e_{j} \leftrightarrow B^{j+1}$ $\sqrt{2} B_{0}^{r}$

$f_{j} \leftrightarrow B^{j+1}{ }_{j}$

$(: 1 \leq j<r) ;$

$\sqrt{2} B^{0}$ $(: j=r)$

$h_{j} \leftrightarrow B_{j}^{j}-B_{j+1}^{j+1}$

$2 B^{r}$

(1.3) $\left[B^{i}{ }_{j}, B^{k}{ }_{l}\right]=\delta_{j}^{k} B_{l}^{i}-\delta^{i}{ }_{l} B_{j}^{k}+\delta^{-j}{ }_{l} B^{i}{ }_{-k}+\delta^{i}{ }_{-k} B^{-j}{ }_{l}$.

(2.1) $\mathfrak{h}=\bigoplus_{j=1}^{r} \mathbf{C} B^{j}{ }_{j}$.

(2.2)

$\alpha_{j}=\varepsilon_{j}-\varepsilon_{j+1} \quad(1 \leq j<r), \quad \alpha_{r}=\varepsilon_{r}$,

where $\left\{\varepsilon_{j}\right\}_{j=1}^{r} \subset \mathfrak{h}^{*}$ is the dual basis to $\left\{B^{j}\right\}_{j=1}^{r}$.

(2.3)

$\Delta_{+}=\left\{\varepsilon_{i} \pm \varepsilon_{j} \mid 1 \leq i<j \leq r\right\} \cup\left\{\varepsilon_{j} \mid 1 \leq j \leq r\right\}$

$$
\mathfrak{g}_{\varepsilon_{i} \pm \varepsilon_{j}}=\mathbb{C} B_{\mp j}^{i}, \quad \mathfrak{g}_{\varepsilon_{j}}=\mathbf{C} B^{j}{ }_{0} .
$$

(2.4) $\theta=\varepsilon_{1}+\varepsilon_{2}$.

(3.1) $\Lambda_{j}=\varepsilon_{1}+\cdots+\varepsilon_{j} \quad(1 \leq j<r), \quad \Lambda_{r}=\frac{1}{2}\left(\varepsilon_{1}+\cdots+\varepsilon_{r}\right)$.

(3.2) $\rho=\left(r-\frac{1}{2}\right) \varepsilon_{1}+\cdots+\frac{3}{2} \varepsilon_{r-1}+\frac{1}{2} \varepsilon_{r}$.

(3.3) $\quad P_{+}=P_{+}^{0} \coprod P_{+}^{1}$, where

$$
\begin{gathered}
P_{+}^{0}:=\Sigma_{j=1}^{r-1} \mathbb{Z}_{\geq 0} \Lambda_{j}+2 Z_{\geq 0} \Lambda_{r} \quad \text { and } \quad P_{+}^{1}:=P_{+}^{0}+\Lambda_{r} . \\
\mathscr{Y}_{r} \ni Y=\left(y_{j}\right) \mapsto \Sigma y_{j} \varepsilon_{j} \in P_{+}^{0}
\end{gathered}
$$

is bijective. The image of $Y$ will be also written by $Y$.

(4) $\operatorname{Tr}_{\mathbf{L}\left(Y+s \Lambda_{r}\right)}\left(\prod_{j} z_{j}^{B^{J} J}\right)=\frac{\operatorname{det}\left[z_{i}^{y_{j}+r-j+(s+1) / 2}-z_{i}^{-y_{j}-r+j-(s+1) / 2}\right]_{1 \leq i, j \leq r}}{\prod_{1 \leq j \leq r}\left(z_{j}^{1 / 2}-z_{j}^{-1 / 2}\right) \prod_{1 \leq i<j \leq r}\left(z_{i}-z_{j}\right)\left(1-z_{i}^{-1} z_{j}^{-1}\right)}$,

for $Y \in P_{+}^{0}, s=0,1$ and $z_{j} \in \mathbf{C}(1 \leq j \leq r)$. 
(5.1) $B(a, b)=\frac{1}{2} \operatorname{Tr}_{\mathbf{C}^{2 r+1}}(a b) \quad$ for $\quad a, b \in \mathfrak{o}(2 r+1)$.

(5.2) $B\left(\varepsilon_{i}, \varepsilon_{j}\right)=\left(\varepsilon_{i} \mid \varepsilon_{j}\right):=\delta_{i j}$.

\subsubsection{Type $\mathbb{C}_{r}$}

(0)

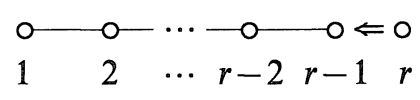

(1.1) $\mathfrak{g}\left(C_{r}\right) \simeq \mathfrak{s p}(2 r):=\left\{\left.x \in \mathfrak{g l}(2 r)\right|^{t} x J_{\mathfrak{s p}}+J_{\mathfrak{s p}} x=0\right\}$, where

$$
J_{\mathfrak{s p}}:=\left[\begin{array}{cc}
1 \cdots r & -r \cdots-1 \\
0 & \dot{ }^{-1} \\
1 & -1 \\
\therefore & 0
\end{array}\right]=\left[\operatorname{sgn}(j) \delta_{i+j, 0}\right]_{i, j= \pm 1, \ldots, \pm r}
$$

(1.2) Putting $C_{j}^{i}=C^{i,-j}=C_{-i, j}:=E_{j}^{i}-\operatorname{sgn}(i j) E_{-i}^{-j}$, the isomorphism is given by $e_{j} \leftrightarrow C^{j+1}$ $\frac{1}{2} C^{r}$

$f_{j} \leftrightarrow C_{j}^{j+1} \quad(: 1 \leq j<r) ; \quad \frac{1}{2} C_{r}^{-r} \quad(: j=r)$.

$h_{j} \leftrightarrow C^{j}{ }_{j}-C^{j+1}{ }_{j+1} \quad C^{r}{ }_{r}$

(1.3) $\left[C^{i}{ }_{j}, C^{k}{ }_{l}\right]=\delta_{j}^{k} C^{i}{ }_{l}-\delta^{i}{ }_{l} C^{k}{ }_{j}+(\operatorname{sgn} j k)\left(\delta^{-j}{ }_{l} C^{i}{ }_{-k}+\delta^{i}{ }_{-k} C^{-j}{ }_{l}\right)$.

(2.1) $\mathfrak{h}=\bigoplus_{j=1}^{r} \mathrm{CC}_{j}^{j}$.

(2.2) $\alpha_{j}=\varepsilon_{j}-\varepsilon_{j+1} \quad(1 \leq j<r), \quad \alpha_{r}=2 \varepsilon_{r}$, where $\left\{\varepsilon_{j}\right\}_{j=1}^{r} \subset \mathfrak{h}^{*}$ is the dual basis to $\left\{C^{j}{ }_{j}\right\}_{j=1}^{r}$.

(2.3) $\Delta_{+}=\left\{\varepsilon_{i} \pm \varepsilon_{j} \mid 1 \leq i<j \leq r\right\} \cup\left\{2 \varepsilon_{j} \mid 1 \leq j \leq r\right\}$ ，

$$
\mathfrak{g}_{\varepsilon_{i} \pm \varepsilon_{j}}=\mathbb{C} C_{\mp j}^{i}, \quad \mathfrak{g}_{2 \varepsilon_{j}}=\mathbf{C} C_{-j}^{j}
$$

(2.4) $\theta=2 \varepsilon_{1}$.

(3.1) $\Lambda_{j}=\varepsilon_{1}+\cdots+\varepsilon_{j} \quad(1 \leq j<r)$.

(3.2) $\rho=r \varepsilon_{1}+\cdots+2 \varepsilon_{r-1}+\varepsilon_{r}$.

(3.3) $\mathscr{Y}_{r} \ni Y=\left(y_{j}\right) \mapsto \Sigma y_{j} \varepsilon_{j} \in P_{+}$

is bijective. The image of $Y$ will be also written by $Y$.

(4) For $Y \in P_{+}$and $z_{j} \in \mathbb{C} \quad(1 \leq j \leq r)$,

$$
\operatorname{Tr}_{\mathbf{L}(Y)}\left(\prod_{j} z_{j}^{C^{j_{j}}}\right)=\frac{\operatorname{det}\left[z_{i}^{y_{j}+r+1-j}-z_{i}^{-\left(y_{j}+r+1-j\right)}\right]_{1 \leq i, j \leq r}}{\prod_{1 \leq j \leq r}\left(z_{j}-z_{j}^{-1}\right) \prod_{1 \leq i<j \leq r}\left(z_{i}-z_{j}\right)\left(1-z_{i}^{-1} z_{j}^{-1}\right)} .
$$


(5.1) $B(a, b)=\operatorname{Tr}_{\mathbf{C}^{2 r}}(a b) \quad$ for $\quad a, b \in \mathfrak{s p}(2 r)$.

(5.2) $B\left(\varepsilon_{i}, \varepsilon_{j}\right)=\left(\varepsilon_{i} \mid \varepsilon_{j}\right):=\frac{1}{2} \delta_{i j}$.

1.4.5. Type $\mathrm{D}_{r}$

(0)

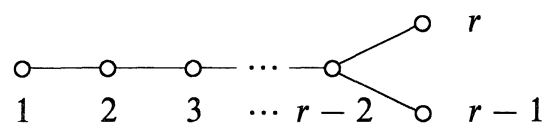

(1.1) $\mathfrak{g}\left(D_{r}\right) \simeq \mathfrak{v}(2 r):=\left\{\left.x \in \mathfrak{g l}(2 r)\right|^{t} x J_{\mathfrak{v}}+J_{\mathfrak{v}} x=0\right\}$, where

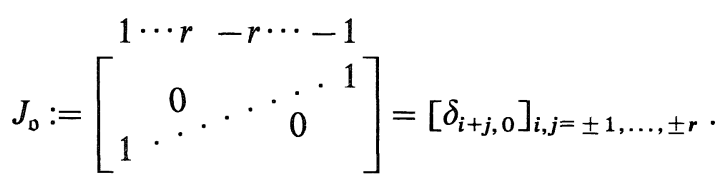

Putting $D_{j}^{i}=D^{i,-j}=D_{-i, j}:=E_{j}^{i}-E_{-i}^{-j}$, the isomorphism is given by

$$
\begin{array}{lll}
e_{j} \leftrightarrow D_{j+1}^{j}{ }_{j+1} & D^{r-1, r} \\
f_{j} \leftrightarrow D_{j}^{j+1}{ }_{j} & (: 1 \leq j \leq r) ; & D_{r-1, r} \quad(: j=r) . \\
h_{j} \leftrightarrow D_{j}^{j}{ }_{j}-D_{j+1}^{j+1}{ }_{j+1} & & D^{r-1}{ }_{r-1}-D_{r}^{r}
\end{array}
$$

$\left[D_{j}^{i}, D^{k}{ }_{l}\right]=\delta_{j}^{k} D^{i}{ }_{l}-\delta^{i}{ }_{l} D^{k}{ }_{j}+\delta^{-j}{ }_{l} D^{i}{ }_{-k}+\delta^{i}{ }_{-k} D^{-j}{ }_{l}$.

(2.1) $\mathfrak{h}=\bigoplus_{j=1}^{r} \mathbf{C} D_{j}^{j}$

(2.2) $\alpha_{i}=\varepsilon_{j}-\varepsilon_{j+1} \quad(1 \leq j<r), \quad \alpha_{r}=\varepsilon_{r-1}+\varepsilon_{r}$,

where $\left\{\varepsilon_{j}\right\}_{j=1}^{r} \subset \mathfrak{h}^{*}$ is the dual basis to $\left\{D^{j}\right\}_{j=1}^{r}$.

(2.3) $\Delta_{+}=\left\{\varepsilon_{i} \pm \varepsilon_{j} \mid 1 \leq i<j \leq r\right\}, \quad \mathfrak{g}_{\varepsilon_{i} \pm \varepsilon_{j}}=\mathbf{C} D_{ \pm j}^{i}$.

(2.4) $\theta=\varepsilon_{1}+\varepsilon_{2}$.

(3.1) $\Lambda_{j}=\varepsilon_{1}+\cdots+\varepsilon_{j} \quad(1 \leq j \leq r-2)$,

$$
\Lambda_{r-1}=\frac{1}{2}\left(\varepsilon_{1}+\cdots+\varepsilon_{r-1}-\varepsilon_{r}\right), \quad \Lambda_{r}=\frac{1}{2}\left(\varepsilon_{1}+\cdots+\varepsilon_{r-1}+\varepsilon_{r}\right) .
$$

(3.2) $\rho=(r-1) \varepsilon_{1}+\cdots+2 \varepsilon_{r-2}+\varepsilon_{r-1}$.

(3.3) $P_{+}=\left(P_{+}^{0} \cup \sigma\left(P_{+}^{0}\right)\right) \coprod P_{+}^{1} \coprod \sigma\left(P_{+}^{1}\right) \quad$ (disjoint union).

Here $\quad P_{+}^{0}:=\left\{\lambda=\Sigma \lambda_{j} \varepsilon_{j} \in P_{+} \mid \forall \lambda_{j} \in \mathbb{Z}_{\geq 0}\right\}, \quad P_{+}^{1}:=P_{+}^{0}+\Lambda_{r}, \quad$ and $\quad \sigma: \mathfrak{h}^{*} \ni \varepsilon_{j} \mapsto$ $\left(1-2 \delta_{j r}\right) \varepsilon_{j} \in \mathfrak{h}^{*}$ is the action of the diagram automorphism $\sigma_{r-1, r}:=\sigma_{(r-1, r)}$. The map

$$
\mathscr{Y}_{r} \ni Y=\left(y_{j}\right) \mapsto \Sigma y_{j} \varepsilon_{j} \in P_{+}^{0}
$$

is bijective. The image of $Y$ will be also written by $Y$. 
(4) For $\lambda=\sum \lambda_{j} \varepsilon_{j} \in P_{+}$and $z_{j} \in \mathbb{C}(1 \leq j \leq r)$,

$$
\begin{gathered}
\operatorname{Tr}_{\mathbb{L}(\lambda)}\left(\prod_{j} z_{j}^{D^{J_{j}}}\right)=\sum_{\left(s_{j}\right) \in\{ \pm 1\}{ }^{r}, \prod_{1}^{r} s_{j}=1} \frac{\operatorname{det}\left[z_{i}^{s_{j}\left(\lambda_{j}+r-j\right)}\right]_{1 \leq i, j \leq r}}{\prod_{1 \leq i<j \leq r}\left(z_{i}-z_{j}\right)\left(1-z_{i}^{-1} z_{j}^{-1}\right)}, \\
\operatorname{Tr}_{\mathbb{L}(\lambda)}\left(\prod_{j} z_{j}^{D J_{j}}\right) \pm \operatorname{Tr}_{\mathbb{L}(\sigma(\lambda))}\left(\prod_{j} z_{j}^{D^{j}}\right)=\frac{\operatorname{det}\left[z_{i}^{\lambda_{j}+r-j} \pm z_{i}^{-\lambda_{j}-r+j}\right]_{1 \leq i, j \leq r}}{\prod_{1 \leq i<j \leq r}\left(z_{i}-z_{j}\right)\left(1-z_{i}^{-1} z_{j}^{-1}\right)} .
\end{gathered}
$$

(5.1) $B(a, b)=\frac{1}{2} \operatorname{Tr}_{\mathbb{C}^{2 r}}(a b) \quad$ for $\quad a, b \in \mathfrak{o}(2 r)$.

(5.2) $B\left(\varepsilon_{i}, \varepsilon_{j}\right)=\left(\varepsilon_{i} \mid \varepsilon_{j}\right):=\delta_{i j}$.

\subsubsection{A convention for $\mathfrak{o}(n)$}

Put $\mathfrak{o}\left(\mathbb{1}_{N}\right):=\left\{X \in \operatorname{gl}(N) \mid X+{ }^{t} X=0\right\}$ and

$$
O_{j}^{i}:=E_{j}^{i}-E_{i}^{j} \in \mathfrak{o}\left(\mathbb{H}_{N}\right) .
$$

Here the indeces $i, j$ run over $-n,-n+1, \ldots, n-1, n$, where $n:=\left[\frac{N}{2}\right]$ (we assume that $i, j$ do not take zero if $N$ is even). Then the isomorphism $\mathfrak{o}\left(\mathbb{1}_{N}\right) \simeq \mathfrak{o}(N)$ can be given as follows.

(i) Case of $N=2 n+1$. For $i, j>0$,

$$
\begin{aligned}
& O_{-j}^{i} \leftrightarrow \frac{\sqrt{-1}}{2}\left(B_{j}^{i}+B_{i}^{j}-B^{i j}+B_{i j}\right) . \\
& O_{j}^{i} \leftrightarrow \frac{1}{2}\left(B_{j}^{i}-B_{i}^{j}+B^{i j}+B_{i j}\right), \\
& O^{-i}{ }_{-j} \leftrightarrow \frac{1}{2}\left(B_{j}^{i}-B_{i}^{j}-B^{i j}-B_{i j}\right), \\
& O_{ \pm j}^{0} \leftrightarrow \frac{1}{\sqrt{ \pm 2}}\left(B^{0}{ }_{j} \pm B^{0}{ }_{-j}\right) .
\end{aligned}
$$

(ii) Case of $N=2 n$. For $i, j>0$,

$$
\begin{aligned}
& O_{-j}^{i} \leftrightarrow \frac{\sqrt{-1}}{2}\left(D_{j}^{i}+D^{j}{ }_{i}-D^{i j}+D_{i j}\right), \\
& O_{j}^{i} \leftrightarrow \frac{1}{2}\left(D_{j}^{i}-D^{j}{ }_{i}+D^{i j}+D_{i j}\right), \\
& O^{-i}{ }_{-j} \leftrightarrow \frac{1}{2}\left(D_{j}^{i}-D^{j}{ }_{i}-D^{i j}-D_{i j}\right) .
\end{aligned}
$$

Then we introduce and fix a root basis of $\mathfrak{o}\left(\mathbb{1}_{N}\right)$ by this isomorphism. We also denote them by $\left\{B_{j}^{i}\right\}$ or $\left\{D_{j}^{i}\right\}$, respectively. 


\subsection{Notations for classical affine Lie algebras}

In 1.5.1 we summarize some theta functions and their formulae.

In $1.5 .2 \sim 1.5 .5$, following the notation in 1.4 we give

(0) Dynkin diagram (of classical nontwisted affine type), the dual label $\check{a}_{i}$ and the dual Coxeter number $g$;

(1) parametrization of the dominant integral weights $\bmod \mathbb{C} \delta$;

(2) Weyl group $W \ltimes M$;

(3) the numerator $\mathscr{A}_{\lambda, l}$ and the denominator $\mathscr{D}(1.2 .3(9))$; and

(4) actions of the diagram automorphisms defined in 1.3.1.

For the sake of completeness, we list the rest of affine Dynkin diagrams and their dual labels in 1.5.6.

\subsubsection{Theta functions}

Let $l \in \mathbf{R}_{>0}, j \in \mathbf{R}, \tau \in \mathbf{C}_{+}:=\{\tau \in \mathbb{C}, \operatorname{Im} \tau>0\}, u \in \mathbf{C}$ and put $\mathbf{e}[*]:=\exp (2 \pi i *)$, $i:=\sqrt{-1}$. We define

$$
\theta_{j, l}^{\mathbf{Z}, \pm}(\tau, u):=\Sigma_{n \in \mathbb{Z}}( \pm 1)^{n} \mathbf{e}\left[(j+\ln ) u+\frac{(j+\ln )^{2}}{2 l} \tau\right]
$$

Then $\theta^{\mathbf{Z},+}{ }_{j, l}=\theta_{j, l}^{\mathbf{Z}}$ and $\theta^{\mathbf{Z},-}{ }_{j, l}(\tau, u)=\mathbf{e}[-j / 2 l] \theta_{j, l}^{\mathbf{Z}}(\tau, u+1 / 2 l)$, where $\theta_{j, l}^{\mathbf{Z}}$ is as in 1.3.4(15). We have the following formulae.

$$
\theta_{j+l, l}^{\mathbf{Z}_{j, l}}=\theta_{j, l}^{\mathbf{Z}}, \quad \theta_{j, l}^{\mathbf{Z}_{j}}(\tau,-u)=\theta_{-j, l}^{\mathbf{Z}}(\tau, u), \quad \theta_{j, l}^{\mathbf{Z}}(\tau, u)=\theta_{j / l, 1}^{\mathbf{Z}_{j, 1}}(l \tau, l u)
$$

(2) $\theta_{j, l}^{\mathbf{Z}}(\tau, u) \theta_{k, m}^{\mathbf{Z}}(\tau, v)$

$$
=\Sigma_{\substack{\mu \in \mathbf{R} \bmod l(l+m) \\ \mu \equiv j+k \bmod l}} \theta_{\mu, l+m}^{\mathbf{Z}}\left(\tau, \frac{l u+m v}{l+m}\right) \theta_{m \mu-(l+m) k, l m(l+m)}^{\mathbf{Z}}\left(\tau, \frac{u-v}{l+m}\right) \quad\left(l, m \in \mathbb{Z}_{>0}\right)
$$

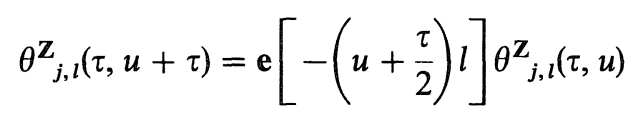

$$
\begin{aligned}
& \theta_{j, l}^{\mathbf{z}_{j}}\left(\tau, u \pm \frac{\tau}{2}\right)=\mathbf{e}\left[-\left( \pm \frac{u}{2}+\frac{\tau}{8}\right) l\right] \theta_{j \pm l / 2, l}^{\mathbf{z}}(\tau, u) \\
& \theta_{j, l}^{\mathbf{Z}}(\tau, u+1)=\mathbf{e}[j] \theta_{j, l}^{\mathbf{Z}}(\tau, u) \quad(\text { if } l \in \mathbb{Z})
\end{aligned}
$$

For $j, l \in \mathbb{Z}$, we also have

$$
\begin{aligned}
& \theta_{j, l}^{\mathbf{Z}}(\tau+1, u)=\mathbf{e}[j(j-l) / 2 l] \theta^{\mathbb{Z}}{ }_{j, l}\left(\tau, u+\frac{1}{2}\right), \\
& \theta_{j+l / 2, l}^{\mathbf{Z}}(\tau+1, u)=\mathbf{e}[1 / 8+j(j-l) / 2 l] \theta_{j, l}^{\mathbf{Z}}(\tau, u),
\end{aligned}
$$

and the following transformation rules by Poisson's summation formula [Se].

$$
\mathbf{e}\left[-\frac{l u^{2}}{2 \tau}\right] \theta_{j, l}^{\mathbf{Z}}\left(-\frac{1}{\tau}, \frac{u}{\tau}\right)=\sqrt{-i \tau} \Sigma_{k=1}^{l} \frac{\mathbf{e}[-j k / l]}{\sqrt{l}} \theta_{k, l}^{\mathbb{Z}}(\tau, u)
$$


(9)

$$
\begin{gathered}
\mathbb{e}\left[-\frac{l u^{2}}{2 \tau}\right] \theta^{\mathbb{Z}}{ }_{j+l / 2, l}\left(-\frac{1}{\tau}, \frac{u}{\tau}\right)=\sqrt{-i \tau} \Sigma_{k=1}^{l} \frac{\mathrm{e}[-j k / l]}{\sqrt{l}} \theta_{k, l}^{\mathbb{Z}}\left(\tau, u+\frac{1}{2}\right) \\
\mathbb{e}\left[-\frac{l u^{2}}{2 \tau}\right] \theta_{j, l}^{\mathbb{Z}}\left(-\frac{1}{\tau}, \frac{u}{\tau}+\frac{1}{2}\right)=\sqrt{-i \tau} \Sigma_{k=1}^{l} \frac{\mathrm{e}[-j k / l]}{\sqrt{l}} \theta^{\mathbb{Z}}{ }_{k+l / 2, l}(\tau, u) \\
\mathbb{e}\left[-\frac{l u^{2}}{2 \tau}\right] \theta^{\mathbb{Z}}{ }_{j+l / 2, l}\left(-\frac{1}{\tau}, \frac{u}{\tau}+\frac{1}{2}\right)=\sqrt{-i \tau}(-i)^{l} \sum_{k=1}^{l} \frac{e[-j k / l]}{\sqrt{l}} \theta_{k+l / 2, l}^{\mathbb{Z}}\left(\tau, u+\frac{1}{2}\right)
\end{gathered}
$$

Here we choose $\sqrt{-i \tau}$ such that $\operatorname{Re} \sqrt{-i \tau}>0$.

In the case of $l=1$, we use following notations after [JM].

$$
\begin{aligned}
\theta(\tau, u) & :=-i \theta_{1 / 2,1}^{\mathbb{Z}}\left(\tau, u+\frac{1}{2}\right) \\
& =\Sigma_{n \in \mathbb{Z}}(-1)^{n} \mathbb{e}\left[\frac{1}{2}\left(n+\frac{1}{2}\right)^{2} \tau+\left(n+\frac{1}{2}\right) u\right] \\
& =q^{1 / 8}\left(z^{1 / 2}-z^{-1 / 2}\right) \prod_{n \geq 1}\left(1-q^{n}\right)\left(1-z q^{n}\right)\left(1-z^{-1} q^{n}\right) \\
& =-\theta(\tau, u+1)=-\theta(\tau,-u)=-z q^{1 / 2} \theta(\tau, u+\tau) \\
& =\mathbb{e}\left[1 / 4-u^{2} / 2 \tau\right] \sqrt{-i \tau}-1 \theta(-1 / \tau, u / \tau), \\
\hat{\theta}(\tau, u) & :=\theta^{\mathbb{Z}}{ }_{0,1}\left(\tau, u+\frac{1}{2}\right) \\
& =\Sigma_{n \in \mathbb{Z}}(-1)^{n} \mathbb{e}\left[\frac{1}{2} n^{2} \tau+n u\right] \\
& =\prod_{n \geq 1}\left(1-q^{n}\right)\left(1-z q^{n-1 / 2}\right)\left(1-z^{-1} q^{n-1 / 2}\right) \\
& =\hat{\theta}(\tau, u+1)=\hat{\theta}(\tau,-u)=z^{-1 / 2} q^{1 / 8} \theta\left(\tau, u-\frac{\tau}{2}\right) \\
& =\mathbb{e}\left[-u^{2} / 2 \tau\right] \sqrt{-i \tau}-1 \hat{\theta}(-1 / \tau, u / \tau),
\end{aligned}
$$

where $q:=\mathbb{e}[\tau]$ and $z:=\mathbb{e}[u]$.

Recall the Dedekind eta function and its transformation rule,

$$
\eta(\tau):=q^{1 / 24} \prod_{n=1}^{\infty}\left(1-q^{n}\right)=\Sigma_{n \in \mathbb{Z}}(-1)^{n} q^{(6 n+1)^{2} / 24}=\sqrt{-i \tau}^{-1} \eta(-1 / \tau) .
$$

1.5.2. Type $A_{r-1}^{(1)}$

(0)

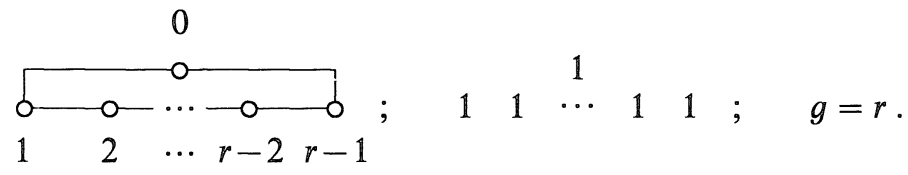

(1) There is a surjection

$$
\mathscr{Y}_{r, l} \ni Y \mapsto(Y ; l)=\left.Y\right|_{\mathfrak{h}}+l \hat{\Lambda}_{0} \in \hat{P}_{+}(l) .
$$


(2) $W \simeq \mathfrak{S}_{r}, \quad M=Q:=\left\{\Sigma m_{j} \varepsilon_{j} \in \bigoplus_{j=1}^{r} Z_{\varepsilon_{j}} \mid \Sigma_{j} m_{j}=0\right\} \subset \mathfrak{h}^{*}$.

(3) For an $r$-vector $u=\left(u_{j}\right)_{j=1}^{r}$, put $[u]:=\Sigma_{j=1}^{r} u_{j}$ and $\bar{u}_{j}:=u_{j}-[u] / r$. By $\Lambda_{k}$ $(0 \leq k \leq r-1)$ we denote the classical part of the (affine) fundamental weight $\hat{\Lambda}_{k}$ (1.4.1(3.1)). Then

$$
\sum_{k=0}^{r-1} \mathscr{A}_{l \Lambda_{k}+\Sigma_{j=1}^{r} \lambda_{j} \bar{\varepsilon}_{j}, l}\left(\tau, \Sigma_{j=1}^{r} \bar{u}_{j} E_{j}^{j}\right) \theta_{[\lambda]+l k, l r}^{\mathbf{Z}}\left(\tau, \frac{[u]}{r}\right)=\operatorname{det}\left[\theta_{\lambda_{\imath}, l}^{\mathbf{Z}}\left(\tau, u_{j}\right)\right]_{1 \leq i, j \leq r}
$$

for $\Sigma \lambda_{j} \varepsilon_{j} \in \mathfrak{o}^{*}(1.4 .1)$

$$
\mathscr{D}\left(\tau, \Sigma_{j=1}^{r} \bar{u}_{j} E_{j}^{j}\right)=\eta(\tau)^{-(r-1)(r-2) / 2} \prod_{1 \leq i<j \leq r} \theta\left(\tau, \bar{u}_{i}-\bar{u}_{j}\right)
$$

(4) Put $c y c:=(0,1, \ldots, r-1), w:=\left(\begin{array}{ccccc}0 & 1 & \ldots & r-2 & r-1 \\ 0 & r-1 & \ldots & 2 & 1\end{array}\right)$ and let $\sigma_{c y c}$ and $\sigma_{w}$ respectively be the corresponding diagram automorphisms. Then $\sigma_{w} \sigma_{c y c} \sigma_{w}{ }^{-1}=$ $\sigma_{c y c}{ }^{-1}$, and we have

$$
\begin{aligned}
& \sigma_{c y c}\left(s c+\tau d+\Sigma_{j=1}^{r} u_{j} E_{j}^{j}\right) \\
& \quad=\left(s-\tau(r-1) / 2 r-u_{r}\right) c+\tau d+\Sigma_{j=1}^{r}\left(u_{j-1}+\left(\delta_{j 1}-1 / r\right) \tau\right) E_{j}^{j}, \\
& \sigma_{c y c}\left(s \delta+l \hat{\Lambda}_{0}+\Sigma_{j=1}^{r} y_{j} \varepsilon_{j}\right) \\
& \quad=\left(s-l(r-1) / 2 r-y_{r}\right) \delta+l \hat{\Lambda}_{0}+\Sigma_{j=1}^{r}\left(y_{j-1}+\left(\delta_{j 1}-1 / r\right) l\right) \varepsilon_{j}
\end{aligned}
$$

and

$$
\begin{aligned}
& \sigma_{w}\left(s c+\tau d+\sum_{j=1}^{r} u_{j} E_{j}^{j}\right)=s c+\tau d+\Sigma_{j=1}^{r}\left(-u_{r+1-j}\right) E_{j}^{j}, \\
& \sigma_{w}\left(s \delta+l \hat{\Lambda}_{0}+\Sigma_{j=1}^{r} y_{j} \varepsilon_{j}\right)=s \delta+l \hat{\Lambda}_{0}+\Sigma_{j=1}^{r}\left(-y_{r+1-j}\right) \varepsilon_{j},
\end{aligned}
$$

where we assume $\Sigma_{j=1}^{r} u_{j}=0$ and $\Sigma_{j=1}^{r} y_{j}=0$.

cyc:
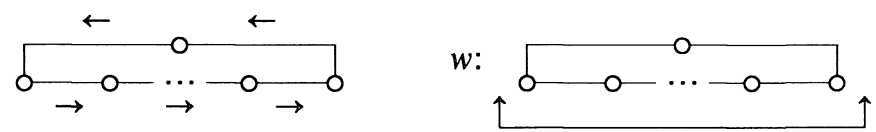

\subsubsection{Type $B_{r}^{(\mathbf{1})}$}

(0)

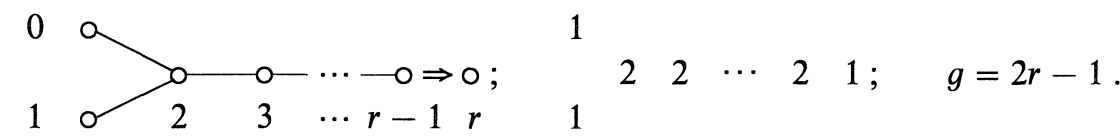

(1) $\hat{P}_{+}(L)=L \hat{\Lambda}_{0}+\left\{\begin{array}{l|l}\sum_{j=1}^{r} y_{j} \varepsilon_{j} & \begin{array}{l}\frac{L}{2} \geq \min \left(y_{1}, L-y_{1}\right) \geq y_{2} \geq \cdots \geq y_{r} \geq 0, \\ \left(y_{1}, \ldots, y_{r}\right) \in(\mathbf{Z}+s)^{r}, \quad s=0 \text { or } 1 / 2\end{array}\end{array}\right\}$

(2) $W \simeq \mathfrak{S}_{r} \ltimes\{ \pm 1\}^{r}, \quad M=\left\{\Sigma m_{j} \varepsilon_{j} \in \bigoplus_{j=1}^{r} \mathbb{Z} \varepsilon_{j} \mid \Sigma m_{j} \in 2 \mathbb{Z}\right\} \subset \mathfrak{h}^{*}$. 
(3) For $u=\sum_{j=1}^{r} u_{j} B_{j}^{j} \in \mathfrak{h}$ and $\lambda=\sum_{j=1}^{r} \lambda_{j} \varepsilon_{j} \in \mathfrak{h}^{*}$,

$$
\begin{aligned}
& \mathscr{A}_{\lambda+l \hat{\Lambda}_{0}}(\tau, u) \pm \mathscr{A}_{\sigma_{01}\left(\lambda+l \hat{\Lambda}_{0}\right)}(\tau, u)=\operatorname{det}\left[\theta_{\lambda_{i}, l}^{\mathbb{Z}, \mp}\left(\tau, u_{j}\right)-\theta_{-\lambda_{l}, l}^{\mathbb{Z}, \mp}\left(\tau, u_{j}\right)\right]_{1 \leq i, j \leq r}, \\
& \mathscr{D}(\tau, u)=\eta(\tau)^{-r(r-1)} \prod_{1 \leq i<j \leq r} \theta\left(\tau, u_{i}-u_{j}\right) \theta\left(\tau, u_{i}+u_{j}\right) \prod_{1 \leq j \leq r} \theta\left(\tau, u_{j}\right) .
\end{aligned}
$$

(4) The diagI am automorphism $\sigma_{0}=\sigma_{01}$ that corresponds to the permutation $(0,1)$ is given by

$$
\begin{aligned}
& \sigma_{01}\left(s c+\tau d+\Sigma_{j=1}^{r} u_{j} B^{j}{ }_{j}\right)=\left(s-\tau / 2+u_{1}\right) c+\tau d+\left(\tau-u_{1}\right) B^{1}{ }_{1}+\Sigma_{j=2}^{r} u_{j} B_{j}^{j}, \\
& \sigma_{01}\left(s \delta+L \hat{\Lambda}_{0}+\sum_{j=1}^{r} y_{j} \varepsilon_{j}\right)=\left(s-L / 2+y_{1}\right) \delta+L \hat{\Lambda}_{0}+\left(L-y_{1}\right) \varepsilon_{1}+\sum_{j=2}^{r} y_{j} \varepsilon_{j} . \\
& (0,1) \text { : } \\
& \Gamma_{0}^{a}+\cdots \longrightarrow 0
\end{aligned}
$$

It holds that $\sigma_{01}=t_{\varepsilon_{1}} \circ r_{\varepsilon_{1}} \in$ Aut $\tilde{\mathfrak{h}}^{*}$, where $t_{\alpha}$ (resp. $r_{\alpha}$ ) denotes the translation (resp. the reflection) associated to $\alpha$ : for $\Lambda \in \tilde{\mathfrak{h}}^{*}$,

$$
\begin{aligned}
& t_{\alpha}(\Lambda):=\Lambda+\Lambda(c) \cdot \alpha+\left\{B(\Lambda, \alpha)+\frac{1}{2} \Lambda(c) \cdot B(\alpha, \alpha)\right\} \delta \\
& r_{\alpha}(\Lambda):=\Lambda-2 \frac{B(\Lambda, \alpha)}{B(\alpha, \alpha)} \alpha .
\end{aligned}
$$

\subsubsection{Type $C_{r}^{(1)}$}

(0) $\circ \Rightarrow 0 \quad 0-\cdots-\circ \quad 0 \Leftarrow 0 ; \quad 1 \quad 1 \quad 1 \quad \cdots \quad 1 \quad 1 \quad 1 ; \quad g=r+1$.

$\begin{array}{lllllll}0 & 1 & 2 & \cdots & r-2 & r-1 & r\end{array}$

(1) There is a bijection

$$
\mathscr{Y}_{r, l} \ni Y \underset{\leftrightarrow}{\simeq}(Y ; l)=Y+l \hat{\Lambda}_{0} \in \hat{P}_{+}(l)
$$

(2) $W \simeq \mathfrak{S}_{r} \ltimes\{ \pm 1\}^{r}, \quad M=\bigoplus_{j=1}^{r} 2 \mathbb{Z} \varepsilon_{j} \subset \mathfrak{h}^{*}$.

(3) For $u=\sum_{j=1}^{r} u_{j} C^{j}{ }_{j} \in \mathfrak{h}$ and $\lambda=\sum_{j=1}^{r} \lambda_{j} \varepsilon_{j} \in \mathfrak{h}^{*}$,

$$
\begin{aligned}
\mathscr{A}_{\lambda, l}(\tau, u) & =\operatorname{det}\left[\theta^{\mathbb{Z}}{ }_{\lambda_{i}, l}\left(\tau, 2 u_{j}\right)-\theta^{\mathbb{Z}}{ }_{-\lambda_{i}, l}\left(\tau, 2 u_{j}\right)\right]_{1 \leq i, j \leq r}, \\
\mathscr{D}(\tau, u) & =\eta(\tau)^{-r(r-1)} \prod_{1 \leq i<j \leq r} \theta\left(\tau, u_{i}-u_{j}\right) \theta\left(\tau, u_{i}+u_{j}\right) \prod_{1 \leq j \leq r} \theta\left(\tau, 2 u_{j}\right) .
\end{aligned}
$$

(4) Put $w:=\left(\begin{array}{cccc}0 & \cdots & r-1 & r \\ r & \cdots & 1 & 0\end{array}\right)$ and let $\sigma_{w}$ be the corresponding diagram automorphism. Then

$$
\begin{aligned}
& \sigma_{w}\left(s c+\tau d+\sum_{j=1}^{r} u_{j} C^{j}{ }_{j}\right)=\left(s-r \tau / 4+\Sigma_{j=1}^{r} u_{j}\right) c+\tau d+\Sigma_{j=1}^{r}\left(\tau / 2-u_{r+1-j}\right) C_{j}^{j}, \\
& \sigma_{w}\left(s \delta+l \hat{\Lambda}_{0}+\sum_{j=1}^{r} y_{j} \varepsilon_{j}\right)=\left(s-l r / 4+\Sigma_{j=1}^{r} y_{j} / 2\right) \delta+l \hat{\Lambda}_{0}+\Sigma_{j=1}^{r}\left(l-y_{r+1-j}\right) \varepsilon_{j}
\end{aligned}
$$


In particular

$$
\begin{aligned}
& \sigma_{w}\left(Y+l \hat{\Lambda}_{0}\right) \equiv Y^{c}+l \hat{\Lambda}_{0} \bmod \mathbf{C} \delta \quad \text { for } \quad Y \in \mathscr{Y}_{r, l} . \\
& \underset{0}{\sqrt{0}} \cdots \longrightarrow \longrightarrow 0 \leftarrow 0
\end{aligned}
$$

\subsubsection{Type $D_{r}^{(1)}$}

(0)

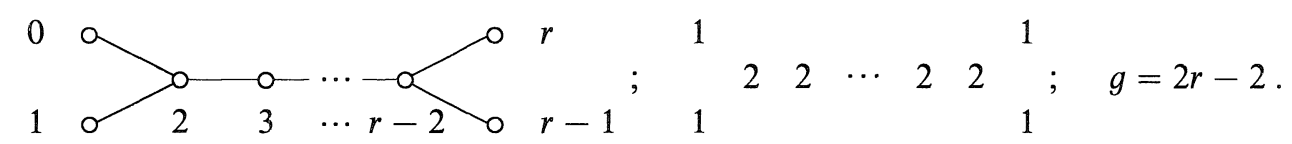

(1) $\hat{P}_{+}(L)=L \hat{\Lambda}_{0}+\left\{\begin{array}{l|l}\sum_{j=1}^{r} y_{j} \varepsilon_{j} & \begin{array}{l}\frac{L}{2} \geq \min \left(y_{1}, L-y_{1}\right) \geq y_{2} \geq \cdots \geq y_{r-1} \geq\left|y_{r}\right| \geq 0, \\ \left(y_{1}, \ldots, y_{r}\right) \in(\mathbb{Z}+s)^{r}, \quad s=0 \text { or } 1 / 2\end{array}\end{array}\right\}$

(2) $W \simeq \mathfrak{S}_{r} \ltimes\{ \pm 1\}^{r-1}, \quad M=\left\{\Sigma m_{j} \varepsilon_{j} \in \bigoplus_{j=1}^{r} \mathbb{Z} \varepsilon_{j} \mid \Sigma m_{j} \in 2 \mathbb{Z}\right\}$.

(3) For $u=\sum_{j=1}^{r} u_{j} D^{j} \in \mathfrak{h}, \lambda=\sum_{j=1}^{r} \lambda_{j} \varepsilon_{j} \in \mathfrak{h}^{*}$ and $s, s^{\prime}= \pm 1$,

$$
\begin{aligned}
& \Sigma_{a, b=0,1} s^{a} s^{\prime b} \mathscr{A}_{\sigma_{01}{ }^{a} \sigma_{r-1, r^{b}\left(\lambda+l \hat{\Lambda}_{0}\right)}}(\tau, u) \\
& =\operatorname{det}\left[\theta^{\mathbf{Z}, s s^{\prime}}{ }_{\lambda_{i}, l}\left(\tau, u_{j}\right)+s^{\prime} \theta^{\mathbf{Z}, s s^{\prime}}-\lambda_{i} \cdot l\right. \\
&
\end{aligned}
$$

(4) The diagram automorphisms $\sigma_{w}, \sigma_{0}=\sigma_{01}$ and $\sigma_{r}=\sigma_{r-1, r}$ that corresponds respectively to the permutations $w:=\left(\begin{array}{cccc}0 & \cdots & r-1 & r \\ r & \cdots & 1 & 0\end{array}\right),(0,1)$ and $(r-1, r)$ are given as follows.

$\sigma_{w}\left(s c+\tau d+\sum_{j=1}^{r} u_{j} D_{j}^{j}\right)=\left(s-r \tau / 8+\sum_{j=1}^{r} u_{j} / 2\right) c+\tau d+\Sigma_{j=1}^{r}\left(\tau / 2-u_{r+1-j}\right) D_{j}^{j}$, $\sigma_{w}\left(s \delta+L \hat{\Lambda}_{0}+\sum_{j=1}^{r} y_{j} \varepsilon_{j}\right)=\left(s-L r / 8+\sum_{j=1}^{r} y_{j} / 2\right) \delta+L \hat{\Lambda}_{0}+\sum_{j=1}^{r}\left(L / 2-y_{r+1-j}\right) \varepsilon_{j}$.

In particular $\sigma_{w}\left(Y+2 l \hat{\Lambda}_{0}\right) \equiv Y^{c}+2 l \hat{\Lambda}_{0} \bmod \mathbb{C} \delta$ for $Y \in \mathscr{Y}_{r, l}$.

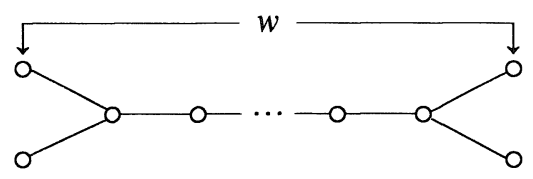

$$
\begin{aligned}
\sigma_{01}\left(s c+\tau d+\Sigma_{j=1}^{r} u_{j} D_{j}^{j}\right) & =\left(s-\tau / 2+u_{1}\right) c+\tau d+\left(\tau-u_{1}\right) D^{1}{ }_{1}+\Sigma_{j=2}^{r} u_{j} D_{j}^{j}, \\
\sigma_{01}\left(s \delta+L \hat{\Lambda}_{0}+\Sigma_{j=1}^{r} y_{j} \varepsilon_{j}\right) & =\left(s-L / 2+y_{1}\right) \delta+L \hat{\Lambda}_{0}+\left(L-y_{1}\right) \varepsilon_{1}+\Sigma_{j=2}^{r} y_{j} \varepsilon_{j} .
\end{aligned}
$$

$(0,1)$ :

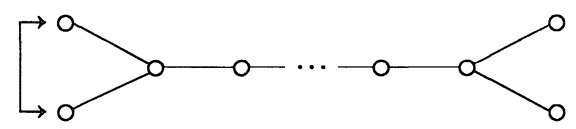




$$
\begin{gathered}
\sigma_{r-1, r}\left(s c+\tau d+\sum_{j=1}^{r} u_{j} D_{j}^{j}\right)=z c+\tau d+\left(\sum_{j=1}^{r-1} u_{j} D_{j}^{j}\right)-u_{r} D_{r}^{r}, \\
\sigma_{r-1, r}\left(s \delta+L \hat{\Lambda}_{0}+\sum_{j=1}^{r} y_{j} \varepsilon_{j}\right)=s \delta+L \hat{\Lambda}_{0}+\left(\sum_{j=2}^{r-1} y_{j} \varepsilon_{j}\right)-y_{r} \varepsilon_{r} .
\end{gathered}
$$

It holds that $\sigma_{01}=t_{\varepsilon_{1}} \circ r_{\varepsilon_{1}}$ and $\sigma_{r-1, r}=r_{\varepsilon_{r}}$ as an automorphism of $\tilde{\mathfrak{h}}^{*}$, where $t_{\varepsilon_{1}}$ and $r_{\varepsilon_{t}}$ are as in 1.5.3(4).

\subsubsection{Other affine diagrams and their dual labels [Kac]}

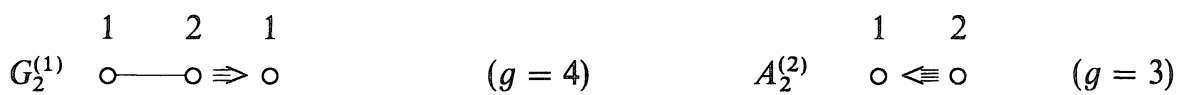

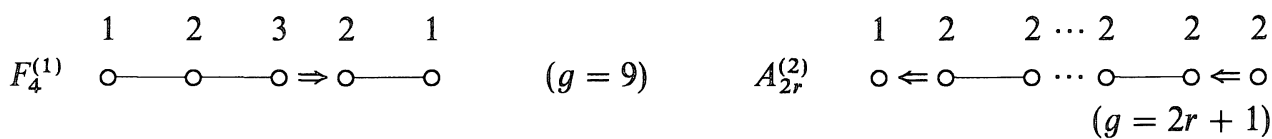
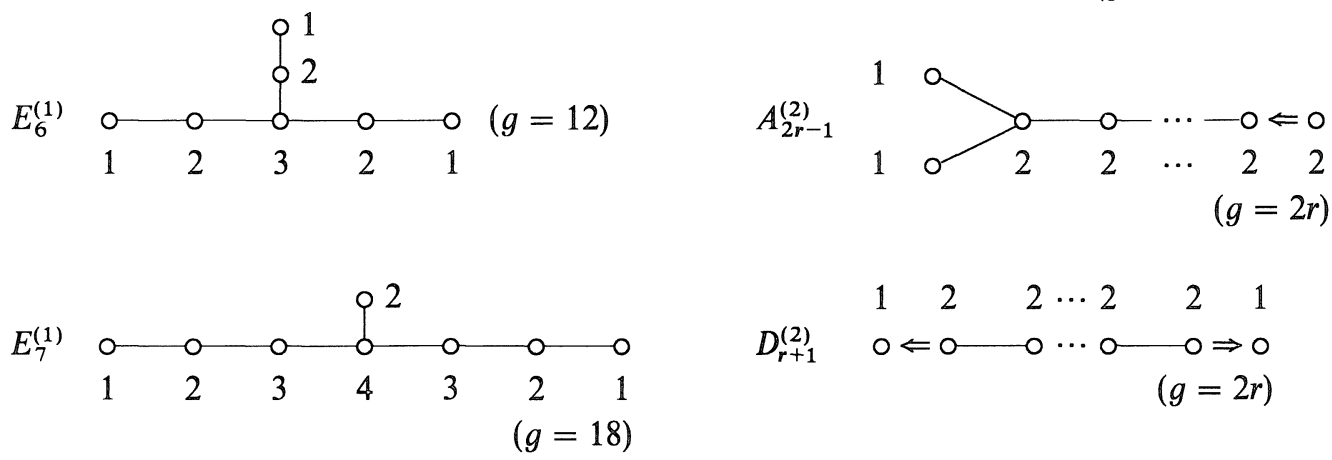

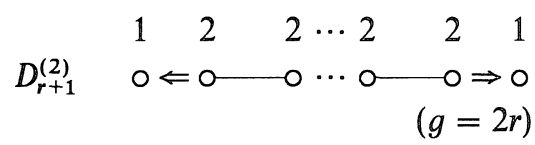

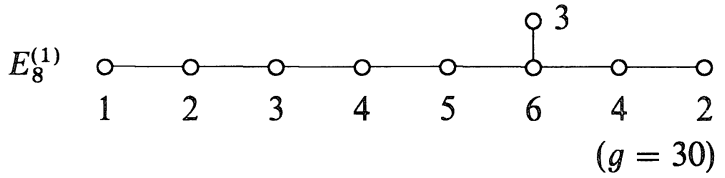

$$
\begin{array}{llllrr}
E_{6}^{(2)} & 1 & 2 & 3 & 4 & 2 \\
& 0 & 0 & 0 & 0 \\
& 1 & 2 & 3 & 0 \\
(g=12) & 0 \\
D_{4}^{(3)} & \circ & 0 & 0 &
\end{array}
$$

\section{§2. Clifford Algebras and Spin Representations}

2.0. We will briefly summarize about the spin representations ( $[\mathrm{B}],[\mathrm{F} 1],[\mathrm{FF}]$, $[\mathrm{KP} 1])$.

\subsection{Clifford algebra $C(W)$}

Let $W$ be a vector space with a non-degenerate symmetric bilinear form $\{$,$\} .$ Then the algebra 


$$
C(W):=T(W) /\langle v \otimes w+w \otimes v-\{v, w\}\rangle_{\text {two sided ideal }}
$$

is called the Clifford algebra associated to $(W,\{\}$,$) . Here T(W)$ denotes the tensor algebra over $W$. We write $u v$ for the residue class of $u \otimes v$ in $C(W)$. When $\operatorname{dim} W<\infty$, as a $\mathbf{C}$-algebra

$$
C(W) \simeq\left\{\begin{array}{ll}
\operatorname{Mat}\left(2^{n}, 2^{n}\right) & : \text { if } \operatorname{dim} W=2 n \\
\operatorname{Mat}\left(2^{n}, 2^{n}\right) \oplus \operatorname{Mat}\left(2^{n}, 2^{n}\right): & \text { if } \operatorname{dim} W=2 n+1
\end{array} .\right.
$$

\subsection{The spin module $\bigwedge\left(W^{-}\right)$of $C(W)$}

First, suppose there exists an isotropic decomposition $W=W^{+} \oplus W^{-}$, that is, $\left\{W^{ \pm}, W^{ \pm}\right\}=0$ and $\left.\{\}\right|_{,W^{+} \times W^{-}}$is nondegenerate (if $\operatorname{dim} W<\infty$, this means $W$ is even dimensional). Then the exterior algebra $\bigwedge\left(W^{-}\right)$, viewed as a $\bigwedge\left(W^{-}\right)$-module by the left multiplication, gives rise to an irreducible left $C(W)$-module by defining

$$
w^{+} \cdot 1:=0 \quad \text { for } \quad 1 \in \bigwedge\left(W^{-}\right) \text {and any } w^{+} \in W^{+} .
$$

This module is isomorphic to the quotient module $C(W) / C(W) W^{+}$by the correspondance $1 \leftrightarrow 1+C(W) W^{+}$, and its element is called a spinor. We denote the representation $C(W) \stackrel{\simeq}{\rightrightarrows}$ End $\bigwedge\left(W^{-}\right)$by $\diamond_{W}$.

Next suppose $W=W^{\prime} \oplus \mathbf{C} e$ be an orthogonal direct sum with $\{e, e\}=1$ and $W^{\prime}$ has an isotropic decomposition $W^{\prime}=W^{+} \oplus W^{-}$(in the following, we refer this type of decomposition to a quasi-isotropic decomposition). Then a $C\left(W^{\prime}\right)$-module $\bigwedge\left(W^{-}\right)$ becomes an irreducible $C(W)$-module by defining

$$
\sqrt{2} e \cdot v:= \pm(-1)^{p} \cdot v \quad \text { for } \quad v \in \bigwedge^{p}\left(W^{-}\right) .
$$

We denote this representation $C(W) \rightarrow$ End $\bigwedge\left(W^{-}\right)$by $s_{W}^{\frac{t}{W}}$.

In both cases, we call an element of $W^{-}$(resp. $W^{+}$) a creation operator (resp. annihilation operator).

\subsection{The spin module $\bigwedge\left(W_{N}^{-}\right)$of $\mathfrak{o}(N)$}

Suppose $W=W_{N}$ is of finite dimension $N$, and $\left\{e_{i}\right\}_{i=-n}^{n}$ is an orthonormal basis of $W_{N}:\left\{e_{i}, e_{j}\right\}=\delta_{i j}$.

(0) (Notice: Here and after we put $n:=[N / 2]$ and regard that the indeces $-n \leq$ $i, j, \cdots \leq n$ do not take zero if $N$ is even.)

Then the following map is a Lie algebra monomorphism.

$$
\beta: \mathfrak{o}\left(\mathbb{1}_{N}\right) \ni O_{j}^{i}:=E_{j}^{i}-E_{i}^{j} \mapsto e_{i} e_{j} \in C\left(W_{N}\right) .
$$

Taking a (quasi) isotropic decomposition of $W$ and considering $S_{N}:=\varsigma_{W_{N}}^{( \pm)} \circ \beta$, we get the spin representation of $\mathfrak{v}(N)$. (In case of odd $N, \varsigma_{W_{N}}^{+} \circ \beta \simeq \varsigma_{W_{N}}^{-} \circ \beta$ holds and we can write it $S_{N}$.) Put

$$
\left[\begin{array}{l}
\psi_{-j} \\
\psi^{-j}
\end{array}\right]:=\left[\begin{array}{l}
\psi^{j} \\
\psi_{j}
\end{array}\right]:=\frac{1}{\sqrt{2}}\left[\begin{array}{rr}
1 & \sqrt{-1} \\
1 & -\sqrt{-1}
\end{array}\right]\left[\begin{array}{c}
e_{j} \\
e_{-j}
\end{array}\right] \quad\left(\text { and } \psi^{0}:=\psi_{0}:=e_{0}\right)
$$


$(1 \leq j \leq n)$ to fix a (quasi) isotropic decomposition

$$
W_{N} \simeq W_{N}^{+} \oplus W_{N}^{-}\left(\oplus \mathbb{C} e_{0}\right), \quad \text { where } \quad W_{N}^{ \pm}:=\oplus_{j=1}^{[N / 2]} \mathbb{C} \psi^{ \pm j}
$$

Then for $i, j, k=-n, \ldots, n$ and $X=B$ or $D$, we have

$$
S_{N}\left(X_{j}^{i}\right)=: \psi^{i} \psi_{j}: \quad \text { and } \quad\left[S_{N}\left(X^{i}\right), \psi^{k}\right]=\delta_{j}^{k} \psi^{i}-\delta_{-k}^{i} \psi_{j} .
$$

for the root basis $X^{i}{ }_{j}(1.4 .3 \sim 4,(1.2))$, where : : is the normal product defined by

$$
: a b::=(a b-b a) / 2 \quad \text { for } \quad a, b \in W_{N} \text {. }
$$

Moreover the isomorphism $\bigwedge\left(W_{N}^{-}\right) \simeq \bigotimes_{j=1}^{n} \bigwedge\left(\mathbb{C} \psi_{j}\right)$ as a $\left(\bigoplus_{j} \mathbb{C} X^{j}{ }_{j}\right)$-module yields the following character formula.

Proposition 2.3. Putting $w_{j}:=e^{u_{j}}$ for $u_{j} \in \mathbb{C}$, we have

$$
\operatorname{Trace}_{\bigwedge\left(w_{\bar{N}}\right)} \exp \left[\Sigma_{j=1}^{n} u_{j} X_{j}^{j}\right]=\prod_{j=1}^{n}\left(w_{j}^{1 / 2}+w_{j}^{-1 / 2}\right) .
$$

There is a Hermitian form $H$ on $\bigwedge\left(W_{N}^{-}\right)$uniquely defined by

$$
H(1,1)=1 \quad \text { and } \quad H\left(e_{j} \cdot u, v\right)=H\left(u, e_{j} \cdot v\right)
$$

$\left(u, v \in \bigwedge\left(W_{N}^{-}\right), j= \pm 1 \cdots \pm n\right)$. We have $H\left(\psi^{j} \cdot u, v\right)=H\left(u, \psi_{j} \cdot v\right)$.

Theorem 2.3 [B]. (i) The Hermitian form $H$ is positive definite and contravariant with respect to the $\mathrm{o}(N)$-action.

(ii) If $N=2 n+1$, then as an $\mathfrak{v}(2 n+1)$-module

$$
\bigwedge\left(W_{2 n+1}^{-}\right) \simeq \mathbb{L}^{\mathrm{o}(2 n+1)}\left(\Lambda_{n}\right)
$$

The highest weight vector is given by $1 \in \bigwedge\left(W_{2 n+1}^{-}\right)$.

(iii) If $N=2 n$, then as an $\mathrm{o}(2 n)$-module

$$
\bigwedge^{\text {even }}\left(W_{2 n}^{-}\right) \simeq \mathbb{L}^{\mathrm{o}(2 n)}\left(\Lambda_{n}\right), \quad \Lambda^{\mathrm{odd}}\left(W_{2 n}^{-}\right) \simeq \mathbb{L}^{\mathrm{o}(2 n)}\left(\Lambda_{n-1}\right),
$$

where $\bigwedge^{\text {even }}(\cdot):=\bigoplus_{p \in 2 \mathbb{Z}} \bigwedge^{p}(\cdot)$ and $\bigwedge^{\text {odd }}(\cdot):=\bigoplus_{p \in 2 \mathbb{Z}+1} \bigwedge^{p}(\cdot)$. Hence $\bigwedge\left(W_{2 n}^{-}\right)$gives $a\left\langle\sigma_{n-1, n}\right\rangle \ltimes \mathfrak{o}(2 n)$-module by 1.3.2(9) and we have

$$
\bigwedge\left(W_{2 n}^{-}\right) \simeq \mathbb{L}^{\left\langle\sigma_{n-1, n}\right\rangle \times 0(2 n)}\left(\Lambda_{n}\right) .
$$

The highest weight vector of $\bigwedge^{\mathrm{even}}\left(W_{2 n}^{-}\right)$(resp. $\left.\bigwedge^{\mathrm{odd}}\left(W_{2 n}^{-}\right)\right)$with respect to $\mathrm{o}(n)$ is given by $1\left(\right.$ resp. $\left.\psi_{n}\right) \in \bigwedge\left(W_{2 n}^{-}\right)$.

\subsection{The spin modules $\bigwedge\left(W_{N}^{\mathrm{Z}+h,-}\right)$ of $\mathfrak{o}(N)^{\wedge}$}

We retain the notation in the previous paragraph. For $h=0$ or $1 / 2$, we define a vector space with the inner product

$$
W_{N}^{\mathbb{Z}+h}:=W_{N} \otimes t^{h} \mathbb{C}\left[t, t^{-1}\right] \quad \text { with } \quad\{a(\mu), b(v)\}:=\{a, b\} \delta_{\mu+v, 0},
$$

where $a, b \in W_{N}, \mu, v \in \mathbb{Z}+h$ and $a(\mu):=a \otimes t^{\mu}$. Given a (quasi) isotropic decomposition $W_{N}=W_{N}^{+} \oplus W_{N}^{-}\left(\oplus \mathbb{C} e_{0}\right)$ of $W_{N}(2.3(3))$, we choose an (quasi) isotropic 
decomposition

$$
W_{N}^{\mathbf{Z}+h}=W_{N}^{\mathbf{Z}+h,+} \oplus W_{N}^{\mathbf{Z}+h,-}\left(\oplus \mathbf{C} e^{0}\right),
$$

where

$$
W_{N}^{\mathbf{Z}+h, \pm}:= \begin{cases}W_{N} \otimes t^{ \pm 1 / 2} \mathbf{C}\left[t^{ \pm 1}\right] & \text { if } h=\frac{1}{2} \\ \left(W_{N} \otimes t^{ \pm 1} \mathbf{C}\left[t^{ \pm 1}\right]\right) \oplus\left(W_{N}^{ \pm} \otimes t^{0}\right) & \text { if } h=0\end{cases}
$$

and

$$
e^{0}:= \begin{cases}e_{0}(0) & \text { if } h=0 \text { and } N:=\text { odd } \\ 0 & \text { otherwise }\end{cases}
$$

We define the normal product for $a(\mu), b(v) \in{W_{N}}^{\mathbf{Z}+h}$ by

$$
: a(\mu) b(v)::= \begin{cases}-b(v) a(\mu) & \text { if } \mu>0>v \\ \{a(\mu) b(v)-b(v) a(\mu)\} / 2 & \text { if } \mu=0=v \\ a(\mu) b(v) & \text { otherwise } .\end{cases}
$$

There is a Hermitian form $H$ on $\bigwedge\left(W_{N}^{\mathrm{Z}+h,-}\right)$ determined by

$$
H(1,1)=1 \quad \text { and } \quad H\left(e_{j}(v) \cdot u, v\right)=H\left(u, e_{j}(-v) \cdot v\right) .
$$

Theorem 2.4a.(i) [F1]. The following map is a Lie monomorphism:

$$
\begin{aligned}
S_{N}^{\mathbf{Z}+h}=S_{N}^{\wedge}: \mathfrak{o}\left(\mathbb{1}_{N}\right)^{\wedge} \ni O^{i}(m) \mapsto \Sigma_{\mu+v=m}: e_{i}(\mu) e_{j}(v): \in \text { End } \bigwedge\left(W_{N}{ }^{\mathbf{Z}+h,-}\right), \\
c \mapsto i d .
\end{aligned}
$$

(ii) For $x(m) \in \mathfrak{o}(N)^{\wedge}$ and $\psi \in W_{N}$,

$$
\left[S_{N}^{\wedge}(x(m)), \psi(k)\right]=\left[S_{N}(x), \psi\right](m+k) \quad \text { on } \bigwedge\left(W_{N}^{\mathrm{Z}+h,^{-}}\right) .
$$

(iii) Let $D_{n}:=D^{\mathrm{o}(N)^{\wedge}}(n)(n \in \mathbb{Z})$ be the Virasoro operaters associated to the representation $S_{N}^{\mathrm{Z}+h}(1.2 .2)$. Then

$$
\left[D_{n}, \psi(k)\right]=-\left(\frac{n}{2}+k\right) \psi(k+n)
$$

for $\psi \in W_{N}$.

(iv) The Hermitian form $H(6)$ is positive definite and contravariant with respect to the $\mathfrak{v}\left(\mathbf{1}_{N}\right)^{\wedge}$-action in (i).

The sum $\Sigma: e_{i}(\mu) e_{j}(v)$ : above should be understood as $\Sigma \jmath^{( \pm)}\left(: e_{i}(\mu) e_{j}(v):\right)$, where $\jmath^{( \pm)}$ denotes the spin representation of $C\left(W_{N}^{\mathbf{Z}+h}\right)$. As is similar to $\S 2.3, \pm$ give equivalent representations in case of $W_{2 n+1}^{\mathbf{Z}} \ni e^{0}=e_{0}(0) \neq 0$.

The representation $S_{N} \hat{N}=S_{N}^{Z+h}$ is called the spin representation.

Put

$$
\operatorname{ch}_{N} \pm, \mathbf{Z}+h:=\operatorname{ch}_{\bigwedge_{\text {even }}\left(W_{N} \mathrm{Z}^{+h,-}\right)} \pm \operatorname{ch}_{\bigwedge_{\text {odd }}\left(W_{N} \mathrm{Z}^{+h,-}\right)}
$$

(see 1.2.3(6) for $\mathrm{ch}$.). As in $\S 2.3$ we have the followings. 
Proposition 2.4a [F1]. The characters $\mathrm{ch}_{\bar{N}}^{ \pm, \mathrm{Z}+h}$ have the following formulae:

$$
\begin{aligned}
& \operatorname{ch}_{2 n}^{ \pm, \mathbb{Z}}\left(\tau D_{0}+\sum_{j=1}^{n} u_{j} D_{j}^{j}\right)=\prod_{j=1}^{n} \frac{-i \theta\left(\tau, u_{j}+\frac{1 \pm 1}{4}\right)}{\eta(\tau)} \\
& \operatorname{ch}_{2 n}^{ \pm, Z+1 / 2}\left(\tau D_{0}+\sum_{j=1}^{n} u_{j} D_{j}^{j}\right)=\prod_{j=1}^{n} \frac{\hat{\theta}\left(\tau, u_{j}+\frac{1 \pm 1}{4}\right)}{\eta(\tau)} \\
& \operatorname{ch}_{2 n+1}^{ \pm, \mathbb{Z}}\left(\tau D_{0}+\sum_{j=1}^{n} u_{j} B^{j}\right)=H^{ \pm, 0}(\tau) \prod_{j=1}^{n} \frac{-i \theta\left(\tau, u_{j}+\frac{1 \pm 1}{4}\right)}{\eta(\tau)} \\
& \operatorname{ch}_{2 n+1}^{ \pm, \mathbb{Z}+1 / 2}\left(\tau D_{0}+\sum_{j=1}^{n} u_{j} B_{j}^{j}\right)=H^{ \pm, 1 / 2}(\tau) \prod_{j=1}^{n} \frac{\hat{\theta}\left(\tau, u_{j}+\frac{1 \pm 1}{4}\right)}{\eta(\tau)}
\end{aligned}
$$

where $\theta$ (resp. $\hat{\theta}, \eta)$ is given by 1.5.1(12) (resp. ibid. (13), (14)) and

$$
\begin{aligned}
H^{+, 0}(\tau):=\frac{\eta(2 \tau)}{\eta(\tau)}, & H^{+, 1 / 2}(\tau):=\frac{\eta(\tau)^{2}}{\eta(2 \tau) \eta(\tau / 2)}, \\
H^{-, 0}(\tau):=\eta(\tau), & H^{-, 1 / 2}(\tau):=\frac{\eta(\tau / 2)}{\eta(\tau)}
\end{aligned}
$$

(i.e. $\quad H^{ \pm, 0}(\tau)=q^{1 / 24} \prod_{n \geq 1}\left(1 \pm q^{n}\right), \quad H^{ \pm, 1 / 2}(\tau)=q^{-1 / 48} \prod_{n \geq 1}\left(1 \pm q^{n-1 / 2}\right) \quad$ with $\quad q:=$ $\exp (2 \pi i \tau))$.

Theorem 2.4b [F1]. Suppose $N \geq 2$.

(i) If $h=1 / 2$, then

$$
\begin{aligned}
& \bigwedge^{\text {even }}\left(W_{N}^{\mathrm{Z}+1 / 2,-}\right) \simeq \mathbb{L}^{\mathrm{o}(N) \wedge}(0 ; 1)=\mathbb{L}^{\mathrm{o}(N) \wedge}\left(\hat{\Lambda}_{0}\right), \\
& \bigwedge^{\text {odd }}\left(W_{N}^{\mathrm{Z}+1 / 2,-}\right) \simeq \mathbb{L}^{\mathrm{o}(N) \wedge}\left(\Lambda_{1} ; 1\right)=\mathbb{L}^{\mathrm{o}(N)^{\wedge}}\left(\hat{\Lambda}_{1}\right) .
\end{aligned}
$$

The highest weight vector is respectively given by $1, \psi^{1}\left(-\frac{1}{2}\right) \cdot 1$.

(ii) If $h=0$ and $N=2 n$, then

$$
\begin{aligned}
& \bigwedge^{\text {even }}\left(W_{2 n}{ }^{\mathbb{Z},-}\right) \simeq \mathbb{L}^{\mathrm{p}(2 n)^{\wedge}}\left(\Lambda_{n} ; 1\right)=\mathbb{L}^{\mathrm{o}(2 n)^{\wedge}}\left(\hat{\Lambda}_{n}\right), \\
& \bigwedge^{\text {odd }}\left(W_{2 n}{ }^{\mathrm{Z},-}\right) \simeq \mathbb{L}^{\mathrm{o}(2 n)^{\wedge}}\left(\Lambda_{n-1} ; 1\right)=\mathbb{L}^{\mathrm{o}(2 n)^{\wedge}}\left(\hat{\Lambda}_{n-1}\right) \text {. }
\end{aligned}
$$

The highest weight vector is respectively given by $1, \psi_{n}(0) \cdot 1$.

(iii) If $h=0$ and $N=2 n+1$, then

$$
\bigwedge\left(W_{2 n+1} \mathbf{Z},-\right) \simeq \mathbb{L}^{\mathfrak{D}(2 n+1)^{\wedge}}\left(\Lambda_{n} ; 1\right)=\mathbb{L}^{\mathfrak{D}(2 n+1)^{\wedge}}\left(\hat{\Lambda}_{n}\right) .
$$

The highest weight vector is given by 1 .

Following the notation of 1.3.2(2), we therefore have

$$
\bigwedge\left(W_{N}^{\mathrm{Z}+h,-}\right) \simeq \mathbb{L}^{\left\langle\sigma_{01}\right\rangle \times \mathrm{o}(N)^{\wedge}}(0 ; 1), \quad \text { etc. }
$$


On $\bigwedge\left(W_{N}^{\mathrm{Z}+h,-}\right)$ there are two actions of Virasoro algebra: one is the SegalSugawara operators $D_{m}=D^{\mathrm{o}(N)^{\wedge}}(m)(1.2 .2(3))$ obtained by $S_{N}^{\wedge}$, and the other is what defined by

$$
\mathscr{V}_{i} \ni d(m) \mapsto D^{C l i f}(m), \quad z \mapsto \frac{N}{2} i d \in \text { End } \bigwedge\left(W_{N}{ }^{\mathbb{Z}+h,-}\right)
$$

where

$$
D^{C l i f}(m):=-\frac{6 h-2}{48} \delta_{m, 0} N i d-\frac{1}{4} \sum_{j=1}^{N} \sum_{\mu+v=m}(\mu-v): e_{j}(\mu) e_{j}(v):,
$$

which satisfies the followings ([FF, (5.17)]).

$$
\begin{aligned}
& {\left[D^{\text {Clif }}(m), a(v)\right]=-\left(\frac{m}{2}+v\right) a(m+v) \quad\left(a \in W_{N}\right)} \\
& {\left[D^{C l i f}(m), X(n)\right]=-n X(m+n) \quad(X \in \mathfrak{p}(N))}
\end{aligned}
$$

Proposition 2.4b [FF, Proposition 13]. For $N \geq 2$, we assert

$$
D^{\mathfrak{o}(N)^{\wedge}}(m)=D^{\text {Clif }}(m) \quad \text { for all } m \in \mathbb{Z} .
$$

Proof. In view of Proposition 1.2 and the above commutation relation (17), the assertion follows from the central charge identity

$$
\frac{\operatorname{dim} \mathfrak{o}(N)}{1+g}=\frac{N(N-1) / 2}{1+(N-2)}=\frac{N}{2} .
$$

Remark 2.4. (i) In the case of $N=1$, the spin module $\bigwedge\left(W_{1}^{\mathrm{Z}+h_{-}-}\right)$, viewed as a Virasoro module by (15), decomposes as follows.

$$
\begin{aligned}
\bigwedge^{\text {even }}\left(W_{1}^{\mathrm{Z}+1 / 2,-}\right) & \simeq \mathbb{L}^{\mathscr{V}_{i z}}(0,1 / 2) \\
\bigwedge^{\text {odd }}\left(W_{1}^{\mathrm{Z}+1 / 2,-}\right) & \simeq \mathbb{L}^{\mathscr{V}_{i r}}(1 / 2,1 / 2) \\
\bigwedge\left(W_{1}^{\mathrm{Z},-}\right) & \simeq \mathbb{L}^{\mathscr{V}^{i}}(1 / 16,1 / 2)
\end{aligned}
$$

See Example 5.3 for the definition of $\mathbb{L}^{\mathscr{V} i n}(h, c)$, where we also review their character formula (5.3.(6)). These facts are shown by the character identities

$$
H^{+, 0}(\tau)=\operatorname{ch}_{\mathbb{L}^{r(1 / 1 / 16,1 / 2)}}(\tau)
$$

and

$$
H^{ \pm, 1 / 2}(\tau)=\operatorname{ch}_{\mathbf{L}^{\gamma_{\imath \tau}(0,1 / 2)}}(\tau) \pm \operatorname{ch}_{\mathbf{L}^{\gamma_{\imath t}(1 / 2,1 / 2)}}(\tau),
$$

all of which follows from the celebrated Euler identity (1.5.1(14))

$$
\prod_{n=1}^{\infty}\left(1-q^{n}\right)=\Sigma_{n \in \mathbb{Z}}(-1)^{n} q^{\left(3 n^{2}+n\right) / 2}
$$

or

$$
\prod_{n=1}^{\infty}\left(1-(-q)^{n}\right)=\Sigma_{n \in \mathbf{Z}}(-1)^{n(n+1) / 2} q^{\left(3 n^{2}+n\right) / 2} .
$$


(ii) In the case of $N=2$, putting $\check{h}:=1 / 2-h$ we have the following isomorphism, which is equivalent to the Jacobi triple product identity $(1.5 .1(12 \sim 13))$ in terms of characters.

$$
\bigwedge\left(W_{2}{ }^{\mathbf{Z}+h,-}\right) \simeq \mathbb{L}^{\mathbf{Z} \varepsilon \times \mathfrak{o}(2)^{\wedge}}(\check{h} \varepsilon, 1)
$$

Here $\mathbb{Z} \ltimes \mathfrak{o}(2)^{\wedge}=\mathbb{Z} \varepsilon \ltimes\left(C D^{1}{ }_{1}\right)_{B}^{\wedge}$ denotes the Heisenberg system (1.3.3(1)) defined by

$$
D^{1}{ }_{1}:=\left[\begin{array}{rr}
1 & 0 \\
0 & -1
\end{array}\right], \quad B\left(D^{1}{ }_{1}, D^{1}{ }_{1}\right):=1 \quad \text { and } \quad \varepsilon\left(D^{1}{ }_{1}\right)=1,
$$

and $\mathbb{L}^{\mathbf{Z}_{\varepsilon \ltimes \mathfrak{o}(2)^{\wedge}}}(\lambda, l)$ denotes its Boson Fock space representation $(1.3 .3(2) \sim(4))$. See also $\S 8$.

(iii) To calculate the relations such as (7), (8), (9), (16), (17) and (18), the method of operator product expansion and the so-called Wick's theorem are quite useful. See e.g. $[\mathrm{FF}]$ for them.

\section{§3. Dual Pairs of Classical Lie Algebras}

3.0. In the following paragraphs $3.1 \sim 3.2$ we state our main result in finite dimensional cases. We give their proof in 3.3.

\subsection{Definition of representations}

Here we define the representations of some pairs of Lie algebras, which turn out to form dual pairs $(\S 0.1)$.

\subsubsection{Case AA. $\left(\mathfrak{g}_{\mathrm{L}}, \mathfrak{g}_{\mathrm{R}}\right):=(\mathfrak{g l}(l)$, $\mathfrak{s l}(r))$ on $\bigwedge\left(W_{2 l r}^{-}\right)$}

Let $V_{l}$ and $V_{r}$ be vector spaces of dimension $l$ and $r$ respectively and put $V_{l r}:=V_{l} \otimes V_{r}$. We have the following three Lie algebra monomorphisms.

$$
\begin{aligned}
& \mathrm{L}^{\prime}: \operatorname{gl}\left(V_{l}\right) \ni x \mapsto x \otimes \mathbb{1}_{r} \in \mathfrak{g l}\left(V_{l r}\right) \\
& \mathrm{R}^{\prime}: \operatorname{gl}\left(V_{r}\right) \ni x \mapsto \mathbb{1}_{l} \otimes x \in \mathfrak{g l}\left(V_{l r}\right) \\
& i: \operatorname{gl}\left(V_{l r}\right) \ni x \mapsto \operatorname{diag}\left(x,-{ }^{t} x\right) \in \mathfrak{o}\left(W_{2 l r},\{, \quad\}\right)
\end{aligned}
$$

Here $W_{2 l r}:=V_{l r} \oplus V_{l r}^{*}$ and $\{$,$\} denotes the inner product induced by the pairing$ between $V_{l r}$ and its dual $V_{l r}^{*}$. Clearly it holds

$$
\left[\mathrm{L}^{\prime}(\operatorname{gl}(l)), \mathrm{R}^{\prime}(\mathfrak{g l}(r))\right]=0 \quad \text { and } \quad \mathrm{L}^{\prime}(\operatorname{gl}(l)) \cap \mathrm{R}^{\prime}(\mathfrak{s l}(r))=0 .
$$

Hence we get mutually commuting representations of $\mathfrak{g l}(l)$, sl $(r)$ by composing the maps $\mathrm{L}:=i \circ \mathrm{L}^{\prime}, \mathrm{R}:=i \circ \mathrm{R}^{\prime}$ and the spin representation $S$ of $\mathfrak{o}\left(W_{2 l r}\right) \simeq \mathfrak{o}(2 l r)(2.4(7))$ respectively:

$$
S \circ \mathrm{L}: \mathfrak{g l}(l) \rightarrow \text { End } \bigwedge\left(W_{2 l r}^{-}\right), \quad S \circ \mathrm{R}: \mathfrak{s l}(r) \rightarrow \text { End } \bigwedge\left(W_{2 l r}^{-}\right)
$$


We shall describe these representations explicitly. Take a basis $\left\{v_{j}\right\}_{j=1}^{l}$ of $V_{l}^{*}$ (resp. $\left\{v_{p}\right\}_{p=1}^{r}$ of $V_{r}^{*}$ ) which is dual to the basis $\left\{v^{j}\right\}_{j=1}^{l}$ of $V_{l}$ (resp. $\left\{v^{p}\right\}_{p=1}^{r}$ of $V_{r}$ ) (§1.1), and put $\psi^{j, p}:=v^{j} \otimes v^{p}, \psi_{j, p}:=v_{j} \otimes v_{p}(1 \leq j \leq l, 1 \leq p \leq r)$. Then $\left\{\psi^{j, p}\right\} \cup\left\{\psi_{j, p}\right\}$ gives a basis of $W_{2 l r}$ that satisfy

$$
\left\{\psi^{j, p}, \psi_{k, q}\right\}=\delta^{j}{ }_{k} \delta^{p}{ }_{q} \quad \text { and } \quad\left\{\psi^{j, p}, \psi^{k, q}\right\}=0=\left\{\psi_{j, p}, \psi_{k, q}\right\} .
$$

We fix an isotropic decomposition $W_{2 l r}=W_{2 l r}^{+} \oplus W_{2 l r}^{-}$given by

$$
W_{2 l r}^{+}=V_{l r}=\bigoplus \mathbf{C} \psi^{j, p} \quad \text { and } \quad W_{2 l r}^{-}=V_{l r}^{*}=\bigoplus \mathbf{C} \psi_{j, p}
$$

and put $\psi^{-j,-p}:=\psi_{j, p} . \quad$ Recall that $: a b:=(a b-b a) / 2(2.3(5))$.

Proposition 3.1.AA. We have the following formulae.

and

(i)(L) $S \circ \mathrm{L}\left(E^{j}{ }_{k}\right)=\Sigma_{p=1}^{r}: \psi^{j, p} \psi_{k, p}: \in C\left(W_{2 l r}\right) \simeq$ End $\bigwedge\left(W_{2 l r}^{-}\right)$for $E^{j}{ }_{k} \in \operatorname{gl}(l)=\operatorname{gl}\left(V_{l}\right)$,

(R) $S \circ \mathrm{R}\left(E_{q}^{p}\right)=\Sigma_{j=1}^{l}: \psi^{j, p} \psi_{j, q}: \in C\left(W_{2 l r}\right) \simeq$ End $\bigwedge\left(W_{2 l r}^{-}\right)$for $E_{q}^{p} \in \mathfrak{s l}(r)$.

(ii) $\left[S \circ \mathrm{L}\left(E_{j}^{i}\right), \psi^{k, p}\right]=\delta_{j}^{k} \psi^{i, p}-\delta_{-k}^{i} \psi_{j,-p},\left[S \circ \mathrm{R}\left(E^{p}{ }_{q}\right), \psi^{k, s}\right]=\delta_{q}^{s} \psi^{k, p}-\delta^{p}{ }_{-s} \psi_{k,-q}$.

Remark 3.1.1. Consider the case $r=1$. Let $v^{1}, \ldots, v^{l}$ be a basis of $\mathbf{C}^{l}$ in $\S 1.1$ that satisfies $E_{j}^{i} v^{k}=v^{i} \delta_{j}^{k}$ for $E_{j}^{i} \in \operatorname{gl}(l)$. The isomorphism

$$
\bigwedge\left(W_{2 l}^{-}\right) \ni \psi_{i_{1}} \ldots \psi_{i_{k}} \leftrightarrow v^{i_{1}} \ldots v^{i_{k}} \in \bigwedge\left(\mathbf{C}^{l}\right)
$$

between vector spaces induces that of their endomorphisms

$$
I: \text { End } \bigwedge\left(W_{2 l}^{-}\right) \stackrel{\cong}{\rightrightarrows} \text { End } \bigwedge\left(\mathbf{C}^{l}\right),
$$

and we have the following commutative diagram.

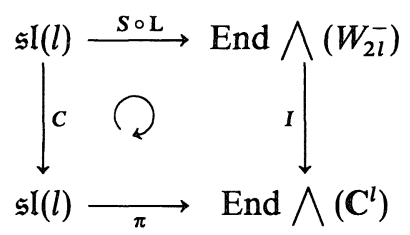

Here we denote by $C$ (resp. $\pi$ ) the Chevalley involution $X \mapsto-{ }^{t} X$ (resp. the exterior product representation). In fact, using the formula 3.1.AA(ii) we have the following, which shows that $S \circ \mathrm{L}$ coinsides with $\pi \circ C$ as representations of $\mathfrak{s l}(l)$.

$$
\begin{aligned}
\left(S \circ \mathrm{L}\left(E_{k}^{j}\right)\left(\psi_{i_{1}} \ldots \psi_{i_{p}}\right)\right. & =-: \psi_{k} \psi^{j}: \psi_{i_{1}} \ldots \psi_{i_{p}} \\
& =\left(-\psi_{k} \psi^{j}+\delta_{k}^{j} / 2\right) \psi_{i_{1}} \ldots \psi_{i_{p}} \\
& =\left(\delta_{k}^{j} / 2\right) \psi_{i_{1}} \ldots \psi_{i_{p}}+\sum_{s=1}^{k} \psi_{i_{1}} \ldots \psi_{i_{s-1}}\left[-\psi_{k} \psi^{j}, \psi_{i_{s}}\right] \psi_{i_{s+1}} \ldots \psi_{i_{p}} \\
& =\left(\delta_{k}{ }^{j} / 2\right) \psi_{i_{1}} \ldots \psi_{i_{p}}+\Sigma_{s=1}^{k} \psi_{i_{1}} \ldots \psi_{i_{s-1}}\left(-\psi_{k} \delta_{i_{s}}^{j}\right) \psi_{i_{s+1}} \ldots \psi_{i_{p}} .
\end{aligned}
$$

It also holds that $\bigwedge\left(W_{2 l r}^{-}\right) \simeq \bigwedge\left(W_{2 l}^{-}\right)^{\otimes r}$ (resp. $\bigwedge\left(W_{2 l r}^{-}\right) \simeq \bigwedge\left(W_{2 r}^{-}\right)^{\otimes l}$ ) as a $\operatorname{gl}(l)$ (resp. $\mathrm{gl}(r)-)$ module. This isomorphism is given by the assignation

$$
\bigwedge\left(W_{2 l}^{-}\right)^{\otimes r} \ni a^{(1)} \otimes \cdots \otimes a^{(r)} \stackrel{\simeq}{\cong} a^{1} \wedge \cdots \wedge a^{r} \in \bigwedge\left(W_{2 l r}^{-}\right),
$$


where for $a^{(p)}=\psi^{i} \wedge \cdots \wedge \psi^{j} \in \wedge\left(W_{2 l}^{-}\right)^{(p)}$ (the $p$-th copy) we put

$$
a^{p}:=\psi^{i, p} \wedge \cdots \wedge \psi^{j, p} \in \wedge\left(W_{2 l r}^{-}\right) .
$$

\subsubsection{Case $\mathbb{C} C$. $\left(\mathfrak{g}_{\mathrm{L}}, \mathfrak{g}_{\mathrm{R}}\right):=(\mathfrak{s p}(2 l), \mathfrak{s p}(2 r))$ orl $\wedge\left(W_{4 l r}^{-}\right)$}

Let $\mathbb{V}_{2 l}$ and $V_{2 r}$ respectively be a $2 l$ and $2 r$ dimensional vector space with an alternating bilinear form (, ) of maximal rank. Then $W_{4 l r}:=V_{2 l} \otimes V_{2 r}$ has a nondegenerate symmetric bilinear form $\{u \otimes x, v \otimes y\}:=(u, v)_{2 l} \cdot(x, y)_{2 r}$ and we have the following Lie algebra monomorphisms.

$$
\begin{aligned}
& \mathbb{L}: \mathfrak{s p}(2 l) \ni x \mapsto x \otimes \mathbb{R}_{2 r} \in \mathfrak{v}\left(W_{4 l r},\{, \quad\}\right) \\
& \mathbb{R}: \mathfrak{s p}(2 r) \ni x \mapsto \mathbb{Z}_{2 l} \otimes x \in \mathfrak{v}\left(W_{4 l r},\{,\}\right)
\end{aligned}
$$

We have $[\mathbb{L}(\mathfrak{s p}(2 l)), \mathbb{R}(\mathfrak{s p}(2 r))]=0$ and $\mathbb{L}(\mathfrak{s p}(2 l)) \cap \mathbb{R}(\mathfrak{s p}(2 r))=0$. Therefore we obtain mutually commuting representations

$$
S \circ \operatorname{L}: \mathfrak{s p}(2 l) \rightarrow \text { End } \bigwedge\left(W_{4 l r}^{-}\right) \quad \text { and } \quad S \circ \mathbb{R}: \mathfrak{s p}(2 r) \rightarrow \operatorname{End} \bigwedge\left(W_{4 l r}^{-}\right)
$$

of $\mathfrak{s p}(2 l)$ and $\mathfrak{s p}(2 r)$ respectively, where $S$ is the spin representation of $\mathfrak{o}\left(\mathbb{W}_{4 l r},\{\},\right) \simeq$ o $(4 l r)$ on $\bigwedge\left(W_{4 l r}^{-}\right)(2.4(7))$.

Explicitly these representations are given as follows. Choose a basis $\left\{v^{j}=v_{-j}\right\}_{j=-m}^{m}$ of $\left(V_{2 m},(,)_{2 m}\right) \simeq \mathbb{C}^{2 m}$ such that $\left(v^{j}, v_{k}\right)_{2 m}=-\left(v_{k}, v^{j}\right)_{2 m}=\delta^{j}{ }_{k}(m=l, r)$. Then we have a basis $\left\{\psi^{j, p}:=\psi_{-j,-p}:=v^{j} \otimes v^{p} \mid j= \pm 1, \ldots, \pm l, p= \pm 1, \ldots, \pm r\right\}$ of $W_{4 l r}$ that satisfy

$$
\left\{\psi^{j, p}, \psi_{k, q}\right\}=\delta_{j k} \delta_{p q}, \quad \text { where } \quad \psi_{j, p}:=\psi^{-j,-p},
$$

and an isotropic decomposition

$$
W_{4 l r}=W_{4 l r}^{+} \oplus W_{4 l r}^{-}, \quad \text { where } \quad W_{4 l r}^{ \pm}:=\bigoplus_{j>0, p} \mathbb{C} \psi^{ \pm j, p} .
$$

Proposition 3.1.CC。 For ${C^{j}}^{j} \in \mathfrak{s p}(2 l)(1.4 .3(1.2))(j, k= \pm 1, \ldots, \pm l)$,

$$
S \circ \mathbb{L}\left(C^{j}{ }_{k}\right)=\sum_{p=1}^{r}: \psi^{j, p} \psi_{k, p}-\operatorname{sgn}(j k) \psi^{-k, p} \psi_{-j, p}: \in \mathbb{E n d} \bigwedge\left(W_{4 l r}^{-}\right)
$$

and for $C^{p}{ }_{q} \in \mathfrak{s p}(2 r)(p, q= \pm 1, \ldots, \pm r)$ we have

$$
S \circ \mathbb{R}\left(C_{q}^{p}\right)=\sum_{j=1}^{l}: \psi^{j, p} \psi_{j, q}-\operatorname{sgn}(p q) \psi^{j,-q} \psi_{j,-p}: \in \text { End } \bigwedge\left(W_{4 l r}^{-}\right) .
$$

As in Case $\mathrm{AA}, \bigwedge\left(W_{4 l r}^{-}\right) \simeq \bigwedge\left(\mathbb{C}^{2 r}\right)^{\otimes l}$ as an $\mathfrak{s p}(2 r)$-module.

3.1.3. Case OO. $\left(\mathfrak{g}_{\mathrm{L}}, \mathfrak{g}_{\mathrm{R}}\right):=(\mathfrak{o}(L), \mathfrak{o}(R))$ on $\bigwedge\left(W_{L R}^{-}\right)$

For $M=L, R \in \mathbb{Z}_{>0}$, let $W_{M}$ be the $M$-dimensional vector space with nondegenerate symmetric bilinear form $\{$,$\} . There are following Lie algebra monomorphisms (See$ $\S 1.4 .6$ for $\left.\mathfrak{o}\left(\mathbb{1}_{M}\right)\right)$.

$$
\begin{aligned}
& \mathrm{L}: \mathfrak{o}\left(W_{L}\right) \simeq \mathfrak{v}\left(\mathbb{1}_{L}\right) \ni x \mapsto x \otimes \mathbb{1}_{R} \in \mathfrak{v}\left(W_{L} \otimes W_{R}\right) \simeq \mathfrak{v}\left(\mathbb{1}_{L R}\right) \\
& \mathrm{R}: \mathfrak{v}\left(W_{R}\right) \simeq \mathfrak{v}\left(\mathbb{R}_{R}\right) \ni x \mapsto \mathbb{1}_{L} \otimes x \in \mathfrak{v}\left(W_{L} \otimes W_{R}\right) \simeq \mathfrak{v}\left(\mathbb{R}_{L R}\right) .
\end{aligned}
$$


We have $\left[\mathrm{L}\left(\mathfrak{o}\left(\mathbb{1}_{L}\right)\right), \mathbb{R}\left(\mathfrak{o}\left(\mathbb{1}_{R}\right)\right)\right]=0, \quad L\left(\mathfrak{o}\left(W_{L}\right)\right) \cap \mathbb{R}\left(\mathfrak{o}\left(W_{R}\right)\right)=0$. Putting $W_{L R}:=W_{L} \otimes W_{R}$ we obtain mutually commuting representations

$$
S \circ \mathrm{L}: \mathfrak{o}(L) \rightarrow \text { End } \bigwedge\left(W_{L R}^{-}\right) \quad \text { and } \quad S \circ \mathrm{R}: \mathfrak{o}(R) \rightarrow \text { End } \bigwedge\left(W_{L R}^{-}\right),
$$

where $S$ is the spin representation of $\mathfrak{o}\left(W_{L R}\right)$.

Put $m:=\left[\frac{M}{2}\right]$ and let $\left\{e_{j}\right\}_{j=-m}^{m}$ be the orthonormal basis of $W_{M}$ such that $O_{j}^{i} e_{k}=$ $e_{i} \delta_{j k}-e_{j} \delta_{i k}(M=L, R)$, then we have an orthonormal basis $\left\{e_{j, p}:=e_{j} \otimes e_{p} \mid-l \leq j \leq l\right.$, $-r \leq p \leq r\}$ of $W_{L R}$ (we consider $j$ (resp. $p$ ) does not take 0 if $L$ (resp. $R$ ) is even). By $(j, p)>0$ we mean $j>0$ or $(j=0, p>0)$, and put

$$
\left[\begin{array}{c}
\psi_{j, p} \\
\psi_{-j,-p}
\end{array}\right]:=\left[\begin{array}{c}
\psi^{-j,-p} \\
\psi^{j, p}
\end{array}\right]:=\frac{1}{\sqrt{2}}\left[\begin{array}{cc}
1 & \sqrt{-1} \\
1 & -\sqrt{-1}
\end{array}\right]\left[\begin{array}{c}
e_{j, p} \\
e_{-j,-p}
\end{array}\right]
$$

for $(j, p)>0$ so that $\left\{\psi^{j, p}, \psi_{k . p}\right\}=\delta^{j}{ }_{k} \delta^{p}{ }_{q}(-l \leq j, k \leq l,-r \leq p, q \leq r)$.

$$
W_{L \bar{R}}^{ \pm}:=\bigoplus_{(j, p)>0} \mathbb{C} \psi^{ \pm j, \pm p} \quad \text { (and } e^{0}=\psi^{00}=\psi_{00}:=e_{00} \text { ) }
$$

give a (quasi) isotropic decomposition of $W_{L R}$ :

$$
W_{L R}=W_{L R}^{+} \oplus W_{L R}^{-}\left(\oplus \mathbf{C} e^{0}\right) .
$$

Proposition 3.1.00. For $-l \leq j, k \leq l$ and $-r \leq p, q \leq r$, we have

$$
S \circ \mathrm{L}\left(O_{k}^{j}\right)=\Sigma_{p=-r}^{r}: e_{j, p} e_{k, p}:, \quad S \circ \mathrm{R}\left(O_{q}^{p}\right)=\Sigma_{j=-l}^{l}: e_{j, p} e_{j, q}: .
$$

As for the root vectors $X^{j}{ }_{k}, X^{p}{ }_{q}(X=B, D ; \S 1.4 .6)$,

$$
S \circ \mathrm{L}\left(X_{k}^{j}\right)=\Sigma_{p=-r}^{r}: \psi^{j, p} \psi_{k, p}:, \quad S \circ \mathrm{R}\left({X^{p}}_{q}\right)=\Sigma_{j=-l}^{l}: \psi^{j, p} \psi_{j, q}:
$$

\subsection{The irreducible decompositions}

Now we describe our result in the finite dimensional case, the irreducible decomposition of the $\mathfrak{o}(N)$-spin module $\bigwedge\left(W_{N}^{-}\right)$as a $g_{\mathrm{L}} \oplus \mathfrak{g}_{\mathrm{R}}$-module.

The pair $\left(\mathfrak{g}_{\mathrm{L}}, \mathfrak{g}_{\mathrm{R}}\right)$ is defined by 3.1.1 3, and then $N$ is given in Table 3.2. For each case the space of creation operators $W_{N}^{-}$is respectively chosen as 3.1.1(7), 3.1.2(5), 3.1.3(5), and the representation of $g_{\mathrm{L}} \oplus \mathfrak{g}_{\mathrm{R}}$ on $\bigwedge\left(W_{N}^{-}\right)$is given by Proposition 3.1.AA OO. We fix the Chevalley generators as in $\S 1.4$ so that we can talk about highest weights, which are parametrized by Young diagrams as in $\S 1.4$. Recall Definition 1.3.2(2) of $\mathbb{L}^{\Sigma \ltimes \mathfrak{g}}(\lambda)$.

\section{Theorem 3.2.}

(i). As a $\mathfrak{g}_{\mathrm{L}} \oplus \mathfrak{g}_{\mathrm{R}}$-module $(\S \S 3.1 .1 \sim 3)$, the $\mathfrak{o}(N)$-spin module $\bigwedge\left(W_{N}^{-}\right)$decomposes as follows.

$$
\bigwedge\left(W_{N}^{-}\right) \simeq \bigoplus_{Y \in \mathscr{Y}_{l, r}} \mathbb{L}^{\Sigma_{\mathrm{L}} \times \mathrm{g}_{\mathrm{L}}}\left(\lambda_{\mathrm{L}}(Y) \otimes \mathbb{L}^{\Sigma_{\mathrm{R}} \times \mathrm{g}_{\mathrm{R}}}\left(\lambda_{\mathrm{R}}(Y)\right)\right.
$$

Here l, $r, \Sigma_{\mathrm{L}}, \Sigma_{\mathrm{R}}, \lambda_{\mathrm{L}}, \lambda_{\mathrm{R}}$ are given by Table 3.2 below and $\mathscr{Y}_{l, r}$ denotes the set of all Young diagrams in the $l \times$ r-rectangle (1.4.1.). 
List 3.2(AA-DD) is the complete list of the highest weight vector of each irreducible component with respect to $\mathfrak{g}_{\mathrm{L}} \oplus \mathfrak{g}_{\mathrm{R}}$. All finite dimensional irreducible modules of classical simple Lie algebras appear as an irreducible component of a suitable $\bigwedge\left(W_{N}^{-}\right)$.

(ii) [Hol] In particular, $\bigwedge\left(W_{N}^{-}\right)$admits a $\Sigma_{\mathrm{L}} \times \Sigma_{\mathrm{R}}$-action (1.3.2(4)) and each pair $\left(\mathbb{C}\left[\Sigma_{\mathrm{L}}\right] \ltimes U\left(\mathrm{~g}_{\mathrm{L}}\right), \mathbb{C}\left[\Sigma_{\mathrm{R}}\right] \ltimes U\left(\mathrm{~g}_{\mathrm{R}}\right)\right)$ forms a dual pair (Definition $0.1 \mathrm{~b}$ ) on $\bigwedge\left(W_{N}^{-}\right)$.

Table 3.2

$\begin{array}{ccc}\text { Case } & \mathrm{AA} & \mathrm{CC} \\ \mathrm{g}_{\mathrm{L}}, \mathrm{g}_{\mathrm{R}} & \mathrm{gl}(l), \mathfrak{s l}(r) & \mathfrak{s p}(2 l), \mathfrak{s p}(2 r) \\ \Sigma_{\mathrm{L}}, \Sigma_{\mathrm{R}} & \{i d\},\{i d\} & \{i d\},\{i d\} \\ N & 2 l r & 4 l r \\ \lambda_{\mathrm{L}}(Y), \lambda_{\mathrm{R}}(Y) & (Y,[Y]-l r / 2)^{(*)},{ }^{t} Y & Y, Y^{\dagger}\end{array}$

List of HWV's List AA List CC

$\begin{array}{cccc}\text { Subcases of } \mathrm{OO}^{(* *)} & \mathrm{BB} & \mathrm{DB} & \mathrm{DD} \\ \mathrm{g}_{\mathrm{L}}, \mathrm{g}_{\mathrm{R}} & \mathfrak{o}(2 l+1), \mathfrak{o}(2 r+1) & \mathfrak{o}(2 l), \mathfrak{o}(2 r+1) & \mathfrak{o}(2 l), \mathfrak{o}(2 r) \\ \Sigma_{\mathrm{L}}, \Sigma_{\mathrm{R}} & \{i d\},\{i d\} & \left\langle\sigma_{l-1, l}\right\rangle,\{i d\} & \left\langle\sigma_{l-1, l}\right\rangle,\left\langle\sigma_{r-1, r}\right\rangle \\ N & (2 l+1)(2 r+1) & 2 l(2 r+1) & 4 l r \\ \lambda_{\mathrm{L}}(Y), \lambda_{\mathrm{R}}(Y) & Y+\Lambda_{l}, Y^{\dagger}+\Lambda_{r} & Y+\Lambda_{l}, Y^{\dagger} & Y, Y^{\dagger} \\ \text { List of HWV's } & \text { List BB } & \text { List DB } & \text { List DD }\end{array}$

Notation for Table 3.2. (*): In Case AA, by $\mathbf{L}^{\mathrm{gl}(l)}(\lambda, a)$ we denote a $\mathfrak{g l}(l)=$ $\mathfrak{s l}(l) \oplus \mathbb{C l}_{l}$-module $\mathbb{L}^{\mathfrak{s l}(l)}(\lambda) \otimes \mathbb{C}_{a}$, where for $a \in \mathbb{C}, \mathbb{C}_{a}$ is a one dimensional $\mathbb{C l}_{l}$-module defined by $\mathbb{1}_{l}(1):=a \cdot 1$.

$(* *)$ : In Case OO, $\sigma_{l-1, l}$ is the diagram automorphism of $D_{l}(\S 1.4 .5)$.

\section{List 3.2.AA}

We identify $Y \in \mathscr{Y}_{l, r}$ with the data $\left(\tilde{Y}_{j, p}\right)_{1 \leq j \leq l, 1 \leq p \leq r}$ by

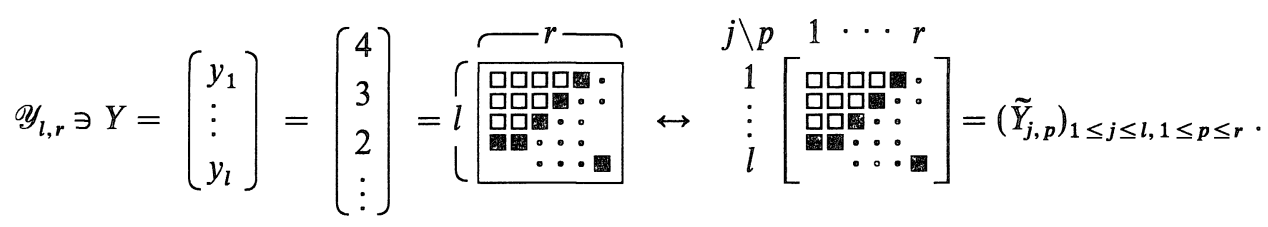

Then the highest weight vector or weight $\left((Y,[Y]-l r / 2),{ }^{t} Y\right)$ with respect to $(\mathfrak{g l}(l), \mathfrak{s l}(r))$ is given by

$$
\left(\vec{\prod}_{(j, p) ; \tilde{Y}_{j, p}=\mathbf{g}} \psi_{j, p}\right) \cdot 1 \in \bigwedge\left(W_{2 l r}^{-}\right)
$$




\section{List 3.2.CC}

We identify $Y \in \mathscr{Y}_{l, r}$ with the data $\left(\tilde{Y}_{j, p}\right)$ as follows:

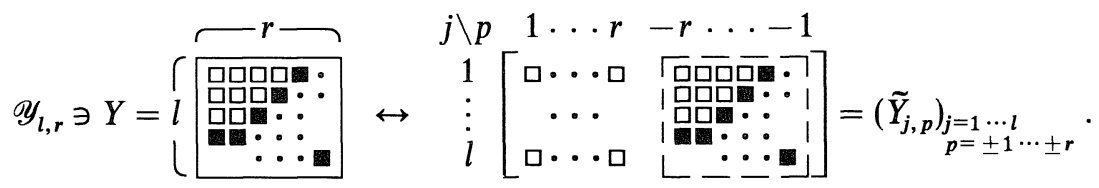

Then the highest weight vector of weight $\left(Y, Y^{\dagger}\right)$ with respect to $(\mathfrak{s p}(2 l), \mathfrak{s p}(2 r))$ is given by $\left(\vec{\prod}_{\tilde{Y}_{\jmath, p}=\mathbf{m}} \psi_{j, p}\right) \cdot 1 \in \bigwedge\left(W_{4 l r}^{-}\right)$.

\section{List 3.2.BB}

Under the identification

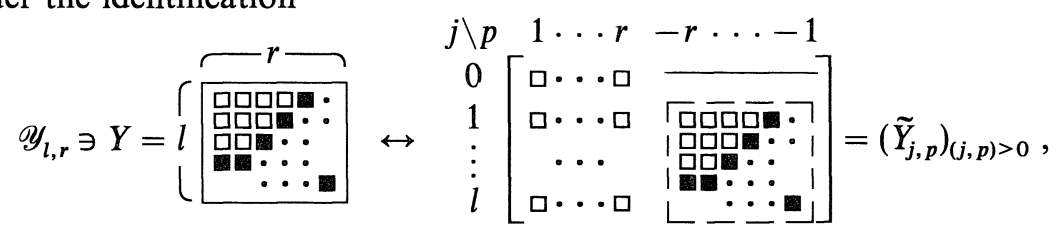

the highest weight vector of the weight $\left(Y+\Lambda_{l}, Y+\Lambda_{r}\right)$ with respect to $(\mathrm{o}(2 l+1)$, $\mathfrak{v}(2 r+1))$ is $\left(\vec{\prod}_{\tilde{Y}_{j, p}=\mathbf{m}} \psi_{j, p}\right) \cdot 1$.

\section{List 3.2.DB ${ }^{(\mathfrak{a})}$}

We make the identification

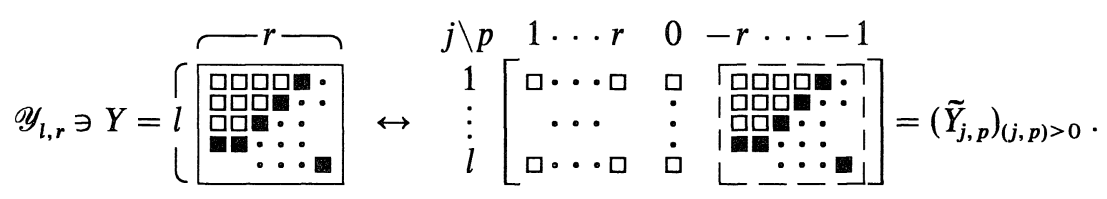

and put $\tilde{\sigma}(\tilde{Y}):=\left(Y_{j, p}+\delta_{j, l} \delta_{Y_{j,-|p|}, \square}\right)_{(j, p)>0}$. Then

$$
|\tilde{Y}\rangle:=\left(\vec{\prod}_{\tilde{Y}_{J, p}= \pm} \psi_{j, p}\right) \cdot 1 \quad \text { and } \quad|\tilde{\sigma}(\tilde{Y})\rangle:=\left(\vec{\prod}_{\tilde{\sigma}(\tilde{Y})_{J, p}=\mathbf{m}} \psi_{j, p}\right) \cdot 1
$$

respectively give the highest weight vector of the weight

$$
\left(Y+\Lambda_{l}, Y^{\dagger}\right) \quad \text { and } \quad\left(\sigma_{l-1, l}\left(Y+\Lambda_{l}\right), Y^{\dagger}\right)
$$

with respect to $(\mathfrak{o}(2 l), \mathfrak{o}(2 r+1))$.

\section{List 3.2. $\mathrm{DD}^{(\text {(t) }}$}

We make the identification

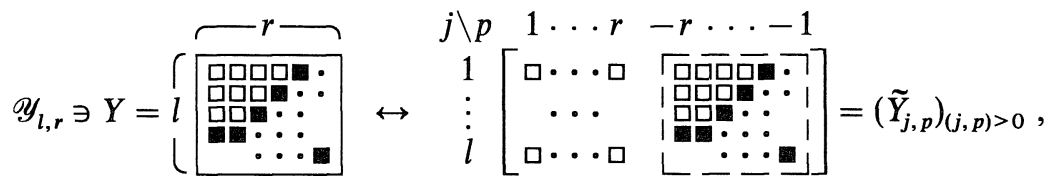


and put

$$
\begin{aligned}
& \left(\tilde{\sigma}^{\mathrm{L}}(\tilde{Y})\right)_{j, p}:=\tilde{\mathbb{Y}}_{j, p}+\delta_{j, l} \delta_{\tilde{Y}_{l,-r}, \square} \delta_{\tilde{Y}_{l, r}, \square} \delta_{\tilde{Y}_{l,-|p|}, \square}, \\
& \left(\tilde{\sigma}^{\mathrm{R}}(\tilde{Y})\right)_{j, p}:=\widetilde{Y}_{j, p}+\operatorname{sgn}(p) \delta_{|p|, r} \delta_{\tilde{Y}_{l, r}, \square} \delta_{\tilde{Y}_{l,-r}, \square} \delta_{\tilde{Y}_{j,-r}, \text { 田 }}
\end{aligned}
$$

Then the vector

$$
\left(\vec{\prod}_{y_{j, p}=\square} \psi_{j, p}\right) \cdot 1 \quad \text { for } \quad\left(y_{j, p}\right)=\tilde{Y}, \tilde{\sigma}^{\mathrm{L}}(\tilde{Y}) \text { and } \tilde{\sigma}^{\mathbb{R}}(\tilde{Y})
$$

respectively gives the highest weight vector of weight

$$
\left(Y, Y^{\dagger}\right), \quad\left(\sigma_{l-1, l}(Y), Y^{\dagger}\right) \quad \text { and } \quad\left(Y, \sigma_{r-1, r}\left(Y^{\dagger}\right)\right) .
$$

Notation for List 3.2. Here and after we denote by $\vec{\prod}$ the product in the exterior algebra which defines a element up to the sign \pm 1 . Moreover,

( 4 ): In these case we identify $\square=0$ and 圆 $=1$, hence $\square+1=$, etc (see also "Notations for List 4.2 ").

Example 3.2. Here we explain the above results in Case AA.

(i) First suppose $r=1$. In this case Theorem 3.2 reads as

$$
\begin{aligned}
\bigwedge\left(W_{2 l}^{-}\right) & \simeq \bigoplus_{p=0}^{l} \mathbb{L}^{\mathfrak{g l}(l)}(Y(p), p-l / 2) \\
& =\bigoplus_{p=0}^{l} \mathbb{L}^{\mathfrak{s l}(l)}\left(\Lambda_{p}\right) \otimes \mathbb{C}_{p-l / 2}
\end{aligned}
$$

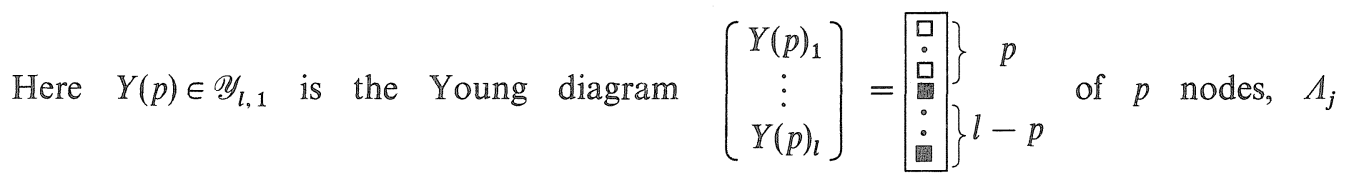
$(1 \leq j \leq l-1)$ is the fundamental weight and we put $\Lambda_{0}=0=\Lambda_{l}$. For each component that corresponds to the Young diagram $Y(p)$, List 3.2.AA gives its highest weight vector as

$$
\vec{\prod}_{j ; Y(p)_{j}=\Xi} \psi_{j} \cdot 1=\psi_{p+1} \cdots \psi_{l} \in \bigwedge^{l-p}\left(W_{2 l}^{-}\right)
$$

We can say, "to give the highest weight vector of each Young diagram Y, just think $Y$ as the Maya diagram ([KNTY], [SN]) of the same form". That is, let us introduce the set of Maya diagrams (of finite size, $l \times r$-component)

$$
\mathfrak{M}_{l, r}:=\left\{\left(m_{j, p}\right)_{\substack{1 \leq j \leq l \\ 1 \leq p \leq r}} \mid m_{j, p}=\text { or } ⿴ 囗 十\right)
$$

and define a pure spinor $|M\rangle$ for each $M \in \mathfrak{M}_{l, r}$ by

$$
\mathfrak{M}_{l, r} \ni \mathbb{M}^{\mathbb{1}}=\left(m_{j, p}\right)_{j, p} \mapsto|M\rangle:=\prod_{m_{j, p}=\boxplus}^{\rightarrow} \psi_{j, p} \in \bigwedge\left(W_{2 l r}^{-}\right) .
$$

Then in this case of $r=1$, we can write the above highest weight vector (2) as

$$
|Y(p)\rangle=\left|\left(Y(p)_{j}\right)_{j=1}^{l}\right\rangle \text {. }
$$


To see that this vector (2) or (5) is actually of weight $\Lambda_{p}$ and is a highest weight vector, use 3.1.1(11) as follows.

$$
\begin{aligned}
S \circ \mathrm{L}\left(E_{j}^{j}\right)\left(\psi_{p+1} \cdots \psi_{l}\right) & =\frac{1}{2} \psi_{p+1} \cdots \psi_{l}+\Sigma_{s=p+1}^{l} \psi_{p+1} \cdots \psi_{s-1}\left(-\psi_{j} \delta^{j}{ }_{s}\right) \psi_{s+1} \cdots \psi_{l} \\
& =\left\{\begin{array}{cc}
\frac{1}{2} \psi_{p+1} \cdots \psi_{l} & (1 \leq j \leq p) \\
\frac{-1}{2} \psi_{p+1} \cdots \psi_{l} & (p<j \leq l),
\end{array}\right.
\end{aligned}
$$

$$
\begin{aligned}
S \circ \mathrm{L}\left(E_{j+1}^{j}\right)\left(\psi_{p+1} \cdots \psi_{l}\right) & =\sum_{s=p+1}^{l} \psi_{p+1} \cdots \psi_{s-1}\left(-\psi_{j+1} \delta^{j}\right) \psi_{s+1} \cdots \psi_{l} \\
& =0 \quad(1 \leq j \leq l-1) .
\end{aligned}
$$

Noting that the $\mathfrak{s l}(l)$-action preserves each $\bigwedge^{s}\left(W_{2 l}^{-}\right)$(Remark 3.1.1), we find that (1) is nothing but the decomposition into the homogeneous parts,

$$
\bigwedge\left(W_{2 l}^{-}\right)=\bigoplus_{p} \bigwedge^{p}\left(W_{2 l}^{-}\right), \quad \bigwedge^{l-p}\left(W_{2 l}^{-}\right) \simeq \mathbb{L}^{\mathrm{sl}(l)}\left(\Lambda_{p}\right) \otimes \mathbf{C}_{p-l / 2} .
$$

Hence together with the $\mathfrak{s l}(l)$-module isomorphisms $\bigwedge\left(W_{2 l}^{-}\right) \simeq \bigwedge\left(\mathbf{C}^{l}\right)^{*}$ (3.1.1(10)) and $\bigwedge^{p}\left(\mathbb{C}^{l}\right)^{*} \simeq \bigwedge^{l-p}\left(\mathbb{C}^{l}\right)$, we also get

$$
\bigwedge\left(\mathbf{C}^{l}\right)=\bigoplus_{p} \bigwedge^{p}\left(\mathbf{C}^{l}\right), \quad \bigwedge^{p}\left(\mathbf{C}^{l}\right) \simeq \mathbb{L}^{\mathrm{sl}(l)}\left(\Lambda_{p}\right) \otimes \mathbf{C}_{p}
$$

This corresponds to the identity of symmetric functions

$$
\prod_{p=1}^{l}\left(1+t x_{p}\right)=\Sigma_{p=0}^{l} t^{p} s_{p}\left(x_{1}, \ldots, x_{l}\right),
$$

where $s_{p}$ stands for the $p$-th fundamental symmetric function.

(ii) Next suppose that $l=3, r=2$. In this case the components are parametrized by the set of Young diagrams

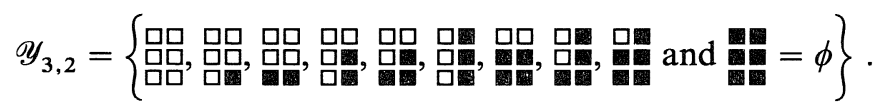

Hence, for example, $\bigwedge^{4}\left(W_{2 l}^{-}\right)(2 l r=12)$ has two components

$$
\mathbf{L}^{\mathrm{gl}(3)}(\because \because \because: 4-3 \cdot 2 / 2) \otimes \mathbb{L}^{\operatorname{sI}(2)}\left(\frac{a}{\square}: \because\right)
$$

and

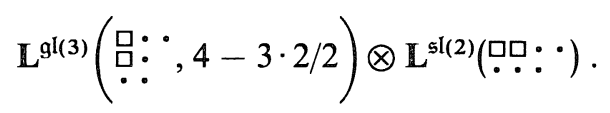

The highest weight vector for the latter component is

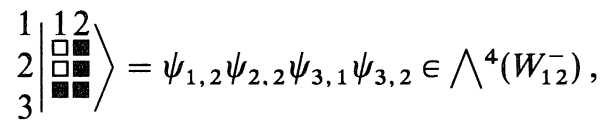

and has weight $0 \cdot \varepsilon_{1}+0 \cdot \varepsilon_{2}+(-1) \cdot \varepsilon_{3} \equiv 0 \Lambda_{1}+1 \Lambda_{2}\left(\operatorname{resp} .(-1 / 2) \cdot \varepsilon_{1}+(-3 / 2) \cdot \varepsilon_{2} \equiv\right.$ 
$\left.2 \Lambda_{1}\right)$ with respect to $\mathfrak{s l}(3)$ (resp. $\left.\mathfrak{s l}(2)\right)$, where $\left\{\varepsilon_{i}\right\}$ is the dual basis to $\left\{E_{i}^{i}\right\}(\S 1.4 .2)$ :

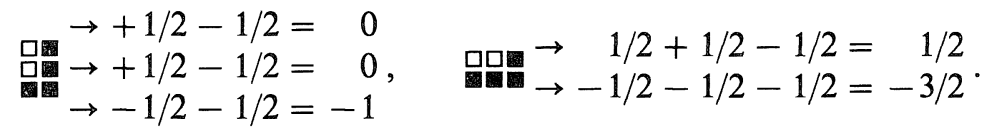

\subsection{Proof of Theorem 3.2}

3.3.1. Except for the Case BB, the proof owes to Lemma 3.3 below. Let $\mathfrak{g}=\mathfrak{n}_{-} \oplus$ $\mathfrak{h} \oplus \pi_{+}$be a simple Lie algebra and its triangular decomposition, and $\mathfrak{g}_{0}$ be the maximal reductive part of a maximal parabolic subalgebra containing $\mathfrak{h}$. We denote the set of positive roots of $(\mathfrak{g}, \mathfrak{h})$ (resp. $\left.\left(\mathfrak{g}_{0}, \mathfrak{h}\right)\right)$ by $\Delta^{+}$(resp. $\left.\Delta_{0}^{+}\right)$, and the Weyl group by $W$ (resp. $W_{0}$ ). Then there is a $\mathbb{Z}_{2}$-gradation $\mathfrak{g}=\mathfrak{g}_{0} \oplus \mathfrak{g}_{1}$, where $\mathfrak{g}_{1}:=\mathfrak{g}_{1}^{+} \oplus \mathfrak{g}_{1}^{-}$and $\mathfrak{g}_{1}{ }^{ \pm}:=$ $\bigoplus_{\alpha \in \Delta^{+} \backslash \Delta_{0}^{+}} \mathfrak{g}_{ \pm \alpha}$, called a Cartan decomposition. In particular $\operatorname{ad}\left(\mathfrak{g}_{0}\right)\left(\mathfrak{g}_{1}\right) \subset \mathfrak{g}_{1}$. Let $K$ be the Killing form of $\mathfrak{g}$. Then $K \mid \mathfrak{g}_{1} \times \mathfrak{g}_{1}$ is non-degenerate and $\operatorname{ad}\left(\mathfrak{g}_{0}\right)$-invariant. Hence we have an inclusion

$$
\mathfrak{g}_{0} \subset \mathfrak{o}\left(\mathfrak{g}_{1}, K\right)
$$

According to this inclusion we restrict the spin representation of $\mathfrak{v}\left(\mathfrak{g}_{1}, K\right)$ to $\mathfrak{g}_{0}$. We fix the isotropic decomposition $\mathfrak{g}_{1}=\mathfrak{g}_{1}^{+} \oplus \mathfrak{g}_{1}^{-}$.

Lemma 3.3. [P] As a $\mathfrak{g}_{0}$-module, the following decomposition holds.

$$
\begin{aligned}
& \bigwedge^{\text {even }}\left(\mathfrak{g}_{1}^{-}\right) \simeq \bigoplus_{w \in W_{1}, \text { sgn } w=1} L^{\mathfrak{g}_{0}}\left(w(\rho)-\rho_{0}\right) \\
& \bigwedge^{\text {odd }}\left(\mathfrak{g}_{1}^{-}\right) \simeq \bigoplus_{w \in W_{1}, \operatorname{sgn} w=-1} \mathbb{L}^{g_{0}}\left(w(\rho)-\rho_{0}\right)
\end{aligned}
$$

Here $W_{1}:=\left\{w \in W \mid w\left(\Delta_{0}^{+}\right) \subset \Delta^{+}\right\}$and $\rho$ (resp. $\left.\rho_{0}\right)$ denotes the half sum of positive roots of $\Delta^{+}\left(\operatorname{resp} . \Delta_{0}^{+}\right)$.

Proof. Because of the linear independence of characters, it suffices to prove the corresponding character identity. Put

$$
\operatorname{ch} \bigwedge^{\text {even }}\left(\mathfrak{g}_{1}^{-}\right)=\Sigma_{\lambda} m_{\lambda}^{+} \operatorname{ch} \mathbb{L}^{g_{0}}(\lambda), \quad \operatorname{ch} \bigwedge^{\text {odd }}\left(g_{1}^{-}\right)=\Sigma_{\lambda} m_{\lambda}^{-} \operatorname{ch} \mathbb{L}^{g_{0}}(\lambda)
$$

with $m_{\lambda}^{ \pm} \in \mathbb{Z}_{\geq 0}$. First we assert

$$
m_{\lambda}^{+}=0 \quad \text { if } \quad m_{\lambda}^{\mp}>0 .
$$

Indeed, if not there is an equality $\lambda=\mu$ of weights for some $\lambda \in P\left(\bigwedge^{\text {even }}\left(g_{1}^{-}\right)\right)$and $\mu \in P\left(\bigwedge^{\text {odd }}\left(\mathfrak{g}_{1}^{-}\right)\right)$. Here $P(\cdot)$ denotes the weight set. Putting $\Delta^{+} \backslash \Delta_{0}^{+}=\left\{\beta_{1}, \ldots, \beta_{n}\right\}$, $\lambda=\mu$ means

$$
\frac{1}{2}\left(s_{1} \beta_{1}+\cdots+s_{n} \beta_{n}\right)=\frac{1}{2}\left(s_{1}^{\prime} \beta_{1}+\cdots+s_{n}^{\prime} \beta_{n}\right)
$$

with $s_{j}, s_{j}^{\prime}= \pm 1$ and $s_{1} \ldots s_{n}=1, s_{1}^{\prime} \ldots s_{n}^{\prime}=-1$. By our assumption for $g_{0}$ there is only one simple root $\alpha_{J} \in \Delta^{+} \backslash \Delta_{0}^{+}$. We have $\Delta^{+} \backslash \Delta_{0}^{+}=\left\{\Sigma n_{j} \alpha_{j} \in \Delta^{+} \mid n_{J}=1\right\}$. Hence expanding $\beta_{j}$ 's in simple roots and comparing the $\alpha_{J}$ coefficient, we have a contradiction and therefore the assertion.

Next we compute the alternative sum of characters, 
(7) $\operatorname{ch} \bigwedge^{\text {even }}\left(\mathfrak{g}_{1}^{-}\right)-\operatorname{ch} \bigwedge^{\text {odd }}\left(\mathfrak{g}_{1}^{-}\right)$

$$
\begin{aligned}
& =\prod_{\alpha \in \Delta^{+} \backslash \Delta_{0}^{+}}\left(1-e^{-\alpha}\right) e^{\rho-\rho_{0}} \quad\left(\bigwedge\left(\mathfrak{g}_{1}^{-}\right) \simeq \bigotimes_{\alpha \in \Delta^{+} \backslash \Delta_{0}^{+}} \bigwedge\left(\mathbf{C g}_{-\alpha}\right) \otimes \mathbf{C} 1 \text { as a h-module }\right) \\
& =\frac{e^{\rho} \prod_{\alpha \in \Delta^{+}}\left(1-e^{-\alpha}\right)}{e^{\rho_{0}} \prod_{\alpha \in \Delta_{0}^{+}}\left(1-e^{-\alpha}\right)} \\
& \left.=\frac{\Sigma_{w \in W}(\operatorname{sgn} w) e^{w(\rho)-\rho_{0}}}{\prod_{\alpha \in \Delta_{0}^{+}}\left(1-e^{-\alpha}\right)} \quad \text { Weyl denominator formula }\right) \\
& =\Sigma_{w \in W_{1}}(\operatorname{sgn} w) \frac{\Sigma_{w^{\prime} \in W_{0}}\left(\operatorname{sgn} w^{\prime}\right) e^{w^{\prime}\left(w(\rho)-\rho_{0}+\rho_{0}\right)-\rho_{0}}}{\prod_{\alpha \in \Delta_{0}^{+}}\left(1-e^{-\alpha}\right)} \quad\left(W=W_{0} W_{1}\right) \\
& =\Sigma_{w \in W_{1}}(\operatorname{sgn} w) \operatorname{ch~} \mathbb{L}^{g_{0}}\left(w(\rho)-\rho_{0}\right) \quad \text { (Weyl character formula). }
\end{aligned}
$$

In the last equality we used the fact that $w(\rho)-\rho_{0}$ is dominant integral with respect to $\mathrm{g}_{0}$ for each $w \in W_{1}$. If we put this

$$
=\Sigma_{\lambda} n_{\lambda}^{+} \operatorname{ch} \mathbb{L}^{g_{0}}(\lambda)-\Sigma_{\lambda} n_{\lambda}^{-} \operatorname{ch} \mathbf{L}^{g_{0}}(\lambda)
$$

with $n_{\lambda}^{+} \geq 0$, now we have only to show $m_{\lambda}^{\frac{t}{\lambda}}=n_{\lambda}^{\frac{t}{\lambda}}$. Note that $n_{\lambda}^{+}=0$ if $n_{\lambda}^{\mp}>0$. Then from this property and (5), $m_{\lambda}^{+}=n_{\lambda}^{ \pm}$follows.

Proof of Theorem 3.2, Cases AA, CC, DB and DD. We put

$$
\mathfrak{g}:= \begin{cases}\mathfrak{s l}(l+r) & : \text { in Case AA, } \\ \mathfrak{s p}(2 l+2 r) & : \text { in Case CC } \\ \mathfrak{o}(L+R) & : \text { in Case OO }\end{cases}
$$

Then in these cases we observe that the inclusion $\mathfrak{g}_{L} \oplus \mathfrak{g}_{R} \subset \mathfrak{v}(N)$ in 3.1 coincides with what introduced in (1): if we take $\mathrm{g}_{0}=\mathrm{g}_{L} \oplus \mathrm{g}_{R}$, then $\mathrm{g}_{1} \simeq \mathbf{C}^{N} \simeq W_{N}$. Hence thanks to Lemma 3.3, we only have to describe the coset $W_{1}$ explicitly in terms of Young diagrams to get the irreducible decomposition. We can consult [JM, Proposition 1.1] for this procedure; the idea is to use the following bijections.

Fact 3.3.1. We have the following commutative diagram of bijections of sets.

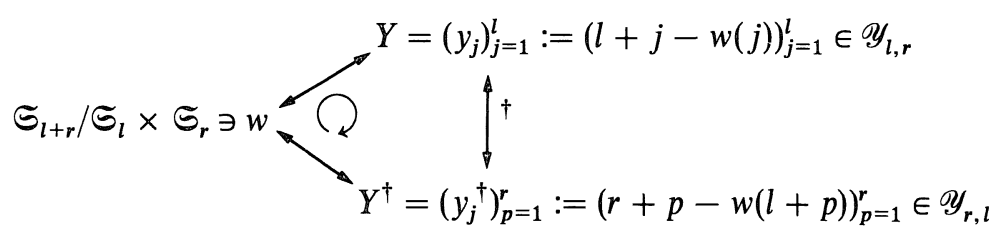

Here the representative $w$ is assumed to satisfy the conditions

$$
w(1)<w(2)<\cdots<w(l) \quad \text { and } \quad w(l+1)<\cdots<w(l+r) .
$$

Under this bijection it holds that $\operatorname{sgn}(w)=(-1)^{\left[Y^{\dagger}\right]}$. As a corollary, it holds that $\# \mathscr{Y}_{l, r}=$ $\# \mathscr{Y}_{r, l}=\frac{(l+r) !}{l ! r !}$.

Together with this statement and the description of the Weyl groups given in $\S 1.4$ 
we obtain the desired description of the coset $W_{1}$, and this is what we can find in [JM]. The statement for the highest weight vectors can be verified directly.

爽

Remark 3.3.1. (ii) The above correspondance (9) is nothing but the graphical execution of the subtraction $w(\rho)-\rho_{0}$ for Case CC.

(ii) In the case $\mathrm{AA}$, Lemma 3.3 specializes to the identity

$$
\prod_{\substack{1 \leq j \leq l \\ 1 \leq p \leq r}}\left(1+w_{j} z_{p}\right)=\Sigma_{Y \in \mathscr{V}_{l, r}} \chi_{Y}^{\mathrm{gl}(l)}\left(w_{1}, \ldots, w_{l}\right) \chi_{t_{Y}}^{\mathrm{gl}(r)}\left(z_{1}, \ldots, z_{r}\right)
$$

of the gl-characters $\chi_{Y}$, which is also appear in [Mac, p. 35(4.3')].

(iii) Lemma 3.3 is a result of Parthasarathy [P, Lemma 2.2]. This type of application of the character formula is independently noticed in [JM], which is our startpoint of the present work. [Has].

(iv) Theorem 3.2(AA, CC, DB, DD) can be elementarily shown by the Laplace expansion formula of determinant (of course with the knowledge of character formula), as we will do in the next paragraph for the case $\mathbb{B B}$.

3.3.2. Proof of Theorem 3.2, Case $\mathbb{B B B}$. Since $\mathfrak{g}_{0}=\mathfrak{v}(2 l+1) \oplus \mathfrak{v}(2 r+1)$ does not contain a Cartan subalgebra of $\mathfrak{g}=\mathfrak{o}(2 l+2 r+2)$, Lemma 3.3 does not work in this case. But then instead of 3.3.1(7) we consider

$$
N_{\Lambda_{l+r}^{\mathrm{o}}(2 l+2 r+1)} / D^{\mathrm{o}(2 l+1)} D^{\mathrm{o}(2 r+1)},
$$

where $D^{\circ}$ is the Weyl denominator and $N_{\lambda}^{*}:=D^{\circ} \cdot \operatorname{ch} \mathbb{L}^{\circ}(\lambda)$, to deduce our result. Namely, we have

(2) $\operatorname{Trace}_{\bigwedge\left(W^{-}(2 l+1)(2 r+1)\right.} \exp \left(\sum_{j=1}^{l} \eta_{j} B_{j}^{j}+\sum_{p=1}^{r} \zeta_{p} B_{p}^{p}\right)$

$$
\begin{aligned}
& \times D^{\mathfrak{o}(2 l+1)}\left(w_{1}, \ldots, w_{l}\right) D^{\mathfrak{o}(2 r+1)}\left(z_{1}, \ldots, z_{r}\right) \\
& =\prod_{j=1}^{l}\left(w_{j}^{1 / 2}+w_{j}^{-1 / 2}\right) \cdot \prod_{p=1}^{r}\left(z_{p}^{1 / 2}+z_{p}^{-1 / 2}\right) \cdot \prod_{j=1}^{l} \prod_{p=1}^{r}\left(w_{j}+z_{p}{ }^{-1}\right)\left(1+w_{j}^{-1} z_{p}\right) \\
& \times \prod_{j=1}^{l}\left(w_{j}^{1 / 2}-w_{j}^{-1 / 2}\right) \cdot \prod_{1 \leq j<k \leq l}\left(w_{j}-w_{k}\right)\left(1-w_{j}^{-1} w_{k}{ }^{-1}\right) \\
& \times \prod_{p=1}^{r}\left(z_{p}^{1 / 2}-z_{p}{ }^{-1 / 2}\right) \cdot \prod_{1 \leq p<q \leq r}\left(z_{p}-z_{q}\right)\left(1-z_{p}{ }^{-1} z_{q}{ }^{-1}\right) \\
& =(-1)^{l(l+1) / 2} \cdot N_{\Lambda_{l+r}}^{\mathrm{o}(2 l+2 r+1)}\left(w_{1}, \ldots, w_{l},-z_{1}{ }^{-1}, \ldots,-z_{r}^{-1}\right)
\end{aligned}
$$

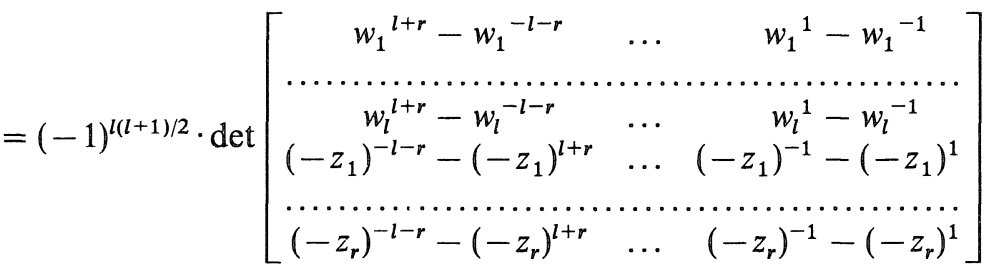

$$
\begin{aligned}
& =\Sigma_{Y \in \mathscr{Y}_{l, r}}(-1)^{l(l+1) / 2+[Y \dagger]} \\
& \times \operatorname{det}\left[w_{j}^{r+1-k+y_{k}}-w_{j}^{-r-1+k-y_{k}}\right] \operatorname{det}\left[\left(-z_{p}\right)^{-l-1+q-y_{q}^{\dagger}}-\left(-z_{p}\right)^{l+1-q+y_{q}^{\dagger}}\right] \\
& =\Sigma_{Y \in \mathscr{Y}_{l, r}}(-1)^{l(l+1) / 2+\left[Y^{\dagger}\right]+\sum_{q}\left(l+1-q+y_{q}^{\dagger}\right)} \\
& \times \operatorname{det}\left[w_{j}^{r+1-k+y_{k}}-w_{j}^{-r-1+k-y_{k}}\right] \operatorname{det}\left[z_{p}^{-l-1+q-y_{q}^{\dagger}}-z_{p}^{l+1-q+y_{q}^{\dagger}}\right] \\
& =\Sigma_{Y \in \mathscr{Y}_{l, r}} N_{Y+\Lambda_{l}}^{\mathrm{o}(2 l+1)}\left(w_{1}, \ldots, w_{l}\right) \cdot N_{Y^{f}+\Lambda_{r}}^{\mathrm{o}(2 r+1)}\left(z_{1}, \ldots, z_{r}\right)
\end{aligned}
$$


for $\eta_{j}, \zeta_{p} \in \mathbb{C}$, where $w_{j}=\exp \eta_{j}$ and $z_{p}=\exp \zeta_{p}$. Here we used the Laplace expansion formula of determinant in the following form, which is a consequence of Fact 3.1.1.

(3) $\operatorname{det}\left[x_{j, k}\right]_{1 \leq j, k \leq l+r}=\Sigma_{Y \in \mathscr{Y}_{l, r}}(-1)^{\left[Y^{\dagger}\right]} \operatorname{det}\left[x_{j, k+r-y_{k}}\right]_{1 \leq j, k \leq l} \operatorname{det}\left[x_{l+p, l+q-y_{k}^{\dagger}}\right]_{1 \leq p, q \leq r}$.

Now dividing both sides by $D^{\mathfrak{o}(2 l+1)} D^{\mathfrak{o}(2 r+1)}$ we get the desired identity

$$
\begin{aligned}
& \operatorname{Trace}_{\Lambda\left(W^{-}(2 l+1)(2 r+1)\right)} \exp \left(\sum_{j=1}^{l} \eta_{j} B_{j}^{j}+\sum_{p=1}^{r} \zeta_{p} B_{p}^{p}\right) \\
& \quad=\Sigma_{Y \in \mathscr{Y}_{l, r}} \operatorname{ch}_{\mathbf{L}\left(Y+\Lambda_{l}\right)}^{\mathrm{o}(2 l+1)}\left(w_{1}, \ldots, w_{l}\right) \cdot \operatorname{ch}_{\mathbf{L}\left(Y^{+}+\Lambda_{r}\right)}^{\mathrm{o}(2 r+1)}\left(z_{1}, \ldots, z_{r}\right) .
\end{aligned}
$$

\section{§4. Dual Pairs of Classical Affine Lie Algebras}

4.0. This section is the main part of this paper. In 4.1 we give a generalization of Yamanaka's result ([Ya]) by using central charge calculations. In 4.2 we describe the main result for classical nontwisted affine Lie algebras. We prove them in $4.3 \sim 4.4$.

\subsection{Definition of representations}

We retain the notation in Section 3. The inclusions

$$
\mathrm{L}: \mathfrak{g}_{\mathrm{L}} \rightarrow \mathfrak{o}\left(W_{N}\right) \quad \text { and } \quad \mathrm{R}: \mathfrak{g}_{\mathrm{R}} \rightarrow \mathfrak{o}\left(W_{N}\right)
$$

$(3.1 .1 \sim 3,(1)(2))$ induce inclusions

$$
\mathrm{L}^{\wedge}: \mathfrak{g}_{\hat{\mathrm{L}}}^{\wedge} \rightarrow \mathfrak{p}\left(W_{N}\right)^{\wedge} \quad \text { and } \quad \mathrm{R}^{\wedge}: \mathfrak{g}_{\mathrm{R}}^{\wedge} \rightarrow \mathfrak{v}\left(W_{N}\right)^{\wedge}
$$

respectively defined by the formulae

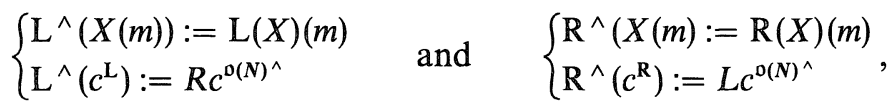

where $c^{\mathfrak{o}(N)^{\wedge}}\left(\right.$ resp. $\left.c^{\mathbf{L}}, c^{\mathbf{R}}\right)$ denotes the canonical central element of $\mathfrak{o}(N)^{\wedge}\left(\right.$ resp. $\left.\mathfrak{g}_{\mathbf{L}}^{\wedge}, \mathfrak{g}_{\mathbf{R}}\right)$ and $R$ (resp. $L$ ) is the positive integer given in Table 4.2. (The number $R$, as well as $L$, is the ratio $R:=B^{\mathrm{o}(N)}(\mathrm{L}(x), \mathrm{L}(x)) / B^{\mathrm{g}_{\mathrm{L}}}(x, x)$ which does not depend on $x \in \mathrm{g}_{\mathrm{L}}, x \neq 0$, where $B^{\mathfrak{g}}$ denotes the normalized bilinear form of $g(1.4)$.) For $h=0$ or $1 / 2$, let $W_{N}^{\mathbf{Z}+h,-}$ be the space of creation operators given by 3.1.1(7), 3.1.2(5), 3.1.3(5) and 2.4(1 4), and let $S^{\wedge}=S^{\mathbb{Z}+h}$ be the spin representation of $\mathfrak{o}\left(W_{N}\right)^{\wedge}$ on $\bigwedge\left(W_{N}^{\mathbb{Z}+h,-}\right)(2.4(7))$.

Then our interest is in the representations $S^{\wedge} \circ \mathrm{L}^{\wedge}$ and $S^{\wedge} \circ \mathrm{R}^{\wedge}$ of $\mathfrak{g}_{\mathrm{L}}^{\wedge}$ and $\mathfrak{g}_{\hat{R}}$, respectively.

The representations $S \circ \mathrm{L}, S \circ \mathrm{R}$ of $\mathfrak{g}_{\mathrm{L}}, \mathfrak{g}_{\mathrm{R}}$ are given by the formulae in Propositions 3.1.AA $\sim \mathrm{OO}$, which are of the form

$$
S \circ \mathrm{L}(X)=\Sigma: a b: \quad \text { and } \quad S \circ \mathrm{R}(X)=\Sigma: a^{\prime} b^{\prime}:,
$$

with $a, b ; a^{\prime}, b^{\prime} \in W_{N}$. Then the representations $S^{\wedge} \circ \mathrm{L}^{\wedge}, S^{\wedge} \circ \mathrm{R}^{\wedge}$ of $\mathfrak{g}_{\mathbf{L}}^{\wedge}, \mathfrak{g}_{\mathbf{R}}^{\wedge}$ are given by the following formulae 
and

$$
\left\{\begin{array}{l}
S^{\wedge} \circ \mathbb{L}^{\wedge}(X(m))=\sum \Sigma_{\mu+v=m}: a(\mu) b(v): \\
S^{\wedge} \circ \mathrm{L}^{\wedge}\left(c^{\mathbf{L}}\right)=R \cdot i d
\end{array}\right.
$$

$$
\left\{\begin{array}{l}
S^{\wedge} \circ \mathbb{R}^{\wedge}(X(m))=\sum \Sigma_{\mu+v=m}: a^{\prime}(\mu) b^{\prime}(v):, \\
S^{\wedge} \circ \mathbb{R}^{\wedge}\left(c^{\mathbf{R}}\right)=L \cdot i d
\end{array}\right.
$$

in End $\bigwedge\left(W_{N}^{\mathbf{Z}+h,-}\right)$. For example, Proposition 3.1.AA yields

$$
S^{\wedge} \circ \mathbb{L}^{\wedge}\left(E_{j}^{i}(m)\right)=\sum_{p=1}^{r} \sum_{\mu+\nu=m}: \psi^{i, p}(\mu) \psi_{j, p}(v):
$$

for the representation $S^{\wedge} \circ \mathbb{L}^{\wedge}$ of $\operatorname{gl}(l)^{\wedge}$. Here $\mu$ and $v$ run over $\mathbb{Z}+h(h=0,1 / 2)$ and we followed the notation in $\S 2.4$.

Both $S^{\wedge} \circ \mathbb{L}^{\wedge}$ and $S^{\wedge} \circ \mathbb{R}^{\wedge}$ define Virasoro module structures on $\bigwedge\left(W_{N}^{\mathbb{Z}+h,-}\right)$ by 1.2.2(3). We observe

Proposition 4.1. (i) [Ya]. Let $D^{\mathrm{L}}(\mathrm{m})$ and $D^{\mathrm{R}}(\mathrm{m})$ are Virasoro operators obtained by $S^{\wedge} \circ \mathrm{L}^{\wedge}$ and $S^{\wedge} \circ \mathrm{R}^{\wedge}$ through the Segal-Sugawara construction (1.2(8)). Then we have

$$
D^{\mathbf{L}}(m)+D^{\mathbf{R}}(m)=D^{\mathrm{o}(N)^{\wedge}}(m)=D^{\text {Clif }}(m) \in \text { End } \bigwedge\left(W_{N}^{\mathrm{Z}+h,-}\right)
$$

for all $m \in \mathbb{Z}$.

(ii) As a $\mathrm{g}_{\mathrm{L}}^{\wedge} \oplus \mathfrak{g}_{\mathrm{R}} \hat{-}^{-m o d u l e}, \bigwedge\left(W_{N}^{\mathbb{Z}+h,-}\right)$ decomposes into finitely many irreducible components.

Proof. In view of Proposition 1.2, this follows from the central charge identity $z^{\mathfrak{g}_{\mathbf{L}}}+z^{\mathfrak{g}_{\mathfrak{R}}^{\wedge}}=N / 2$, or

$$
\frac{R \operatorname{dim} \mathfrak{g}_{\mathrm{L}}}{R+g\left(\mathfrak{g}_{\mathrm{L}}\right)}+\frac{L \operatorname{dim} \mathfrak{g}_{\mathrm{R}}}{L+g\left(\mathfrak{g}_{\mathrm{R}}\right)}=N / 2
$$

for each case $\left(\right.$ in case of $\left(\mathfrak{g}_{\mathrm{L}}^{\wedge}, \mathfrak{g}_{\hat{R}}^{\wedge}\right)=\left(\mathfrak{g l}(l)^{\wedge}, \mathfrak{s l}(r)^{\wedge}\right)$, read the left hand side as $1+$ $\left.\frac{r\left(l^{2}-1\right)}{r+l}+\frac{l\left(r^{2}-1\right)}{l+r}\right)$.

Remark 4.1. The central charge identity (8) plays an essential role in the above. The identities (7) of operators can be also verified by a direct computation, which will be found in [Ya] for the pairs $\left(\mathfrak{g}_{\hat{L}}^{\wedge}, \mathfrak{g}_{\mathbb{R}}^{\wedge}\right)=\left(\mathfrak{s p}(2 l)^{\wedge}, \mathfrak{s p}(2 r)^{\wedge}\right)$ or $\left(\mathfrak{g l}(l)^{\wedge}, \mathfrak{s l}(r)^{\wedge}\right)$. To show (7) Yamanaka calculate there the Virasoro operators with using the Wick's theorem and the operator product expansion.

\subsection{The irreducible decompositions}

Now we are in a position to describe the irreducible decompositions of the $\mathfrak{o}(N)^{\wedge}$ spin module $\bigwedge\left(W_{N}^{\mathbb{Z}+h,-}\right)$, viewed as a $\mathfrak{g}_{\mathfrak{L}}^{\wedge} \oplus \mathfrak{g}_{\mathbf{R}}$-module through $4.1(4 \sim 5)$. Using the notation in $\S 3.1$, we fix the space $W_{N}^{\mathbb{Z}+h,-}(h=0$ : Ramond sector or $h=1 / 2$ : NeneuSchwarz sector) of creation operators as stated in $\S 4.1$ and the triangular decompositions of $\mathfrak{g}_{\hat{L}}$ and $\mathfrak{g}_{\hat{R}}$ by 1.2.1(4). Recall the definition of $\mathbb{L}^{\Sigma \ltimes \mathfrak{g}}(\lambda, l)(1.2 .3(1 \sim 2)$, 1.3.2(2), 1.3.4(4 6)). 


\section{Theorem 4.2. (Main Theorem.)}

As a $\mathfrak{g}_{\mathrm{L}}^{\wedge} \oplus \mathfrak{g}_{\mathrm{R}}^{\wedge}$-module, the $\mathfrak{o}(N)^{\wedge}$-spin module $\bigwedge\left(W_{N}^{\mathbf{Z}+h,-}\right)$ decomposes as follows:

$$
\bigwedge\left(W_{N}^{\mathbf{Z}+h,-}\right) \simeq \bigoplus_{Y} \mathbf{L}^{\Sigma_{\mathbf{L}}^{\wedge} \times \mathfrak{g}_{\mathbf{L}}}\left(\hat{\lambda}_{\mathbf{L}}(Y) ; R\right) \otimes \mathbf{L}^{\Sigma_{\hat{R}}^{\hat{R}} \times \mathfrak{g}_{\mathbf{R}}}\left(\hat{\lambda}_{\mathbf{R}}(Y) ; L\right) .
$$

Here $h=0$ or $1 / 2$, and $\Sigma_{\hat{L}}^{\wedge}, \Sigma_{\mathbf{R}}, \hat{\lambda}_{L}, \hat{\lambda}_{R}, R, L$ and where $Y$ runs over are given by Table 4.2. The complete list of the highest weight vectors with respect to $\mathfrak{g}_{\hat{L}} \oplus \mathfrak{g}_{\hat{R}}$ is given by List 4.2.

In particular, $\bigwedge\left(W_{N}^{\mathbf{Z}+h,-}\right)$ admits an action of $\Sigma_{\mathbf{L}}^{\wedge} \times \Sigma_{\hat{R}}^{\wedge}(1.3 .2(4))$ and each pair $\left(\Sigma_{\mathbf{L}}^{\wedge} \ltimes \mathfrak{g}_{\mathbf{L}}^{\wedge}, \Sigma_{\mathbf{R}}^{\wedge} \ltimes \mathfrak{g}_{\mathbf{R}}^{\wedge}\right)$ forms a dual pair.

\section{Table 4.2.}

\begin{tabular}{|c|c|c|}
\hline Case & $\hat{A} \hat{A}$ & $\hat{\mathrm{C}} \hat{\mathrm{C}}$ \\
\hline $\mathfrak{g}_{\mathrm{L}}^{\wedge}, \mathfrak{g}_{\hat{R}} \hat{~}$ & $\operatorname{gl}\left(()^{\wedge}, \mathfrak{s l}(r)^{\wedge}\right.$ & $\mathfrak{s p}(2 l)^{\wedge}, \mathfrak{s p}(2 r)^{\prime}$ \\
\hline$\Sigma_{\hat{L}}^{\wedge}, \Sigma_{\hat{R}}$ & $\left\langle\sigma_{c y c}^{\#}\right\rangle,\{i d\}$ & $\{i d\},\{i d\}$ \\
\hline$R, L$ & $r, l$ & $r, l$ \\
\hline$N$ & $2 l r$ & $4 l r$ \\
\hline$\hat{\lambda}_{\mathbf{L}}(Y), \hat{\lambda}_{\mathbf{R}}(Y)\left\{\begin{array}{l}h=0 \\
h=\frac{1}{2}\end{array}\right.$ & $\left\{\begin{array}{l}(Y,[Y]-\operatorname{lr} / 2),{ }^{t} Y \\
(Y,[Y]),{ }^{t} Y\end{array}\right.$ & $\left\{\begin{array}{l}Y, Y^{\dagger} \\
Y,{ }^{t} Y\end{array}\right.$ \\
\hline$Y$ runs over & $Y \in \mathscr{Y}_{l, r-1}$ & $Y \in \mathscr{Y}_{l, r}$ \\
\hline List of HWV's & List $\hat{A} \hat{A}$ & List $\hat{C} \widehat{C}$ \\
\hline
\end{tabular}

\begin{tabular}{|c|c|c|c|}
\hline Subcases of ÔÔ & $\hat{\mathbf{B}} \hat{\mathbf{B}}$ & $\hat{\mathrm{D}} \hat{\mathrm{B}}$ & D̂D̂ \\
\hline $\mathfrak{g}_{\mathbf{L}}^{\hat{L}}, \mathfrak{g}_{\hat{R}}$ & $\mathfrak{o}(2 l+1)^{\wedge}, \mathfrak{o}(2 r+1)^{\wedge}$ & $\mathfrak{o}(2 l)^{\wedge}, \mathfrak{o}(2 r+1)^{\wedge}$ & $\mathfrak{o}(2 l)^{\wedge}, \mathfrak{o}(2 r)^{\wedge}$ \\
\hline$\Sigma_{\mathbf{L}}^{\hat{n}}, \Sigma_{\hat{R}}$ & $\left\langle\sigma_{01}\right\rangle,\left\langle\sigma_{01}\right\rangle$ & $\left\langle\sigma_{01}, \sigma_{l-1, l}\right\rangle,\left\langle\sigma_{01}\right\rangle$ & $\left\langle\sigma_{01}, \sigma_{l-1, l}\right\rangle,\left\langle\sigma_{01}, \sigma_{r-1, r}\right\rangle$ \\
\hline$R, L$ & $2 r+1,2 l+1$ & $2 r, 2 l+1$ & $2 r, 2 l$ \\
\hline$N$ & $(2 l+1)(2 r+1)$ & $2 l(2 r+1)$ & $4 l r$ \\
\hline$\hat{\lambda}_{\mathrm{L}}(Y), \hat{\lambda}_{\mathrm{R}}(Y)\left\{\begin{array}{l}h=0 \\
\end{array}\right.$ & $\left\{\begin{array}{l}Y+\Lambda_{l}, Y^{\dagger}+\Lambda_{r} \\
t_{t}\end{array}\right.$ & $\left\{\begin{array}{l}Y+\Lambda_{l}, Y^{\dagger} \\
y_{t}\end{array}\right.$ & $\left\{\begin{array}{l}Y, Y^{\dagger} \\
Y_{t}{ }^{\prime}\end{array}\right.$ \\
\hline$Y$ runs over & $Y \in \mathscr{Y}_{l, r}$ & $Y \in \mathscr{Y}_{l, r}$ & $Y \in \mathscr{Y}_{l, r}$ \\
\hline List of HWV's & List $\hat{B} \hat{B}$ & List $\hat{D} \hat{B}$ & List $\hat{D} \hat{D}$ \\
\hline
\end{tabular}

Notations in Table 4.2. For $\sigma_{c y c}^{\#}$ and $(Y, a)$ in Case $\hat{A} \hat{A}$, see 1.3.4(1 6). $\sigma_{01}$, $\sigma_{l-1, l}: 1.5 .3 \sim 5(4) . \quad \Lambda_{l}: 1.4 .3 \sim 5(3.1) . \quad \mathscr{Y}_{l, r},[Y], Y^{\dagger},{ }^{t} Y: 1.4 .1 . \quad$ Identification of Young diagrams with weights: $1.4 .2 \sim 5(3.3)$.

\section{List 4.2.ÂÂA}

Let $\varepsilon_{j, p}(1 \leq j \leq l, 1 \leq p \leq r)$ be the orthonormal basis of the lattice $\mathbf{Z}^{l r}=$ $\bigoplus_{j, p} \mathbf{Z}_{\varepsilon_{j, p}} \subset\left(\mathfrak{h}^{\mathfrak{o}(2 l r)}\right)^{*}$ that are dual to $D^{j+l p}{ }_{j+l p} \in \mathfrak{h}^{\mathfrak{D}(2 l r)}(1.4 .5(2))$, so that we have 


$$
\varepsilon_{j, p}\left(\mathrm{~L}\left(E_{i}^{i}\right)\right)=\delta_{j}^{i} \quad \text { and } \quad \varepsilon_{j, p}\left(\mathbb{R}\left(E_{q}^{q}\right)\right)=\delta_{p}^{q} .
$$

Put $\check{h}:=1 / 2-h$. For each element $\gamma=\Sigma_{1 \leq j \leq l, 1 \leq p \leq r} \gamma_{j, p} \varepsilon_{j, p} \in \mathbb{Z}^{l r}+\check{h} \mathbb{1}^{*}$, where $\mathbb{1}^{*}:=\Sigma_{j, p} \varepsilon_{j, p}$, we can assign a vector $|\gamma\rangle \in \bigwedge\left(W_{2 l r}^{\mathbb{Z}+h,-}\right)$ defined by 8.1(5). Then $\operatorname{gl}(l)^{\wedge} \oplus \mathfrak{s l}(r)^{\wedge}$-highest weight vectors are spanned by the set

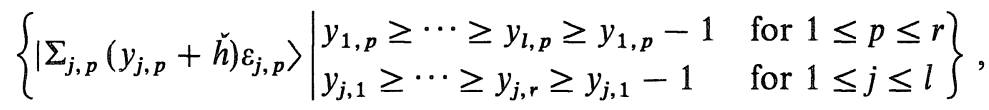

and the weight of $\left|y+\check{h} \mathbb{1}^{*}\right\rangle=\left|\Sigma_{j, p}\left(y_{j, p}+\check{h}\right) \varepsilon_{j, p}\right\rangle$ is given by

$$
\left(\left(\sum_{j=1}^{l}\left(\sum_{p=1}^{r} y_{j, p}+\check{h}\right) \varepsilon_{j}\right),\left.\left(\sum_{p=1}^{r}\left(\sum_{j=1}^{l} y_{j, p}+\check{h}\right) \varepsilon_{p}\right)\right|_{\mathfrak{h}}\right)
$$

for $\left(\operatorname{gl}(l)^{\wedge}, \mathfrak{s l}(r)^{\wedge}\right)$, respectively (see also Example 4.2).

\section{List 4.2. $\widehat{\mathbb{C}} \widehat{\mathbb{C}}$}

$(\boldsymbol{h}=\mathbf{0})$ : Under the identification

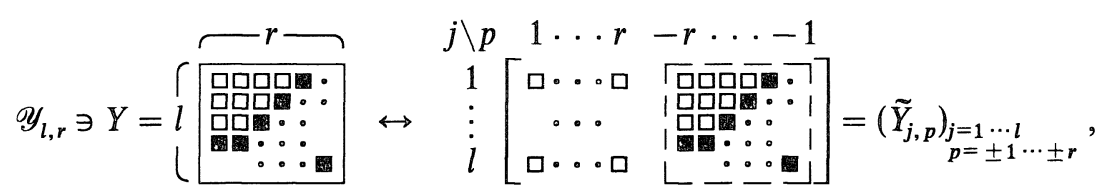

the highest weight vector of weight $\left(Y, Y^{\dagger}\right)$ is given by

$$
\left(\vec{\prod}_{\tilde{Y}_{J, p}=\operatorname{mos}} \psi_{j, p}(0)\right) \cdot 1
$$

$\left(h=\frac{1}{2}\right):$ Under the identification

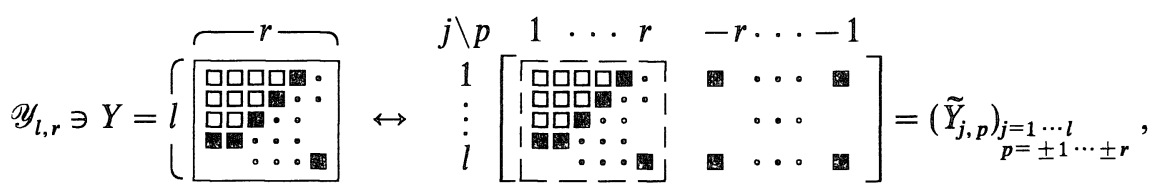

the highest weight vector of weight $\left(Y,{ }^{t} Y\right)$ is given by

$$
\left(\vec{\prod}_{\tilde{Y}_{J, p}=\square} \psi^{j, p}\left(-\frac{1}{2}\right)\right) \cdot 1
$$

\section{List 4.2. $\widehat{B} \hat{B}^{*}$}

$(h=0)^{* *}: \quad$ Identify $Y \in \mathscr{Y}_{l, r}$ with $\tilde{Y} \in \operatorname{Mat}(2 l+1,2 r+1)$ as

$$
\begin{aligned}
& j \backslash p \quad 1 \quad \cdots \quad r \quad 0 \quad-r \ldots-1
\end{aligned}
$$

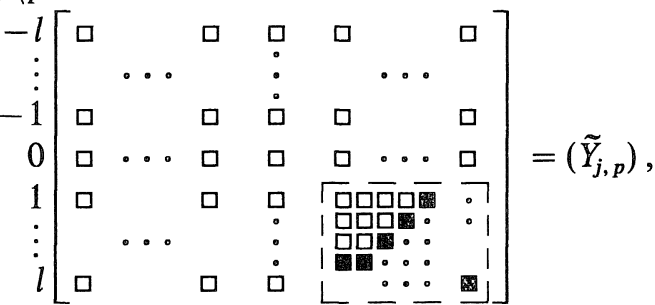


and define $\tilde{\sigma}_{01}^{\mathrm{L}}(\tilde{Y}), \tilde{\sigma}_{01}^{\mathrm{R}}(\tilde{Y}) \in \operatorname{Mat}(2 l+1,2 r+1)$ by

$$
\begin{aligned}
& \left(\tilde{\sigma}_{01}^{\mathrm{L}}(\tilde{Y})\right)_{j, p}:=\tilde{Y}_{j, p}+\operatorname{sgn}(j) \delta_{|j|, 1} \delta_{\tilde{Y}_{1, p}, \text { ⿴囗十 }}, \\
& \left(\tilde{\sigma}_{01}^{\mathrm{R}}(\tilde{Y})\right)_{j, p}:=\tilde{Y}_{j, p}+\delta_{p,-1} \delta_{\tilde{Y}_{|j|, 1}, \square} .
\end{aligned}
$$

Then the vector $* *\left(\vec{\prod}_{y_{j, p}=\text { 回 }} \psi_{j, p}\left(\mu_{0}\right)\right) \cdot 1$ for each of the matrices

$$
\left(y_{j, p}\right)=\tilde{Y}, \quad \tilde{\sigma}_{01}^{\mathrm{L}}(\tilde{Y}), \quad \tilde{\sigma}_{01}^{\mathrm{R}}(\tilde{Y}),
$$

respectively gives the highest weight vector of weight

$$
\left(Y+\Lambda_{l}, Y^{\dagger}+\Lambda_{r}\right), \quad\left(\circ_{0,1}^{(R)}\left(Y+\Lambda_{l}\right), Y^{\dagger}+\Lambda_{r}\right), \quad\left(Y+\Lambda_{l}, \stackrel{\circ}{0}_{0,1}^{(L)}\left(Y^{\dagger}+\Lambda_{r}\right)\right) .
$$

$\left(h=\frac{1}{2}\right): \quad I d e n t i f y \quad Y \in \mathscr{Y}_{l, r}$ with $\tilde{Y} \in \operatorname{Mat}(2 l+1,2 r+1)$ as

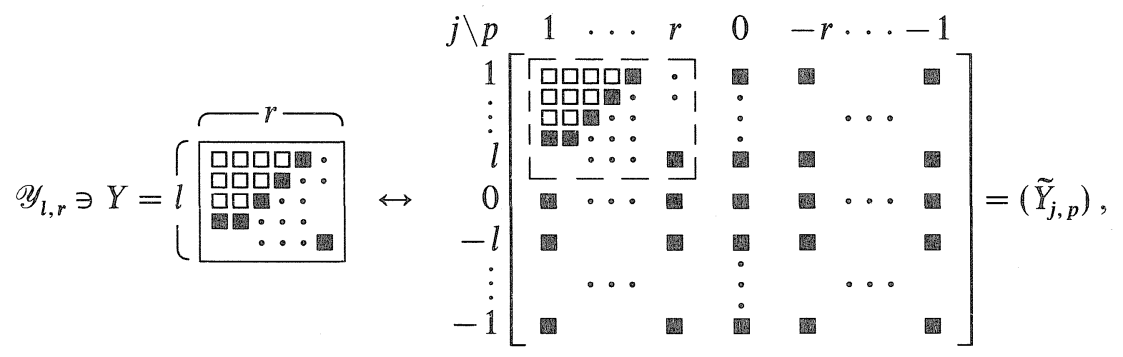

and put

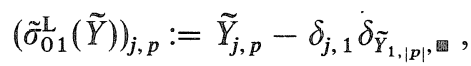

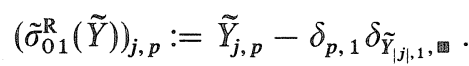

Then the vector $\left(\prod_{y_{j, p}=\square} \psi^{j, p}\left(-\frac{1}{2}\right)\right) \cdot 1$ for each of the matrices

$$
\left(y_{j, p}\right)=\tilde{Y}, \quad \tilde{\sigma}_{01}^{\mathrm{L}}(\tilde{Y}), \quad \tilde{\sigma}_{01}^{\mathrm{R}}(\tilde{Y}), \quad \tilde{\sigma}_{01}^{\mathrm{L}}\left(\tilde{\sigma}_{01}^{\mathrm{R}}(\tilde{Y})\right),
$$

respectively gives the highest weight vector of weight

$$
\left(Y,{ }^{t} Y\right), \quad\left(\sigma_{0,1}^{(R)}(Y),{ }^{t} Y\right), \quad\left(Y, \stackrel{\circ}{\sigma}_{0,1}^{(L)}\left({ }^{t} Y\right)\right), \quad\left(\dot{\sigma}_{0,1}^{(R)}(Y), \stackrel{\sigma}{0,1}^{(L)}\left({ }^{t} Y\right)\right)
$$

\section{List 4.2. $\hat{\mathrm{D}} \hat{\mathrm{B}}^{*}$}

$(h=0)^{* *}:$ Identify $Y \in \mathscr{Y}_{l, r}$ with $\tilde{Y} \in \operatorname{Mat}(2 l, 2 r+1)$ as

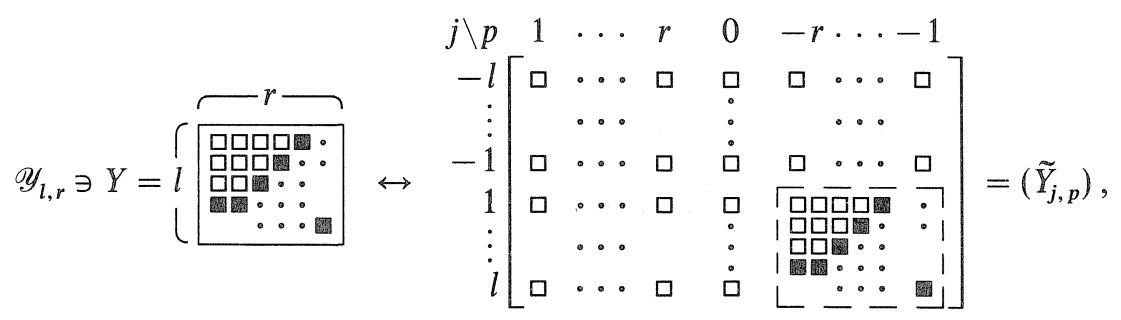

and define $\tilde{\sigma}_{01}^{\mathrm{L}}(\tilde{Y}), \tilde{\sigma}_{l-1, l}^{\mathrm{L}}(\tilde{Y})$ and $\tilde{\sigma}_{01}^{\mathrm{R}}(\tilde{Y}) \in \operatorname{Mat}(2 l, 2 r+1)$ by 


$$
\begin{aligned}
\left(\tilde{\sigma}_{01}^{\mathrm{L}}(\tilde{Y})\right)_{j, p} & :=\tilde{Y}_{j, p}-\operatorname{sgn}(j) \delta_{|j|, 1} \delta_{\tilde{Y}_{1,-1}, \text { घ }} \delta_{\tilde{Y}_{-1,-1}, \square} \delta_{\tilde{Y}_{1, p}, \text { 冈ा }}, \\
\left(\tilde{\sigma}_{l-1, l}^{\mathrm{L}}(\tilde{Y})\right)_{j, p} & :=\tilde{Y}_{j, p}+\delta_{j, l} \delta_{\tilde{Y}_{l,-|p|}, \square}, \\
\left(\tilde{\sigma}_{01}^{\mathrm{R}}(\tilde{Y})\right)_{j, p} & :=\tilde{Y}_{j, p}+\delta_{p,-1} \delta_{\tilde{Y}_{1,-1}, \square} \delta_{\tilde{Y}_{-1,-1}, \square} \delta_{\tilde{Y}_{|j|,-1}, \square},
\end{aligned}
$$

respectively. Then the vector**

$$
\left(\prod_{y_{j, p}=\square} \psi_{j, p}\left(\mu_{0}\right)\right) \cdot 1
$$

for

$$
\begin{array}{rll}
\left(y_{j, p}\right)=\tilde{Y}, \quad \tilde{\sigma}_{01}^{\mathrm{L}}(\tilde{Y}), & \tilde{\sigma}_{01}^{\mathrm{R}}(\tilde{Y}), \\
\tilde{\sigma}_{l-1, l}^{\mathrm{L}}(\tilde{Y}), \quad \tilde{\sigma}_{l-1, l}^{\mathrm{L}}\left(\tilde{\sigma}_{01}^{\mathrm{L}}(\tilde{Y})\right), & \tilde{\sigma}_{l-1, l}^{\mathrm{L}}\left(\tilde{\sigma}_{01}^{\mathrm{R}}(\tilde{Y})\right)
\end{array}
$$

respectively gives the highest weight vector of weight

$$
\begin{array}{ccc}
\left(Y+\Lambda_{l}, Y^{\dagger}\right), & \left(\dot{\sigma}_{0,1}^{(R)}\left(Y+\Lambda_{l}\right), Y^{\dagger}\right), & \left(Y+\Lambda_{l}, \circ_{0,1}^{(L)}\left(Y^{\dagger}\right)\right), \\
\left(\circ_{l-1, l}^{(R)}\left(Y+\Lambda_{l}\right), Y^{\dagger}\right), & \left(\dot{\circ}_{l-1, l}^{(R)}\left(\circ_{01}^{(R)}\left(Y+\Lambda_{l}\right)\right), Y^{\dagger}\right), & \left(\dot{\sigma}_{l-1, l}^{(R)}\left(Y+\Lambda_{l}\right), \dot{\sigma}_{01}^{(L)}\left(Y^{\dagger}\right)\right) .
\end{array}
$$$$
\left(\boldsymbol{h}=\frac{1}{2}\right): \quad \text { Identify } Y \in \mathscr{Y}_{l, r} \text { with } \tilde{Y} \in \operatorname{Mat}(2 l, 2 r+1) \text { as }
$$

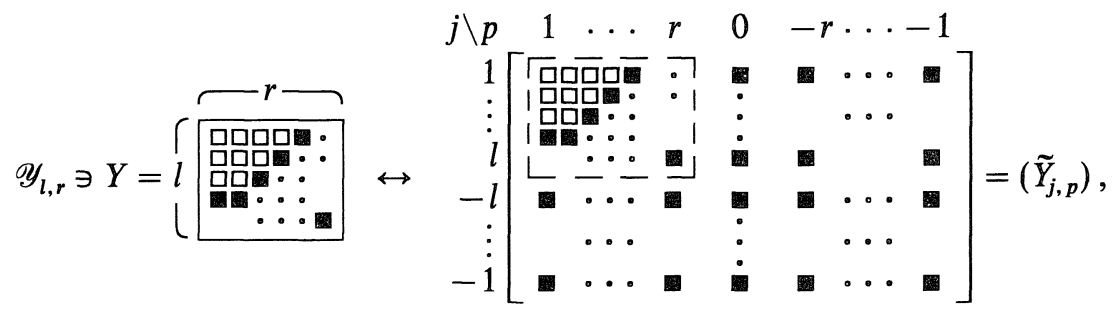

and define

$$
\begin{aligned}
& \left(\tilde{\sigma}_{01}^{\mathrm{L}}(\tilde{Y})\right)_{j, p}:=\tilde{Y}_{j, p}-\delta_{j, 1} \delta_{\tilde{Y}_{1,|p|}, \mathbf{m}} \\
& \left(\tilde{\sigma}_{l-1, l}^{\mathrm{L}}(\tilde{Y})\right)_{j, p}:=\tilde{Y}_{j, p}+\operatorname{sgn}(j) \delta_{|j|, l} \delta_{\tilde{Y}_{l, 1}, \square} \delta_{\tilde{Y}_{-l, 1}, \mathbf{\Xi}} \delta_{\tilde{Y}_{l, p}, \square}, \\
& \left(\tilde{\sigma}_{01}^{\mathrm{R}}(\tilde{Y})\right)_{j, p}:=\tilde{Y}_{j, p}-\delta_{p, 1} \delta_{\tilde{Y}_{l, 1}, \mathbf{m}} \delta_{\tilde{Y}_{-l, 1}, \mathbf{m}} \delta_{\tilde{Y}_{|j|, 1}, \mathbf{m}}
\end{aligned}
$$

Then the vector

$$
\left(\vec{\prod}_{y_{j, p}=a} \psi^{j, p}\left(-\frac{1}{2}\right)\right) \cdot 1
$$

for

$$
\begin{array}{ccc}
\left(y_{j, p}\right)=\tilde{Y}, \quad \tilde{\sigma}_{l-1, l}^{\mathrm{L}}(\tilde{Y}), & \tilde{\sigma}_{01}^{\mathrm{R}}(\tilde{Y}), \\
\tilde{\sigma}_{01}^{\mathrm{L}}(\tilde{Y}), & \tilde{\sigma}_{l-1, l}^{\mathrm{L}}\left(\tilde{\sigma}_{01}^{\mathrm{L}}(\tilde{Y})\right), & \tilde{\sigma}_{01}^{\mathrm{L}}\left(\tilde{\sigma}_{01}^{\mathrm{R}}(\tilde{Y})\right),
\end{array}
$$

respectively gives the highest weight vector of weight

$$
\begin{aligned}
& \left(Y,{ }^{t} Y\right), \quad\left(\dot{\sigma}_{l-1, l}^{(R)}(Y),{ }^{t} Y\right), \quad\left(Y, \dot{\sigma}_{0,1}^{(L)}\left({ }^{t} Y\right)\right), \\
& \left(\sigma_{0,1}^{(R)}(Y),{ }^{t} Y\right), \quad\left(\sigma_{l-1, l}^{(R)}\left(\sigma_{0,1}^{(R)}(Y)\right),{ }^{t} Y\right), \quad\left({ }^{\circ}(R),(Y),{ }^{\circ}(L),\left({ }^{t} Y\right)\right) .
\end{aligned}
$$


List 4.2. $\hat{\mathrm{D}} \hat{\mathrm{D}}$ *

$(\boldsymbol{h}=\mathbf{0})^{* *}: \quad$ Identify $Y \in \mathscr{Y}_{l, r}$ with $\tilde{Y} \in \operatorname{Mat}(2 l, 2 r)$ as

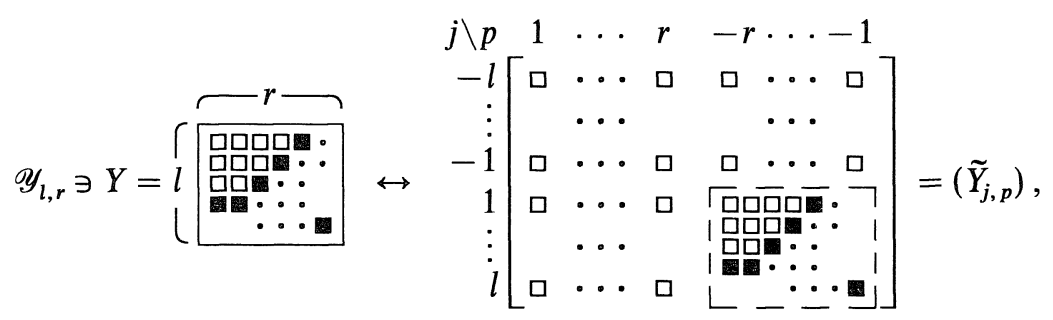

and define

$$
\begin{aligned}
\left(\tilde{\sigma}_{01}^{\mathrm{L}}(\tilde{Y})\right)_{j, p} & :=\tilde{Y}_{j, p}-\operatorname{sgn}(j) \delta_{|j|, 1} \delta_{\tilde{Y}_{1,-1}, \bowtie} \delta_{\tilde{Y}_{-1,-1}, \square} \delta_{\tilde{Y}_{1, p}, \varpi}, \\
\left(\tilde{\sigma}_{l-1, l}^{\mathrm{L}}(\tilde{Y})\right)_{j, p} & :=\tilde{Y}_{j, p}+\delta_{j, l} \delta_{\tilde{Y}_{l,-r}, \square} \delta_{\tilde{Y}_{l, r}, \square} \delta_{\tilde{Y}_{l,-|p|}, \square}, \\
\left(\tilde{\sigma}_{01}^{\mathrm{R}}(\tilde{Y})\right)_{j, p} & :=\tilde{Y}_{j, p}+\delta_{p,-1} \delta_{\tilde{Y}_{1,-1}, \square} \delta_{\tilde{Y}_{-1,-1}, \square} \delta_{\tilde{Y}_{|j|,-1}, \square}, \\
\left(\tilde{\sigma}_{r-1, r}^{\mathrm{R}}(\tilde{Y})\right)_{j, p} & :=\tilde{Y}_{j, p}+\operatorname{sgn}(p) \delta_{|p|, r} \delta_{\tilde{Y}_{l, r}, \square} \delta_{\tilde{Y}_{l,-r}, \boxplus} \delta_{\tilde{Y}_{j,-r}, \varpi}
\end{aligned}
$$

Then the vector**

$$
\left(\vec{\prod}_{y_{j, p}=\mathbf{m}} \psi_{j, p}\left(\mu_{0}\right)\right) \cdot 1
$$

for

$$
\begin{array}{rcc}
\left(y_{j, p}\right)=\tilde{Y}, \quad \tilde{\sigma}_{01}^{\mathrm{L}}(\tilde{Y}), & \tilde{\sigma}_{01}^{\mathrm{R}}(\tilde{Y}), \\
\tilde{\sigma}_{l-1, l}^{\mathrm{L}}(\tilde{Y}), & \tilde{\sigma}_{l-1, l}^{\mathrm{L}}\left(\tilde{\sigma}_{01}^{\mathrm{L}}(\tilde{Y})\right), & \tilde{\sigma}_{l-1, l}^{\mathrm{L}}\left(\tilde{\sigma}_{01}^{\mathrm{R}}(\tilde{Y})\right) . \\
\left.\tilde{\sigma}_{r-1, r}^{\mathrm{R}}(\tilde{Y})\right), & \tilde{\sigma}_{r-1, r}^{\mathrm{R}}\left(\tilde{\sigma}_{01}^{\mathrm{L}}(\tilde{Y})\right), & \tilde{\sigma}_{r-1, r}^{\mathrm{R}}\left(\tilde{\sigma}_{01}^{\mathrm{R}}(\tilde{Y})\right),
\end{array}
$$

respectively gives the highest weight vector of weight

$$
\begin{aligned}
& \left(Y, Y^{\dagger}\right), \quad\left(\stackrel{\circ}{\sigma}(R), 1_{0}(Y), Y^{\dagger}\right), \quad\left(Y, \stackrel{\circ}{\sigma}_{0,1}^{(L)}\left(Y^{\dagger}\right)\right), \\
& \left(\dot{\sigma}_{l-1, l}^{(R)}(Y), Y^{\dagger}\right), \quad\left(\dot{\sigma}_{l-1, l}^{(R)}\left(\dot{\sigma}_{0,1}^{(R)}(Y)\right), Y^{\dagger}\right), \quad\left(\dot{\sigma}_{l-1, l}^{(R)}(Y), \stackrel{\circ}{\sigma}(L), 1^{(L)}\left(Y^{\dagger}\right)\right) . \\
& \left(\dot{\sigma}_{r-1, r}^{(R)}(Y), Y^{\dagger}\right), \quad\left(\dot{\sigma}_{r-1, r}^{(R)}\left(\sigma_{0,1}^{\circ(R)}(Y)\right), Y^{\dagger}\right), \quad\left(\dot{\sigma}_{r-1, r}^{(R)}(Y), \dot{\sigma}_{0,1}^{(L)}\left(Y^{\dagger}\right)\right) .
\end{aligned}
$$

$\left(\boldsymbol{h}=\frac{1}{2}\right): \quad$ Identify $Y \in \mathscr{Y}_{l, r}$ with $\tilde{Y} \in \operatorname{Mat}(2 l, 2 r)$ as

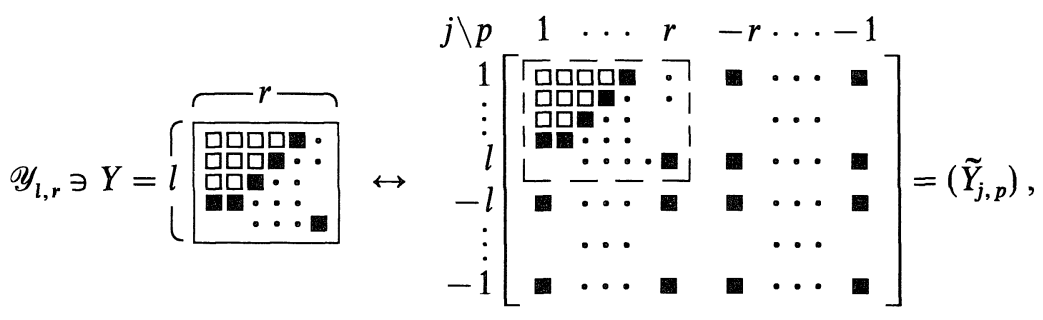

and define 


$$
\begin{aligned}
\left(\tilde{\sigma}_{01}^{\mathrm{L}}(\tilde{Y})\right)_{j, p} & :=\tilde{Y}_{j, p}-\delta_{j, 1} \delta_{\tilde{Y}_{1, r}, \square} \delta_{\tilde{Y}_{1,-r}, \square} \delta_{\tilde{Y}_{1,|p|}, \square}, \\
\left(\tilde{\sigma}_{l-1, l}^{\mathrm{L}}(\tilde{Y})\right)_{j, p} & :=\tilde{Y}_{j, p}+\operatorname{sgn}(j) \delta_{|j|, l} \delta_{\tilde{Y}_{l, 1}, \square} \delta_{\tilde{Y}_{-l, 1}, \square} \delta_{\tilde{Y}_{l, p}, \square}, \\
\left(\tilde{\sigma}_{01}^{\mathrm{R}}(\tilde{Y})\right)_{j, p} & :=\tilde{Y}_{j, p}-\delta_{p, 1} \delta_{\tilde{Y}_{l, 1}, \square} \delta_{\tilde{Y}_{-l, 1}, \square} \delta_{\tilde{Y}_{\mid l, 1}, \square}, \\
\left(\tilde{\sigma}_{r-1, r}^{\mathrm{R}}(\tilde{Y})\right)_{j, p} & :=\tilde{Y}_{j, p}+\operatorname{sgn}(p) \delta_{|p|, r} \delta_{\tilde{Y}_{l, r}, \square} \delta_{\tilde{Y}_{1,-r}, \square} \delta_{\tilde{Y}_{j, r}, \square} \cdot
\end{aligned}
$$

Then the vector

$$
\left(\overrightarrow{\mathbb{I}}_{y_{j, p}=\square} \psi^{j, p}\left(-\frac{1}{2}\right)\right) \cdot 1
$$

for

$$
\begin{array}{rcc}
\left(y_{j, p}\right)=\tilde{Y}, \quad \tilde{\sigma}_{01}^{\mathrm{L}}(\tilde{Y}), & \tilde{\sigma}_{r-1, r}^{\mathrm{R}}(\tilde{Y}), \\
\tilde{\sigma}_{01}^{\mathrm{R}}(\tilde{Y}), & \tilde{\sigma}_{01}^{\mathrm{L}}\left(\tilde{\sigma}_{01}^{\mathrm{R}}(\tilde{Y})\right), & \tilde{\sigma}_{01}^{\mathrm{R}}\left(\tilde{\sigma}_{r-1, r}^{\mathrm{R}}(\tilde{Y})\right), \\
\left.\tilde{\sigma}_{l-1, l}^{\mathrm{L}}(\tilde{Y})\right), & \tilde{\sigma}_{l-1, l}^{\mathrm{L}}\left(\tilde{\sigma}_{01}^{\mathrm{L}}(\tilde{Y})\right), & \tilde{\sigma}_{l-1, l}^{\mathrm{L}}\left(\tilde{\sigma}_{r-1, r}^{\mathrm{R}}(\tilde{Y})\right),
\end{array}
$$

respectively gives the highest weight vector of weight

$$
\begin{aligned}
& \left(Y,{ }^{t} Y\right), \quad\left(\sigma_{0,1}^{\circ(R)}(Y),{ }^{t} Y\right), \quad\left(\mathbb{Y}, \stackrel{\sigma}{\sigma}(L) 1, r^{(L)}\left({ }^{t} Y\right)\right),
\end{aligned}
$$

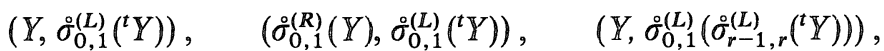

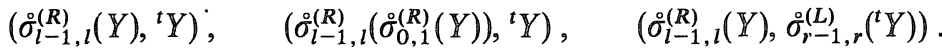

Notations for List 4 .2. $\square$ As in List 3.2 we use the notation $\vec{\Pi}$, and identify $\square=0$, $₫=1$ so that $\square+1=$ 困, etc. For example, read List $4.2 . \hat{\mathbb{D}} \hat{\mathrm{B}}, h=1 / 2$ as follows: for the diagram

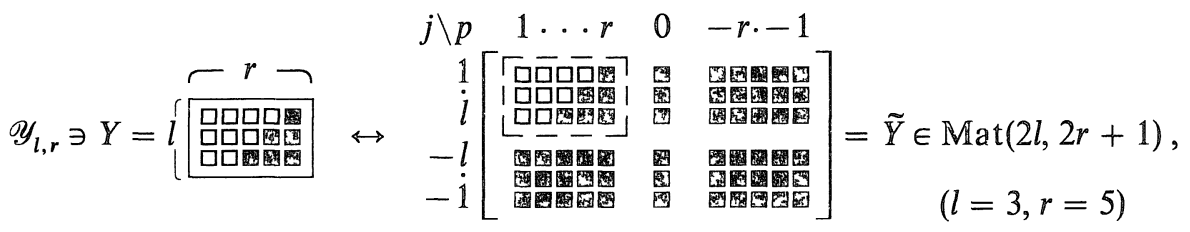

$\tilde{\sigma}_{01}^{\mathrm{L}}(\tilde{Y}), \tilde{\sigma}_{l-1, l}^{\mathrm{L}}(\tilde{Y}), \tilde{\sigma}_{01}^{\mathrm{R}}(\tilde{Y}) \in \operatorname{Mat}(2 l, 2 r+1)$ are respectively given by
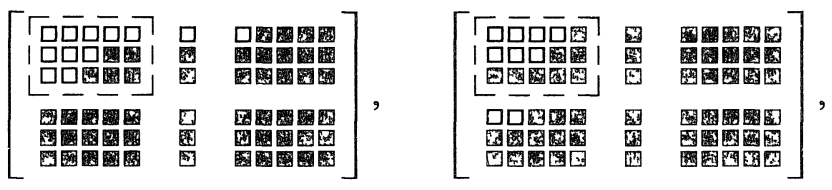

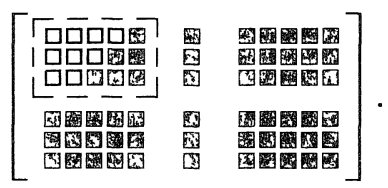

(unchanged)

$\square$ The weight (rather, its classical part) of each vector in the list is given in the manner: (weight for $\mathfrak{g}_{\mathfrak{L}}^{\wedge}$, weight for $\left.\mathfrak{g}_{\mathbb{R}}\right)$.

0 We also use:

(*) In these cases, for a diagram automorphism $\sigma \in \mathbb{A u t}_{\mathbb{C}} \tilde{\mathfrak{h}}^{*}(\S \S 1.5 .2 \sim 5)$ and $l \in \mathbb{Z}_{>0}$ we define $\sigma^{(l)}: \mathfrak{h}^{*} \rightarrow \mathfrak{h}^{*}$ by

$$
\sigma\left(\lambda+l \hat{\Lambda}_{0}\right) \equiv \dot{\sigma}^{(l)}(\lambda)+l \hat{\Lambda}_{0} \quad \bmod \mathbb{C} \delta \quad \text { for } \lambda \in \mathfrak{h}^{*} .
$$


(**) In these cases $(h=0)$, we define $\psi_{j, p}\left(\mu_{0}\right)$ as follows.

$$
\psi_{j, p}\left(\mu_{0}\right):= \begin{cases}\psi_{j, p}(0) & : \text { if } \psi_{j, p}(0) \in W_{N}^{\mathbb{Z},-} \\ \psi_{j, p}(-1) & : \text { otherwise }\end{cases}
$$

Remark 4.2(i). In contrast with the finite dimensional case, there are some irreducible integrable highest weight modules that does not appear in our module $\bigwedge\left(W_{N}^{\mathbb{Z}+h \cdot-}\right): \mathbb{L}^{\mathrm{o}(2 r)^{\wedge}}\left(\hat{\Lambda}_{0}+\hat{\Lambda}_{r}\right)$ for example. We have to consider then the tensor module $\bigwedge\left(W_{N}^{\mathbb{Z},-}\right) \otimes \wedge\left(W_{N^{\prime}}^{\mathbb{Z}+1 / 2,-}\right)$ to obtain all such representations of $\mathrm{D}(n)^{\wedge}$.

On the other hand, for $\mathfrak{s l}(r)^{\wedge}$ and $\mathfrak{s p}(2 r)^{\wedge}$ it is remarkable that all level $l$ irreducible highest weight modules appear in $\bigwedge\left(W_{2 l r}^{\mathbb{Z}+h,-}\right)$ and $\bigwedge\left(W_{4 l r}^{\mathbb{Z}+h,-}\right)$ respectively.

(ii) By the well-known linear independence of characters [Kac, Proposition 13.9], Theorem 4.2 is equivalent to the character identity

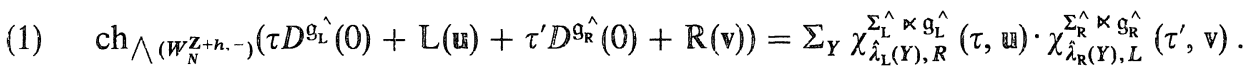

(iii) The explicit form of decomposition (0) in Theorem 4.2 for (Case $\hat{A} \hat{A}, h=0$ ) appears in [JM, Proposition 2.5] as the character identity (1). For (Case $\widehat{C} \widehat{C}, h=0$ ), (0) is given in [KP1], to which we partly owe the simplification of the proof of Theorem 4.2 in this paper.

国

Example $4 . z_{0}$ Here we will explain the highest weight vectors for Case $\hat{A} \hat{A}, h=1 / 2$ in a graphical fashion. As in Example 3.2 we consider the case of $l=3, r=2$.

Let us first introduce the set $\mathfrak{M}=\mathfrak{M}_{l, r}^{\mathbb{Z}+1 / 2}$ of Maya diagrams of $(l \times r)$-components and the assignation $\mathfrak{M} \ni M \mapsto|M\rangle \in \bigwedge\left(W_{2 l r}^{\mathbb{Z}+1 / 2,-}\right)$ from $\mathfrak{M}$ to pure spinors in the Fermion Fock space as follows: we put

$$
\mathfrak{M}:=\left\{\left(m_{j, p}(v)\right)_{\substack{v \in \mathbb{Z}+1 / 2 \\
1 \leq j \leq l, 1 \leq p \leq r}} \mid \begin{array}{ll}
m_{j, p}(v)=\square & \text { or } \quad \text { 因, } \\
m_{j, p}(v)=\square & (v \ll 0), \quad m_{j, p}(v)=\text { 图 } \quad(v \gg 0)
\end{array}\right\}
$$

and

(2) $\quad \mathfrak{M} \ni M=\left(m_{j, p}(v)\right) \mapsto|M\rangle:=\left(\prod_{\substack{v<0, j, p \\ m_{j, p}(v)=\Xi}}^{\overrightarrow{ }} \psi_{j, p}(v)\right)\left(\prod_{\substack{v>0, j, p \\ m_{\jmath, p}(v)=\square}}^{\rightarrow} \psi^{j, p}(-v)\right) \cdot 1 \in \bigwedge\left(W_{2 l r}^{\mathbf{Z}+1 / 2,-}\right)$.

The set $\{|M\rangle \mid M \in \mathfrak{M}\}$ forms a basis of $\bigwedge\left(W_{2 l r}^{\mathbb{Z}+1 / 2,-}\right)$. For example,

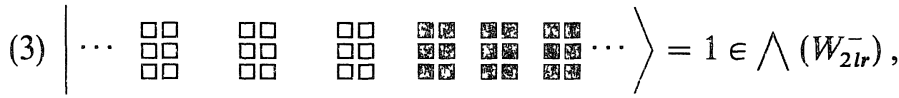

$\cdots-5 / 2-3 / 2-1 / 2 \quad 1 / 2 \quad 3 / 2 \quad 5 / 2 \cdots \rightarrow v$

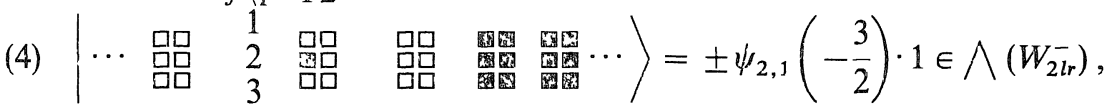

$j \backslash p \quad 12$ $\cdots-5 / 2 \quad-3 / 2-1 / 21 / 23 / 2 \cdots$

(5)

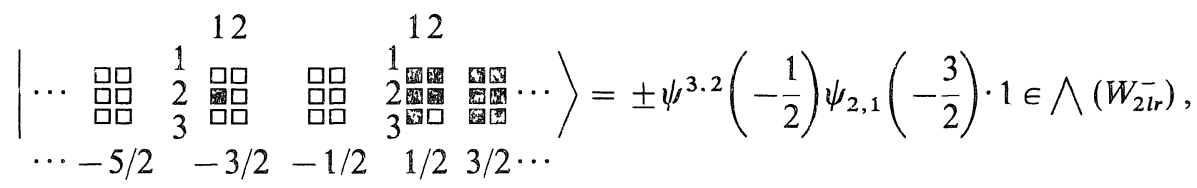


and so on. Hereafter \pm means that we will forget the information of the ordering of fermion operators $\psi_{p, j}(v)$ in the expression.

Now we can express the highest weight vectors graphically. We consider the highest weight vector $\left|\left(y_{j, p}\right)\right\rangle:=\left|\sum_{j, p} y_{j, p} \varepsilon_{j, p}\right\rangle \in \bigwedge\left(W_{12}^{\mathbb{Z}+1 / 2,-}\right)(12=2 l r)$ given in List $\hat{\mathbb{A}} \hat{A}$ that corresponds to the data

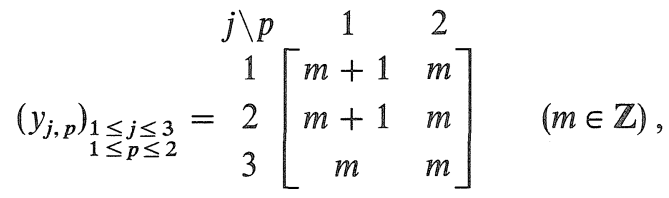

for example. Forgetting the sign \pm , the definition $(8.1(5))$ of $\left|\left(y_{j, p}\right)\right\rangle$ reads as follows:

for $m \geq 0$,

$$
\left|\left[\begin{array}{cc}
m+1 & m \\
m+1 & m \\
m & m
\end{array}\right]\right\rangle:= \pm \psi^{1,1}\left(-m-\frac{1}{2}\right) \cdot \psi^{2,1}\left(-m-\frac{1}{2}\right) \cdot \overrightarrow{\prod_{j, p}} \underset{\mu=1}{\stackrel{m}{\rightarrow}} \psi^{j, p}\left(-\mu+\frac{1}{2}\right) \cdot 1
$$

and for $m<0$,

$$
\begin{aligned}
\left.\llbracket\left[\begin{array}{cc}
m+1 & m \\
m+1 & m \\
m & m
\end{array}\right]\right\rangle:= & \pm \psi_{1,2}\left(m+\frac{1}{2}\right) \psi_{2,2}\left(m+\frac{1}{2}\right) \psi_{3,1}\left(m+\frac{1}{2}\right) \psi_{3,2}\left(m+\frac{1}{2}\right) \\
& \times \underset{j, p}{\rightarrow} \stackrel{-m-1}{\rightarrow} \psi_{\mu=1}^{\rightarrow}\left(-\mu+\frac{1}{2}\right) \cdot 1 .
\end{aligned}
$$

Formulae (7) and (8) are easily interpreted in terms of Maya diagrams, and we have the following expression (9).

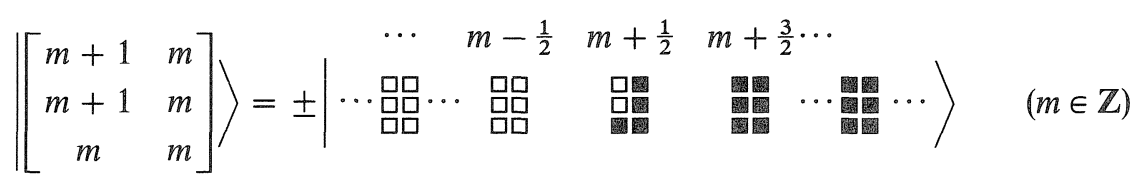

With the help of Maya diagrams, the action of the auxiliary automorphism $\tilde{\sigma}_{c y c}^{\#} \in \operatorname{Aut}\left(\bigwedge\left(W_{2 l r}^{\mathbb{Z}+1 / 2,-}\right)\right)$ to the highest weight vector can be also described as follows. (See $\S 8.2$ for the definition of $\tilde{\sigma}_{c y c}^{\#}$. Similarly to $1.3 .4(9)$ it is defined as a composition of some "translation" $T_{\gamma}^{\sim}$ and "index changing" $(1, \ldots, l)^{\sim}$ associated to the cycle $(1, \ldots, l) \in \mathfrak{S}_{l}$.)

$$
\begin{aligned}
& \tilde{\sigma}_{c y c}^{\#}\left|\left[\begin{array}{cc}
m+1 & m \\
m+1 & m \\
m & m
\end{array}\right]\right\rangle=T_{\left(\sum_{p=1}^{r} \varepsilon_{1, p}\right)}^{\sim} \circ(1, \ldots, l)^{\sim}\left|\left[\begin{array}{cc}
m+1 & m \\
m+1 & m \\
m & m
\end{array}\right]\right\rangle \\
& \text { ‥ } m-\frac{1}{2} m+
\end{aligned}
$$

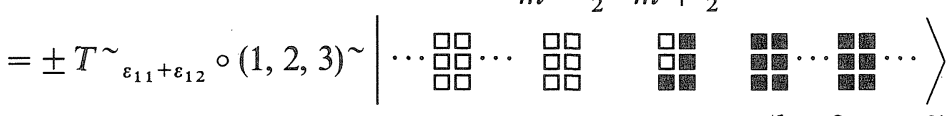

$$
\begin{aligned}
& (l=3, r=2)
\end{aligned}
$$




$$
\begin{aligned}
& \text { ․ } m-\frac{1}{2} \quad m+\frac{1}{2}
\end{aligned}
$$

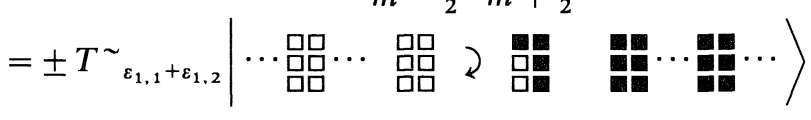

$$
\begin{aligned}
& \text { ․ } m-\frac{1}{2} \quad \downarrow \downarrow m+\frac{3}{2} \quad \cdots
\end{aligned}
$$

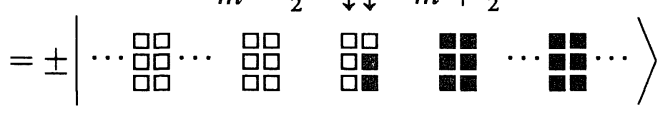

$$
\begin{aligned}
& \left.= \pm\left[\begin{array}{cc}
m+1 & m+1 \\
m+1 & m \\
m+1 & m
\end{array}\right]\right\rangle \text {. }
\end{aligned}
$$

For other cases ( $\hat{\mathrm{C}} \hat{\mathrm{C}}$, ÔÔ) of Theorem 4.2, we can similarly express the highest weight vectors by Maya diagrams. The graphical data $\left(Y_{j, p}^{\sim}\right)$ of highest weight vectors given in List 4.2 are then interpreted into the $(-h)$-mode part $\left(m_{j, p}(-h)\right)(h=0$ or $1 / 2)$ of their Maya diagram expressions

$$
\left|\left(m_{j, p}(v)\right)_{j, p ; v \in \mathbf{Z}+h}\right\rangle .
$$

\subsection{Proof of Theorem 4.2; in cases of $h=0$}

4.3.1. Let $\mathfrak{g}=\mathfrak{g}_{0} \oplus \mathfrak{g}_{1}$ be a simple Lie algebra and its Cartan decomposition in Section 3.3. Putting

$$
\mathfrak{g}_{0}^{\wedge}:=\mathfrak{g}_{0} \otimes \mathbf{C}\left[t, t^{-1}\right] \oplus \mathbf{C} c \quad \text { and } \quad \mathfrak{g}_{1}^{\wedge}:=\mathfrak{g}_{1} \otimes \mathbb{C}\left[t, t^{-1}\right] \text {, }
$$

the affinization $\mathfrak{g}^{\wedge}$ also has a $\mathbf{Z}_{2}$-gradation $\mathfrak{g}^{\wedge}=\mathfrak{g}_{0}^{\wedge} \oplus \mathfrak{g}_{1}^{\wedge}$ and $\mathfrak{g}_{0}^{\wedge}$ acts on $\mathfrak{g}_{1}^{\wedge}$ with preserving the standard form $(\mid)([\mathrm{Kac}, \S 6.2])$. Choosing an isotropic decomposition $\mathfrak{g}_{1}^{\wedge}=\mathfrak{g}_{1}^{\wedge,+} \oplus \mathfrak{g}_{1}^{\wedge,-}$ given by

$$
\mathfrak{g}_{1}^{\wedge}, \pm:=\mathfrak{g}_{1}^{\wedge} \cap \mathfrak{n}_{ \pm}^{\sim},
$$

we consider the Clifford algebra $\mathscr{C}\left(\mathfrak{g}_{\hat{1}},(\mid)\right)$ and its spin representation on $\bigwedge\left(\mathfrak{g}_{1}^{\wedge},-\right)$. Then $\mathfrak{v}\left(\mathfrak{g}_{1}\right)^{\wedge}$ acts on $\bigwedge\left(\mathfrak{g}_{1}^{\wedge},-\right)$ as in Section 2. On the other hand there is a Lie algebra monomorphism

$$
I^{\wedge}: \mathfrak{g}_{0}^{\wedge} \rightarrow \mathfrak{v}\left(\mathfrak{g}_{1}\right)^{\wedge} \quad \text { induced by } I^{\wedge}(x(k)):=I(x)(k),
$$

where $I: g_{0} \rightarrow \mathfrak{o}\left(g_{1}\right)$ is the inclusion map (3.3.1(1)). Composing these maps we obtain the representation of $\mathfrak{g}_{0}^{\wedge}$ on $\bigwedge\left(\mathfrak{g}_{1}^{\wedge,-}\right)$.

Lemma 4.3 [KP1, Proposition 1]. As a $\mathfrak{g}_{0}^{\wedge}$-module,

$$
\bigwedge^{\text {even }}\left(\mathfrak{g}_{1}^{\wedge}\right) \simeq \bigoplus_{w \in W_{1}^{\wedge}, \operatorname{sgn} w=1} \mathbf{L}^{\hat{g_{0}}\left(w(\hat{\rho})-\hat{\rho}_{0}\right)}
$$

and

$$
\bigwedge^{\text {odd }}\left(\mathfrak{g}_{1}^{\wedge}\right) \simeq \bigoplus_{w \in W_{1}^{\wedge}, \operatorname{sgn} w=-1} \mathbf{L}^{\mathfrak{g}_{0}}\left(w(\hat{\rho})-\hat{\rho}_{0}\right),
$$

where $W_{1}^{\wedge}:=\left\{w \in W^{\wedge} \mid w\left(\hat{\Delta}_{0}^{+}\right) \subset \hat{\Delta}^{+}\right\}, W^{\wedge}$ denotes the Weyl group of $\mathfrak{g}^{\wedge}$, and $\rho$ (resp. $\left.\rho_{0}\right)$ the sum of fundamental weights of $\mathfrak{g}^{\wedge}$ (resp. $\left.\mathfrak{g}_{0}^{\wedge}\right)$. 
Proof. We can prove this similarly to Lemma 3.3, if we suitably modify the notions there.

Proof of Theorem 4.2, Case $\widehat{\mathbb{A}} \hat{\mathbb{A}}, \widehat{\mathbb{C}} \widehat{\mathbb{C}}, \widehat{\mathbb{D}} \hat{\mathbb{B}}$ and $\widehat{\mathbb{D}} \hat{\mathbb{D}}$ for $h=0$. In these cases we can derive the theorem similarly to Theorem 3.2 for the Case $A A, C C, D B$ and $D D$, using Lemma 4.3 above. The fact that List 4.2 actually gives highest weight vectors is straightforwardly verified.

4.3.2. As for the remaining case of Theorem 4.2 we prove them by case-by-case verification of corresponding character identities. For that purpose we will utilize the "complementary decomposition formula" obtained by Jimbo-Miwa [JM, Proposition 2.4 and Table $\mathbb{I I}]$.

Proof of Theorem 4.2 for Case $\widehat{B} \hat{B}, h=0$. We have

(1) $\operatorname{ch}_{(2 l+1)(2 r+1)}^{\mathbb{Z}}\left(\tau D^{\text {Clif }}(0)+\mathbb{L}(\right.$ \& $\left.)+\mathbb{R}(\mathrm{v})\right) \quad$ (see $2.4(15)$ for $D^{\text {Clif }}$ and 4.1 for $\mathbb{L}($ an $), \mathbb{R}(\mathrm{v})$ )

$$
\begin{aligned}
& =\frac{\eta(2 \tau)}{\eta(\tau)} \prod_{j=1}^{l} \frac{-i \theta\left(\tau, u_{j}+\frac{1}{2}\right)}{\eta(\tau)} \prod_{p=1}^{r} \frac{-i \theta\left(\tau, v_{p}+\frac{1}{2}\right)}{\eta(\tau)} \\
& \times \prod_{j=1}^{l} \prod_{p=1}^{r} \frac{-\theta\left(\tau, u_{j}+v_{p}+\frac{1}{2}\right) \theta\left(\tau, u_{j}-v_{p}+\frac{1}{2}\right)}{\eta(\tau)^{2}} \\
& =\prod_{p=1}^{r} \frac{\theta\left(\tau, v_{p}+\frac{1}{2}\right)}{\theta\left(\tau, v_{p}+1\right)} \times q^{(2 l+2 r+1) / 24} \operatorname{ch}_{2 l+2 r+1}^{\mathbb{Z}}\left(\tau D_{0}+\left(\mathbb{\nabla} \oplus\left(\mathrm{v}+\frac{\mathbb{1}}{2}\right)\right)\right) \\
& \times \mathscr{D}^{\mathfrak{o}(2 l+2 r+1)^{\wedge}}\left(\tau, \mathbb{u} \oplus\left(\mathrm{v}+\frac{\mathbb{1}}{2}\right)\right) / \mathscr{D}^{\mathfrak{o}(2 l+1)^{\wedge}}(\tau, \mathbb{\mathbb { d }}) \mathscr{D}^{\mathfrak{o}(2 r+1)^{\wedge}}\left(\tau, \mathrm{v}+\frac{\mathbb{1}}{2}\right) \\
& =\prod_{p=1}^{r} \frac{\theta\left(\tau, v_{p}+\frac{1}{2}\right)}{\theta\left(\tau, v_{p}+1\right)} \\
& \times \Sigma_{Y \in \mathscr{V}_{l, r}}(-1)^{\left[Y^{\dagger}\right]} \chi_{Y+\Lambda_{l}, 2 r+1}^{\left\langle\sigma_{01}\right\rangle \times \mathfrak{o}(2 l+1)^{\wedge}}(\tau, \mathbb{R}) \cdot \chi_{Y^{+}+\Lambda_{r}, 2 l+1}^{\left\langle\sigma_{01}\right\rangle \times \mathfrak{o}(2 r+1)^{\wedge}}\left(\tau, \mathbb{v}+\frac{\mathbb{1}}{2}\right)
\end{aligned}
$$

(Complementary decomposition formula $\left[\mathbb{J M}\right.$, Table $\left.\mathbb{I I},(6) 7^{\circ}\right]$ for $\tilde{\Lambda}=\hat{\Lambda}_{l+r}=\left(\Lambda_{l+r}, 1\right)$ )

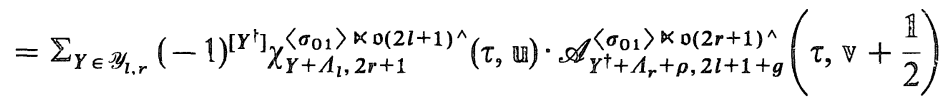

$$
\begin{aligned}
& \times \mathbb{\prod}_{p=1}^{r} \frac{\theta\left(\tau, v_{p}+\frac{1}{2}\right)}{-\theta\left(\tau, v_{p}\right)} / \mathscr{D}^{\mathfrak{o}(2 r)^{\wedge}}\left(\tau, \mathbb{v}+\frac{\mathbb{1}}{2}\right) \mathbb{M}_{p=1}^{r} \theta\left(\tau, v_{p}+\frac{1}{2}\right) \\
& =\Sigma_{Y \in \mathscr{Y}_{l, r}}(-1)^{2\left(\left[Y^{4}\right]+r+r(r-1) / 2\right)} \\
& \times \chi_{Y+\Lambda_{l}, 2 r+1}^{\left\langle\sigma_{01}\right\rangle \times \mathrm{o}(2 l+1)^{\wedge}}(\tau, \mathbb{\nabla}) \mathscr{A}_{Y^{+}+\Lambda_{r}+\rho, 2 l+1+g}^{\left\langle\sigma_{01}\right\rangle \ltimes \mathrm{o}(2 r+1) \wedge}(\tau, \mathrm{v}) / \mathscr{D}^{\mathfrak{o}(2 r)^{\wedge}}(\tau, \mathrm{v}) \prod_{p} \theta\left(\tau, v_{p}\right) \\
& =\Sigma_{Y \in \mathscr{Y}_{l, r}} \chi_{Y+\Lambda_{l}, 2 r+1}^{\left\langle\sigma_{01}\right\rangle \propto \mathrm{O}(2 l+1)^{\wedge}}(\tau, \text { 过 }) \cdot \chi_{Y^{+}+\Lambda_{r}, 2 l+1}^{\left\langle\sigma_{01}\right\rangle \ltimes \mathrm{O}(2 r+1)^{\wedge}}(\tau, \mathbb{\mathrm { v }})
\end{aligned}
$$

for $\mathbb{a}=\sum u_{j} B^{j}, \mathbb{v}=\Sigma v_{p} B^{p}{ }_{p}$, where we put $\mathbb{1}:=\sum B_{p}^{p}$, 


$$
\text { 朋 } \oplus \mathrm{v}:=\sum_{j=1}^{l} u_{j} B_{j}^{j}+\sum_{k=1}^{r} v_{k} B_{k+l}^{k+l},
$$

and $\mathscr{A}_{\lambda+\rho, I+g}^{\Sigma \ltimes \mathrm{g}}:=\chi^{\Sigma \ltimes \mathrm{g}}{ }_{\lambda, l} \times \mathscr{D}^{\mathfrak{g}}=\Sigma_{\mu \in \Sigma \cdot \lambda} \chi^{\Sigma \times \mathrm{g}}{ }_{\mu, l}(1.2 .3(7 \sim 9), 1.3 .2(3))$.

Now (1) implies the desired identity

$$
\begin{aligned}
& \operatorname{ch}_{(2 l+1)(2 r+1)}^{\mathrm{Z}}\left(\tau D^{\mathrm{L}}(0)+\mathrm{L}(\mathrm{u})+\tau^{\prime} D^{\mathrm{P}}(0)+\mathrm{R}(\mathrm{v})\right) \\
& \quad=\Sigma_{Y \in \mathscr{Y}_{l, r}} \chi_{Y+\Lambda_{l}, 2 r+1}^{\left\langle\sigma_{01}\right\rangle \times \mathrm{D}(2 l+1)}(\tau, \mathrm{u}) \cdot \chi_{Y^{\dagger}+\Lambda_{r}, 2 l+1}^{\left\langle\sigma_{01}\right\rangle \times \mathrm{v}(2 r+1)}\left(\tau^{\prime}, \mathrm{v}\right),
\end{aligned}
$$

where we abbreviate $D^{\mathrm{L}}(0), D^{\mathrm{R}}(0)$ for $D^{\mathrm{g}_{\mathrm{L}}^{\wedge}}(0), D^{\mathrm{g}_{\mathrm{R}}}(0)$ respectively.

\subsection{Proof of Theorem 4.2 continued: in cases of $R^{2}=1 / 2$}

We need the following formulae for the cases of $h=1 / 2$.

Lemma 4.4. The following identities hold for $l, r, k \in \mathbb{Z}_{>0}, Y \in \mathscr{Y}_{l, r}, \lambda \in \hat{P}_{+}(r), \tau, u$, $u_{j} \in \mathbb{C}, \operatorname{Im} \tau>0$, 吸 $=\sum_{j=1}^{l} u_{j} X^{j}{ }_{j}$ and $\mathbb{1}:=\sum_{j=1}^{l} X_{j}^{j}(X=B, C, D$ according to the formula $(\mathbb{B}),(\mathbb{C}),(\mathbb{D}))$.

$$
\begin{gathered}
\theta_{k, l}^{\mathbb{Z}}\left(\tau, u-\frac{\tau}{2}\right)=\mathbb{e}[l u / 2-l \tau / 8] \theta_{k-l / 2, l}^{\mathbb{Z}}(\tau, u) \\
\chi_{Y, 2 r+1}^{\left\langle\sigma_{01}\right\rangle \times \mathfrak{o}(2 l+1)^{\wedge}}\left(\tau,-\mathbb{u}-\frac{\tau+1}{2} \mathbb{1}\right) \\
=(-1)^{[Y]} q^{l(2 r+1) / 8} \mathbb{e}\left[-\left(r+\frac{1}{2}\right) \Sigma u_{j}\right] \prod_{j} \frac{\theta\left(\tau, u_{j}\right)}{\hat{\theta}\left(\tau, u_{j}+\frac{1}{2}\right)} \chi_{Y^{c}, 2 r+1}^{\left\langle\sigma_{01}>\mathfrak{o}(2 l+1)^{\wedge}\right.}(\tau, \mathbb{u}) \\
\chi_{Y, r}^{\mathfrak{s p}(2 l)^{\wedge}}(\tau, \mathbb{u})=q^{l r / 4} \mathbb{e}\left[\Sigma r u_{j}\right] \chi_{Y^{c}, r}^{\mathfrak{s p}(2 l)^{\wedge}}\left(\tau,-\left(\frac{\tau}{2} \mathbb{1}+\mathbf{u}\right)\right) \\
\chi_{\lambda, r}^{\mathfrak{o}(2 l) \wedge}(\tau, \mathbb{u})=q^{l r / 8} \mathbb{e}\left[\frac{r}{2} \Sigma u_{j}\right] \chi_{\sigma_{w}(\lambda, r)}^{\mathfrak{o}(2 l) \wedge}\left(\tau,-\left(\frac{\tau}{2} \mathbb{1}+\mathbf{u}\right)\right)
\end{gathered}
$$

Proof. (A) follows from the definition (1.5.1(4)). As for (C) and (D), together with the Weyl group invariance, the diagram automorphism covariance

$$
\operatorname{ch}_{\mathbb{L}(\Lambda)}(h)=\operatorname{ch}_{\mathbb{L}\left(\sigma_{w}(\Lambda)\right)}\left(\sigma_{w}(h)\right)
$$

for the choice $w=\left(\begin{array}{ccccc}0 & \cdots & j & \cdots & l \\ l & \cdots & l-j & \cdots & 0\end{array}\right)$ yields these formulae. Use the formulae for $\sigma_{w}$ in $1.5 .4 \sim 5$.

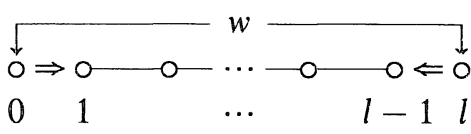

Case (C)

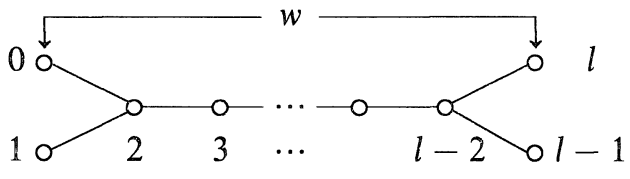

Case (D)

Proof of $(B)$ is complicated a little. We write the diagram automorphisms $(\S 1.3 .1$, $\S 1.5)$ of $D_{l}^{(1)}$ (resp. $B_{l}^{(1)}$ ) as $\sigma_{0}^{D}:=\sigma_{0,1}, \sigma_{l}^{D}:=\sigma_{l-1, l}, \sigma_{w}^{D}:=\sigma_{w}$ (resp. $\sigma_{0}^{B}:=\sigma_{0,1}$ ). Fixing $r \in \mathbb{Z}_{>0}$ and writing $(\lambda, k):=\lambda+k \hat{\Lambda}_{0}$, for $\sigma_{0}^{D}=\sigma_{0}^{D}$ and $\sigma_{l}^{D}$ we define $\sigma_{0}^{D}=\sigma_{0}^{D}$, 
$\stackrel{\circ}{l}_{l}^{D}: \mathfrak{h}^{* \mathfrak{o}(2 l)} \rightarrow \mathfrak{h}^{* \mathfrak{o}(2 l)}$ by

$$
\sigma_{\bullet}^{D}(\lambda, 2 r+2) \equiv\left(\sigma^{D}(\lambda), 2 r+2\right) \bmod \mathbb{C} \delta \quad\left(\lambda \in \mathfrak{h}^{* \mathfrak{D}(2 l)}\right),
$$

and also define $\stackrel{\circ}{\sigma}_{0}^{B}: \mathfrak{h}^{* \mathfrak{o}(2 l+1)} \rightarrow \mathfrak{h}^{* \mathfrak{o}(2 l+1)}$ by

$$
\sigma_{0}^{B}(\lambda, 2 r+1) \equiv\left(\sigma_{0}^{B}(\lambda), 2 r+1\right) \quad \bmod \mathbf{C} \delta \quad\left(\lambda \in \mathfrak{h}^{* 0(2 l+1)}\right) .
$$

We identify the weight spaces and lattice parts of affine Weyl groups for $\mathfrak{o}(2 l)$ and $\mathfrak{o}(2 l+1)$ by employing the expressions in $\S 1.5 .3$ and $\S 1.5 .5: \mathfrak{h}^{\mathfrak{o}(2 l)}=\mathfrak{h}^{\mathfrak{o}(2 l+1)}, M^{\mathfrak{o}(2 l)}=$ $M^{\mathrm{o}(2 l+1)}$. Then putting $\left[\Sigma \lambda_{j} \varepsilon_{j}\right]:=\Sigma \lambda_{j}$ and noting $\theta^{M^{\mathrm{o}(2 l)}}=\theta^{M^{\mathrm{o}(2 l+1)}}(1.2 .3(8))$, we deduce the disired identity as follows.

$$
\begin{aligned}
& \chi_{Y, 2 \boldsymbol{r}+1}^{\left\langle\sigma_{01}\right\rangle \propto \mathcal{D}(2 l+1)^{\wedge}}\left(\tau,-\mathbf{u}-\frac{\tau+1}{2} \mathbb{1}\right) \\
& =\chi_{Y, 2 r+1}^{\mathfrak{0}(2 l+1) \wedge}\left(\tau,-\mathbf{u}-\frac{\tau+1}{2} \mathbb{1}\right)+\chi_{\sigma_{0}^{B}(Y, 2 r+1)}^{\mathrm{o}(2 l+1) \wedge}\left(\tau,-\mathbf{u}-\frac{\tau+1}{2} \mathbb{1}\right) \\
& =\prod_{j=1}^{l} \frac{\eta(\tau)}{\theta\left(\tau,-u_{j}-\frac{\tau+1}{2}\right)}\left\{\chi_{(Y, 2 r+1)+\hat{\Lambda}_{l}}^{\mathrm{o}(2 l) \wedge}\left(\tau,-\mathbf{u}-\frac{\tau+1}{2} \mathbf{1}\right)\right. \\
& +\chi_{\sigma_{0}^{B}(Y, 2 r+1)+\hat{\Lambda}_{l}}^{\mathrm{o}(2 l)^{\wedge}}\left(\tau,-\mathbf{U}-\frac{\tau+1}{2} \mathbb{1}\right)-\chi_{\sigma_{l}^{D}\left((Y, 2 r+1)+\hat{\Lambda}_{l}\right)}^{\mathrm{o}(2 l)^{\wedge}}\left(\tau,-\mathbb{u}-\frac{\tau+1}{2} \mathbb{1}\right) \\
& \left.-\chi_{\sigma_{l}^{D}\left(\sigma_{0}^{B}(Y, 2 r+1)+\hat{\Lambda}_{l}\right.}^{\mathrm{o}(2 l)}\left(\tau,-\mathbf{u}-\frac{\tau+1}{2} \mathbb{1}\right)\right\} \\
& =\prod_{j=1}^{l} \frac{\eta(\tau)}{\theta\left(\tau,-u_{j}-\frac{\tau+1}{2}\right)} \times\left\{\sqrt{-1}^{-2\left[Y+\Lambda_{l}\right]} \chi_{Y+\Lambda_{l}, 2 r+2}^{(22)^{\wedge}}\left(\tau,-\mathbf{u}-\frac{\tau}{2} \mathbf{1}\right)\right. \\
& +\sqrt{-1}^{-2\left[\hat{\sigma}_{0}^{B}(Y)+\Lambda_{l}\right]} \chi_{\dot{\sigma}_{0}^{B}(Y)+\Lambda_{l}, 2 r+2}^{\mathfrak{o}(2 l)^{\wedge}}\left(\tau,-u-\frac{\tau}{2} \mathbb{1}\right) \\
& -\sqrt{-1}^{-2\left[\sigma_{l}^{D D}\left(Y+\Lambda_{l}\right)\right]} \chi_{\dot{\sigma}_{l}^{D}\left(Y+\Lambda_{l}\right), 2 r+2}^{\mathrm{o}(2 l)^{\wedge}}\left(\tau,-\mathbf{u}-\frac{\tau}{2} \mathbf{1}\right) \\
& \left.-\sqrt{-1}^{-2\left[\dot{\sigma}_{l}^{D}\left(\dot{\sigma}_{0}^{B}(Y)+\Lambda_{l}\right)\right]} \chi_{\dot{\sigma}_{l}^{D}\left(\sigma_{0}^{B}(Y)+\Lambda_{l}\right), 2 r+2}^{\mathcal{O}(2 l)}\left(\tau,-\mathbf{u}-\frac{\tau}{2} \mathbb{1}\right)\right\} \\
& =\prod_{j=1}^{l}\left(\frac{\eta(\tau)}{q^{-1 / 8} \mathbf{e}\left[-\frac{1}{2}\left(u_{j}-\frac{1}{2}\right)\right] \hat{\theta}\left(\tau,-u_{j}-\frac{1}{2}\right)}\right) \times q^{-l(2 r+2) / 8} \mathbf{e}\left[-(r+1) \Sigma u_{j}\right] \\
& \times\left\{\sqrt{-1}^{-2[Y]+l} \chi_{\sigma_{w}^{D}\left(Y+\Lambda_{l}, 2 r+2\right)}^{\mathrm{o}(2 l)}(\tau, \mathbf{u})+\sqrt{-1}^{-2[Y]-2-l} \chi_{\sigma_{w}^{D}\left(\dot{\sigma}_{0}^{B}(Y)+\Lambda_{l}, 2 r+2\right)}^{\left(2()^{\wedge}\right.}(\tau, \mathbf{u})\right.
\end{aligned}
$$

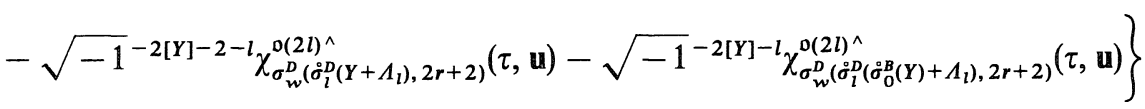




$$
\begin{aligned}
& =q^{-l(2 r+1) / 8} \mathbf{e}\left[-\frac{2 r+1}{2} \Sigma u_{j}\right](-1)^{[Y]} \prod_{j=1}^{l} \frac{\eta(\tau)}{\hat{\theta}\left(\tau, u_{j}+\frac{1}{2}\right)} \\
& \times\left\{\chi_{Y^{c}+\Lambda_{l}, 2 r+2}^{\mathrm{O}(2 l) \wedge}(\tau, \mathbf{u})-\chi_{\sigma_{l}^{D}\left(Y^{c}+\Lambda_{l}\right), 2 r+2}^{\mathrm{O}(2 l \wedge}(\tau, \mathbf{u})\right. \\
& \left.+\chi_{\dot{\sigma}_{0}^{B}\left(Y^{c}\right)+\Lambda_{l}, 2 r+2}^{\mathrm{o}\left(2 l \wedge^{\wedge}\right.}(\tau, \mathbf{u})-\chi_{\dot{\sigma}_{l}^{D}\left(\dot{\sigma}_{0}^{B}\left(Y^{c}\right)+\Lambda_{l}\right), 2 r+2}^{\mathrm{o}(2 l \wedge}(\tau, \mathbf{u})\right\} \\
& =q^{-l(2 r+1) / 8} \mathbf{e}\left[-\frac{2 r+1}{2} \Sigma u_{j}\right](-1)^{[Y]} \prod_{j=1}^{l} \frac{\theta\left(\tau, u_{j}\right)}{\hat{\theta}\left(\tau, u_{j}+\frac{1}{2}\right)} \\
& \times\left\{\chi_{Y^{c}, 2 r+1}^{\mathrm{o}(2 l+1) \wedge}(\tau, \mathrm{u})+\chi_{\sigma_{0}^{B}\left(Y^{c}\right), 2 r+1}^{\mathrm{o}(2 l+1) \wedge}(\tau, \mathbf{u})\right\}
\end{aligned}
$$

$$
=(\text { the right hand side of Lemma } 4.4(\mathrm{~B})) \text {. }
$$

Proof of Theorem 4.2, Case ÂÂ, $h=1 / 2$. Theorem 4.2 of Case ÂÂ $(h=0)$ means that the following identity holds,

$$
\begin{aligned}
& \prod_{\substack{1 \leq j \leq l \\
1 \leq p \leq r}} \frac{-i \theta\left(\tau, u_{j}+v_{p}+\frac{1}{2}\right)}{\eta(\tau)} \\
& =\Sigma_{Y \in \mathscr{O}_{l, r-1}} \chi_{Y,[Y]-l r / 2 ; r}^{\mathbf{Z}^{l} \times \mathrm{gl}^{\mathrm{I}(l)} \wedge}(\tau, \mathbf{u}) \chi_{t Y ; l}^{\mathrm{sl}(\boldsymbol{r}) \wedge}(\tau, \mathbf{v}) \\
& =\sum_{Y \in \mathscr{Y}_{l, r-1}} \sum_{1 \leq k \leq l} \frac{\theta_{[Y]+k r-l r / 2, l r}^{\mathbb{Z}}\left(\tau, \frac{[\mathbf{u}]}{l}\right)}{\eta(\tau)} \chi_{\sigma^{*}(Y ; r)}^{\mathfrak{I l}(l) \wedge}\left(\tau,\left.\mathbf{u}\right|_{\mathfrak{h}^{*}}\right) \chi_{t(Y ; l}^{\mathfrak{I}(r) \wedge}(\tau, \mathbf{v})
\end{aligned}
$$

where $\sigma=\sigma_{c y c}(1.5 .2(4)), \mathbf{u}=\Sigma u_{j} E_{j}^{j},[\mathbf{u}]:=\Sigma u_{j},\left.\quad \mathbf{u}\right|_{\mathfrak{h}^{*}}:=\mathbf{u}-\frac{[\mathbf{u}]}{l} \mathbf{1}_{l}$ and $\mathbf{v}=\Sigma v_{p} E_{p}^{p}$ is assumed to be $\operatorname{Tr} \mathbf{v}=\Sigma v_{p}=0$. Substituting $u_{j}$ by $u_{j}-\frac{\tau}{2}$, Lemma 4.4.(A) and the formula $\theta\left(\tau, u-\frac{\tau}{2}\right)=\mathbf{e}[u / 2-\tau / 8] \hat{\theta}(\tau, u)(1.5 .1(13))$ yield the desired identity for the Case $h=1 / 2$,

$$
\begin{aligned}
& \prod_{\substack{1 \leq j \leq l \\
1 \leq p \leq r}} \frac{\hat{\theta}\left(\tau, u_{j}+v_{p}+\frac{1}{2}\right)}{\eta(\tau)} \\
& =\Sigma_{Y \in \mathscr{O}} \mathscr{Y}_{l, r-1} \chi_{Y,[Y] ; r}^{Z^{l} \times \mathrm{g}(l) \wedge}(\tau, \mathbf{u}) \chi_{t Y ; l}^{\mathrm{gI}(r) \wedge}(\tau, \mathbf{v})
\end{aligned}
$$

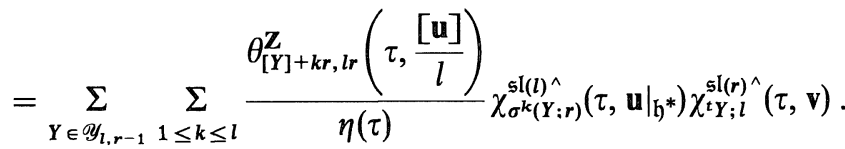


Proof of Theorem 4.2, Case $\widehat{\mathbb{C}} \hat{\mathrm{C}}, h=1 / 2$. Theorem 4.2 of Case $\widehat{\mathbb{C}} \hat{\mathbb{C}}(h=0)$, and Lemma $4.4(\mathrm{C})$ yield the required identity as follows:

(4) $\operatorname{ch}_{4 l r}^{\mathbb{Z}+1 / 2}\left(\tau D^{\text {Clif }}(0)+\mathbb{L}(\right.$ ld $\left.)+\mathbb{R}(\mathrm{v})\right)$

$$
\begin{aligned}
& =\prod_{1 \leq j \leq l, 1 \leq p \leq r}\left(\hat{\theta}\left(\tau, u_{j}+v_{p}+\frac{1}{2}\right) \hat{\theta}\left(\tau, u_{j}-v_{p}+\frac{1}{2}\right) \eta(\tau)^{-2}\right) \\
& =\prod_{j, p}\left(q^{1 / 4} \mathbb{e}\left[-u_{j}\right] \theta\left(\tau, u_{j}-\frac{\tau}{2}+v_{p}+\frac{1}{2}\right) \theta\left(\tau, u_{j}-\frac{\tau}{2}-v_{p}+\frac{1}{2}\right) \eta(\tau)^{-2}\right) \\
& =q^{l r / 4} \mathrm{e}\left[-\Sigma r u_{j}\right] \Sigma_{Y \in \mathscr{V}_{l, r}} \chi_{Y, r}^{\mathfrak{s p}(2 l)^{\wedge}}\left(\tau, \Sigma\left(u_{j}-\frac{\tau}{2}\right) C^{j}{ }_{j}\right) \chi_{Y^{+}, l}^{\mathfrak{s p}(2 r) \wedge}(\tau, \mathrm{v}) \\
& =\Sigma_{Y \in \mathscr{Y}_{l, r}} \chi_{Y^{c}, r}^{\mathrm{sp}(2 l)^{\wedge}}\left(\tau,-\Sigma u_{l+1-j} C^{j}{ }_{j}\right) \chi_{Y^{\dagger} l}^{\mathrm{sp}(2 r)^{\wedge}}(\tau, \mathrm{v}) \\
& =\Sigma_{Y \in \mathscr{Y}_{l, r}} \chi_{Y, r}^{\mathrm{sp}(2 l) \wedge}(\tau, \mathbb{Q}) \chi_{t Y, l}^{\mathrm{sp}(2 r)^{\wedge}}(\tau, \mathrm{v}) \quad\left(\text { 明 }=\Sigma_{1}^{l} u_{j} C^{j}{ }_{j}, \mathbb{v}=\Sigma_{1}^{r} v_{p} C^{p}{ }_{p}\right) .
\end{aligned}
$$

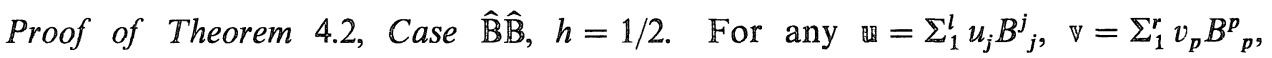
we have

(5) $\operatorname{ch}_{(2 l+1)(2 r+1)}^{\mathbb{Z}+1 / 2}\left(\tau D^{C l i f}(0)+\mathrm{L}(\mathbb{Q})+\mathrm{R}(\mathrm{v})\right)$

$$
\begin{aligned}
& =\frac{\eta(\tau)^{2}}{\eta\left(\frac{\tau}{2}\right) \eta(2 \tau)} \prod_{j=1}^{l} \frac{\hat{\theta}\left(\tau, u_{j}+\frac{1}{2}\right)}{\eta(\tau)} \prod_{p=1}^{r} \frac{\hat{\theta}\left(\tau, v_{p}+\frac{1}{2}\right)}{\eta(\tau)} \\
& \times \prod_{\substack{1 \leq j \leq l \\
1 \leq p \leq r}} \frac{\hat{\theta}\left(\tau, u_{j}+v_{p}+\frac{1}{2}\right) \hat{\theta}\left(\tau, u_{j}-v_{p}+\frac{1}{2}\right)}{\eta(\tau)^{2}} \\
& =(-1)^{l(r+1)} q^{l(2 r+1) / 8} \mathbb{e}\left[\left(r+\frac{1}{2}\right) \Sigma u_{j}\right] \prod_{j=1}^{l} \frac{\hat{\theta}\left(\tau,-u_{j}+\frac{1}{2}\right)}{\theta\left(\tau,-u_{j}\right)} \\
& \times \frac{\eta(\tau)^{2}}{\eta\left(\frac{\tau}{2}\right) \eta(2 \tau)} \prod_{j=1}^{l} \frac{\hat{\theta}\left(\tau,-\left(\frac{\tau+1}{2}+u_{j}\right)+\frac{1}{2}\right)}{\eta(\tau)} \prod_{p=1}^{r} \frac{\hat{\theta}\left(\tau, v_{p}+\frac{1}{2}\right)}{\eta(\tau)} \\
& \times \prod_{\substack{1 \leq j \leq l \\
1 \leq p \leq r}} \frac{\theta\left(\tau,-\left(\frac{\tau+1}{2}+u_{j}\right)-v_{p}\right) \theta\left(\tau,-\left(\frac{\tau+1}{2}+u_{j}\right)+v_{p}\right)}{\eta(\tau)^{2}} \\
& =(-1)^{l(r+1)} q^{l(2 r+1) / 8} \mathrm{e}\left[\left(r+\frac{1}{2}\right) \Sigma u_{j}\right] \prod_{j=1}^{l} \frac{\hat{\theta}\left(\tau,-u_{j}+\frac{1}{2}\right)}{\theta\left(\tau,-u_{j}\right)} \\
& \times \chi_{\hat{\Lambda}_{l+r}}^{\hat{2}_{l+2 r+1} \ltimes \mathrm{o}(2 l+2 r+1)^{\wedge}}\left(\tau,\left(-\frac{\tau+1}{2} \mathbb{B}-\mathbb{\mathbb { H }}\right) \oplus \mathbf{v}\right)
\end{aligned}
$$




$$
\begin{aligned}
& \times \mathscr{D}^{B_{l+r}^{(1)}}\left(\tau,-\left(\frac{\tau+1}{2} \mathbb{1}+\mathbf{u}\right) \oplus \mathbf{v}\right) / \mathscr{D}^{B_{l}^{(1)}}\left(\tau,-\frac{\tau+1}{2} \mathbb{1}+\mathbf{u}\right) \mathscr{D}_{r}^{B_{r}^{(1)}}(\tau, \mathbf{v}) \\
& =(-1)^{l(r+1)} q^{l(2 r+1) / 8} \mathbf{e}\left[\left(r+\frac{1}{2}\right) \Sigma u_{j}\right] \prod_{j=1}^{l} \frac{\hat{\theta}\left(\tau,-u_{j}+\frac{1}{2}\right)}{-\theta\left(\tau, u_{j}\right)} \\
& \times \Sigma_{Y \in \mathscr{Y}_{l, r}}(-1)^{\left[Y^{\dagger}\right]} \chi_{Y, 2 r+1}^{\sum_{2 l+1}^{\wedge} \times \mathbb{D}(2 l+1)^{\wedge}}\left(\tau,-\frac{\tau+1}{2} \mathbb{1}-\mathbf{u}\right) \chi_{\mathbf{Y}^{+}, 2 l+1}^{\Sigma_{2 r+1}^{\wedge} \times \mathbb{0}(2 r+1)^{\wedge}}(\tau, \mathbf{v}) \\
& \text { ([JM], Table II (6) } 7^{\circ} \text { for } \tilde{\Lambda}=\hat{\Lambda}_{0} \text { and } \hat{\Lambda}_{1} \text { ) }
\end{aligned}
$$

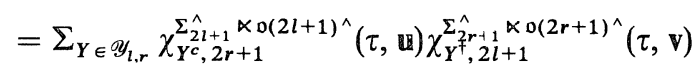

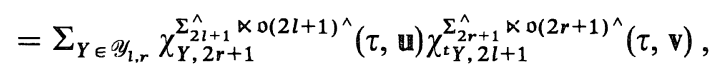

where $\mathscr{D}$ denotes the normalized denominator (1.5.3(3)).

Proof of Theorem 4.2, Case $\hat{\mathrm{D}} \hat{\mathrm{B}}, h=1 / 2 . \quad$ By the $h=0$ case and Lemma 4.4(D), we have

(6) $\operatorname{ch}_{2 l(2 r+1)}^{\mathrm{Z}+1 / 2}\left(\tau D^{\text {Clif }}(0)+\mathrm{L}(\mathrm{u})+\mathrm{R}(\mathrm{v})\right)$

$$
\begin{aligned}
& =\prod_{1 \leq j \leq l} \frac{\hat{\theta}\left(\tau, u_{j}+\frac{1}{2}\right)}{\eta(\tau)} \prod_{\substack{1 \leq j \leq l \\
1 \leq p \leq r}} \frac{\hat{\theta}\left(\tau, u_{j}+v_{p}+\frac{1}{2}\right) \hat{\theta}\left(\tau, u_{j}-v_{p}+\frac{1}{2}\right)}{\eta(\tau)^{2}} \\
& =\prod_{1 \leq j \leq l} q^{1 / 8} \mathbb{e}\left[-\frac{1}{2}\left(u_{j}+\frac{1}{2}\right)\right]^{\theta\left(\tau,-\left(u_{j}+\frac{\tau}{2}\right)+\frac{1}{2}\right)} \\
& \times \prod_{\substack{1 \leq j \leq l \\
1 \leq p \leq r}} q^{1 / 4} \mathbf{e}\left[u_{j}\right] \frac{-\theta\left(\tau,-\left(u_{j}+\frac{\tau}{2}\right)-v_{p}+\frac{1}{2}\right) \theta\left(\tau,-\left(u_{j}+\frac{\tau}{2}\right)+v_{p}+\frac{1}{2}\right)}{\eta(\tau)^{2}} \\
& =q^{l(2 r+1) / 8} \mathrm{e}\left[(2 r+1) \Sigma_{j} u_{j}\right]
\end{aligned}
$$

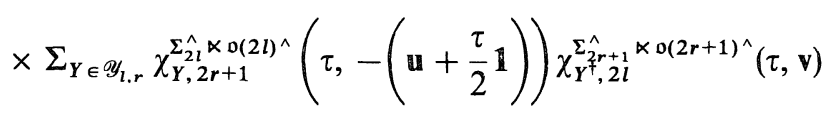

$$
\begin{aligned}
& =\Sigma_{Y \in \mathscr{Y}}{ }_{l, r} \chi_{Y, 2 r+1}^{\Sigma_{2 l}^{\wedge} \ltimes \mathcal{O}(2 l)^{\wedge}}(\tau, \mathbf{u}) \chi_{t, 2 l}^{\Sigma_{2 r+1}^{\wedge} \times \mathfrak{O}(2 r+1)^{\wedge}}(\tau, \mathbf{v})
\end{aligned}
$$

for any $\mathbf{u}=\Sigma_{1}^{l} u_{j} D_{j}^{j}$ and $\mathbf{v}=\Sigma_{1}^{r} v_{p} B_{p}^{p}$.

Proof of Theorem 4.2, Case $\hat{\mathrm{D}} \hat{\mathrm{D}}, h=1 / 2$. The result for the $h=0$ case and Lemma 4.4(D) yield the desired identity 
(7)

$$
\begin{aligned}
& \operatorname{ch}_{4 l r}^{\mathbf{Z}+1 / 2}\left(\tau D^{C l i f}(0)+\mathrm{L}(\mathbf{u})+\mathbf{R}(\mathbf{v})\right) \\
& =\prod_{1 \leq j \leq l, 1 \leq p \leq r}\left(\hat{\theta}\left(\tau, u_{j}+v_{p}+\frac{1}{2}\right) \hat{\theta}\left(\tau, u_{j}-v_{p}+\frac{1}{2}\right) \eta(\tau)^{-2}\right) \\
& =\prod_{j, p}\left(-q^{1 / 4} \mathbf{e}\left[u_{j}\right] \theta\left(\tau,-u_{j}-\frac{\tau}{2}-v_{p}+\frac{1}{2}\right) \theta\left(\tau,-u_{j}-\frac{\tau}{2}+v_{p}+\frac{1}{2}\right) \eta(\tau)^{-2}\right) \\
& =q^{l r / 4} \mathrm{e}\left[\Sigma r u_{j}\right] \Sigma_{Y \in \mathscr{Y}_{l, r}} \chi_{Y, 2 r}^{\Sigma_{2 l}^{\wedge} \times \mathrm{o}(2 l)^{\wedge}}\left(\tau,-\Sigma\left(u_{j}+\frac{\tau}{2}\right) D_{j}^{j}\right) \chi_{Y, 2 l}^{\Sigma_{2}^{\wedge} \times \mathrm{o}(2 r)^{\wedge}}(\tau, \mathrm{v}) \\
& =\Sigma_{Y \in \mathscr{Y} l, r} \chi_{Y c, 2 r}^{\Sigma_{2 l}^{\wedge} \times \mathrm{o}(2 l) \wedge}(\tau, \mathbf{u}) \chi_{Y^{\ddagger}, 2 l}^{\Sigma_{2}^{\wedge} \times \mathrm{o}(2 r)^{\wedge}}(\tau, \mathrm{v}),
\end{aligned}
$$

where $\mathbf{u}=\Sigma_{1}^{l} u_{j} D_{j}^{j}, \mathbf{v}=\Sigma_{1}^{r} v_{p} D_{p}^{p}$.

\section{§5. An Application to the Duality of Branching Rules}

5.0. In the context of seesaw pair $(\S 5.1,[\mathrm{Ku}])$, we give a simple explanation and generalizations of Jimbo-Miwa's duality in $\$ 5.2 \sim 5.4$. In $\S 5.4$ we find a relation between the diagram automorphisms and the inclusion $\mathfrak{g} \otimes \mathbb{C}\left[t, t^{-1}\right] \oplus \mathbb{C} c \supset \mathfrak{g} \otimes \mathbb{C}\left[t^{m}, t^{-m}\right] \oplus \mathbb{C}$.

\subsection{Seesaw pairs and dualities}

Definition 5.1 (Seesaw pair $[\mathrm{Ku}]$ ). Suppose that there are two dual pairs $(\S 0.1)$ $\left(A_{1}, A_{1}^{\prime}\right)$ and $\left(A_{2}, A_{2}^{\prime}\right)$ acting on the same module $V$. After Kudla $[\mathrm{Ku}]$, we say the pair $\left(\left(A_{1}, A_{1}^{\prime}\right),\left(A_{2}, A_{2}^{\prime}\right)\right)$ of dual pairs form a seesaw pair if they satisfy the inclusion relations

$$
A_{1} \supset A_{2} \quad \text { and (hence) } A_{1}^{\prime} \subset A_{2}^{\prime}
$$

in End $V$. We will write this situation as follows.

$$
\begin{array}{lll}
A_{1} \supset A_{2} & \\
+ & & + \\
A_{1}^{\prime} & \subset & A_{2}^{\prime} .
\end{array}
$$

As we shall see below, one seesaw pair provides us one duality of branching rules, i.e., an isomorphism between branching modules.

By $\operatorname{Irr} A$ we denote the totality of isomorphism classes of simple $A$-modules, and by $\mathbf{L}_{\lambda}^{A}$ the representative for $\lambda \in \operatorname{Irr} A$. Recall the definition

$$
\mathbb{B}\left(A_{1} \supset A_{2}\right)_{\Lambda}^{\lambda}:=\operatorname{Hom}_{A_{2}}\left(\mathbb{L}_{\lambda}^{A_{2}}, \mathbb{L}_{\Lambda}^{A_{1}}\right)
$$

of a branching module $\left(\Lambda \in \operatorname{Irr} A_{1}, \lambda \in \operatorname{Irr} A_{2} ; 0.2(1), 1.2 .4(3)\right)$.

Proposition 5.1. Assume the complete reducibility in the above situation (1), and write the irreducible decompositions of $V$ as a $A_{j} \oplus A_{j}^{\prime}$-module $(j=1,2)$ as

$$
V \simeq \bigoplus_{l \in I_{j}} \mathbb{L}_{\lambda_{j}(l)}^{A_{j}{ }^{\prime}} \otimes \mathbb{L}_{\lambda_{j}^{\prime}(l)}^{A_{j}^{\prime}},
$$


where $I_{j} \quad$ (resp. $\quad \lambda_{j}: I_{j} \rightarrow \operatorname{Irr} A_{j}, \quad \lambda_{j}^{\prime}: I_{j} \rightarrow \operatorname{Irr} A_{j}^{\prime}$ ) are suitable index sets (resp. suitable injections).

Then for $\alpha \in I_{1}$ and $\beta \in I_{2}$ we have the following isomorphism between the branching modules (“duality"):

$$
\mathbf{B}\left(A_{1} \supset A_{2}\right)_{\lambda_{1}(\alpha)}^{\lambda_{2}(\beta)} \simeq \mathbf{B}\left(A_{2}^{\prime} \supset A_{1}^{\prime}\right)_{\lambda_{2}^{\prime}(\beta)}^{\lambda_{1}^{\prime}(\alpha)}
$$

Proof. Consider $V$ as a $A_{1} \oplus A_{1}^{\prime}$ - (resp. $A_{2} \oplus A_{2}^{\prime}$ ) module, and then decompose it with respect to the subalgebra $A_{2} \oplus A_{1}^{\prime} \subset A_{1} \oplus A_{1}^{\prime}$ (resp. $\subset A_{2} \oplus A_{2}^{\prime}$ ). Under the assumption of the complete reducibility we respectively obtain the followings:

$$
\begin{aligned}
V & \simeq \bigoplus_{\alpha \in I_{1}} \mathbf{L}_{\lambda_{1}(\alpha)}^{A_{1}} \otimes \mathbf{L}_{\lambda_{1}^{\prime}(\alpha)}^{A_{1}^{\prime}} \\
& \simeq \bigoplus_{\alpha \in I_{1}} \bigoplus_{\mu \in \operatorname{Irr} A_{2}}\left(\mathbf{B}\left(A_{1} \supset A_{2}\right)_{\lambda_{1}(\alpha)}^{\mu} \otimes \mathbf{L}_{\mu}^{A_{2}}\right) \otimes \mathbf{L}_{\lambda_{1}^{\prime}(\alpha)}^{A_{1}^{\prime}}
\end{aligned}
$$

and

$$
\begin{aligned}
V & \simeq \bigoplus_{\beta \in I_{2}} \mathbf{L}_{\lambda_{2}(\beta)}^{A_{2}} \otimes \mathbf{L}_{\lambda_{2}^{\prime}(\beta)}^{A_{2}^{\prime}} \\
& \simeq \bigoplus_{\beta \in I_{2}} \bigoplus_{v \in \operatorname{Irr} A_{1}^{\prime}} \mathbf{L}_{\lambda_{2}(\beta)}^{A_{2}(\beta)} \otimes\left(\mathbf{B}\left(A_{2}^{\prime} \supset A_{1}^{\prime}\right)_{\lambda_{2}^{\prime}(\beta)}^{v} \otimes \mathbf{L}_{v}^{g_{1}^{\prime}}\right) .
\end{aligned}
$$

Now we compare the coefficients and get

$$
\begin{aligned}
\mathbf{B}\left(A_{1} \supset A_{2}\right)_{\lambda_{1}(\alpha)}^{\mu} \otimes \Delta_{\lambda_{1}^{\prime}(\alpha)}^{v} & \simeq \mathbf{B}\left(\text { End } V \supset A_{2} \oplus A_{1}^{\prime}\right)_{V}^{\mu, v} \\
& \simeq \Delta_{\lambda_{2}(\beta)}^{\mu} \otimes \mathbf{B}\left(\mathfrak{g}_{2}^{\prime} \supset \mathfrak{g}_{1}^{\prime}\right)_{\lambda_{2}^{\prime}(\beta)}^{v},
\end{aligned}
$$

where $\Delta_{\lambda}^{\mu}:=\left\{\begin{array}{ll}\mathbf{C} & (\text { if } \lambda=\mu) \\ 0 & \text { (otherwise) }\end{array}\right.$. This implies the proposition.

\subsection{Finite dimensional case}

We will present some typical ones of seesaw pairs on spinors and the corresponding dualities.

From now on, we abbreviate the dual pair $\left(\mathbf{C}[\Sigma] \ltimes U(\mathfrak{g}), \mathbf{C}\left[\Sigma^{\prime}\right] \ltimes U\left(\mathfrak{g}^{\prime}\right)\right)$ as $\left(\Sigma \ltimes \mathfrak{g}, \Sigma^{\prime} \ltimes \mathfrak{g}^{\prime}\right)$ (or $\left(\mathfrak{g}, \mathfrak{g}^{\prime}\right)$ : when $\Sigma$ and $\Sigma^{\prime}$ are trivial).

(a0). $(\mathfrak{g l}(l), \mathfrak{s l}(2 r))$ and $(\mathfrak{s p}(2 l), \mathfrak{s p}(2 r))$ on $\bigwedge\left(W_{4 l r}^{-}\right)$.

Theorem 3.2 says that these two pairs form dual pairs in $C\left(W_{4 l r}\right) \simeq$ End $\bigwedge\left(W_{4 l r}^{-}\right)$. Moreover they form a seesaw pair:

$$
\begin{array}{ccc}
\mathfrak{s l}(2 r) & \supset & \mathfrak{s p}(2 r) \\
+ & + \\
\mathfrak{g l}(l) & \subset & \mathfrak{s p}(2 l) .
\end{array}
$$

After the notation in 3.2 these inclusion relations are realized by the following embeddings (see $[\mathrm{JM} \S 1,(1.3)$ and (1.4)]): 
(2) $\quad \mathfrak{s l}(2 r) \simeq\left\{\sum_{j=1}^{l} \mathbb{C}: \psi^{p, j} \psi_{q, j}:\right\}_{p, q= \pm 1 \cdots \pm r}$

$$
\supset\left\{\sum_{j=1}^{l} \mathbb{C}: \psi^{p, j} \psi_{q, j}-\operatorname{sgn}(p q) \psi^{-q, j} \psi_{-p, j}:\right\}_{p, q= \pm 1 \cdots \pm r} \simeq \mathfrak{s p}(2 r),
$$

(3) $\operatorname{gl}(l) \simeq\left\{\Sigma_{p=-r}^{r} \mathbb{C}: \psi^{p, i} \psi_{p, j}:\right\}_{i, j=1 \cdots l}$

$$
\subset\left\{\sum_{p=1}^{r} \mathbb{C}: \psi^{p, i} \psi_{p, j}-\operatorname{sgn}(i j) \psi^{p,-j} \psi_{p,-i}:\right\}_{i, j= \pm 1 \cdots \pm l} \simeq \mathfrak{s p}(2 l) .
$$

Associated to this seesaw pair, we obtain the following duality as a direct consequence of Proposition 5.1.

Corollary 5.22 ([JM, Proposition 1.3]).

For $Y \in \mathscr{Y}_{l, 2 r}$ and $y \in \mathscr{Y}_{l, r}$, we have

$$
\mathbb{B}(\mathfrak{s l}(2 r) \supset \mathfrak{s p}(2 r))_{Y^{\dagger}}^{y^{\dagger}} \simeq \mathbb{B}(\mathfrak{s p}(2 l) \supset \mathfrak{g l}(l))_{y}^{Y} .
$$

(b0). Next we consider the pairs

$$
(\mathfrak{g l}(m+n), \mathfrak{s l}(l)) \quad \text { and } \quad(\mathfrak{g l}(m) \oplus \mathfrak{g l}(n), \mathfrak{s l}(l) \oplus \mathfrak{s l}(l))
$$

acting on $\bigwedge\left(W_{2 l(m+n)}^{-}\right) \simeq \bigwedge\left(W_{2 l m}^{-}\right) \otimes \bigwedge\left(W_{2 l n}^{-}\right)$. Each of them forms a dual pair on $\bigwedge\left(W_{2 l(m+n)}^{-}\right)$, as for the latter pair which fact is easily seen as follows.

$$
\begin{aligned}
& \bigwedge\left(W_{2 l(m+n)}^{-}\right) \simeq\left(\oplus_{Y \in \mathscr{Y}_{m, l}} \mathbb{L}^{\mathrm{gl}(m)}\left(Y, \frac{m \cdot l}{2}-|Y|\right) \otimes \mathbb{L}^{\mathrm{sl}(l)}\left({ }^{t} Y\right)\right) \\
& \otimes\left(\bigoplus_{y \in \mathscr{Y}_{n, l}} \mathbb{L}^{\mathrm{gl}(n)}\left(y, \frac{n \cdot l}{2}-|y|\right) \otimes \mathbb{L}^{\mathfrak{s I}(l)}\left({ }^{t} y\right)\right) \\
& \simeq \bigoplus_{(Y, y) \in \mathscr{Y}_{m, l} \times \mathscr{Y}_{n, l}}\left(\mathbb{L}^{\mathrm{gl}(m)}\left(Y, \frac{m \cdot l}{2}-|Y|\right) \otimes \mathbb{L}^{\mathrm{gl}(n)}\left(y, \frac{n \cdot l}{2}-|y|\right)\right) \\
& \otimes\left(\mathbb{L}^{\mathrm{sl}(l)}\left({ }^{t} Y\right) \otimes \mathbb{L}^{\mathrm{sl}(l)}\left({ }^{t} y\right)\right) .
\end{aligned}
$$

They form a seesaw pair

$$
\begin{array}{rcc}
\mathfrak{g l}(m+n) & \supset \mathfrak{g l}(m) & \oplus \mathfrak{g l}(n) \\
+ & + \\
\mathfrak{s l}(l) & \subset \operatorname{sl}(l) & \oplus \mathfrak{s l}(l) \quad \text { (: diagonal embedding) }
\end{array}
$$

and Proposition 5.1 yields

Corollary 5.2b. For $y \in \mathscr{Y}_{l, m}, y^{\prime} \in \mathscr{Y}_{l, n}$ and $Y \in \mathscr{Y}_{l, m+n}$, the following isomorphism holds.

$$
\mathbb{B}(\mathfrak{g l}(m+n) \supset \mathfrak{g l}(m) \oplus \mathfrak{g l}(n))_{t_{Y}}^{t_{Y}, t_{y^{\prime}}} \simeq \mathbb{B}(\mathfrak{s l}(l) \oplus \mathfrak{s l}(l) \supset \mathfrak{s l}(l))_{y, y^{\prime}}^{Y}
$$

We present other examples of seesaw pairs in Tables 5.2a and 2b. If a seesaw pair include $\mathfrak{o}(2 n)$ as its constituents, then we have to take the diagram automorphism $\Sigma_{2 n}=\left\langle\sigma_{n-1, n}\right\rangle$ into consideration. 
Table 5.2a

$$
\mathfrak{s l}(r) \supset \Sigma_{r} \ltimes \mathfrak{o}(r)
$$

(a1).

$$
\begin{gathered}
+\quad+ \\
\operatorname{gl}(l) \subset \Sigma_{2 l} \ltimes \mathfrak{p}(2 l) \\
\Sigma_{4 r} \ltimes \mathfrak{b}(4 r) \supset \mathfrak{s p}(2 r)
\end{gathered}
$$$$
\text { (on } \left.\bigwedge\left(W_{2 l r}^{-}\right)\right)
$$

(a2).

(a3).

(a4).

$$
\begin{array}{cc}
+ & + \\
\Sigma_{l} \ltimes \mathfrak{o}(l) & \subset \mathfrak{s p}(2 l) \\
\mathfrak{s l}(l m) \supset \bigoplus^{m} \mathfrak{s l}(l) \supset \operatorname{sI}(l)
\end{array}
$$

$+\quad+$ (on $\bigwedge\left(W_{2 l m n}^{-}\right)$)

$$
\begin{aligned}
\mathfrak{g l}(n) & \subset \bigoplus^{m} \mathfrak{g l}(n) \subset \mathfrak{g l}(m n) \\
\mathfrak{s p}(2 l m) & \supset \bigoplus^{m} \mathfrak{s p}(2 l) \supset \mathfrak{s p}(2 l)
\end{aligned}
$$

$$
\begin{array}{ccc}
+ & + & + \\
\mathfrak{s p}(2 n) & \subset \bigoplus^{m} \mathfrak{s p}(2 n) \subset \mathfrak{s p}(2 m n) \\
\Sigma_{l m} \ltimes \mathfrak{o}(l m) \supset \bigoplus^{m} \Sigma_{l} \ltimes \mathfrak{p}(l) \supset \quad \Sigma_{l} \ltimes \mathfrak{o}(l)
\end{array}
$$

(a5).

$$
\Sigma_{n} \ltimes \mathfrak{o}(n) \subset \bigoplus^{m} \Sigma_{n} \ltimes \mathfrak{o}(n) \subset \Sigma_{m n} \ltimes \mathfrak{o}(m n)
$$

(on $\bigwedge\left(W_{4 l r}^{-}\right)$)

(on $\left.\bigwedge\left(W_{4 l m n}^{-}\right)\right)$

\section{Table 5.2b}

$$
\begin{aligned}
& \mathfrak{s p}(2 m+2 n) \supset \mathfrak{s p}(2 m) \oplus \mathfrak{s p}(2 n) \\
& \mathfrak{s p}(2 l) \quad \subset \mathfrak{s p}(2 l) \oplus \mathfrak{s p}(2 l) \\
& \text { (on } \left.\bigwedge\left(W_{4 l(m+n)}^{-}\right) \simeq \bigwedge\left(W_{4 l m}^{-}\right) \otimes \bigwedge\left(W_{4 l n}^{-}\right)\right) \\
& \Sigma_{M+N} \ltimes \mathfrak{o}(M+N) \supset\left(\Sigma_{M} \ltimes \mathfrak{o}(M)\right) \oplus\left(\Sigma_{N} \ltimes \mathfrak{o}(N)\right) \\
& \Sigma_{L} \ltimes \mathfrak{o}(L) \quad \subset\left(\Sigma_{L} \ltimes \mathfrak{o}(L)\right) \oplus\left(\Sigma_{L} \ltimes \mathfrak{o}(L)\right) \\
& \text { (on } \left.\bigwedge\left(W_{L(M+N)}^{-}\right) \simeq \bigwedge\left(W_{L M}^{-}\right) \otimes \bigwedge\left(W_{L N}^{-}\right)\right)
\end{aligned}
$$

(b1).

(b2).

Notation for Table 5.2. Here we put $\Sigma_{2 n}:=\left\langle\sigma_{n-1, n}\right\rangle(1.3 .1,1.4 .5), \Sigma_{2 n+1}:=\{i d\}$.

Remark 5.2. The versions of Corollary 5.2b for the above seesaw pairs show that the works [Ko], [KoT1] and [KoT2] are mutually in the same depth in the sence that if we know the branching rules of the tensor product representation ([Ko, Theorem 3.1], [KoT1, Cor. 2.5.3]), it also means that we know the branching rules with respect to the restriction to the reductive subgroups of maximal rank ([KoT2, Theorem 2.5]). For any type of classical Lie algebras Koike and Terada generalized there the celebrated Littlewood-Richardson rule, which gives an exact algorithm to compute the multiplicity $\operatorname{dim} \mathbb{B}(\mathfrak{g l}(n) \oplus \mathfrak{g l}(n) \supset \mathfrak{g l}(n))_{\mu}^{\lambda}$ in terms of Young diagrams. To consider their affine versions will be an interesting problem. 


\subsection{Affine case (1)}

The seesaw pairs we present in $\S 5.2$ have their affine versions. They explain so called "rank-level dualities" concerning with the coset Virasoro modules.

For example, as an affine version for $(\mathrm{a} 0)$ there is a seesaw pair

$$
\begin{array}{lrl}
\mathfrak{s l}(2 r)^{\wedge} & \supset & \mathfrak{s p}(2 r)^{\wedge} \\
(\text { level } l) & (\text { level } l)
\end{array}
$$

$(\mathrm{a} 0)^{\wedge}$.

$$
\begin{array}{ccc}
+ & + & \left(\text { on } \bigwedge\left(W_{4 l r}^{\mathbb{Z}+h,-}\right)\right) . \\
\mathbb{Z}^{l} \propto \operatorname{gl}(l)^{\wedge} & \subset & \operatorname{sp}(2 l)^{\wedge} \\
(\text { level 2r) } & (\text { level } r) &
\end{array}
$$

Here the inclusions $\mathfrak{s l}(2 r)^{\wedge} \supset \mathfrak{s p}(2 r)^{\wedge}$ and $\mathfrak{g l}(l)^{\wedge} \subset \mathfrak{s p}(2 l)^{\wedge}$ are what induced by 5.2(2) and 5.2(3) respectively, and $\mathbb{Z}^{l}$ stands for the lattice part $M$ of the Weyl group of $\mathfrak{s p}(2 l)^{\wedge}(1.5 .4(2))$, which act on $\mathfrak{g l}(l)^{\wedge} \subset \mathfrak{s p}(2 l)^{\wedge}$ as the automorphism given by $1.3 .4(8)$. This seesaw pair yields

Corollary 5.3a. (i) For $Y, y \in \mathscr{Y}_{r, l}$, we have the isomorphisms of coset $\mathscr{V}_{i \text {-modules }}$

$$
\begin{aligned}
\mathbb{B}\left(\mathfrak{s l}(2 r)^{\wedge} \supset \mathfrak{s p}(2 r)^{\wedge}\right)_{(Y ; l)}^{(y ; l)} & \simeq \mathbf{B}\left(\mathfrak{s p}(2 l)^{\wedge} \supset \mathbb{Z}^{l} \ltimes \mathfrak{g l}(l)^{\wedge}\right)_{(t y ; r)}^{(t Y,[Y] ; r)} \\
& \simeq \mathbb{B}\left(\mathfrak{s p}(2 l)^{\wedge} \supset \mathbb{Z}^{l} \ltimes \mathfrak{g l}(l)^{\wedge}\right)_{\left(y^{\dagger} ; r\right)}^{\left(Y^{+},[Y]-l r / 2 ; r\right)} .
\end{aligned}
$$

(ii) For $0 \leq p \leq r$, let $\hat{\Lambda}_{p}=\Lambda_{p}+\hat{\Lambda}_{0}$ be the fundamental weight of $\mathfrak{s p}(2 r)^{\wedge} \simeq \mathfrak{g}\left(\mathbb{C}_{r}^{(1)}\right)$ (1.2.1(6)). Then the $\mathfrak{s p}(2 r)^{\wedge}$-module $\mathbb{L}^{\mathfrak{s p}(2 r)^{\wedge}}\left(\hat{\Lambda}_{p}\right)=\mathbb{L}^{\mathfrak{s p}(2 r)^{\wedge}}\left(\Lambda_{p} ; 1\right)$ is irreducible as a $\mathbb{Z}^{l} \times \mathfrak{g l}(l)^{\wedge}$-module.

Proof. (i) The complete reducibility for the above restrictions are ensured by Lemmata 1.2(i) and 1.3.4(i) (at this point we need §1.3). Hence in view of Proposition

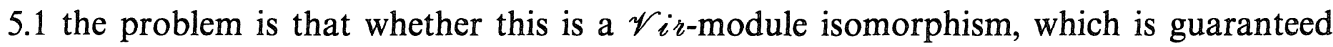

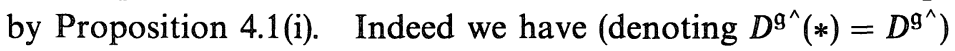

$$
\begin{aligned}
& D^{\mathrm{sl}(2 \boldsymbol{r})^{\wedge}}-D^{\mathfrak{s p}(2 \boldsymbol{r})^{\wedge}}=\left(D^{\mathrm{gl}(l)^{\wedge}}+D^{\mathrm{Sl}(2 r)^{\wedge}}\right)-\left(D^{\mathrm{gl}(l)^{\wedge}}+D^{\mathfrak{s p}(2 r)^{\wedge}}\right) \\
& =D^{\mathrm{o}(4 l r)^{\wedge}}-\left(D^{\mathrm{g} \mathrm{g}(l)^{\wedge}}+D^{\mathrm{sp}(2 r)^{\wedge}}\right) \\
& =\left(D^{\mathfrak{s p}(2 l)^{\wedge}}+D^{\mathfrak{s p}(2 r)^{\wedge}}\right)-\left(D^{\mathfrak{g l}(l)^{\wedge}}+D^{\mathfrak{s p}(2 r)^{\wedge}}\right) \\
& =D^{\mathfrak{s p}(2 l)^{\wedge}}-D^{\mathrm{gl}(l)^{\wedge}} \text {. }
\end{aligned}
$$

(ii) Let $r=1$ in (i). Noting $\mathfrak{s p}(2) \simeq \mathfrak{s l}(2)$, we have

$$
\begin{aligned}
\Delta_{Y}^{y} & =\mathbf{B}\left(\mathfrak{s l}(2)^{\wedge} \supset \mathfrak{s p}(2)^{\wedge}\right)_{(Y ; l)}^{(y ; l)} \\
& \simeq \mathbf{B}\left(\mathfrak{s p}(2 l)^{\wedge} \supset \mathbb{Z}^{l} \ltimes \mathfrak{g l}(l)^{\wedge}\right)_{\left(t_{y} ; 1\right)}^{(t Y,[Y] ; 1)}
\end{aligned}
$$

for any $Y, y \in \mathscr{Y}_{1, l}$ (see 5.1(7) for $\left.\Delta_{Y}^{y}\right)$. Since $\left({ }^{t} y ; 1\right)={ }^{t} y+\hat{\Lambda}_{0}\left(y \in \mathscr{Y}_{1, l}\right)$ exausts the fundamental weights $\hat{\Lambda}_{p}(0 \leq p \leq l)$, we conclude (ii).

See Table 5.3 for other examples of affine versions. As in the above Corollary, the complete reducibility for the seesaw pair there is ensured by Lemmata 1.2, 1.3.2 and 1.3.4. 
The duality associated to the seesaw pair (b1)^ is the following, whose special case $n=1$ is known by $[\mathrm{KW}]$ and $[\mathrm{Ya}]$.

Corollary 5.3b. For $y \in \mathscr{Y}_{l, n}, y^{\prime} \in \mathscr{Y}_{m, n}, Y \in \mathscr{Y}_{l+m, n}$ and $h=0$ or $1 / 2$, we have an isomorphism of coset $\mathscr{V}_{i \imath \text {-modules }}$

$$
\begin{aligned}
& \mathbf{B}\left(\mathfrak{s p}(2 n)^{\wedge} \oplus \mathfrak{s p}(2 n)^{\wedge} \supset \mathfrak{s p}(2 n)^{\wedge}\right)_{\left(y^{h}, l\right),\left(y^{\prime}, m\right)}^{\left(Y^{h}, l+m\right)} \\
& \quad \simeq \mathbf{B}\left(\mathfrak{s p}(2 l+2 m)^{\wedge} \supset \mathfrak{s p}(2 l)^{\wedge} \oplus \mathfrak{s p}(2 m)^{\wedge}\right)_{(Y, n)}^{(y, n),\left(y^{\prime}, n\right)} .
\end{aligned}
$$

where we put $y^{h}:=y^{\dagger}(h=0) ;{ }^{t} y(h=1 / 2)$, etc.

\section{Table 5.3a}

$$
\mathfrak{s l}(r)^{\wedge} \supset \Sigma_{r}^{\wedge} \times \mathfrak{o}(r)^{\wedge}
$$

$$
\begin{aligned}
& +\quad+\quad \text { (on } \bigwedge\left(W_{2 l r}^{\mathbf{Z}+h,-}\right) \text { ) } \\
& \mathbf{Z}^{l} \times \mathrm{gl}(l)^{\wedge} \subset \Sigma_{\hat{2 l}}^{\wedge} \times \mathfrak{o}(2 l)^{\wedge} \\
& \Sigma_{4 r}^{\wedge} \times \mathfrak{o}(4 r)^{\wedge} \supset \mathfrak{s p}(2 r)^{\wedge} \\
& \text { (a2)^. } \\
& \begin{array}{cc}
+ & + \\
\Sigma_{l}^{\wedge} \ltimes \mathfrak{o}(l)^{\wedge} & \subset \mathfrak{s p}(2 l)^{\wedge}
\end{array}
\end{aligned}
$$

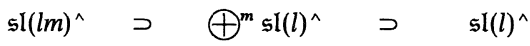

$$
\begin{aligned}
& \begin{array}{ccc}
+ & + & + \\
\mathbf{Z}^{n} \times \operatorname{gl}(n)^{\wedge} \subset \bigoplus^{m} \mathbf{Z}^{n} \times \operatorname{gl}(n)^{\wedge} \subset \mathbf{Z}^{m n} \times \operatorname{gl}(m n)^{\wedge}
\end{array} \\
& \mathfrak{s p}(2 l m)^{\wedge} \supset \bigoplus^{m} \mathfrak{s p}(2 l)^{\wedge} \supset \mathfrak{s p}(2 l)^{\wedge} \\
& \begin{array}{ccc}
+ & + & + \\
\mathfrak{s p}(2 n)^{\wedge} & \subset \bigoplus^{m} \mathfrak{s p}(2 n)^{\wedge} & \subset \mathfrak{s p}(2 l m n)^{\wedge}
\end{array} \\
& \Sigma_{l m}^{\wedge} \ltimes \mathfrak{o}(l m)^{\wedge} \dot{\jmath} \oplus^{m} \Sigma_{\hat{l}} \ltimes \mathfrak{o}(l)^{\wedge} \supset \Sigma_{\imath}^{\wedge} \times \mathfrak{o}(l)^{\wedge} \\
& \Sigma_{n}^{\wedge} \times \mathfrak{o}(n)^{\wedge} \subset \bigoplus^{m} \Sigma_{n}^{\wedge} \times \mathfrak{o}(n)^{\wedge} \subset \Sigma_{m n}^{\wedge} \times \mathfrak{o}(m n)^{\wedge}
\end{aligned}
$$

\section{Table 5.3b}

(b0)^.

$$
\mathbf{Z}^{m+n} \times \operatorname{gl}(m+n)^{\wedge} \supset\left(\mathbf{Z}^{m} \times \operatorname{gl}(m)^{\wedge}\right) \oplus\left(\mathbf{Z}^{n} \times \operatorname{gl}(n)^{\wedge}\right)
$$

(b)

(b1)^.

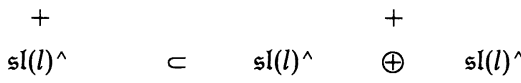

$$
\begin{aligned}
& \text { (on } \left.\bigwedge\left(W_{2 l(m+n)}^{\mathbf{Z}+h,-}\right) \simeq \bigwedge\left(W_{2 l m}^{\mathbf{Z}+h,-}\right) \otimes \bigwedge\left(W_{2 l n}^{\mathbf{Z}+h,-}\right)\right) \\
& \mathfrak{s p}(2 m+2 n)^{\wedge} \supset \mathfrak{s p}(2 m)^{\wedge} \oplus \mathfrak{s p}(2 n)^{\wedge} \\
& \mathfrak{s p}(2 l)^{\wedge} \quad \subset \mathfrak{s p}(2 l)^{\wedge} \oplus \mathfrak{s p}(2 l)^{\wedge} \\
& \text { (on } \left.\bigwedge\left(W_{4 l(m+n)}^{\mathbf{Z}+h_{-}-}\right) \simeq \bigwedge\left(W_{4 l m}^{\mathbf{Z}+h_{-}-}\right) \otimes \bigwedge\left(W_{4 l n}^{\mathbf{Z}+h_{,-}}\right)\right) \\
& \Sigma_{\hat{M}+N} \times \mathfrak{o}(M+N)^{\wedge} \supset\left(\Sigma_{\hat{M}} \ltimes \mathfrak{o}(M)^{\wedge}\right) \oplus\left(\Sigma_{\hat{N}} \times \mathfrak{o}(N)^{\wedge}\right) \\
& \Sigma_{\hat{L}}^{\wedge} \times \mathfrak{o}(L)^{\wedge} \quad \subset\left(\Sigma_{\hat{L}} \ltimes \mathfrak{o}(L)^{\wedge}\right) \oplus\left(\Sigma_{\hat{L}} \times \mathfrak{o}(L)^{\wedge}\right) \\
& \text { (on } \left.\bigwedge\left(W_{L(M+N)}^{\mathbf{Z}+h,-}\right) \simeq \bigwedge\left(W_{L M}^{\mathbf{Z}+h,-}\right) \otimes \bigwedge\left(W_{L N}^{\mathbf{Z}+h,-}\right)\right)
\end{aligned}
$$

(b2)^.

Notation for Table 5.3. Here we put the group $\Sigma_{\hat{N}}$ of diagram automorphisms (1.3.1) as $\Sigma_{2 n+1}^{\wedge}:=\left\langle\sigma_{0,1}\right\rangle(1.5 .3(4))$ and $\Sigma_{2 n}^{\wedge}:=\left\langle\sigma_{0,1}, \sigma_{n-1, n}\right\rangle(1.5 .5(4))$. 
Example 5.3. The seesaw pair $(\mathrm{b} 0)^{\wedge}$ and $(\mathrm{b} 1)^{\wedge}$ yield the dualities

$$
\begin{aligned}
& \mathbb{B}\left(\mathbb{Z}^{l} \ltimes \mathfrak{g l}(l)^{\wedge} \supset\left(\mathbb{Z}^{l-1} \times \mathfrak{g l}(l-1)^{\wedge}\right) \oplus\left(\mathbb{Z} \times\left(\mathbb{C}^{l}\right)^{\wedge}\right)\right)_{\left(\Lambda_{q}^{p}, 2\right)}^{\left(\Lambda_{p}, 2\right),(r, 2)} \\
& \quad \simeq \mathbb{B}\left(\mathfrak{s l}(2)^{\wedge} \oplus \mathfrak{s l}(2)^{\wedge} \stackrel{\text { diagonal }}{\supset} \mathfrak{s l}(2)^{\wedge}\right)_{\left(p \Lambda_{1}, l-1\right),\left(r \Lambda_{1}, 1\right)}^{\left(q \Lambda_{1}, l\right)} \\
& \simeq \mathbb{B}\left(\mathfrak{s p}(2 l)^{\wedge} \supset \mathfrak{s p}(2 l-2)^{\wedge} \oplus \mathfrak{s p}(2)^{\wedge}\right)_{\hat{\Lambda}_{q}}^{\hat{\Lambda}_{p}, \hat{A}_{r}} \\
& \left(l=2,3, \ldots, 0 \leq p \leq l-1,0 \leq q \leq l, 0 \leq r \leq 1 \text { and } \Lambda_{0}:=0\right) .
\end{aligned}
$$

These branching modules are known to be isomorphic to

$$
\simeq \begin{cases}\mathbb{L}^{\mathscr{V} i}\left(h_{p+1, q+1}(l), c(l)\right) & : \text { if } r \equiv p+q \bmod 2 \\ 0 & : \text { otherwise }\end{cases}
$$

as $\mathscr{V}_{i \imath \text {-modules }}([\mathrm{GKO}],[\mathrm{KW}],[\mathrm{TK} 1,2]) . \quad$ Here $\mathbb{L}^{\mathscr{V}}{ }^{i r}(h, c)$ denotes the irreducible left $\mathscr{V}_{i} r$-module with the highest weight vector $|h, c\rangle(h, c \in \mathbb{C})$ that satisfies

(2) $\left(d(0)+\frac{z}{24}\right)|h, c\rangle=h|h, c\rangle, \quad z|h, c\rangle=c|h, c\rangle, \quad d(m)|h, c\rangle=0 \quad(m>0)$,

(see Notice 0.5 ) and we put

$$
\begin{gathered}
h_{p, q}(l)=h_{l-p, l+1-q}(l):=\frac{\{p(l+2)-q(l+1)\}^{2}-1}{4(l+1)(l+2)}, \\
c(l):=1-\frac{6}{(l+1)(l+2)} .
\end{gathered}
$$

Then it is also known that $\mathrm{L}^{\mathscr{V} i r}(h, c)$ 's for the parameter

$$
(h, c)=\left(h_{p, q}(l), c(l)\right), \quad l \in \mathbb{Z}_{\geq 2}, \quad 1 \leq p<q \leq l+1, \quad p, q \in \mathbb{Z}
$$

exhaust the discrete series unitarizable $\mathscr{V}_{i}$-modules ([GKO]), whose characters are given by the following $\left([\mathrm{FFu}]\right.$; see $1.5 .1(0)$ for $\left.\theta^{\mathbb{Z}}\right)$ :

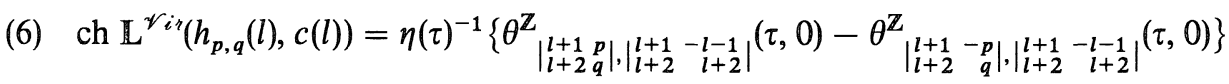
where $\left|\begin{array}{ll}a & b \\ c & d\end{array}\right|:=a d-b c$.

From (1) (2) we can also recover the following result in [TK2].

(7) $\mathbb{B}\left(\mathfrak{s l}(l+1)^{\wedge} \supset \mathfrak{s l}(l)^{\wedge} \oplus\left(\mathbb{Z} \varepsilon_{\perp} \ltimes \mathbb{C} E_{\perp}^{\wedge}\right)\right)_{(Y, 2)}^{(y, 2),(\mu \varepsilon / l(l+1), 2)}$

$$
\begin{gathered}
\simeq \begin{cases}\mathbb{L}^{\mathscr{V} i r}\left(h_{s(y), s(Y)}(l+1), c(l+1)\right) & : \text { if } \mu=l[Y]-(l+1)[y] \\
\mathbb{L}^{\mathscr{V} i r}\left(h_{l-s(y), s(Y)}(l+1), c(l+1)\right) & : \text { if } \mu=l[Y]-(l+1)[y]-l(l+1) \\
0 & : \text { otherwise }\end{cases} \\
\left(Y \in \mathscr{Y}_{l, 2}, y \in \mathscr{Y}_{l-1,2}, 0 \leq \mu \leq 2 l(l+1)\right) .
\end{gathered}
$$

Here we regard $\mathfrak{s l}(l)$ as

$$
\mathfrak{s l}(l)=\left\{\Sigma_{1 \leq i, j \leq l+1} a_{j}^{i} E_{j}^{i} \in \mathfrak{s l}(l+1) \mid a^{l+1}{ }_{j}=0=a_{l+1}^{i}\right\}
$$


and define the Heisenberg system (1.3.3(1)) $\left(\mathbf{Z} \varepsilon_{\perp} \ltimes \mathbf{C} E_{\perp}^{\wedge}\right)=\left(\mathbf{Z} \varepsilon_{\perp} \ltimes\left(\mathbf{C} E_{\perp}\right)_{B}^{\wedge}\right)$ by

$$
\begin{aligned}
\varepsilon_{\perp}:= & \Sigma_{j=1}^{l} \varepsilon_{j}-l \varepsilon_{l+1}, \quad E_{\perp}:=\Sigma_{j=1}^{l} E_{j}^{j}-l E^{l+1}{ }_{l+1} \\
& \left(\text { where } \varepsilon_{j} \in\left(\bigoplus C E^{k}{ }_{k}\right)^{*}, \varepsilon_{j}\left(E^{k}{ }_{k}\right):=\delta_{j}^{k}\right),
\end{aligned}
$$

and $B\left(E_{\perp}, E_{\perp}\right):=l(l+1)$, and put

$$
s(Y):={ }^{t} y_{1}-{ }^{t} y_{2} \in \mathbf{Z}_{\geq 0} \quad \text { for } \quad Y={ }^{t}\left({ }^{t} y_{1},{ }^{t} y_{2}\right) \in \mathscr{Y}_{l, 2} .
$$

Remark 5.3. (i) For a classical affine Lie algebra $X_{l}^{(1)}$ and its invariant subalgebra $\left(X_{l}^{(k)}\right)^{\sigma}$ with respect to a diagram automorphism $\sigma$, see [JM, Table IV] for many examples of $\mathbf{B}\left(X_{l}^{(k)} \supset\left(X_{l}^{(k)}\right)^{\sigma}\right)_{\Lambda}^{\lambda}$ (unfortunately with some "extra factors"), for small level $\Lambda$. In the next paragraph $\S 5.4$ we will deal with such a restriction.

(ii) As for the so-called branching coefficient

$$
\operatorname{ch} \mathbb{B}\left(g^{\wedge} \oplus \mathfrak{g}^{\wedge} \stackrel{\text { diag }}{\supset} \mathfrak{g}^{\wedge}\right)_{\lambda, \mu}^{v}
$$

that arises as the character of the coset Virasoro module associated to the tensor product representation, we can calculate it from the knowledge of string functions. See $[\mathrm{KP} 2, \S 4]$ and [DJKMO, Appendix C] for this point.

\subsection{Affine case (II)}

In the previous section we deal with the dualities that arise as affine analogues of the finite dimensional ones in $\S 5.2$. Now we introduce a new type of seesaw pairs $(\S 5.4 .2$. $(6,7), \S 5.4 .3$. $(1,2)$ ) and the corresponding dualities (Cor. 5.4.2, 5.4.3) that do not have their finite dimensional versions. We will derive them by intertwining between the homogeneous picture representations (our constructions) and the principal picture representations $([\mathrm{F} 2])$.

\subsubsection{The twisted construction of the Virasoro operators}

In the sequel we need the following twisted construction of the Virasoro algebra due to Kac-Peterson [KP2, §2.5].

Let $\mathrm{g}$ be a simple Lie algebra of type $X_{r}, \sigma$ an automorphism of $\mathrm{g}$ of order $N$ which preserves the normalized bilinear form $B$, and

$$
\mathfrak{g}=\bigoplus_{j \in \mathbf{Z} / N \mathbf{Z}} \mathfrak{g}_{j} \quad \text { where } \quad \mathfrak{g}_{j}:=\left\{x \in \mathfrak{g} \mid \sigma(x)=e^{2 \pi \sqrt{-1} j / N} x\right\}
$$

the corresponding gradation. Let $\hat{\mathrm{g}}^{\sigma^{\wedge}}$ be the fixed point subalgebra of $\hat{\mathrm{g}}=\hat{\mathrm{g}}_{B}$ with respect to the automorphism $\sigma^{\wedge}$ defined by

$$
\sigma^{\wedge}(x(n)):=e^{-2 \pi \sqrt{-1} n / N}(\sigma(x))(n) \quad \text { and } \quad \sigma^{\wedge}(c):=c .
$$

Then $\hat{\mathrm{g}}^{\sigma^{\wedge}}$ realizes the Kac-Moody Lie algebra $\mathfrak{g}\left(X_{r}^{(k)}\right)$, where $k$ is the least positive integer that $\sigma^{k}$ is inner ([Kac, $\left.\S 8\right]$; in the following of this paper we only need the case of $k=1$.), and the canonical central element is given by $N c$. Let $g$ be the dual Coxeter number of $\mathfrak{g}\left(X_{r}^{(k)}\right)$. 
Lemma 5.4.1 [KP2, Appendix 3]. Let $\left\{e_{i, r}\right\}$ be a basis of $\mathrm{g}$ that satisfies $e_{i, r} \in \mathrm{g}_{r}$ and $B\left(e_{i, r}, e_{j, s}\right)=\delta_{i, j} \delta_{r+s, 0}$. Then

$$
\begin{aligned}
D(k)=D^{\hat{\mathrm{g}}^{\wedge}}(k):= & \frac{1}{2(g+l)} \sum_{r \in \mathbb{Z}} \sum_{i=1}^{\operatorname{dim} \mathrm{g}_{r}}{ }_{0} e_{i, r}(r) e_{i,-r}(N k-r)_{\circ}^{\circ} \\
& -\delta_{k, 0} \frac{l}{g+l}\left(\frac{\operatorname{dim} \mathrm{g}}{24}-\Sigma_{j=1}^{N} j(N-j)\left(\operatorname{dim} \mathfrak{g}_{j}\right) / 4 N^{2}\right)
\end{aligned}
$$

defines a representation of $\mathscr{V}_{i r}$ on any $\hat{\mathrm{g}}^{\sigma^{\wedge}}$-module that satisfies $1.2 .2(1)$ and (2) (see also 1.2.2(4) for $\left.\stackrel{\circ}{\circ}{ }_{\circ}^{\circ}\right)$ : on such a module we have

$$
[D(m), x(n)]=-\frac{n}{N} x(N m+n)
$$

for $x(m) \in \hat{\mathrm{g}}^{\sigma^{\wedge}}$, and

$$
[D(m), D(n)]=(m-n) D(m+n)+\delta_{m+n, 0} \frac{m^{3}}{12} \frac{l \operatorname{dim} g}{l+g} i d .
$$

5.4.2. The restriction $\left(\mathfrak{s l}(m r)^{\wedge}\right)^{\left\langle\sigma_{c y c}{ }^{r}\right\rangle} \subset \mathfrak{s l}(m r)^{\wedge}$ and îts counterpart $\mathrm{gl}(l) \otimes \mathbb{C}\left[s, s^{-1}\right] \oplus \mathbb{C} c \supset \operatorname{gl}(l) \otimes \mathbb{C}\left[s^{m}, s^{-m}\right] \oplus \mathbb{C} c$

We go back to dual pairs. Fix $l, m, r \in \mathbb{Z}_{>0}$. This time we remark that there is an algebra isomorphism

$$
C\left(W_{2 l r}^{\mathrm{Z}+1 / 2}\right) \simeq C\left(W_{2 l m r}^{\mathrm{Z}+1 / 2}\right)
$$

induced by the isometry given by the assignation

$$
\begin{gathered}
W_{2 l r}^{\mathbf{Z}+1 / 2} \ni \psi^{j, p}\left(\alpha+m \mu-\frac{1}{2}\right) \leftrightarrow \psi^{j, \alpha, p}\left(\mu+\frac{1}{2}\right) \in W_{2 l m r}^{\mathbb{Z}+1 / 2} \\
\psi_{j, p}\left(-\alpha+m \mu+\frac{1}{2}\right) \leftrightarrow \psi_{j, \alpha, p}\left(\mu-\frac{1}{2}\right)
\end{gathered}
$$

for $1 \leq j \leq l, 1 \leq \alpha \leq m, 1 \leq p \leq r, \mu \in \mathbb{Z}$. Here $\psi^{a}(v)$ and $\psi_{a}(v)(a=(j, p)$ or $(j, \alpha, p)$, $v \in \mathbb{Z}+1 / 2$ ) are the basis of $W_{2 n}^{\mathbb{Z}+1 / 2}$ such that

$$
\left\{\psi^{a}(\mu), \psi_{b}(v)\right\}=\delta_{b}^{a} \delta_{\mu+v, 0} \quad \text { and } \quad\left\{\psi^{a}(\mu), \psi^{b}(v)\right\}=0=\left\{\psi_{a}(\mu), \psi_{b}(v)\right\} .
$$

The isometry (1) preserves the isotropic decompositions $(2.4(2))$ and hence induces the following Clifford algebra module isomorphisms

$$
\bigwedge\left(W_{2 l r}^{\mathrm{Z}+1 / 2,-}\right) \underset{\rightrightarrows}{\rightrightarrows} \bigwedge\left(W_{2 l m r}^{\mathbf{Z}+1 / 2,-}\right)
$$

and

$$
\omega: \text { End } \bigwedge\left(W_{2 l r}^{\mathbf{Z}+1 / 2,-}\right) \underset{\Im}{\cong} \text { End } \bigwedge\left(W_{2 l m r}^{\mathbb{Z}+1 / 2,-}\right) .
$$

Theorem 4.2 says that in the left hand side of (5) there is a dual pair

$$
\begin{array}{cc}
\left(\mathbb{Z}^{l} \ltimes \operatorname{gl}(l)_{s}^{\wedge},\right. & \left.s \mathrm{sl}(r)_{s}^{\wedge}\right), \\
\text { level } r \quad \text { level } l
\end{array}
$$


while in the right hand side so is a pair

$$
\begin{array}{cc}
\left(Z^{l} \ltimes \mathfrak{g l}(l)_{t}^{\wedge}, \mathfrak{s l}(m r)_{t}^{\wedge}\right) \\
\text { level } m r \quad \text { level } l
\end{array}
$$

Here and after we write $\operatorname{gl}(l)_{s}^{\wedge}=\operatorname{gl}(l) \otimes \mathbf{C}\left[s, s^{-1}\right] \oplus \mathbf{C} c$, etc in order to distinguish the mutually isomorphic algebras according to the spaces that they act on. Moreover we observe that

Proposition 5.4.2. (i) Under the isomorphism (5), the following inclusion relations (8) and (9) hold.

$$
\begin{aligned}
& \mathbb{Z}^{l} \ltimes \mathfrak{g l}(l)_{s}^{\wedge} \supset \mathbb{Z}^{l} \ltimes \operatorname{gl}(l)_{t}^{\wedge} \\
& \text { (level } r \text { ) (level } \mathrm{mr} \text { ) } \\
& \mathfrak{s l}(r)_{s}^{\wedge} \subset \mathfrak{s l}(m r)_{t}^{\wedge} \\
& \text { (level l) (level l) }
\end{aligned}
$$

i.e. the two dual pairs (6) and (7) form a seesaw pair.

(ii) More precisely, (8) and (9) are respectively given by the following:

$$
\begin{aligned}
& \mathbb{Z}^{l} \ltimes \mathfrak{g l}(l)_{s}^{\wedge} \ni\left(m \gamma, x \otimes s^{m v}\right) \stackrel{\omega^{-1}}{\hookleftarrow}\left(\gamma, x \otimes t^{\nu}\right) \in \mathbb{Z}^{l} \ltimes \mathfrak{g l}(l)_{t}^{\wedge} \\
& m c \leftrightarrow c, \\
& \left.\omega\right|_{\mathfrak{s l}(r)_{s}^{\wedge}}: \quad \mathfrak{s l}(r)_{s}^{\wedge} \quad \stackrel{\omega}{\hookrightarrow} \quad \quad \mathfrak{s l}(m r)_{t}^{\wedge} \\
& \Psi \\
& \left\{\begin{array}{ccc}
E_{q}^{p} \otimes s^{\alpha+m \nu} & \mapsto\left(\sum_{\beta=1}^{\alpha} E_{m+\beta-\alpha}^{\beta} \otimes E_{q}^{p}\right) \otimes t^{\nu+1} \\
& +\left(\sum_{\beta=\alpha+1}^{m} E_{\beta-\alpha}^{\beta} \otimes E_{q}^{p}\right) \otimes t^{\nu} \\
c & \mapsto & c
\end{array}\right. \\
& \left(1 \leq p, q \leq r, 1 \leq \alpha \leq m, v \in \mathbf{Z}, \gamma \in \mathbf{Z}^{l}\right) .
\end{aligned}
$$

Here we identify ${E^{\alpha}}_{\beta} \otimes E^{p}{ }_{q} \in \operatorname{Mat}(m, m) \otimes \operatorname{Mat}(r, r)$ with $E_{\beta+m q}^{\alpha+m p} \in \operatorname{Mat}(m r, m r)(1 \leq \alpha$, $\beta \leq r ; 1 \leq p, q \leq m)$.

(iii) In particular, the image of the latter coincides with the fixed point set $\left(\mathfrak{s l}(m r)_{t}^{\wedge}\right)^{\left\langle\sigma_{c y c}{ }^{r}\right\rangle} \subset \mathfrak{s l}(m r)_{t}^{\wedge}$ of the order $m$ cyclic automorphism $\left(\sigma_{c y c}\right)^{r}$, where $\sigma_{c y c}$ denotes the order $\mathrm{mr}$ cyclic diagram automorphism (1.5.2(4)) of $\mathfrak{s l}(m r)_{t}^{\wedge}$.

Proof. On $\bigwedge\left(W_{2 l r}^{\mathrm{Z}+1 / 2,-}\right) \simeq \bigwedge\left(W_{2 l m r}^{\mathrm{Z}+1 / 2,-}\right)$, we have

$$
\begin{aligned}
& E^{p}{ }_{q} \otimes s^{\alpha+m n}= \sum_{j=1}^{l} \Sigma_{\mu \in \mathbf{Z}}: \psi^{j, p}\left(\mu-\frac{1}{2}\right) \psi_{j, q}\left(\alpha+m n-\mu+\frac{1}{2}\right): \\
&= \sum_{j=1}^{l} \Sigma_{v \in \mathbf{Z}} \Sigma_{\beta=1}^{m}: \psi^{j, p}\left(\beta+m v-\frac{1}{2}\right) \psi_{j, q}\left(\alpha+m n-\beta-m v+\frac{1}{2}\right): \\
& \stackrel{\omega}{\mapsto} \sum_{j=1}^{l} \Sigma_{v \in \mathbf{Z}} \Sigma_{\beta=\alpha+1}^{m}: \psi^{j, \beta, p}\left(v+\frac{1}{2}\right) \psi_{j, \beta-\alpha, q}\left(m(n-v)-\frac{1}{2}\right): \\
& \quad+\sum_{j=1}^{l} \Sigma_{v \in \mathbf{Z}} \Sigma_{\beta=1}^{\alpha}: \psi^{j, \beta, p}\left(v+\frac{1}{2}\right) \psi_{j, \beta-\alpha+m, q}\left(m(n-v+1)-\frac{1}{2}\right):
\end{aligned}
$$

since (2) preserves the normal products. Together with verification of the corre- 
spondence of Chevalley generators, this proves the claim (8). The statement (9) is obtained similarly.

Remark 5.4.2. In case of $l=r=1$, the homogeneous picture representation of $\mathrm{gl}(m)^{\wedge}$ on $\bigwedge\left(W_{2 m}^{\mathbb{Z}+1 / 2,-}\right)$ induces that on $\bigwedge\left(W_{2}^{\mathbb{Z}+1 / 2,-}\right)$ through the isomorphism $(1) \sim(5)$, which is nothing but the so-called principal picture representation ([Kac, $\S \S 14.7,14.13])$.

To obtain the coset $\mathscr{V}_{i}$-operators associated to the inclusion $\left(9^{\prime}\right)$, we shall clarify the graded structure of its image. Let $\sigma$ be the inner automorphism of $\mathfrak{s l}(\mathrm{mr})$ defined by

$$
\sigma(x \otimes y):=\operatorname{ExE}^{-1} \otimes y \quad(x \in \mathfrak{g l}(m), y \in \mathfrak{g l}(r)),
$$

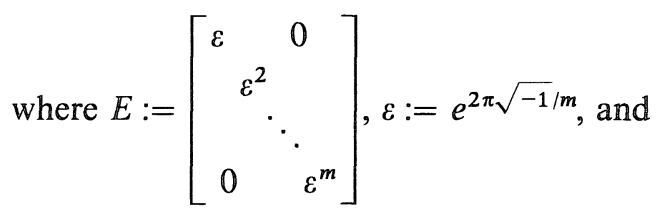

$$
\begin{aligned}
\left(\mathfrak{s l}(m r)_{u}^{\wedge}\right)^{\sigma^{\wedge}} & :=\left(\bigoplus_{\substack{\alpha-\beta \equiv n \\
\bmod m}} \mathbb{C} E_{\beta}^{\alpha} \otimes E^{p}{ }_{q} \otimes u^{n}\right) \oplus \mathbb{C} c \\
& \subset \mathfrak{s l}(m r) \otimes \mathbb{C}\left[u, u^{-1}\right] \oplus \mathbb{C} c
\end{aligned}
$$

the associated affine Lie algebra ( $\$ 5.4 .1)$. We have an isomorphism

$$
\begin{aligned}
\varphi: \mathfrak{s l}(m r)_{t}^{\wedge} \ni E_{\beta}^{\alpha} \otimes E_{q}^{p} \otimes t^{n} & \stackrel{\cong}{\leftrightarrows} \mathbb{E}_{\beta}^{\alpha} \otimes \mathbb{E}_{q}^{p} \otimes u^{m n+\alpha-\beta} \in\left(\mathfrak{s l}(m r)_{u}^{\wedge}\right)^{\sigma^{\wedge}} \\
c & \mapsto m c
\end{aligned}
$$

where $1 \leq \alpha, \beta \leq m, \quad 1 \leq p, q \leq r$ and $n \in \mathbb{Z} . \quad \mathbb{P u t} \mathbb{P}:=\left[\begin{array}{ccc}0 & & 1 \\ 1 & \ddots & 0 \\ 0 & \ddots & \ddots \\ & 1 & 0\end{array}\right] \in \mathfrak{s l}(m)$. Then from Proposition 5.4.2 it is easy to see

Lemma 5.4.2. We have

$$
\varphi \circ \omega\left(E_{q}^{p} \otimes s^{n}\right)=\mathbb{P}^{n} \otimes \mathbb{E}_{q}^{p} \otimes u^{n}, \quad \varphi \circ \omega(c)=m c
$$

and

$$
\varphi \circ \omega\left(\mathfrak{s l}(r)_{s}^{\wedge}\right)=\left(\mathfrak{s l}(m r)_{u}^{\wedge}\right)^{\left\langle\sigma^{\wedge}, \operatorname{Ad}(P) \otimes 1\right\rangle} \subset\left(\mathfrak{s l}(m r)_{u}^{\wedge}\right)^{\sigma^{\wedge}},
$$

where $\operatorname{Ad}(P) \otimes 1$ denotes the automorphism of $\left(\mathfrak{s l}(m r)_{u}^{\wedge}\right)^{\sigma^{\wedge}}$ given by

$$
\operatorname{Ad}(P) \otimes 1: x \otimes y \otimes u^{n} \mapsto \mathbb{P} x \mathbb{P}^{-1} \otimes y \otimes u^{n}, \quad c \mapsto c,
$$

and $\left(\mathfrak{s l}(m r)_{u}^{\wedge}\right)^{\left\langle\sigma^{\wedge}, \operatorname{Ad}(P) \otimes 1\right\rangle}$ stands for the fixed point subalgebra of $\mathfrak{s l}(m r)_{u}^{\wedge}$ concerning with $\sigma^{\wedge}$ and $\operatorname{Ad}(P) \otimes 1$.

Thanks to this lemma we can introduce a $\mathscr{V}_{i} \iota$-module structure into the branching module

$$
\mathbb{B}\left(\mathfrak{s l}(m r)_{t}^{\wedge} \supset\left(\mathfrak{s l}(m r)_{t}^{\wedge}\right)^{\left\langle\sigma_{c y c}^{m}\right\rangle}\right)_{(Y ; l)}^{(y ; l)} \simeq \mathbb{B}\left(\varphi^{-1}\left(\left(\mathfrak{s l}(m r)_{u}^{\wedge}\right)^{\sigma^{\wedge}}\right) \supset \omega\left(\mathfrak{s l}(r)_{s}^{\wedge}\right)\right)_{(Y ; l)}^{(y ; l)}
$$


by the difference of the twisted Virasoro operators

$$
d(k) \mapsto D^{(15)}(k):=\varphi^{-1}\left(D^{\left(\mathfrak{s I}(m r)_{\hat{u}}\right)^{\kappa \wedge}}(k)\right)-\omega\left(D^{\mathfrak{s I}(r)_{s}^{\hat{s}}}(k)\right),
$$

and

$$
z \mapsto\left(\frac{l\left(m^{2} r^{2}-1\right)}{l+m r}-\frac{l\left(r^{2}-1\right)}{l+r}\right) i d .
$$

(In (15), we extend the isomorphism $\varphi^{-1}$ (12) of Lie algebras to that of (formally completed) enveloping algebras.) Note that the branching module

$$
\mathbb{B}\left(\mathbb{Z}^{l} \ltimes \operatorname{gl}(l)_{s}^{\wedge} \supset \omega^{-1}\left(\mathbb{Z}^{l} \ltimes \operatorname{gl}(l)_{t}^{\wedge}\right)\right)_{(y,[y] ; r)}^{(X,[Y] ; m r)} \simeq \mathbb{B}\left(\mathbb{Z}^{l} \ltimes \operatorname{gl}(l)_{s}^{\wedge} \supset \mathbb{Z}^{l} \ltimes \operatorname{gl}(l)_{s^{m}}^{\wedge}\right)_{(y,[y] ; r)}^{(Y,[Y] ; m r)}
$$

associated to $\left(8^{\prime}\right)$ also admits the $\mathscr{V}_{i}$-action defined by

$$
d(k) \mapsto D^{(16)}(k):=\frac{1}{m} D^{g^{\mathrm{I}(l) \hat{s}}}(m k)-\omega^{-1}\left(D^{\mathrm{g}^{\mathrm{I}(l) \hat{t}}}(k)\right)
$$

and

$$
z \mapsto\left(m\left(1+\frac{r \cdot\left(l^{2}-1\right)}{l+r}\right)-\frac{m r\left(l^{2}-1\right)}{m r+l}\right) i d .
$$

Now the seesaw pair $(8,9)$ yields the following duality (i).

Corollary 5.4.2.(i) For $Y \in \mathscr{Y}_{l, m r-1}$ and $y \in \mathscr{Y}_{l, r-1}$, we have the isomorphism

$$
\begin{aligned}
& \mathbb{B}\left(\mathbb{Z}^{l} \ltimes\left(\mathfrak{g l}(l) \otimes \mathbb{C}\left[s, s^{-1}\right] \oplus \mathbb{C} c\right) \supset m \mathbb{Z}^{l} \ltimes\left(\mathfrak{g l}(l) \otimes \mathbb{C}\left[s^{m}, s^{-m}\right] \oplus \mathbb{C} c\right)\right)_{(y,[y] ; r)}^{(Y,[Y] ; m r)} \\
& \quad \simeq \mathbb{B}\left(\mathfrak{s l}(m r)^{\wedge} \supset\left(\mathfrak{s l}(m r)^{\wedge}\right)^{\left\langle\sigma_{c y c}{ }^{r}\right\rangle}\right)_{(t ; y ; l)}^{(t y ; l)}
\end{aligned}
$$

of the coset $\mathscr{V}$ ir-modules defined by (16) and (15) respectively, where we parametrize $\left(\mathfrak{s l}(m r)^{\wedge}\right)^{\left\langle\sigma_{c y c}{ }^{r}\right\rangle}$-modules by ${ }^{t} y \in \mathscr{Y}_{r-1, l}$ according to the isomorphism (Prop. 5.4.2(iii))

$$
\mathfrak{s l}(r)^{\wedge} \stackrel{\left(9^{\prime}\right)}{\rightarrow}\left(\mathfrak{s l}(m r)^{\wedge}\right)^{\left\langle\sigma_{c y c} r\right\rangle} .
$$

(iii) The matrix of the branching modules

$$
\mathbb{B}\left(\mathfrak{s l}(m r)^{\wedge} \supset\left(\mathbb{1}_{m} \otimes \mathfrak{s l}(r)\right)^{\wedge}\right):=\left(\mathbb{B}\left(\mathfrak{s l}(m r)^{\wedge} \supset\left(\mathbb{1}_{m} \otimes \mathfrak{s l}(r)\right)^{\wedge}\right)_{(Y ; l)}^{(y ; m l)}\right)_{Y \in \mathscr{Y}_{m r-1, l}, y \in \mathscr{Y}_{r-1, m l}}
$$

associated to the inclusion in Table 5.2.a3 factors into the form

$$
\begin{aligned}
& \mathbb{B}\left(\mathfrak{s l}(m r)^{\wedge} \supset\left(\mathbb{1}_{m} \otimes \mathfrak{s l}(r)\right)^{\wedge}\right) \\
& =\mathbb{B}\left(\mathbb{Z}^{l} \ltimes\left(\mathfrak{s l}(l) \otimes \mathbb{C}\left[t, t^{-1}\right] \oplus \mathbb{C} c\right) \supset m \mathbb{Z}^{l} \ltimes\left(\operatorname{gl}(l) \otimes \mathbb{C}\left[t^{m}, t^{-m}\right] \oplus \mathbb{C} c\right)\right) \\
& \left.\otimes \mathbb{B}\left(\mathfrak{s l}(r) \otimes \mathbb{C}\left[t, t^{-1}\right] \oplus \mathbb{C} c \supset \mathfrak{s l}(r) \otimes \mathbb{C}\left[t^{m}, t^{-m}\right] \oplus \mathbb{C} c\right)\right) .
\end{aligned}
$$

Proof. (i) Our task is to prove the equality $D^{(15)}(k)=D^{(16)}(k)$ of the coset $\mathscr{V}_{i} i_{-}$ operators for any $k \in \mathbb{Z}$, which is valid similarly as in Corollary 5.3a if we can show the identity

$$
\omega\left(\frac{1}{m} D^{\mathrm{gl}(l) \hat{s}}(m k)+\frac{1}{m} D^{\mathrm{sI}(r) \hat{s}}(m k)\right)-\left(D^{\mathrm{gl}(l) \hat{t}}(k)+\varphi^{-1}\left(D^{\left(\mathfrak{s l}(m r) \hat{u}^{\hat{u}}\right)^{\sigma \wedge}}(k)\right)\right)=0 .
$$

To prove (17), we have only to show that the left hand side above commutes with 
$\mathfrak{g l}(l)_{t}^{\wedge} \oplus \mathfrak{s l}(m r)_{t}^{\wedge}$, because Proposition 1.2(ii) $\rightarrow($ iii) works then since

$$
m\left(1+\frac{r\left(l^{2}-1\right)}{l+r}+\frac{l\left(r^{2}-1\right)}{r+l}\right)=l m r=1+\frac{l\left(m^{2} r^{2}-1\right)}{l+m r}+\frac{m r\left(l^{2}-1\right)}{m r+l}
$$

holds. Using Proposition 4.1(i) we have

$$
\begin{aligned}
& \frac{1}{m}\left(D^{\mathrm{gl}(l) \hat{s}}(m k)+D^{\mathfrak{s l}(r) \hat{s}}(m k)+\frac{l r}{24} \delta_{k, 0}\right) \\
& \quad=\frac{1}{m}\left(D^{C l i f_{2 l r}}(m k)+\frac{l r}{24} \delta_{k, 0}\right) \\
& \quad=\sum_{j=1}^{l} \sum_{p=1}^{r} \sum_{\mu \in \mathbb{Z}+1 / 2} \frac{m k-2 \mu}{2 m}: \psi^{j, p}(\mu) \psi_{j, p}(m k-\mu): \\
& \quad=\sum_{j=1}^{l} \sum_{p=1}^{r} \sum_{\alpha=1}^{m} \sum_{v \in \mathbb{Z}} \frac{m k+2(\alpha+m v-1 / 2)}{2 m}: \psi^{j, p}\left(\alpha+m v-\frac{1}{2}\right) \psi_{j, p}\left(m k-\alpha-m v+\frac{1}{2}\right): \\
& \stackrel{\omega}{\mapsto} \sum_{j=1}^{l} \sum_{p=1}^{r} \sum_{\alpha=1}^{m} \sum_{v \in \mathbb{Z}}\left(\frac{k+2 v}{2}+\frac{\alpha-1 / 2}{m}\right): \psi^{j, \alpha, p}\left(v+\frac{1}{2}\right) \psi_{j, \alpha, p}\left(k-v-\frac{1}{2}\right): \\
& \quad=D^{C l i f_{2 l m r}}(k)+\frac{l m r}{24} \delta_{k, 0}+\sum_{\alpha=1}^{m} \frac{\alpha-1 / 2}{m}\left(\mathbb{1}_{l} \otimes E_{\alpha}^{\alpha} \otimes \mathbb{1}_{r}\right)(k) .
\end{aligned}
$$

This expression enables us to compute the commutator in problem. Together with Lemma 5.4 .1 and (11) we know that the left hand side of (17) actually commutes with $\mathrm{gl}(l)_{t}^{\wedge} \oplus \mathfrak{s l}(m r)_{t}^{\wedge}$, proving the statement.

(ii) Discussions in this paragraph (5.4.2) can be summarized as the following diagram.

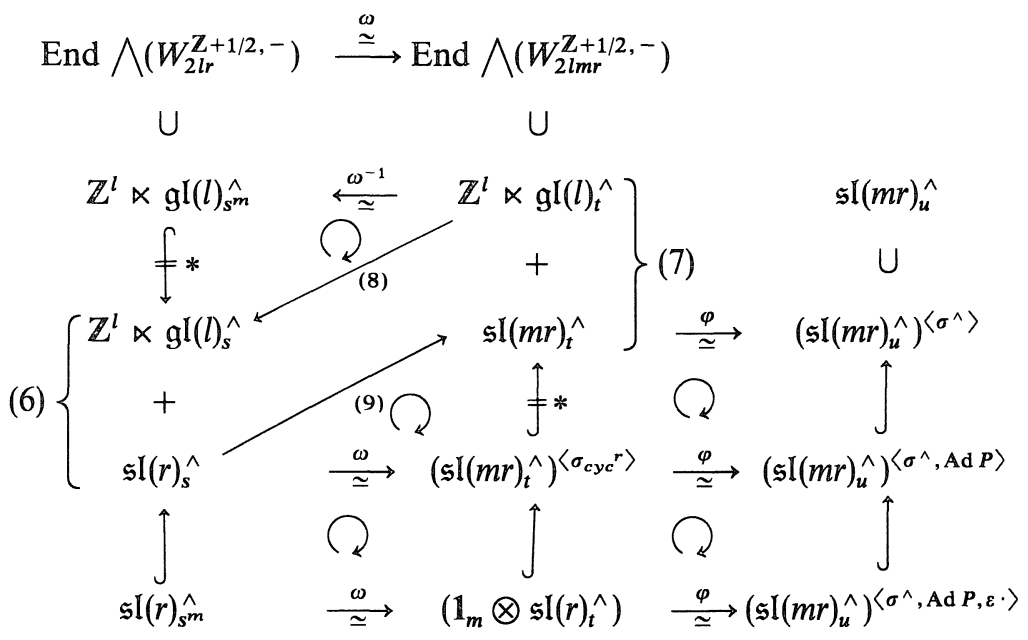

$\left((*):{ }^{*}="\right.$ means the isomorphism in Cor. 5.4.2(i).)

Here $\mathfrak{s l}(r)_{s}^{\wedge}=\mathfrak{s l}(r) \otimes \mathbb{C}\left[s, s^{-1}\right] \oplus \mathbb{C} c$, etc. and $\mathrm{Ad} P$ and $\varepsilon \cdot$ denote the automorphisms defined by (11) and by $x \otimes s^{k} \mapsto x \otimes(\varepsilon s)^{k}, \varepsilon:=e^{2 \pi i / m}$, respectively. Now using (i), the assertion follows as 


$$
\begin{aligned}
& \mathbf{B}\left(\mathfrak{s l}(m r)_{t}^{\wedge} \supset\left(\mathbb{1}_{m} \otimes \mathfrak{s l}(r)\right)_{t}^{\wedge}\right) \\
& \quad=\mathbf{B}\left(\mathfrak{s l}(m r)_{t}^{\wedge} \supset\left(\mathfrak{s l}(m r)_{t}^{\wedge}\right)^{\left\langle\sigma_{c y c} r^{r}\right\rangle}\right) \otimes \mathbf{B}\left(\left(\mathfrak{s l}(m r)_{t}^{\wedge}\right)^{\left\langle\sigma_{c y c} c^{r\rangle} \supset\left(\mathbb{1}_{m} \otimes \mathfrak{s l}(r)\right)_{t}^{\wedge}\right)}\right. \\
& \quad=\mathbf{B}\left(\mathbb{Z}^{l} \ltimes \mathfrak{g l}(l)_{u}^{\wedge} \supset m \mathbb{Z}^{l} \ltimes \mathfrak{g l}(l)_{u^{m}}^{\wedge}\right) \otimes \mathbb{B}\left(\mathfrak{s l}(r)_{u}^{\wedge} \supset \mathfrak{s l}(r)_{u^{m}}^{\wedge}\right) .
\end{aligned}
$$

5.4.3. The restriction $\mathfrak{s p}(2 l) \otimes \mathbf{C}\left[t, t^{-1}\right] \oplus \mathbf{C} c \supset \mathfrak{s p}(2 l) \otimes \mathbf{C}\left[t^{m}, t^{-m}\right] \oplus \mathbb{C} c$ and the diagram automorphism of $C^{(1)}{ }_{2}$.

We can apply the method in the previous paragraph (5.4.2.) to other types of classical affine Lie algebras. For example, here we will deduce the type $C_{l}^{(1)}$ version for Cor. 5.4.2.

We start with the isometry $W_{4 l r}^{\mathbf{Z}+1 / 2} \simeq W_{4 l m r}^{\mathbb{Z}+1 / 2}$ which is similary obtained as in 5.4.2(2). It induces the algebra isomorphisms $C\left(W_{4 l r}^{\mathbf{Z}+1 / 2}\right) \simeq C\left(W_{4 l m r}^{\mathrm{Z}+1 / 2}\right)$ and

$$
\text { End } \bigwedge\left(W_{4 l r}^{\mathrm{Z}+1 / 2,-}\right) \simeq \text { End } \bigwedge\left(W_{4 l m r}^{\mathrm{Z}+1 / 2,-}\right) \text {. }
$$

Theorem 4.2 yields the following seesaw pair of affine Lie algebras.

$$
\begin{array}{cc}
\mathfrak{s p}(2 l)^{\wedge} & \supset \mathfrak{s p}(2 l)^{\wedge} \\
(\text { level } r) & (\text { level } m r) \\
+ & + \\
\mathfrak{s p}(2 r)^{\wedge} & \subset \mathfrak{s p}(2 m r)^{\wedge} \\
(\text { level } l) & (\text { level } l)
\end{array} .
$$

Then the corresponding duality is the following

Corollary 5.4.3 [Has, §3]. (i) As coset $\mathscr{V}$ ir-modules, we have

$$
\begin{gathered}
\mathbb{B}\left(\mathfrak{s p}(2 l) \otimes \mathbb{C}\left[t, t^{-1}\right] \oplus \mathbb{C} c \supset \mathfrak{s p}(2 l) \otimes \mathbb{C}\left[t^{m}, t^{-m}\right] \oplus \mathbb{C} \cdot m c\right)_{(t y, r)}^{(t Y, m r)} \\
\simeq \mathbb{B}\left(\mathfrak{s p}(2 m r)^{\wedge} \stackrel{b}{\leftrightarrows} \mathfrak{s p}(2 r)^{\wedge}\right)_{(Y, l)}^{(y, l)} \quad\left(Y \in \mathscr{Y}_{m r, l}, y \in \mathscr{Y}_{r, l}\right),
\end{gathered}
$$

where $l$ is the Lie algebra homomorphism defined as follows.

$$
\begin{aligned}
\mathfrak{s p}(2 r)^{\wedge} \ni C_{q}^{p}(\alpha+k m) & \mapsto \sum_{\beta=1}^{l} C_{q, m+\beta-\alpha}^{p, \beta}(k+1)+\sum_{\beta=\alpha+1}^{m} C_{q, \beta-\alpha}^{p, \beta}(k) \quad \in \mathfrak{s p}(2 m r)^{\wedge} \\
C^{p ; q}(\alpha+k m) & \mapsto \sum_{\beta=1}^{\alpha} C^{p, \beta ; q, \alpha-\beta+1}(k+1)+\sum_{\beta=\alpha+1}^{m} C^{p, \beta ; q, m+\alpha-\beta+1}(k) \\
C_{p ; q}(\alpha+k m) & \mapsto \sum_{\beta=1}^{m-\alpha} C_{p, \beta ; q \cdot-\alpha-\beta+1}(k)+\sum_{\beta=m+1-\alpha}^{m} C_{p, \beta ; q, 2 m-\alpha-\beta+1}(k+1) \\
c & \mapsto c \quad(1 \leq \alpha \leq m, k \in \mathbb{Z} \text { and } 1 \leq p, q \leq r .)
\end{aligned}
$$

Here we put

$$
C_{q, \beta}^{p, \alpha}:=C_{q+m \beta}^{p+m \alpha}, \quad C_{p, \alpha ; q, \beta}:=C_{p+m \alpha, q+m \beta} \quad \text { and } \quad C^{p, \alpha ; q, \beta}:=C^{p+m \alpha, q+m \beta} \in \mathfrak{s p}(2 m r) .
$$

(ii) When $m=2, \quad l\left(\mathfrak{s p}(2 r)^{\wedge}\right)=\left\{x \in \mathfrak{s p}(4 r)^{\wedge} \mid \sigma_{w}(x)=x\right\}$, where $\sigma_{w}$ is the order 2 diagram automorphism of $\mathfrak{s p}(4 r)^{\wedge}=\mathfrak{g}\left(C_{2 r}{ }^{(1)}\right)(\S 1.5 .4)$.

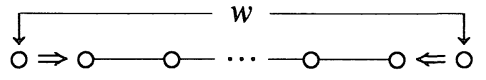


Proof. Together with Theorem 4.2 (Case $\hat{\mathrm{C}} \hat{\mathrm{C}}, h=1 / 2$ ) and Proposition 5.2, (i) immediately follows from the explicit description of inclusions (2) and (3). (The linear map $l$ is just the inclusion (2) and coincides with the restriction of the homomorphism $\left(5.4 .2\left(9^{\prime}\right)\right)$ to $\mathfrak{s p}(2 r)^{\wedge}$.) The proof of (ii) is given by the straightforward verification of correspondence of the Chevalley generators.

From (ii), we can recognize Jimbo-Miwa's result [JM, Proposition 3.11, Case (5)] as a special case $(m=2)$ of (i).

\section{§6. An Application to the Dualing of Modular Transformation Rules of Characters}

6.0. In 6.1 we summarize the $S$-transformation rules of spin module characters. They are applied to deduce some "duality" on the transformation rules of affine Lie algebra characters in 6.2. The author owes the contents of this section to Professor Tsuchiya.

\subsection{S-transformation rulles of spin module characters}

Recall the definition of $\operatorname{ch}_{N}^{+}, \mathbb{Z}+h$ in $\S 2.4$ :

$$
\operatorname{ch}_{N}^{ \pm}, \mathbb{Z}+h:=\operatorname{ch}_{\bigwedge^{\operatorname{even}\left(W_{N}^{Z+h,-}\right)}} \pm \operatorname{ch}_{\left.\bigwedge^{\text {odd }\left(W_{N}^{Z}+h .^{-}\right)}\right)}
$$

and the definition of the modular transformation for the function on $\tilde{\mathfrak{h}}=\mathfrak{h}^{\mathfrak{o}(N)} \oplus \mathbb{C} \mathfrak{c} \oplus$ $\mathbb{C} d: \quad \chi \circ A(h):=\chi(A(h))$,

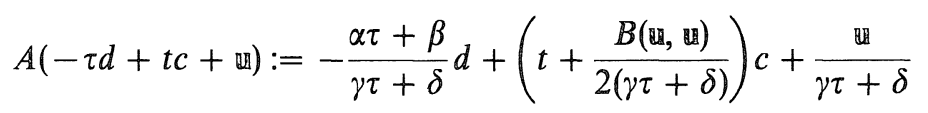

$\left(A=\left[\begin{array}{ll}\alpha & \beta \\ \gamma & \delta\end{array}\right] \in S L(2, \mathbb{Z}), \tau, t \in \mathbb{C}\right.$, a $\left.\in \mathfrak{h}\right)$. Then the well-known transformation rules for $\theta, \hat{\theta}$ and $\eta(\S 1.5 .1)$ yields

Proposition 6.1. Under the transformation by $\mathbb{S}:=\left[\begin{array}{rr}0 & -1 \\ 1 & 0\end{array}\right]$, the character $\operatorname{ch}_{\bar{N}}^{ \pm}, \mathbb{Z}+h$ ( $h=0,1 / 2 ; N=1,2)$ of the spin modules transforms as in Table 6.1.

\begin{tabular}{|c|c|c|c|c|}
\hline case & $h$ & \pm & $\mathrm{ch}_{1}^{ \pm}, \mathrm{Z}+h$ & $\mathrm{ch}_{1}^{ \pm}, \mathrm{Z}+h \circ S$ \\
\hline (a) & \multirow{2}{*}{$\frac{1}{2}$} & + & $\frac{\eta(\tau)^{2}}{\eta(2 \tau) \eta(\tau / 2)}$ & $\times 1$ \\
\hline (b) & & - & $\frac{\eta(\tau / 2)}{\eta(\tau)}$ & \multirow{2}{*}{$x_{\times \sqrt{2}}^{\times 1 / \sqrt{2}}$} \\
\hline (c) & \multirow{2}{*}{0} & + & $\frac{\eta(2 \tau)}{\eta(\tau)}$ & \\
\hline (d) & & - & $\eta(\tau)$ & $\times \sqrt{-i \tau}$ \\
\hline
\end{tabular}

Table 6.1.

\begin{tabular}{|c|c|c|c|c|}
\hline case & $h$ & \pm & $\eta(\tau) \operatorname{ch}_{2}^{ \pm}, \mathbf{Z}+h$ & $\operatorname{ch}_{2}^{ \pm}, \mathrm{Z}+h \circ S$ \\
\hline (e) & \multirow{2}{*}{$\frac{1}{2}$} & + & $\hat{\theta}\left(\tau, u+\frac{1}{2}\right)$ & $\times 1$ \\
\hline (f) & & - & $\hat{\theta}(\tau, u)$ & \\
\hline (g) & \multirow{2}{*}{0} & + & $-i \theta\left(\tau, u+\frac{1}{2}\right)$ & \\
\hline (h) & & - & $-i \theta(\tau, u)$ & $\times(-i)$ \\
\hline
\end{tabular}


Namely, case (c) of Table 6.1 tells that

$$
\operatorname{ch}_{1}^{+, \mathbb{Z}}\left(-\frac{1}{\tau} D_{0}\right)=\sqrt{2^{-1}} \times \operatorname{ch}_{1}^{-,}, \mathbf{Z}+1 / 2\left(\tau D_{0}\right),
$$

and case $(h)$ tells

$$
\left.\operatorname{ch}_{2}^{+,} \mathbf{z}\left(-\frac{1}{\tau} D_{0}+\frac{u}{\tau} D_{1}^{1}+\frac{u^{2}}{\tau} c\right)=(-i) \times \operatorname{ch}_{2}^{+,}, \mathbf{Z}_{\left(\tau D_{0}\right.}+u D^{1}{ }_{1}\right),
$$

where $D_{0}:=D^{\text {Clif }}(0)(2.4(15))$ and $D_{1}^{1}=\left[\begin{array}{rr}1 & 0 \\ 0 & -1\end{array}\right] \in \mathfrak{h}^{\mathfrak{o}(2)}$.

\subsection{A duality of transformation rules of affine Lie algebra characters}

In Theorem 4.2 we obtained the identities of following type:

$$
\operatorname{ch}_{\bigwedge\left(W_{N}^{\mathbf{Z}+h,-}\right)}\left[-\tau D_{0}+\mathrm{L}(\mathbf{u})+\mathrm{R}(\mathbf{v})\right]=\sum_{Y} \chi_{\hat{\lambda}_{\mathrm{L}}(Y), R}^{\mathrm{L}}(\tau, \mathbb{u}) \chi_{\hat{\lambda}_{\mathbf{R}}(Y), L}^{\mathrm{R}}(\tau, \mathbf{v})
$$

(Remark 4.2(ii)). Here we use the notation in Table 4.2 and for simplicity write $\chi_{*}^{\mathrm{L}}$,

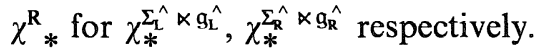

It is well known that the vector space spanned by normalized characters for integrable highest weight modules is stable under the modular transformation (see e.g. $\left[\mathrm{KW}\right.$, Lemma 2.1]). As is easily seen from the fact $\#\left(\Sigma_{\mathrm{L}}^{\wedge}\right)<\infty$ (that is, we can consider the mean with respect to the Haar measure of $\Sigma_{\mathbf{L}}^{\wedge}$ ) this is also true for our characters $\chi^{\mathrm{L}}{ }_{*}$ for the system $\Sigma_{\hat{L}}^{\wedge} \ltimes \mathrm{g}_{\hat{\mathrm{L}}}$ of Case $\hat{\mathrm{C}} \hat{\mathrm{C}}, \hat{\mathrm{B}} \hat{\mathrm{B}}, \hat{\mathrm{D}} \hat{\mathrm{B}}$ and $\hat{\mathrm{D}} \hat{\mathrm{D}}$ in Table 4.2. Let $m^{\mathrm{L}}=\left(m^{\mathrm{L}}{ }_{Y, y}\right)_{Y, y \in \mathscr{Y}_{l, r}}$ and $m^{\mathrm{R}}=\left(m^{\mathrm{R}}{ }_{Y, y}\right)_{Y, y \in \mathscr{Y}_{l, r}}$ respectively be the scalar matrices of the

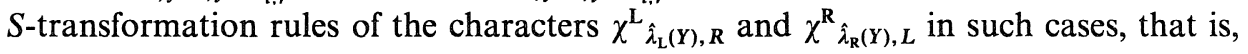

$$
\begin{aligned}
& \chi^{\mathrm{L}}{\hat{\lambda_{L}(Y), R}} \circ S=\sum_{y \in \mathscr{Y}_{l, r}} m_{Y, y}^{\mathrm{L}} \chi_{\hat{\lambda}_{L}(y), R}^{\mathrm{L}}, \\
& \chi^{\mathrm{R}}{\hat{\hat{\lambda}_{\mathrm{R}}(Y), L}} \circ S=\sum_{y \in \mathscr{Y}_{l, r}} m^{\mathrm{R}}{ }_{Y, y} \chi_{\hat{\lambda}_{\mathrm{R}}(y), L}^{\mathrm{R}} .
\end{aligned}
$$

Note that these definitions of $m^{\mathrm{L}}$ and $m^{\mathrm{R}}$ depend on the choice of $h(=0,1 / 2)$, since $\hat{\lambda}_{\mathrm{L}}$ and $\hat{\lambda}_{\mathbf{R}}$ depend.

In view of Proposition 6.1, the identity (1) gives the following information on the transformation rules of characters.

Corollary 6.2a. For the pair $\left(\mathfrak{g}_{\hat{L}}^{\wedge}, \mathfrak{g}_{\hat{R}}\right)$ in Table $4.2(h=1 / 2) \hat{\mathrm{C}} \hat{C}, \hat{\mathrm{B}} \hat{\mathrm{B}}, \hat{\mathrm{B}} \hat{\mathrm{D}}$ and $\hat{\mathrm{D}} \hat{\mathrm{D}}$, there is a "duality"

$$
\sum_{\mathbf{Y} \in \mathscr{Y}_{l, r}} m^{\mathrm{L}}{ }_{\mathbf{Y}, y^{\prime}} m^{\mathrm{R}}{ }_{\mathbf{Y}, y^{\prime}}=\delta_{y, y^{\prime}} \quad \text { for } \quad y, y^{\prime} \in \mathscr{Y}_{l, r} .
$$

Proof. Remark that $\operatorname{ch}_{N}^{+, Z+1 / 2}$ is invariant under $S$ (Table 6.1(a), (e)). Hence together with (1) we have the following.

$$
\begin{aligned}
& \operatorname{ch}_{N}^{+, Z+1 / 2}=\sum_{Y} \chi_{\hat{\lambda}_{L}(Y), R}^{\mathrm{L}} \chi_{\hat{\lambda}_{\mathrm{R}}(Y), L}^{\mathrm{R}}=\operatorname{ch}_{N}^{+, Z+1 / 2} \circ S=\sum_{Y}\left(\chi_{\hat{\lambda}_{\mathrm{L}}(Y), R}^{\mathrm{L}} \circ S\right)\left(\chi_{\hat{\lambda}_{\mathrm{R}}(Y), L}^{\mathrm{R}} \circ S\right) \\
& =\sum_{Y}\left(\sum_{y} m_{Y, y}^{\mathrm{L}} \chi_{\hat{\lambda}_{L}(y), R}^{\mathrm{L}}\right)\left(\sum_{y^{\prime}} m_{Y, y^{\prime}}^{\mathrm{R}} \chi_{\hat{\lambda}_{\mathrm{R}}\left(y^{\prime}\right), L}^{\mathrm{R}}\right)
\end{aligned}
$$


as a function on $\left(\mathfrak{h}^{\mathfrak{g}_{\mathrm{L}}} \oplus \mathfrak{h}^{\mathfrak{g}_{\mathrm{R}}} \oplus \mathbb{C} \boldsymbol{c} \oplus \mathbb{C} d\right) \subset \tilde{\mathfrak{h}}^{\mathfrak{o}(N)^{\wedge}}$. The claim now follows from the linear independence of characters.

The group of auxiliary automorphisms $\Sigma_{L} \times \Sigma_{R}$ in (1) is trivial for the Case $\hat{C} \hat{C}$. Therefore the well known unitarity of the matrices $m^{\mathrm{L}}$ and $m^{\mathrm{R}}([\mathrm{KW}$, Lemma 2.1]) allows us to write (3) as $m_{Y, y}^{\mathrm{L}}=\overline{m_{Y, y}^{\mathrm{R}}}$ in this case, where the bar denotes the complex conjugation.

On the other hand, in the Case $\hat{A} \hat{A}$, unfortunately the space spanned by $\chi_{(Y,[Y]), r}^{\mathbb{Z}^{l} \times g(l) \wedge}$ (1.3.4(14)) is not stable under the modular transformation. However a similar result associated to the triple $\mathbb{C l}^{\wedge} \oplus \mathfrak{s l}(l)^{\wedge} \oplus \mathfrak{s l}(r)^{\wedge}$ is of course available as follows. Note that the functions $\theta_{j, n}^{\mathrm{Z}}(\tau, u) / \eta(\tau)(0 \leq j \leq n)$ again spans a invariant subspace of the $S$-transformation (1.5.1(8)).

Corollary 6.2b. Define matrices $m_{j, k}^{\mathrm{Cl}}, m_{\lambda, \lambda^{\prime}}^{\mathrm{L}}$ and $m_{\mu, \mu^{\prime}}^{\mathrm{R}}$ by

$$
\begin{aligned}
& \left(\theta^{\mathbb{Z}}{ }_{j, l} / \eta\right) \circ S=\sum_{k=1}^{l r} m_{j, k}^{\mathbb{C l}}\left(\theta_{k, l r}^{\mathbb{Z}} / \eta\right), \\
& \chi_{\lambda, r}^{\mathfrak{s l}(l)^{\wedge}} \circ S=\Sigma_{\lambda^{\prime} \in \mathscr{Y}_{l-1, r}} m_{\lambda, \lambda^{\prime}}^{\mathrm{L}} \chi_{\lambda^{\prime}, r}^{\mathfrak{s l}(l)^{\wedge}}, \\
& \chi_{\mu, l}^{\mathfrak{S I}(r)^{\wedge}} \circ S=\Sigma_{\mu^{\prime} \in \mathscr{Y}_{r-1, l}} m^{\mathrm{R}}{ }_{\mu, \mu^{\prime}} \chi_{\mu^{\prime}, l}^{\mathfrak{I I}(r)^{\wedge}},
\end{aligned}
$$

then it holds that

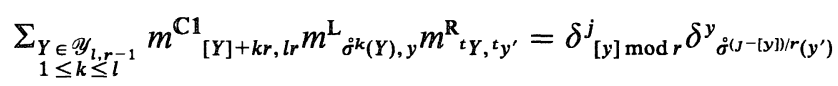

for $y \in \mathscr{Y}_{l-1, r}, y^{\prime} \in \mathscr{Y}_{l, r-1}$ and $1 \leq j \leq r$, where for $Y \in \mathscr{Y}_{l, r-1} \subset \mathfrak{h}^{* \mathrm{sI}(l)}$ we define $\stackrel{\circ}{ }(Y)$ by

$$
\sigma(Y)+r \hat{\Lambda}_{0} \equiv \sigma_{c y c}\left(Y+r \hat{\Lambda}_{0}\right) \bmod \mathbf{C} \delta .
$$

Proof. Note that the character identity of Theorem 4.2 for Case $\hat{A} \hat{A}$ can be rewritten as the followings (4.4(3)):

$$
\operatorname{ch}_{2 l r}^{\mathrm{Z}+1 / 2}=\sum_{\substack{Y \in \mathscr{Y}_{l, r}-1 \\ 1 \leq k \leq r}}\left(\frac{1}{\eta} \theta_{[Y]+l k, l r}^{\mathbb{Z}}\right) \cdot \chi_{\sigma_{c y c}^{k}}^{\mathrm{II}(r)} \hat{(Y, l)} \cdot \chi_{t Y, r}^{\mathrm{I}(l) \wedge},
$$

and then apply the argument in the proof of Cor. 6.2a.

Remark 6.2. Besides the above results Cor. $6.2 \mathrm{a}, 2 \mathrm{~b}$ which are based on $\mathrm{ch}_{N}^{+}, \mathrm{Z}+1 / 2$, we can also obtain a similar results from $\mathrm{ch}_{2 n}^{-,}$: Table $6.1(\mathrm{~h})$ says

$$
\operatorname{ch}_{2 n}^{-, Z} \circ S=(-i)^{n} \operatorname{ch}_{2 n}^{-,}, \mathbf{Z}
$$

Here we apply this to the character identity of Case $\hat{C} \hat{C}(h=0)$ of Theorem 4.2 , for example. For that purpose we read from List 4.2 the information that wheather each component $\mathbb{L}^{\mathfrak{s p}(2 l)^{\wedge}}(Y, r) \otimes \mathbb{L}^{\mathfrak{s p}(2 r)^{\wedge}}\left(Y^{\dagger}, l\right)$ belongs to $\bigwedge^{\text {even }}\left(W_{4 l r}^{\mathbb{Z},-}\right)$ or $\bigwedge^{\text {odd }}\left(W_{4 l r}^{\mathbb{Z},-}\right)$, and obtain

$$
\operatorname{ch}_{4 l r}^{-, Z}=\Sigma_{Y \in \mathscr{Y}_{l, r}}(-1)^{\left[Y^{\dagger}\right]} \chi_{Y, r}^{\mathfrak{s p}(2 l)^{\wedge}} \chi_{Y^{\dagger}, l}^{\mathfrak{s p}(2 r)^{\wedge}} .
$$

Now we apply the $S$-transformation to both hand sides and use (10), to deduce the following: for $y, y^{\prime} \in \mathscr{Y}_{l, r}$, 


$$
\Sigma_{Y \in \mathscr{Y}_{l, r}}(-1)^{\left[Y^{\dagger}\right]} M_{Y, y}^{(l, r)} M_{Y^{\dagger}, y^{\prime+}}^{\left(r_{+} l\right)}=(-i)^{2 l r}(-1)^{\left[y^{\dagger}\right]} \delta^{y}{ }_{y^{\prime}}=(-1)^{[y]} \delta^{y} y^{\prime},
$$

where $M_{Y, y}^{(l, r)}:=m_{Y, y}^{\mathrm{L}}$, that is,

$$
\chi_{Y, r}^{\mathfrak{s p}(2 l)^{\wedge}} \circ S=\Sigma_{y \in \mathscr{Y}_{l, r}} M_{Y, y}^{(l, r)} \chi_{y, r}^{\mathfrak{s p}(2 l)^{\wedge}} .
$$

\section{§7. Are There Affine Segal-Shale-Weil Versions?}

7.0. Here we seek the symmetric algebra analogues of our dual pairs and obtain a negative answer.

7.1. We have shown the spin module versions of Weyl's reciprocity theoremdual pairs on spinors-for classical, finite or non-twisted affine type Kac-Moody Lie algebras (Theorems 3.2 and 4.2). As an application, the duality of branching rules, or coset Virasoro modules, are explained from the viewpoint of seesaw dual pairs (Section 5).

Now we consider the problem, whether there is a Segal-Shale-Weil analogue of our results. Namely, if whether there are dual pairs of affine Lie algebras on affine SegalShale-Weil module $\mathscr{S}=\mathscr{S}_{n}$ of $\mathfrak{s p}(2 n)^{\wedge}$, which is constructed by Feingold-Frenkel [FF] on the symmetric algebra $\mathscr{S}$ of a certain infinite dimensional vector space. Since our picture is quite similar to that of [Ho] and the literature cited there, which concern with dual pairs on the Segal-Shale-Weil modules over $\mathfrak{s p}(2 l)$, it is natural to seek such possibility. As in the finite dimensional case, we will consider the module $\mathscr{S}=\mathscr{S}_{l r}$ as the module of the following subalgebras of $\mathfrak{s p}(2 l r)^{\wedge}$ :

$$
\begin{aligned}
& \mathfrak{g l}(l)^{\wedge} \oplus \mathfrak{s l}(r)^{\wedge} \subset \mathfrak{s p}(2 l r)^{\wedge}, \\
& \mathfrak{s p}(2 l)^{\wedge} \oplus \mathfrak{v}(r)^{\wedge} \subset \mathfrak{s p}(2 l r)^{\wedge} .
\end{aligned}
$$

What we have to check first is whether the central charge identities, which play an essential role in our previous consideration on the spinor cases $(\S 0.3)$, are satisfied for the above pairs $(1,2)$. Let us denote by $z^{\mathfrak{g}^{\wedge}}$ the central charge of the Virasoro operators associated to the representation of $\mathrm{g}^{\wedge}$ on $\mathscr{S}_{l r}(\S 1.2 .2)$. The answer is then the followings:

Lemma 7.1. There are no positive integer solutions $(l, r) \in \mathbb{Z}_{>0} \times \mathbb{Z}_{>0}$ (resp. $\mathbb{Z}_{>0} \times$ $\mathbf{Z}_{>1}$ ) for the following equations (3) (resp. (4)).

$$
\begin{aligned}
& z^{\mathfrak{s p}(2 l r)^{\wedge}}=z^{\mathfrak{g l}(l) \wedge}+z^{\mathfrak{s l}(r)^{\wedge}} \\
& z^{\mathfrak{s p}(2 l r)^{\wedge}}=z^{\mathfrak{s p}(2 l)^{\wedge}}+z^{\mathfrak{O}(r)^{\wedge}}
\end{aligned}
$$

Proof. Recall that the module $\mathscr{S}$ is of level $(-1)$ as the $\mathfrak{s p}(2 n)^{\wedge}$-module (therefore it is not integrable $[\mathrm{FF},(6.1)]$ ). Taking the ratios of the normalized bilinear forms of $\mathfrak{s p}(2 l r)$ and that of $\mathfrak{s l}(l)$ and $\mathfrak{s l}(r)$ into consideration, (3) reads as

$$
\frac{-1 \cdot \operatorname{lr}(2 l r+1)}{-1+(l r+1)}=\left(1+\frac{-2 r\left(l^{2}-1\right)}{-2 r+l}\right)+\frac{-2 l\left(r^{2}-1\right)}{-2 l+r},
$$


which reduces to

$$
(1 / r-1 / l)^{2}=3-1 / l r .
$$

Then noting $(1 / r-1 / l)^{2}<1$ for $(l, r) \in \mathbb{Z}_{>0}{ }^{2}$ we assert the statement for (3).

As for (4), the equation is equivalent to

$$
\frac{-1 \cdot \operatorname{lr}(2 l r+1)}{-1+(l r+1)}=\frac{-r\left(2 l^{2}+l\right)}{-r+(l+1)}+\frac{-4 l \cdot\left(r^{2}-r\right) / 2}{-4 l+(r-2)},
$$

which reduces to

$$
(3 l r+2 l-r+2)(2 l+1)(r-1)=0 .
$$

Then we solve $3 l r+2 l-r+2=0$ and get $r=-2(l+1) /(3 l-1)$, which is impossible for $(l, r) \in \mathbb{Z}_{>0}{ }^{2}$.

Therefore we consider the coset Virasoro operators and conclude (unfortunately)

Proposition 7.1. For the pair

$\left(1^{\prime}\right)\left(\mathfrak{g l}(l)^{\wedge}, \mathfrak{s l}(r)^{\wedge}\right) \quad\left(\operatorname{resp} \cdot\left(2^{\prime}\right):\left(\mathfrak{s p}(2 l)^{\wedge}, \mathfrak{o}(r)^{\wedge}\right)\right)$

of Lie algebras, assume the following condition (5) (resp. (6)). Then there are non-zero Virasoro operators (i.e. a non-trivial action of the Virasoro algebra) which commute with the algebra pair $\left(1^{\prime}\right)$ (resp. $\left.\left(2^{\prime}\right)\right)$ acting on the affine Segal-Shale-Weil module $\mathscr{S}_{l r}((1),(2))$. In particular, $\left(1^{\prime}\right)\left(\right.$ resp. $\left.\left(2^{\prime}\right)\right)$ does not form a dual pair on $\mathscr{S}_{l r}$.

$$
\begin{aligned}
2 r \neq l & \text { and } & 2 l \neq r . \\
r \neq l+1 & \text { and } & 4 l \neq r-2 .
\end{aligned}
$$

Proof. Under the above assumption (5), the coset Virasoro operators $D^{\mathfrak{s p}(2 l r)^{\wedge}}(n)-$ $\left(D^{\mathrm{gl}(2 l)^{\wedge}}(n)+D^{\mathrm{sl}(r)^{\wedge}}(n)\right)(n \in \mathbb{Z})$ are well-defined and cannot be zero because its central charge does not vanish. This proves the proposition for the pair $\left(\operatorname{gl}(l)^{\wedge}, \mathfrak{s l}(r)^{\wedge}\right)$. The statement for $\left(\mathfrak{s p}(2 l)^{\wedge}, \mathfrak{o}(r)^{\wedge}\right)$ can be obtained similarly.

If (5) or (6) is not satisfied in each case, the Sugawara form does not define a representation of the Virasoro algebra. See also [KK] or [Hay] for such a case.

\section{\$ \&. Appendix: The Fermion-Boson Correspondence}

8.0. In this appendix we review the Fermion-Boson correspondence ([DJKM], [F2]) and give an explicit description of the action of the outer automorphism $\sigma_{c y c}^{\#}$ in the Case $\hat{A} \hat{A}$ of Theorem 4.2 .

\subsection{The Fermion-Boson correspondence}

We follow the notations in $1.3 .3,1.3 .4,1.4 .5$, and $2.3 \sim 4$. Consider the homogeneous Heisenberg subalgebra

$$
\mathfrak{h}^{\wedge}=\left(\bigoplus_{k=1}^{n} \bigoplus_{m \in \mathbb{Z}} \mathbb{C D}^{k}(m)\right) \oplus \mathbb{C} c
$$

of $\mathfrak{o}(2 n)^{\wedge}$ and the lattice $\mathbb{Z}^{n}=\bigoplus_{j=1}^{n} \mathbb{Z} \varepsilon_{j} \subset \mathfrak{h}^{*}$. Each $\gamma \in \mathbb{Z}^{n}$ acts on $\hat{\mathfrak{h}}$ by 


$$
t_{\gamma}(x(m))=x(m)-\delta_{m, 0} \gamma(x) c \quad(x(m) \in \hat{\mathfrak{h}}) \quad \text { and } \quad t_{\gamma}(c)=c,
$$

and the pair $\mathbb{Z}^{n} \ltimes \hat{\mathfrak{h}}$ forms a Heisenberg system (1.3.3(1)). Let $\varepsilon: \mathbb{Z}^{n} \times \mathbb{Z}^{n} \rightarrow\{ \pm 1\}$ be the 2-cocycle defined by

(3) $\varepsilon\left(\varepsilon_{j}, \varepsilon_{k}\right):=\left\{\begin{aligned} 1 & \text { if } j \leq k \\ -1 & \text { if } j>k\end{aligned}\right.$ and $\varepsilon(\alpha+\beta, \lambda+\mu)=\varepsilon(\alpha, \lambda) \varepsilon(\alpha, \mu) \varepsilon(\beta, \lambda) \varepsilon(\beta, \mu)$.

Then for each $\lambda \in \mathfrak{h}^{*}$ an irreducible representation of $\mathbb{Z}^{n} \ltimes \hat{\mathfrak{h}}$ associated to $\varepsilon$ is defined on the Fock space $[\mathrm{FK}]$

$$
\mathbb{L}^{\mathbf{Z}^{n} \ltimes \hat{h}}(\lambda ; l) \simeq e^{\lambda} \cdot \bigotimes_{k=1}^{n} \mathbb{C}\left[e^{l \varepsilon_{k}}, e^{-l \varepsilon_{k}}, x_{k}(1), x_{k}(2), \ldots\right]
$$

by the formulae $1.3 .3(4)$.

On the other hand, putting $\check{h}:=1 / 2-h(h=0,1 / 2)$, for each $\gamma \in \mathbb{Z}^{n}$ we can define a vector $\left|\gamma+\check{h} \mathbb{H}^{*}\right\rangle \in \bigwedge\left(W_{2 n}^{\mathbf{Z}+h,-}\right)$ inductively by the rules

$$
\begin{gathered}
\left|\check{h} \mathbb{1}^{*}\right\rangle:=|v a c\rangle:=1 \in \bigwedge\left(W_{2 n}^{\mathbb{Z}+h,-}\right) \\
\left|\gamma \pm \varepsilon_{k}+\check{h} \mathbb{1}^{*}\right\rangle:=\varepsilon\left(\varepsilon_{k}, \gamma\right) \psi^{ \pm k}\left(\mp\left(\gamma \mid \varepsilon_{k}\right)-h \mp \check{h}\right)\left|\gamma+\check{h} \mathbb{1}^{*}\right\rangle,
\end{gathered}
$$

where $\left(\varepsilon_{j} \mid \varepsilon_{k}\right):=\delta_{j k}, \mathbb{1}^{*}:=\sum_{j=1}^{n} \varepsilon_{j}=2 \Lambda_{n}$ and $\psi^{ \pm k}(\mu)=\psi_{\mp k}(\mu)$ are the basis of $W_{2 n}^{\mathbf{Z}+h}$ given in $\S \S 2.3 \sim 2.4$ that satisfy the canonical anticommutation relation

$$
\begin{aligned}
& \left\{\psi^{j}(\mu), \psi_{k}(v)\right\}=\delta^{j}{ }_{k} \delta_{\mu+v, 0} \\
& \left\{\psi^{j}(\mu), \psi^{k}(v)\right\}=0=\left\{\psi_{j}(\mu), \psi_{k}(v)\right\}
\end{aligned}
$$

for $1 \leq j, k \leq n$ and $\mu, v \in \mathbb{Z}+h$. Similarly to (5) we define a vector $\left\langle\gamma+\check{h} \mathbb{1}^{*}\right|$ of $\bigwedge\left(W_{2 n}^{\mathrm{Z}+h,+}\right)$ by

$$
\begin{aligned}
\left\langle\check{h} \mathbb{1}^{*}\right| & :=\langle v a c|:=1 \in \bigwedge\left(W_{2 n}^{\mathrm{Z}+h,+}\right), \\
\left\langle\gamma \pm \varepsilon_{k}+\check{h} \mathbb{1}^{*}\right| & :=\left\langle\gamma+\check{h} \mathbb{1}^{*}\right| \varepsilon\left(\varepsilon_{k}, \gamma\right) \psi_{ \pm k}\left( \pm\left(\gamma \mid \varepsilon_{k}\right)+h \pm \check{h}\right) .
\end{aligned}
$$

From now on we identify $\bigwedge\left(W_{2 n}^{\mathrm{Z}+h,+}\right)$ with the right $C\left(W_{2 n}^{\mathrm{Z}+h}\right)$-module $W_{2 n}^{\mathbb{Z}+h,-} C\left(W_{2 n}^{\mathbf{Z}+h}\right) \backslash C\left(W_{2 n}^{\mathbf{Z}+h}\right)$ as in $\S 2.2$. Then there is a non-degenerate pairing

$$
\langle\mid\rangle: \bigwedge\left(W_{2 n}^{\mathbf{Z}+h,+}\right) \times \bigwedge\left(W_{2 n}^{\mathbf{Z}+h,-}\right) \rightarrow \mathbf{C}
$$

which is uniquely determined by $\langle 1 \mid 1\rangle:=1$ and

$$
\left\langle u_{+} a \mid u_{-}\right\rangle=\left\langle u_{+} \mid a u_{-}\right\rangle\left(=:\left\langle u_{+}|a| u_{-}\right\rangle\right)
$$

for any $u_{ \pm} \in \bigwedge\left(W_{2 n}^{\mathbf{Z}+h, \mp}\right)$ and $a \in C\left(W_{2 n}^{\mathbf{Z}+h}\right)$.

Now we have the following $\mathbb{Z}^{n} \propto \hat{\mathfrak{b}}$-isomorphism, which is equivalent to the Jacobi triple product formula.

Theorem 8.1. (The Fermion-Boson correspondence [DJKM]) Put

$$
H(x):=\sum_{m \in \mathbb{Z}_{>0}} \sum_{1 \leq k \leq n} m^{-1} x_{k}(m) D_{k}^{k}(m) .
$$

Then we have the following $\hat{\mathfrak{h}}$-isomorphism. 


$$
\begin{aligned}
& \mathscr{B}: \bigwedge\left(W_{2 n}^{\mathbf{Z}+h,-}\right) \stackrel{\sim}{\rightarrow} \mathbb{L}^{\mathbf{Z}^{n} \times \hat{h}}\left(\check{h} \mathbb{1}^{*}, 1\right) \\
& \Psi \quad \omega \\
& |v\rangle \quad \mapsto \sum_{\gamma \in \mathbb{Z}^{n+\zeta} 1^{*}} e^{\gamma} \otimes\left\langle\gamma\left|e^{H(x)}\right| v\right\rangle .
\end{aligned}
$$

Moreover $\mathscr{B}$ gives rise to a $\mathbb{Z}^{n} \ltimes \hat{\mathfrak{h}}$-isomorphism if we define the projective action $T_{\alpha}^{\sim}$ of $\alpha \in \mathbb{Z}^{n}$ on $\bigwedge\left(W_{2 n}^{\mathbf{Z}+h,-}\right)$ by

$$
T_{\alpha}^{\sim}\left(\Sigma_{\gamma} P_{\gamma}|\gamma\rangle\right):=\Sigma_{\gamma} P_{\gamma} \cdot \varepsilon(\alpha, \gamma)|\alpha+\gamma\rangle \quad\left(P_{\gamma} \in U\left(\mathfrak{h} \otimes \mathbb{C}\left[t^{-1}\right]\right)\right) .
$$

This isomorphism leads to the "vertex operator representation" of $\psi_{ \pm k}(v)$. Put $\hat{a}(z):=\sum_{v \in \mathbf{Z}+h} a(v) z^{-v-1 / 2}$ for $a \in W_{2 n}$

Proposition 8.1 ([F2] p. 77).

$$
\hat{\psi}_{ \pm k}(z)=e^{ \pm Q_{\bar{k}}(z)} e^{ \pm Q_{k}^{+}(z)} T_{ \pm \varepsilon_{k}}^{\sim} z^{ \pm D^{k_{k}}},
$$

where $Q_{k}^{ \pm}(z):=\sum_{n=1}^{\infty} \frac{z^{\mp n}}{\overline{\mp n}} D_{k}^{k}( \pm n)$.

\subsection{An explicit description of the automorphism $\sigma_{c y c}^{\#}$}

An index changing induces the action of $w \in \mathcal{S}_{n}$ on $\mathbb{Z}^{n}$ and $\bigotimes_{k=1}^{n} \mathbb{C}\left[x_{k}(1), \ldots\right]$ by

$$
w\left(\varepsilon_{k}\right):=\varepsilon_{w(k)} \quad \text { and } \quad w\left(P\left(\ldots, x_{k}(m), \ldots\right)\right):=P\left(\ldots, x_{w(k)}(m), \ldots\right),
$$

where $P \in \bigotimes_{k} \mathbf{C}\left[x_{k}(1), \ldots\right]$ and $1 \leq k \leq n$. We will twist this action to have desirable commutation relations with $T_{\gamma}$ 's. Fix $w \in \mathfrak{S}_{n}$, and give $\varepsilon_{w}\left(\varepsilon_{j}\right) \in\{ \pm 1\}$ for $1 \leq j \leq n$. We can extend $\varepsilon_{w}$ inductively and uniquely to a map $\varepsilon_{w}: \mathbb{Z}^{n} \rightarrow\{ \pm 1\}$ by the following:

$$
\varepsilon_{w}(0):=1 \quad \text { and } \quad \varepsilon_{w}\left(\gamma \pm \varepsilon_{k}\right):=\varepsilon\left(w\left(\varepsilon_{k}\right), w(\gamma)\right) \varepsilon\left(\varepsilon_{k}, \gamma\right) \varepsilon_{w}\left(\varepsilon_{k}\right) \varepsilon_{w}(\gamma)
$$

for any $\gamma \in \mathbb{Z}^{n}$ and $k=1, \ldots, n$. Using this $\varepsilon_{w}$ we can define $\tilde{w} \in \operatorname{Aut}\left(\mathbb{L}^{\mathbf{Z}^{n} \ltimes \hat{h}}(\lambda, 1)\right)$ by

$$
\tilde{w}\left(e^{\lambda+\gamma} P(x)\right):=\varepsilon_{w}(\gamma) e^{w(\lambda+\gamma)} \cdot w(P(x))
$$

for $e^{\lambda+\gamma} P(x) \in \mathbb{L}^{\mathbf{Z}^{n} \ltimes \hat{h}}(\lambda, 1)$. Then the following formulae follows by the definition.

Lemma 8.2. For any $\gamma \in \mathbb{Z}^{n}, n \in \mathbb{Z}_{>0}$ and $k=1, \ldots, n, \tilde{w} T_{\gamma}^{\sim} \tilde{w}^{-1}=\varepsilon_{w}(\gamma) T_{w(\gamma)}^{\sim}$, $\tilde{w}\left(\partial / \partial \varepsilon_{k}\right) \tilde{w}^{-1}=\partial / \partial \varepsilon_{w(k)}, \tilde{w}\left(x_{k}(m)\right) \tilde{w}^{-1}=x_{w(k)}(m)$ and $\tilde{w}\left(\partial / \partial x_{k}(m)\right) \tilde{w}^{-1}=\partial / \partial x_{w(k)}(m)$.

These projective actions for $M \subset \mathbb{Z}^{n}(M$ denotes the root lattice of $\mathfrak{o}(2 n), 1.5 .5(2))$ and $\mathfrak{S}_{n}$ can be identified with that of the Weyl group of $\mathfrak{o}(2 n)^{\wedge}$, which is defined through the integrable representation [FK].

Now we will give the projective action $\tilde{\sigma}_{c y c}^{\#}$ of the outer automorphism $\sigma_{c y c}^{\#}(1.3 .4(1))$ of $\operatorname{gl}(l)^{\wedge}$ on $\bigwedge\left(W_{2 n}^{\mathbf{Z}+h,-}\right)$ that appears in Case $\hat{\mathrm{A}} \hat{\mathrm{A}}$ of Theorem 4.2. We put $n=l r$ and use the index $(j, p)(1 \leq j \leq l, 1 \leq p \leq r)$ instead of $1 \leq k \leq n$. Put $\gamma_{1}:=\Sigma_{p=1}^{r} \varepsilon_{1, p}$ and let $T_{\gamma_{1}}^{\sim}$ be the corresponding twisted translation operator associated to the cocycle $\varepsilon$ (8.1.(3)) and $(1, \ldots, l)^{\sim}$ the twisted action (13) of the cycle

$$
\mathfrak{S}_{l x} \ni(1, \ldots, l):(j, p) \mapsto(j+1 \bmod l, p)
$$


(any $\varepsilon_{w}$ will do). Then identifying the Fermion Fock space $\bigwedge\left(W_{2 l r}^{\mathbf{Z}+h,-}\right)$ and the Boson Fock space $\mathbb{L}^{\mathbb{Z}^{n} \times \hat{h}}\left(\breve{h}^{*}, 1\right)$ by $\mathscr{B}$ in Theorem 8.1 , we define

$$
\tilde{\sigma}_{c y c}^{\#}:=T_{\gamma_{1}}^{\sim} \cdot(1, \ldots, l)^{\sim} \in \text { Aut } \bigwedge\left(W_{2 l r}^{\mathbf{Z}+h,-}\right) .
$$

Proposition 8.2. For any $g \in \mathfrak{g l}(l)^{\wedge}, \tilde{\sigma}_{c y c}^{\#}$ satisfies

$$
\tilde{\sigma}_{c y c}^{\#} \cdot \hat{S} \circ \hat{L}(g) \cdot \tilde{\sigma}_{c y c}^{\#-1}=\hat{S} \circ \hat{L}\left(\sigma_{c y c}^{\#}(g)\right)
$$

on $\bigwedge\left(W_{2 l r}^{\mathbf{Z}+h,-}\right)$. It also satisfies

$$
\left(\tilde{\sigma}_{c y c}^{\#}\right)^{l}= \pm T^{\sim}\left(\sum_{\jmath, p}^{\varepsilon_{j, p}}\right) .
$$

Proof. Apply Lemma 8.2 to the vertex operator representation given by Proposition 8.1.

\section{References}

[B] N. Bourbaki, Group et algèbre de Lie (Chap. 7 \& 8), Hermann, Paris, 1975.

[DJKM] E. Date, M. Jimbo, M. Kashiwara and T. Miwa, Transformation groups for soliton equations, In Proceedings of 1981 RIMS Symposium, M. Jimbo and T. Miwa eds, World Scientific (1983).

[DJKMO] E. Date, M. Jimbo, A. Kuniba, T. Miwa and M. Okado, Exactly solvable SOS Models II: Proof of the star-triangle relation and combinatrial identities, Advanced studies in pure math., 16 (1988), 17-122.

$[\mathrm{FFu}]$ B. L. Feigin and D. B. Fuchs, Verma modules over the Virasoro algebra, Springer LNM., 1060 (1984), 230-245.

[FF] A. Feingold and I. Frenkel, Classical affine algebras, Adv. Math., 56 (1985), 117-172.

[F1] I. B. Frenkel, Spinor representation of affine Lie algebra, Proc. Nat'l. Acad. Sci. USA, 77 (1980), 6303-6306.

[F2] — Representations of affine Lie algebras, Hecke modular forms, and KdV type equations, Springer LNM., 933 (1982), 71-110.

[FK] I. B. Frenkel and V. G. Kac, Basic representations of affine Lie algebras and dual resonance models, Inv. Math., 62 (1980), 23-66.

[GKO] P. Goddard, A. Kent and D. Olive, Unitary representations of the Virasoro and super Virasoro algebras, Commun. Math. Phys., 103 (1986), 105-119.

[Has] K. Hasegawa, Dual pairs on spinors-cases of $\left(C_{m}, C_{n}\right)$ and $\left(C_{m}^{(1)}, C_{n}^{(1)}\right)$, Proc. Japan Acad., 63, Ser. A (1987), 400-403.

[Hay] T. Hayashi, Sugawara operators and Kac-Kazhdan conjecture, Inv. Math., 94 (1988), 13-52.

[Ho] R. Howe, Dual pairs in Physics, Lectures in Applied Math., 21, AMS (1985), 179-207.

[Ho1] - Transcending classical invariant theory, J. Amer. Math. Soc., 2 (1989), 535-552.

[JM] M. Jimbo and T. Miwa, On a duality of branching rules for affine Lie algebras, Advanced studies in Pure Math., 6 (1985), 17-65.

[JM1] — On a duality of branching coefficients, in "Vertex operators in Mathematics and Physics", Proceeding of a conference Nov. 10-17, 1983, Publ. MSRI 3, Springer (1984).

[Kac] V. G. Kac, Infinite dimensional Lie algebras, 2nd ed., Cambridge Univ. Press, 1985.

[KK] V. G. Kac and D. A. Kazhdan, Structure of representations with highest weight of infinite dimensional Lie algebras, Adv. Math., 34 (1979), 97-108.

[KP1] V. G. Kac and D. Peterson, Spin and wedge representations of infinite-dimensional Lie algebras and groups, Proc. Nat'l. Acad. Sci., USA. 78 (1981), 3308-3312.

[KP2] — Infinite dimensional Lie algebras, theta functions and the modular forms, Adv. Math. 53 (1984), 125-264. 
[KW] V. G. Kac and M. Wakimoto, Modular and conformal invariance constraints in representation theory of affine Lie algebras, Adv. Math., 70 (1988), 156-236.

[KNTY] N. Kawamoto, Y. Namikawa, A. Tsuchiya and Y. Yamada, Geometric realization of conformal field theory on Riemann surfaces, Comm. Math. Phys., $\mathbb{1 1 6}$ (1988), 247-308.

[Ko] K. Koike, On the decomposition of tensor products of the representations of the classical groups, to appear in Adv. Math.

[KoT1] K. Koike and I. Terada, Young diagammatic methods for representation theory of the classical groups of type $B_{n}, C_{n}, D_{n}$. J. Alg., 107 (1987), 466-511.

[KoT2] — Y Young diagammatic methods for the restriction of complex classical Lie groups to reductive subgroups of maximal rank, to appear in Adv. Math.

[Ku] S. Kudla, Seesaw dual reductive pairs. in Proceedings of 1983 Taniguchi Symposium "Automorphic Forms of Several Variables", I. Satake and Y. Morita eds., pp 244-268, Birkhauser (1984).

[Mac] I. G. Macdonald, Symmetric functions and Hall polynomials, Oxford Univ. Press, 1979.

[P] R. Parthasarathy, Dirac operators and the discrete series, Ann. Math., 96 (1972), 1-30.

[R] G. de B. Robinson, Representation theory of the symmetric group, University of Toronto Press, 1961.

[Se] J.-P. Serre, Cours d'arithmetique, Presses Univ., France, Paris, 1970.

[SN] M. Sato and M. Noumi, Soliton equation and universal Grassmann manifold, Sophia Univ. Kôkyuroku, 18 (in Japanese), 1984.

[TK1] A. Tsuchiya and Y. Kanie, Unitary representations of the Virasoro algebra, Duke Math. J., 53, (1986), 1013-1046.

[TK2] - Unitary representations of the Virasoro algebra and branching law of representations of affine Lie algebras, in Proceedings of Taniguchi symposium 1985 at Katata, K. Ito and N. Ikeda eds., Kinokuniya (1987), 385-419.

[Ya] I. Yamanaka, Equivalence of degenerate (super) conformal models, Prog. Theor. Phys., 76 (1986), 1154-1165.

[W] H. Weyl, The classical groups, Princeton Univ. Press, 1939.

Note added

After the submission of this paper, the author come to know the following papers which are closely related to the present paper:

P. Goddard, W. Nahm and D. Olive, Symmetric spaces, Sugawara's energy momentum tensor in two dimensions and free fermions, Phys. Lett, 160B, (1985), 111-116.

W. Nahm, Lie group exponents and SU(2) current algebras, Comm. Math. Phys., 118 (1988), 171-176. 